SUSTAINABLE DESIGN AND SYNTHESIS OF ENERGY SYSTEMS

\author{
A Dissertation \\ Presented to the Faculty of the Graduate School \\ of Cornell University \\ In Partial Fulfillment of the Requirements for the Degree of \\ Doctor of Philosophy
}

\author{
by \\ Jian Gong \\ May 2018
}


(C) 2018 Jian Gong 


\title{
SUSTAINABLE DESIGN AND SYNTHESIS OF ENERGY SYSTEMS
}

\author{
Jian Gong, Ph. D.
}

Cornell University 2018

This dissertation deals with the development of discrete-continuous optimization models and algorithms that address sustainable design and synthesis of energy systems. Specifically, contributions to the following two energy systems are presented, namely an algal biofuel and bioproduct manufacturing system and a shale gas processing and chemical manufacturing system.

The algal biofuel and bioproduct manufacturing system is a promising renewable energy system. In the first related project, we propose a comprehensive superstructure of algal biofuel and bioproducts manufacturing processes and a corresponding mixedinteger fractional programming model to determine the optimal process design with the optimal functional unit based economic and environmental performance. Moreover, we develop a tailored global optimization algorithm to efficiently solve the resulting problem. In the second related project, we propose a two-stage adaptive robust mixed-integer fractional programming model to maximize the return on investment under uncertainty in market related parameters. A tailored optimization algorithm is developed to solve the multi-level optimization problem that cannot be handled directly by any off-the-shelf optimization solvers. In the third related project, we develop a consequential life cycle optimization framework that simultaneously optimizes consequential environmental impacts and economic performance. 
The shale gas processing and chemical manufacturing system is a conventional energy system, but has gained momentum in recent decades due to the successful application of advanced extraction technologies. In the first related project, we develop a general framework for combining product distribution optimization of chemical reactions and superstructure optimization of process flowsheets. A comprehensive superstructure of shale gas processing and chemical manufacturing processes is developed and employed to illustrate the applicability of the proposed framework. In the second related project, we develop a general framework to integrate a novel quantitative measure of resilience and a set of resilience enhancement strategies with process design and operations. The framework identifies a set of disruptive events for a given system, formulates a multiobjective two-stage adaptive robust mixed-integer fractional programming model, and solves the problem with a tailored solution algorithm. The applicability of the proposed framework is illustrated through applications on a chemical process network and a shale gas processing system. 


\section{BIOGRAPHICAL SKETCH}

Jian Gong was born and raised in Xi'an, China. He graduated with a Bachelor's degree in Chemical engineering from Tsinghua University, China in July 2012. In September 2012, he joined Northwestern University to pursue a MS degree. In 2013, he was admitted as a $\mathrm{PhD}$ student and advised by Professor Fengqi You at Northwestern University. In 2016, he joined Cornell University together with Professor You's research group and continued his research on sustainable process design of energy systems. 


\section{ACKNOWLEDGMENTS}

First and foremost, I would like to express my sincerest gratitude to my advisor, Professor Fengqi You. I will never make this $\mathrm{PhD}$ without Professor You's constant encouragement, support, and guidance. He has been always helpful and considerate in the past five years. His broad spectrum of knowledge, insatiable desire for new knowledge, and professionalism are the key to the continuous success of PEESE. His enthusiasm for research are truly inspirational and contagious. I am honored to work with him and learn from his pursuit of excellence, his meticulous attention to details, and his dedication to research, all of which would influence me in the rest of my life. I would like to thank my committee members, Professor Jefferson Tester and Professor Oliver Gao, for their patience and guidance. They have offered very constructive comments and thoughtful feedbacks to make this dissertation possible.

I am grateful for the faculty members in the Robert Frederick Smith School of Chemical and Biomolecular Engineering at Cornell. They offered me the valuable opportunity to graduate in one year with a $\mathrm{PhD}$ degree from the most prestigious university in the world.

My colleagues provided a wonderful experience every day. Dr. Yunfei Chu was instrumental in providing help in modeling and programming. Dr. Dajun Yue set a great example about what we should really strive to achieve in a $\mathrm{PhD}$ program and he was the go-to person when we were confused with anything in the lab. Jiyao Gao, Daniel Garcia, and Karson Leperi helped me a lot by raising critical comments about my presentations and manuscripts and most importantly, they were fantastic friends in leisure time. It was a pleasure to discuss robust optimization with Dr. Chao Shang and Chao Ning and learned from their fruitful works. Visiting scholars including Dr. Chang He, Minbo Yang, Dr. Hua Zhou, and Dr. Na Luo helped me in the research on 
shale gas and we became very close friends. My thanks also go to other visiting scholars and MS students in the group.

Last but not the least, I cannot thank more for the love from my parents. They supported me unconditionally over the last five years. I also want to thank my girlfriend Xian, who has been waiting for me for five years. She was there for me whenever I was in a difficult situation. All of you are of paramount importance in my life. 


\section{TABLE OF CONTENTS}

BIOGRAPHICAL SKETCH............................................................................

ACKNOWLEDGMENTS ................................................................................ vi

TABLE OF CONTENTS ...............................................................................viii

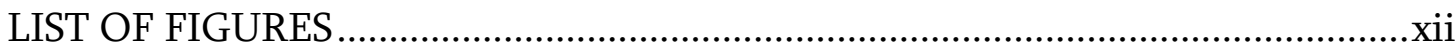

LIST OF TABLES ................................................................................................

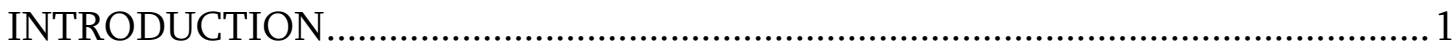

1.1 Superstructure optimization based sustainable process design ......................

1.2 Algal biofuel and bioproduct manufacturing system ……..........................

1.3 Shale gas processing and chemical manufacturing system.......................... 15

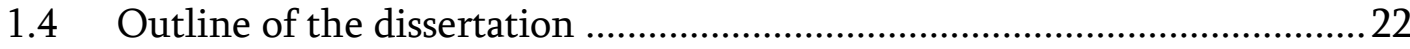

SUPERSTRUCTURE OPTIMIZATION OF ALGAL BIOFUEL AND BIOPRODUCT

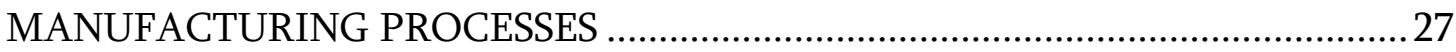

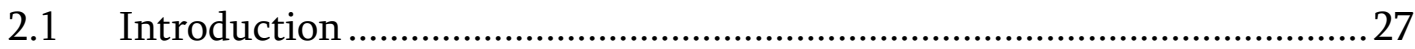

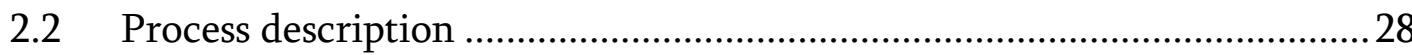

2.3 Life cycle analysis and optimization .......................................................... 47

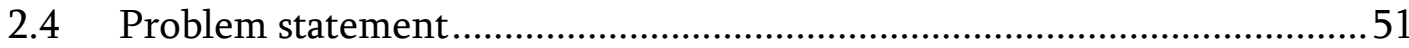

2.5 Model formulation and solution method ……………………................53

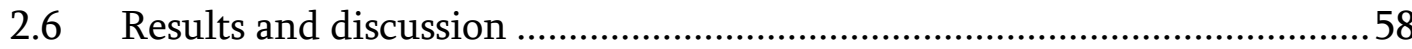

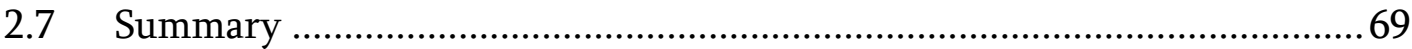

ENERGY SYSTEM DESIGN UNDER UNCERTAINTY............................................71

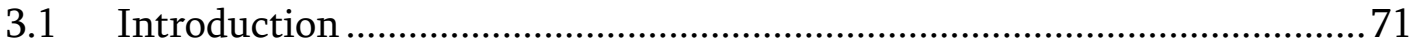

viii 
3.2 Network for producing biofuels and bioproducts from microalgae ..........73

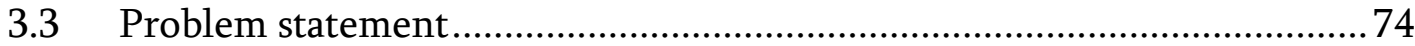

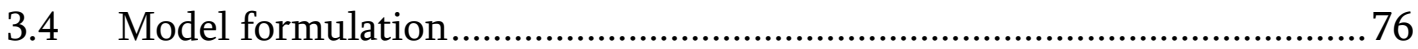

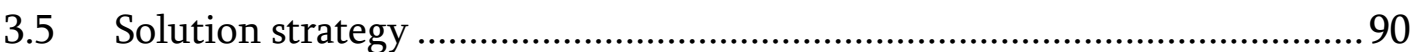

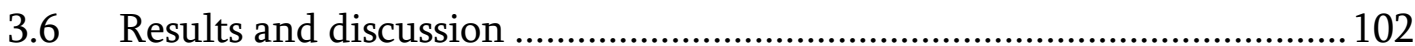

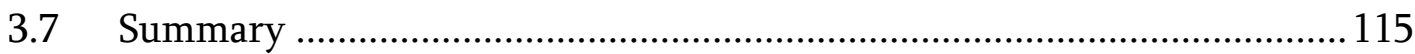

3.8 Appendix A: Superstructure configuration description ......................... 116

3.9 Appendix B: Economic evaluation constraints and definition of ROI ... 119

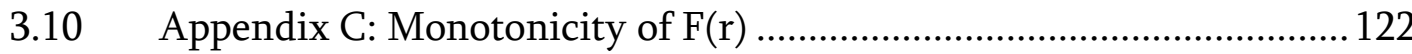

3.11 Appendix D: Subproblem and master problem formulation of

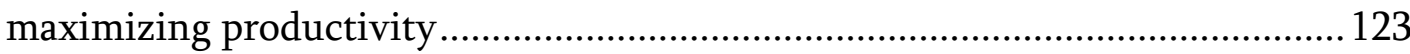

CONSEQUENTIAL LIFE CYCLE OPTIMIZATION …...................................... 125

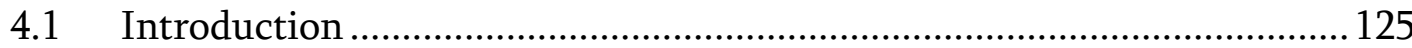

4.2 General conceptual framework..................................................... 127

4.3 Application to algal renewable diesel production ............................... 137

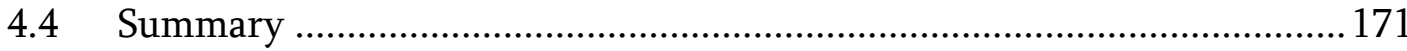

4.5 Appendix A: Model formulation of the consequential LCO problem ..... 173

4.6 Appendix B: Model Formulation of the Auxiliary Parametric Problem .190 SUPERSTRUCTURE OPTIMIZATION COMBINED WITH PRODUCT

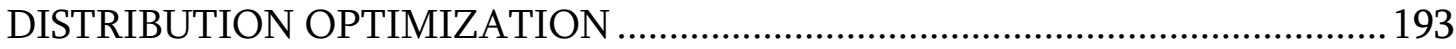

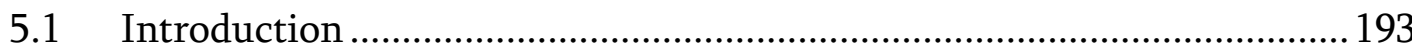


5.2 Problem statement. 196

5.3 A novel modeling framework for process synthesis combining reaction product distribution optimization and superstructure optimization 198

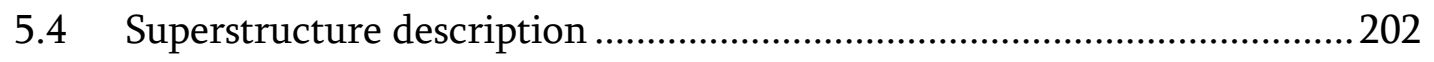

5.5 Product distribution optimization of chemical reactions..........................214

5.6 Superstructure optimization for sustainable process design and synthesis 216

5.7 Application to a Marcellus shale gas feed..................................................223

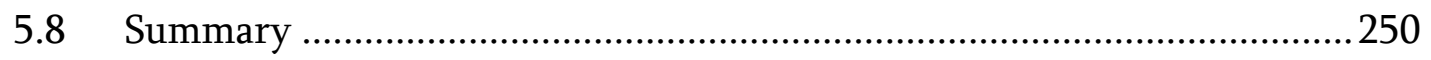

5.9 Appendix A: Model formulation of the product distribution optimization

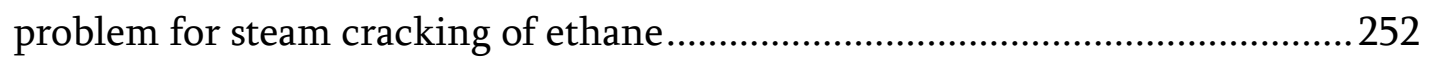

5.10 Appendix B: The optimal results of the product distribution

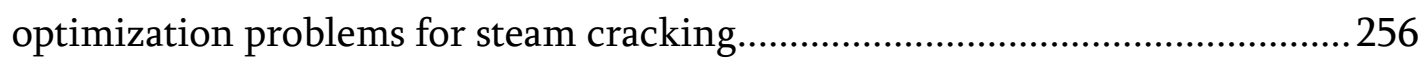

5.11 Appendix C: Model formulation of the superstructure optimization problem 261

5.12 Appendix D: Model formulation of the auxiliary parametric problem 272 RESILIENT DESIGN AND OPERATIONS OF ENERGY SYSTEMS ......................274

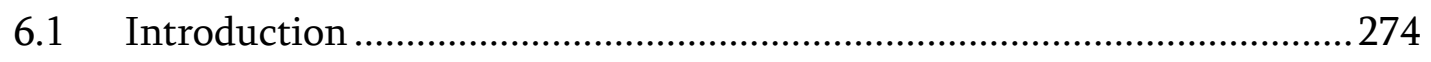

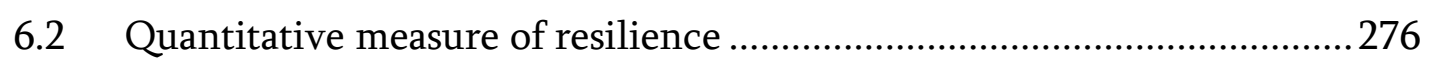

6.3 Resilience enhancement strategies .........................................................282 
6.4 Problem statement for resilience optimization.........................................285

6.5 General framework for resilience optimization......................................287

6.6 Applications of the general framework for resilience optimization.........309

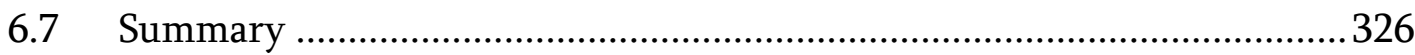

6.8 Appendix A: The conventional design and operations problem................328

6.9 Appendix B: The auxiliary parametric problem ……………………........328

6.10 Appendix C: The master problem of the auxiliary parametric problem 329

6.11 Appendix D: The subproblem of the auxiliary problem 334

6.12 Appendix E: Parameters in the recovery constraints in the applications 337

CONCLUSIONS 339

7.1 Summary of the contributions ……………................................................339

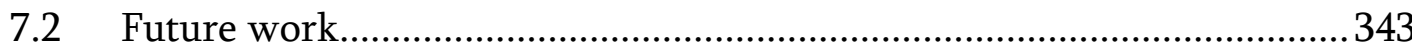

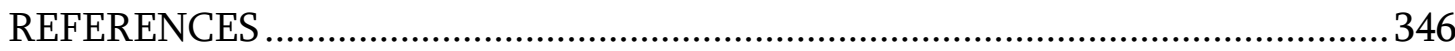




\section{LIST OF FIGURES}

Figure 1. Three major stages for sustainable design and synthesis of energy systems.

Figure 2. The proposed superstructure for the production of bioproducts and biofuel. Blue, purple, and green streams represent system input, system output, and connecting streams, respectively. 30

Figure 3. Process diagram of the cultivation section............................................. 31

Figure 4. Process diagram of the harvesting section. ................................................33

Figure 5. Process diagram of the lipid extraction section. HE represents heat

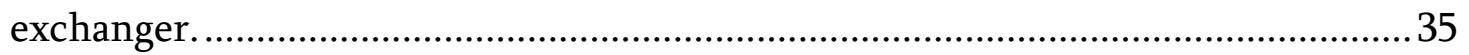

Figure 6. Process diagram of the remnant treatment section.....................................38

Figure 7. Process diagram of the biogas utilization section. ....................................... 38

Figure 8. Process diagram of the biofuel production section..................................... 41

Figure 9. Process diagram of the bioproduct manufacturing section....................... 42

Figure 10. System boundaries of a cradle-to-gate LCA for the production of biodiesel and bioproducts from microalgae include three life cycle stages: feedstock acquisition, transportation (denoted together by blue blocks), and algal biodiesel and bioproduct manufacturing (denoted by green dotted box). Direct and indirect GHG emissions in the manufacturing stage are represented with red and orange 
blocks, respectively. Major process operations and final products are denoted as gray and purple blocks, respectively. P\&T, production and transportation............ 48

Figure 11. Pseudo-code of the global optimization strategy....................................57

Figure 12. Life cycle $\mathrm{CO}_{2}$ equivalent emissions from PG production. ......................61

Figure 13. Total GWP breakdown for the minimum unit GWP of biodiesel. ........63

Figure 14. Pareto-optimal curve associated with the UAC and unit GWP. ............64

Figure 15. Optimal process flow diagram of point 4 ...............................................6

Figure 16. Network for producing biofuels and bioproducts from microalgae. $<1,1>$ open pond; $<1,2>$ flat plate PBR; $<1,3>$ bubble column PBR; $<1,4>$ tubular PBR; $<2,1>$ flocculation with poly-electrolyte; $<2,2>$ flocculation with sodium hydroxide; $<2,3>$ flocculation with poly-aluminum chloride; $<2,4>$ flocculation with aluminum sulfate; $<2,5>$ flocculation with chitosan; $<2,6>$ flocculation with poly- $\gamma$-glutamic acid; $<3,1>$ freeze drying; $<3,2>$ thermal drying; $<3,3>$ pressure filtration; $<3,4>$ centrifugation; $<3,5>$ blank; $<4,1>$ bead beating; $<4,2>$ bead beating; $<4,3>$ microwaving; $<4,4>$ high pressure homogenization; $<4,5>$ sonication; $<4,6>$ blank; $<4,7>$ HTL; $<5,1>$ blank; $<5,2>$ hexane extraction; $<5,3>$ isopropanol/hexane extraction; $\langle 5,4\rangle$ supercritical $\mathrm{CO}_{2}$ extraction; $\langle 5,5\rangle$ hexane extraction; $\langle 5,6\rangle$ butanol extraction; $<5,7>$ supercritical $\mathrm{CO}_{2}$ extraction; $<5,8>$ blank; $<6,1>$ alkaline in-situ transesterification; $<6,2>$ acidic in-situ transesterification; $<6,3>$ enzymatic in-situ transesterification; $<6,4>$ sodium-methoxide-catalyzed transesterification; 
$<6,5\rangle$ heterogeneous acid-catalyzed transesterification; <6.6> supercritical methanol transesterification; $<6,7>$ enzymatic transesterification; $<6,8>\mathrm{Co} / \mathrm{Mo}-$ catalyzed hydroprocessing; <6,9> Ni/Mo-catalyzed hydroprocessing; <6,10> HZSM-5-catalyzed hydroprocessing; $<7,1>$ steam reforming; $<7,2>$ autothermal reforming; $<7,3>$ aqueous-phase reforming; $<7,4>$ propylene glycol production; $<7,5>$ glycerol-tert-butyl ether production; $<7,6>$ PHB production; $<8,1>$ methanol production; $<8,2>$ direct combustion. 73

Figure 17. General flow relationships of technology $j$ in section $i$ in the mass balance. 79

Figure 18. Pseudo code of the proposed algorithm. 100

Figure 19. A heat map for the robust return on investment (\%) under various budgets of uncertainty. DOS is the deterministic optimal solution, and IROS is the illustrative robust optimal solution. 104

Figure 20. Three identified optimal processing pathways. DP is the deterministic optimal pathway; IRP is the illustrative robust optimal pathway. 105 Figure 21. The worst-case uncertainty realizations for the robust optimal solutions with varying budgets of uncertainty. 108

Figure 22. Distribution of the total investment (TI), operating costs (OC), and sales for the deterministic optimal solution (DOS) and the illustrative robust optimal solutions (IROS) 111 
Figure 23. System boundary of attributional LCA (upper) and consequential LCA (lower). 128

Figure 24. Superstructure of the algal renewable diesel production process. 138

Figure 25. Detailed Superstructure of the algal renewable diesel production process. 140

Figure 26. Pseudo code of the tailored global optimization algorithm that integrates the inexact parametric algorithm and the branch-and-refine algorithm. 151

Figure 27. Pareto optimal profiles showing the trade-off between the NPV and the annual GWP. The five pie charts are the cost breakdowns of Solutions A-E. The functional unit based NPV values for Solutions A-E are \$0.4/GJ, \$-29.2/GJ, \$-28.5/GJ, \$-55.5/GJ, and \$0.4/GJ, respectively. The functional unit based GWP values for Solutions $\mathrm{A}-\mathrm{E}$ are $-89,-67,-68,-151$, and $22 \mathrm{~kg} \mathrm{CO}$-eq/GJ, respectively. The renewable diesel production capacities of Solutions A-E are 3.5, 5.0, 10.0, 0.5, and 3.5 million gallons per year, respectively. 153

Figure 28. Optimal process pathway selected by Solution A and Solution E. ....... 156 Figure 29. Annual GWP breakdowns of Solutions A-E in Figure 27. The functional unit based GWP values for Solutions A-E are -89, -67, -68, -151, and $22 \mathrm{~kg} \mathrm{CO}_{2}-$ eq/GJ, respectively. 157

Figure 30. Pareto optimal profiles showing the trade-off between the functional unit based NPV and the functional unit based GWP. The boxes show the major 
process technologies for the optimal solutions with the minimum GWP per GJ of algal renewable diesel. 161

Figure 31. Pareto optimal profiles showing the trade-off between the NPV and the annual ReCiPe endpoint score. The five pie charts show the cost breakdown of Solutions $\mathrm{H}-\mathrm{L}$. The functional unit based NPV values for Solutions $\mathrm{H}-\mathrm{L}$ are \$0.4/GJ, \$-29.2/GJ, \$-28.5/GJ, \$-55.5/GJ, and \$0.4/GJ, respectively. The functional unit based ReCiPe endpoint scores for Solutions H-L are $-9.7,-7.6,-7.6,-4.5$, and 1.7 Pt./GJ, respectively. The renewable diesel production capacities of Solutions H$\mathrm{L}$ are $3.5,5.0,10.0,0.5$, and 3.5 million gallons per year, respectively. 163

Figure 32. Annual ReCiPe endpoint score breakdowns of Solutions H-L in Figure 31. The functional unit based ReCiPe endpoint scores for Solutions H-L are -9.7, $-7.6,-7.6,-4.5$, and $1.7 \mathrm{Pt} . / \mathrm{GJ}$, respectively 165

Figure 33. Environmental profile of Solution H in Figure 31................................ 166

Figure 34. Environmental profile of Solution L in Figure 31. 167

Figure 35. Pareto optimal profiles showing the trade-off between the functional unit based NPV and the functional unit based ReCiPe endpoint score. The boxes show the major process technologies for the optimal solutions with the minimum ReCiPe endpoint score per GJ of algal renewable diesel. 168

Figure 36. Sensitivity analysis for the minimum annual GWP of Solution A in Figure 27. The functional unit based GWP for Solutions $\mathrm{A}$ is $-89 \mathrm{~kg} \mathrm{CO}$-eq/GJ. 169 
Figure 37. Sensitivity analysis for the minimum annual ReCiPe endpoint score of Solution $\mathrm{H}$ in Figure 31. The functional unit based ReCiPe endpoint score for Solutions $\mathrm{H}$ is $-9.7 \mathrm{Pt}$./GJ. 170

Figure 38. Sensitivity analysis for the maximum NPV of Solution A in Figure 27 and Solution $\mathrm{H}$ in Figure 31. The functional unit based NPV for Solution A is $\$ 0.4 / G J$ 171

Figure 39. General flow relationships of technology $j$ in section $i$ in the mass balance $[21,267]$ 174

Figure 40. Flowchart of the proposed process synthesis framework that combines product distribution optimization of chemical reactions and superstructure optimization of the process flowsheet. 198

Figure 41. Overview of the proposed superstructure for an integrated shale gas processing and chemical manufacturing process. 202

Figure 42. Process flowsheet of the acid gas removal, acid gas disposal, dehydration, NGLs recovery, and NGLs separation sections in the superstructure. 205

Figure 43. Process flowsheet of the hydrocarbons conversion, light olefins separation, and C4 separation sections in the superstructure. 206

Figure 44. System boundary of the cradle-to-gate life cycle optimization. The gray arrows denote the material flows; the yellow arrow denotes the utility flow; the 
dark green arrow, the red arrow, and the solid blue arrow denote the wastewater, direct emissions, and direct water consumption from the integrated shale gas processing and chemical manufacturing process, respectively; the dotted orange arrows and the dotted blue arrows represent indirect emissions and indirect water consumption and production, respectively 217

Figure 45. The pseudo code of the tailored global optimization algorithm that integrates the inexact parametric algorithm and the branch-and-refine algorithm.

Figure 46. Optimal process flowsheet corresponding to the minimum GWP per GJ of the products from the shale gas processing part of the integrated shale gas processing and chemical manufacturing process....................................................227 Figure 47. Pareto-optimal curves showing the trade-offs between the NPV per GJ of raw shale gas and the GWP per GJ of raw shale gas. Two good-choice optimal solutions and the selected technologies/processes are also presented. 228 Figure 48. Pareto-optimal curves showing the trade-offs between the NPV per GJ of raw shale gas and the water footprint per GJ of raw shale gas. Two good-choice optimal solutions and the selected technologies/processes are also presented......230 Figure 49. Pareto-optimal surface with the product distributions of steam cracking taken from the literature, showing the trade-offs among the NPV per GJ of raw shale gas, the GWP per GJ of raw shale gas, and the water footprint per GJ of raw 
shale gas. A good-choice optimal solution and the selected technologies/processes are also presented. 233

Figure 50. Pareto-optimal surface with the optimal product distributions of steam cracking, showing the trade-offs among the NPV per GJ of raw shale gas, the GWP per GJ of raw shale gas, and the water footprint per GJ of raw shale gas. A goodchoice optimal solution and the selected technologies/processes are also presented. .234

Figure 51. Optimal process flowsheet of the good-choice optimal solution A in Figure 49. 236

Figure 52. Optimal process flowsheet of the good-choice optimal solution B in Figure 50. 237

Figure 53. Breakdowns of the OPEX and the annualized CAPEX for the goodchoice optimal solution A in Figure 49 and the good-choice optimal solution B in Figure 50. 238

Figure 54. Breakdowns of the GWP and the water footprint of the good-choice optimal solution A in Figure 49 and the good-choice optimal solution B in Figure 50. 242

Figure 55. Comparison of the product flow rates for the optimal solution B based on optimization and the corresponding whole-process simulation. 244 
Figure 56. Sensitivity analysis results for the most economically competitive optimal solution with the product distributions of steam cracking taken from the literature. The high price and the low price correspond to $120 \%$ and $80 \%$ of the current price, respectively. 246

Figure 57. Sensitivity analysis results for the most economically competitive optimal solution with the optimal product distributions of steam cracking. The high price and the low price correspond to $120 \%$ and $80 \%$ of the current price, respectively 246

Figure 58. Upper and lower bounds in each iteration of the global optimization algorithm for solving the superstructure optimization problem in Instance 5 in Table 11 249

Figure 59. Definition of resilience. 278

Figure 60. General framework of resilience optimization. 289

Figure 61. General mass balance model for technology/process alternative $\mathrm{j}$ in section $\mathrm{i}$. 301

Figure 62. The pseudo code of the proposed optimization method that integrates the inexact parametric algorithm and the column-and-constraint generation algorithm. 305

Figure 63. Flowchart of the proposed optimization algorithm. 309

Figure 64. Chemical process network in the first application. 310 
Figure 65. Pareto-optimal curves of the application for chemical process network design and planning. . 312

Figure 66. Heatmap of the optimal resilience results with a capital cost of \$6 MM, various numbers of failed processes, and various tolerance levels. 314

Figure 67. Capital cost breakdowns and worst-case realizations of four optimal solutions. Network design (i) corresponds to the optimal solutions A, B, and D in Figure 65. Network design (ii) corresponds to the optimal solutions C in Figure 65. 316

Figure 68. Technology and process alternatives in the superstructure of shale gas processing and NGLs recovery processes. 318

Figure 69. Process flowsheet of the superstructure for shale gas processing and NGLs recovery processes. 320

Figure 70. Upper and lower bounds of each iteration for the instance of the optimal solution $\mathrm{G}$ 321

Figure 71. Pareto-optimal curve and optimal process design of the superstructure optimization application. 322

Figure 72. Capital cost breakdowns of the optimal solutions E, F, G, and H on the Pareto-optimal curve in Figure 71. 324 
Figure 73. Worst-case realizations of the optimal solutions E, F, G, and H on the Pareto-optimal curve in Figure 71. The selected technology/process alternatives in all the sections are shown in the middle.

325 


\section{LIST OF TABLES}

Table 1. Simulation results for hexane extraction and n-butanol extraction. 35

Table 2. Life cycle $\mathrm{CO}_{2}$ equivalent emissions from hydrogen production. 59

Table 3. Performance of four extreme points. The minimum value in each row is highlighted in bold. 67

Table 4. Computation comparison for four extreme points on the Pareto-optimal curves. 68

Table 5. Major uncertain parameters in the model. 102

Table 6. Key optimal results of the deterministic optimal solution (DOS) and the illustrative robust optimal solution (IROS) 106

Table 7. Computational times (CPUs) for robust optimal solutions with various budgets.

Table 8. Upper and lower bounds of each iteration to obtain the robust optimal solution IROS.

Table 9. Comparison of the GWP per GJ of the products from shale gas processing in this chapter and in the literature. 226

Table 10. Breakdown of CAPEX for optimal solution A and optimal solution B.. 239

Table 11. Computation performance for 6 instances on the Pareto optimal curve with the optimal product distributions of steam cracking using BARON 17.1, SCIP 3.2, and the tailored global optimization algorithm 247 
Table 12. Computational times for the instances of the optimal solutions $\mathrm{A}, \mathrm{B}, \mathrm{C}$, and D.

Table 13. Computational times for the instances of the optimal solutions E, F, G,

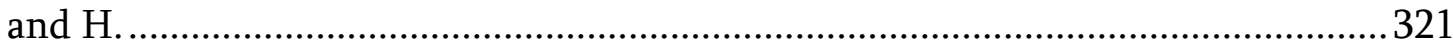




\section{CHAPTER 1}

\section{INTRODUCTION}

Energy systems for energy production and consumption are integral to our modern society $[1,2]$. The energy sector lay the foundation of nearly all other sectors. It is impossible to develop and maintain efficient transportation networks without sufficient fuel supplies. Almost all industries stop operation immediately if they have no access to electricity, which is produced in various energy systems. According to the US Energy Information Administration [3], the total world energy consumption is expected to increase from 575 quadrillion BTU in 2015 to 736 quadrillion BTU in 2040. The additional energy demand will be primarily driven by the strong economic growth of developing countries. Major energy products include fuel products (such as oil, gas, coal, and renewables) and chemicals (such as olefins and aromatics). World's fastest growing energy source is predicted to be renewables, with their consumption to increase $2.3 \%$ each year from 2015 to 2040. Despite the dynamics in the renewable fuel market, fossil fuels will still dominate the world fuel consumption, accounting for $77 \%$ in 2040 . Overall, with a rising energy demand, it becomes more and more significant to increase the efficiency of existing energy systems and develop novel energy systems to help fulfill the energy need. 
The importance of energy systems is multifold. The choices and decisions of energy use directly impacts our living environment. The cost of energy is more than their monetary values, but the economic and environmental effects combined. It is almost a global consensus that the climate-warming trends in the past century are highly likely linked to human activities [4]. The use of energy is usually associated with releasing waste carbon dioxide whether from vehicles or power plants. To reduce carbon footprint, governments around the world set up regulatory policies that encourage the use of cleaner energy and renewables, such as the Renewable Fuel Standard in the US and the Renewable Fuel Directive in the EU [5]. With more and more regulatory documents to support renewable energy products, sustainable energy systems are expected to undergo a rapid expansion in the coming decades $[6,7]$. Other side effects of conventional energy systems, such as toxic pollutions, also threaten the well-being of future generations [8]. As part of the solution to the resulting environmental issues, sustainable energy systems can help satisfy the growing demand of energy products and mitigate the environmental impacts simultaneously by utilizing renewable feedstocks and cutting-edge environmentally friendly technologies [9]. Therefore, there is a need of designing and optimizing sustainable energy systems that address the environmental and economic performance simultaneously so that they outperform 
conventional energy systems. It is the goal of this dissertation to identify and address the research challenges in sustainable design and synthesis of promising energy systems using systematic tools and methods in process systems engineering.

\subsection{Superstructure optimization based sustainable process design}

Sustainability has recently emerged as an important consideration in the design and synthesis of energy systems [10]. Life cycle assessment (LCA) and other methods provide a straightforward way of measuring the sustainability of predefined process designs. Even though the results can be used to compare among various process designs, these static methods become computationally intractable when the most sustainable design must be identified from a large number of design alternatives. 


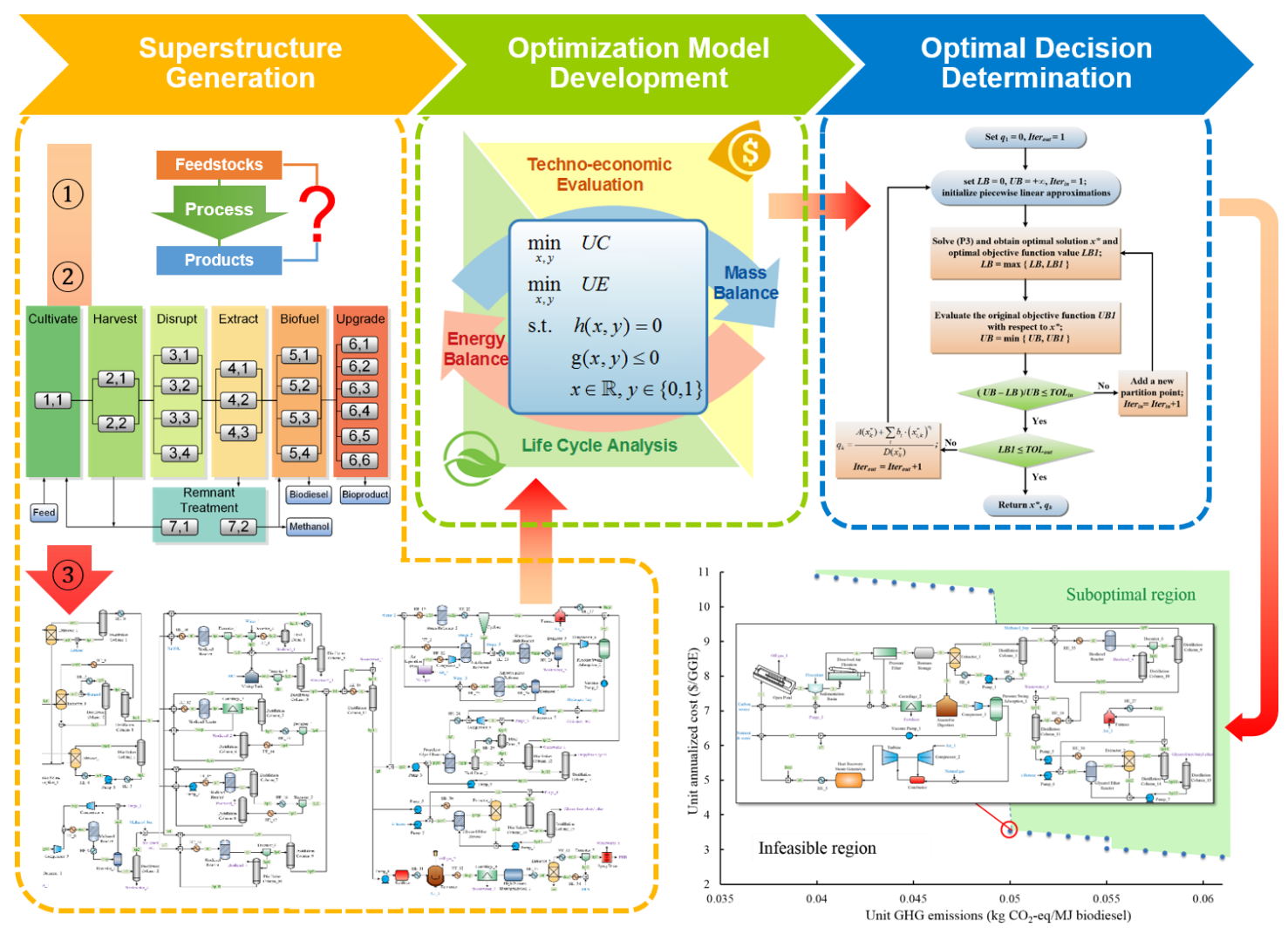

Figure 1. Three major stages for sustainable design and synthesis of energy systems.

Assisted directly by mixed-integer programming techniques, superstructure optimization enables simultaneous evaluation of process/technology alternatives and automatic generation of the globally optimal configuration [11]. A recent systematic method for sustainable design and synthesis of energy systems evolves from superstructure optimization and dynamically integrates the tenets of technoeconomic analysis and LCA into a multi-objective framework [12, 13]. As opposed to other methods, this method enables systematic generation and automatic evaluation of design candidates with the best process economics and highest levels 
of environmental sustainability. Recently this method has been actively applied to various energy systems, including hydrocarbon biorefineries [14-18], algal systems [19-22], polygeneration systems [23-25], shale gas processes [26-28], and network systems [29]. As illustrated in Figure 1, there are three stages in sustainable design and synthesis of energy systems based on superstructure optimization: (1) superstructure generation, (2) optimization model development, and (3) optimal design decision determination [9]. In the dotted orange frame, there are three steps to construct a superstructure: 1) Define design targets; 2) Establish a preliminary superstructure; 3) Specify alternatives. An optimization model is shown in the dotted green frame. The flowchart in the dotted blue frame represents efficient computational algorithms for global optimization. The optimal results are plotted in a Pareto-optimal curve and each point on the curve represents an optimal process design with tradeoffs between the two objective functions. One point representing a "good-choice" design with relatively good performance in both dimensions is shown.

\subsection{Algal biofuel and bioproduct manufacturing system}

The recent years have witnessed the thriving of renewable energy as one of the solutions to climate change and energy dependence [30]. Microalgae and algal 
biorefineries have received increasing attention for producing advanced biofuels and bioproducts. Microalgae refer to a diverse category of microorganisms which can rely on photosynthesis to thrive. Since carbon dioxide is captured and sequestered into biomass during photosynthesis, microalgae are naturally the most efficient contributors to carbon mitigation. Compared with traditional energy crops, microalgae have much higher lipid yields and do not require valuable arable land to cultivate, thus minimizing the competition with crops and the threat to food supplies [31]. Given that lipids are the precursor to biofuels, algal biofuels can be affordable and sustainable if the downstream processing is efficient and reliable. We address three research challenges related to the algal biofuel and bioproduct manufacturing system as detailed in the following subsections.

\subsubsection{Superstructure optimization of algal biofuel and bioproduct manufacturing processes}

Lab-scale designs for emerging advanced biofuel production technologies commonly encounter economic challenges during scale-up, diminishing the commercial viability of an environmentally sustainable process design. In addition to the ongoing research into the productivity of an effective operation, it would be beneficial to produce value-added bioproducts along with biofuels from the 
systematic perspective. For instance, as a result of the continually expanding biodiesel industry, surplus glycerol produced from the transesterification reaction depresses the glycerol price to a very low level. Fortunately, glycerol is a versatile building block chemical, and the conversion of glycerol to its value-added derivatives, rather than selling raw glycerol to the market, offers an opportunity to relieve the increasing environmental pressure and reduce the biodiesel price simultaneously [32-34].

Among a wide array of biomass feedstocks, microalgae have recently received substantial attention in both academia and industry owing to their potential for a high lipid accumulation rate and minimum competition with food and crops $[35$, 36]. There are a few existing publications that offer insights into the sustainable design and synthesis of algal systems. Gebreslassie et al. proposed a detailed superstructure of an algal biorefinery for biofuel production [19]. Despite a large number of technology alternatives included, they focused primarily on the production of algal biofuels, and did not exploit the potential environmental and economic benefits from value-added bioproducts. In order to explore algal biorefinery processes with better economics, Gong et al. optimized the performance of algal biorefinery processes for biological carbon sequestration and utilization [20]. However, the upgrading technology was restricted to 
hydroprocessing for the production of "drop-in" renewable diesel, and did not consider the utilization of byproducts. Martin and Grossmann optimized the coproduction of biodiesel and glycerol ethers from algae oil, but little attention was paid to environmental impacts, and glycerol derivatives were limited to glycerol ethers [37]. Rizwan et al. proposed a superstructure to quickly scan through the algal processing pathways and identify the optimal routes with respect to various objective functions $[38,39]$; however, their discussion did not cover the valueadded bioproducts from glycerol, and no environmental performance was included. An algal biorefinery integrated with a steam electric power plant was reported to be profitable at current biodiesel prices [40], which could be even more affordable if the glycerol was further upgraded. In addition to superstructure optimization, Silva et al. simulated an algae-to-biodiesel process with a biodiesel price of $\$ 4.34 /$ gallon, which is too high to be attractive from the economic perspective [41]. Pokoo-Aikins et al. concluded algal biodiesel is competitive with food-based plant oil [42]. Additionally, much effort was also made on polygeneration systems $[23,25,43]$, thermochemical conversion system [44], and sustainability [14-16, 21, 45], offering useful ideas for the integration of energy systems. Even with the above progress, there still lacks a complete superstructure which not only incorporates existing technologies for the conversion of algal 
biodiesel, but also takes advantage of the versatile glycerol and converts it on site to environmentally sustainable and value-added bioproducts. Process integration is an important method in sustainable process design to explore the possibilities of improving the overall process efficiency by utilizing waste streams and ultimately reduce the overall expenditure and environmental impacts of the entire process. Bioproduct manufacturing production has the potential for boosting the algal biodiesel performance by utilizing byproducts in transesterification and generating carbon dioxide feed for microalgae growth. The aim of the corresponding chapter, therefore, is to explore the potential environmental and economic benefits for the production of both biodiesel and value-added bioproducts from microalgae.

\subsubsection{Handling uncertainty in energy systems}

Return on investment (ROI) is widely used for quantifying investment efficiencies [46]. For algae-based processing pathways, ROI directly reveals their competitiveness and economic viability, which are of great interest to investors and shareholders. Although recent techno-economic analyses have shown relatively high capital costs for popular algae upgrading technologies $[36,47,48]$, the optimal ROI for algae-based process pathways has not been reported in literature. Therefore, there is a practical need to determine the optimal processing 
pathway for producing algal fuels and bioproducts with the highest ROI and identify the technological and economic bottleneck to guide future research [9]. A critical issue pertinent to evaluating the ROI of algae-based processing pathways involves handling uncertainty. Uncertainty can arise almost invariably in network design problems. Many parameters used in existing superstructure optimization models are subject to significant perturbations in reality. However, they are usually treated as deterministic values in existing contributions [19-22, 38, 40-42, 49, 50]. The optimal solutions determined on the nominal values of key parameters frequently lead to poor economic performance when uncertainty is realized [51, 52]. In order to obtain a more realistic economic performance, it is crucial to take uncertainty into consideration explicitly when an optimization model is developed. Two-stage adaptive robust optimization is an emerging method to handle uncertainty in sequential decision-making processes [51, 53-56]. This approach can be used to obtain worst-case optimal solutions that are desired by risk-averse decision makers. Since ROI is defined as the ratio of net earnings over total investment, the objective function in the corresponding optimization problem becomes a fractional term. However, it remains a computational challenge to solve a two-stage adaptive robust optimization problem with a fractional 
objective function. Therefore, one of the goals of this work is to address this challenge.

\subsubsection{Consequential life cycle optimization}

Among a handful of tools for assessing and benchmarking environmental impacts, LCA has received extensive research attention in the past decades by virtue of its unique consideration into the life cycle of a product $[57,58]$. In an LCA study, environmental impacts are usually analyzed and compared among a collection of scenarios or alternatives, and it is tractable to enumerate a small number of scenarios or alternatives for relatively simple systems. However, a comprehensive decision-making process may require analyzing substantially more scenarios. Moreover, if iterative work may be necessary to improve the quality of the assessment results, manually analyzing each alternative system can be overly timeconsuming and labor-intensive. To address this challenge, a life cycle optimization (LCO) methodology was developed $[12,59,60]$. In LCO, the key tenets of LCA are translated into mathematical constraints and integrated within optimization models. By solving the LCO problem, we are able to simultaneously evaluate the environmental and economic performance of alternative systems and automatically identify the one with the optimal performance. LCO provides a systematic tool to 
efficiently evaluate and compare the environmental impacts and performance of other sustainability metrics of a large number of alternative systems.

There are two approaches for conducting an LCA study: attributional and consequential $[57,61]$. So far, all LCO models in the literature followed the attributional LCA approach when evaluating the environmental impacts $[8,9,40$, 62-64]. The attributional LCA approach determines which specific portion of the global environmental impacts can be attributed to a product over its life cycle [57]. Therefore, the system considered in an attributional LCA contains only relevant unit processes that are directly linked to the system under study [65]. By default, the system exerts no influence on the existing markets and is allowed to trade with suppliers and customers with no effect on supply or prices [66]. Therefore, attributional LCA is appropriate when evaluating the environmental impacts of extant systems in the current technosphere. Moreover, the environmental impacts of new production or service systems can be evaluated using attributional LCA only when the scales of such systems are small and markets are not necessarily involved. For example, if the feedstock suppliers save extra products from their previous operations and they agree to sell the feedstocks without going through the markets, the environmental impacts associated with such feedstocks can be evaluated following the attributional approach. However, the "free-to-trade" 
assumption is never completely correct in practice, so the attributional life cycle environmental impacts, which account for contributions beyond process boundaries, are deemed theoretical [67]. If the overlooked environmental consequences can significantly affect the life cycle environmental impacts, the conclusions of attributional LCA studies would be questionable.

Consequential LCA, by contrast, aims to quantify the change in the environmental impacts of a system due to changes in the incorporated processes $[68,69]$. Since the affected processes may not be directly linked to the changed process, additional processes are included in the system of a consequential LCA study. Following the introduction of consequential LCA [70, 71], many methods and applications have been proposed and reported. Perhaps the most widely used method is the scenario analysis method, which derives multiple consequences according to market trends and marginal technologies [72-74]. An alternative method relies on the microeconomic concepts of price elasticity of supply and demand and constructs simple partial equilibrium (PE) models to quantify the indirect impacts $[75,76]$. More sophisticated multi-market multi-region PE models [77], such as the Food and Agricultural Policy Research Institute Models [78], and the U.S. Forest Products Module [79], have been investigated and used. Unlike PE models that are restricted by one or a few economic sectors, computable general equilibrium (CGE) 
models provide more comprehensive consequences in the context of an economy. The CGE models that are employed in consequential LCA include the Global Trade Analysis Project model [80] and the Luxembourg LUXGEM model [81]. Recently, a two-step attributional input-output analysis method has been reported for consequential LCA [82]. Other economic tools that can be incorporated into consequential LCA included rebound effects [83, 84], experience curves [85], industrial cost curves [86], and Rectangular-Choice-of-Technology models [87]. In addition, consequential LCA has been applied to evaluating the life cycle environmental impacts of many products, such as fish [88], dairy [89, 90], soybean meals [72], vegetable oil [74, 91, 92], and biofuels [73, 93-101].

Existing consequential LCA studies follow a sequential evaluation procedure, i.e. building a life cycle inventory before environmental impacts can be assessed. If the product under study is derived from a complex process system, the overall performance of the system would rely on a set of highly coupled decisions for process design and synthesis, such as the selection of technologies/processes in different sections of the system and the temperatures and pressures in the reactors of these technologies/processes. The combination of these decisions can result in an infinite number of possible system conditions that may not be well-represented by only a few scenarios. In such a circumstance, it is necessary to identify the 
optimal decisions following a systematic LCO methodology $[12,59,60]$. However, the prevalent attributional LCO framework fails to account for environmental consequences through the lens of market mechanisms. As a result, the attributional LCO framework is not appropriate for consequential LCO modeling. To the best of our knowledge, there is no consequential LCO framework reported in the literature.

\subsection{Shale gas processing and chemical manufacturing system}

In recent years, shale gas is regarded as an important driver leading the changes in North America's energy landscape [102, 103]. Due to the successful application of advanced extraction technologies, total natural gas production in the U.S. is predicted to increase by $45 \%$ by 2040 [104]. As a result of the boom in upstream shale gas production, there is a need for additional facilities to absorb the burgeoning supplies of shale gas [105]. Shale gas processing systems removes valuable natural gas liquids (NGL) and undesired constituents from raw shale gas [106] and serve as important components in the shale gas value chain [107]. The resulting NGL products can be further utilized to manufacture value-added chemical products. The rapid expansion of the shale gas industry will expedite the deployment of more shale gas processing and chemical production systems in the 
near future. We address two research challenges in the shale gas processing and chemical manufacturing system as detailed in the following subsections.

\subsubsection{Superstructure optimization of shale gas processing and chemical manufacturing processes}

An integrated shale gas processing and chemical manufacturing process simultaneously produces pipeline quality gas and a collection of value-added chemicals from wellhead shale gas. The value-added chemicals can bring a higher profit margin than the NGL products from a conventional shale gas processing system, contributing to a better economic performance under relatively low energy prices. By sharing assorted utilities, process integration could lead to a higher energy efficiency, mitigating the environmental impacts associated with utility generation systems. To fully exploit these advantages, it is important to address the optimal process design of the integrated shale gas processing and chemical manufacturing process that should be both economically competitive and environmentally sustainable.

Shale gas has gained increasing research attention in the recent years. As a versatile and cost-effective material from shale gas, methane was used as the feedstocks of methanol and syngas production processes $[63,108]$. A raw shale gas feed can 
consist of up to $30 \%$ NGLs by volume depending on the specific geological locations [109]. Given the relatively high market prices of NGLs-based chemicals, several process designs were proposed to integrate shale gas processing and olefin production systems. Differences among these process designs lie in the types of olefin products $[26,27,110]$, and the scales of the systems [111]. Additionally, optimization models for shale gas supply chain were proposed [107, 112-115]. Recent works also address uncertainties in shale gas feed compositions and estimated ultimate recovery $[116,117]$. Although several simplified product distribution models for certain well-studied reactions were integrated into superstructure optimization models [118], the product distributions of most chemical reactions were considered as fixed parameters. However, the product distribution of a chemical reaction can vary drastically under different operating conditions. As markets change rather dynamically, the most lucrative product portfolio decades ago may become less economically attractive or even unprofitable today. To identify the optimal performance of a process that involves chemical reactions, the product distributions of the chemical reactions should be optimized to enhance the yields of more profitable products in a superstructure optimization based process synthesis framework. To the best of our knowledge, 
such a holistic systems modeling and optimization framework for process synthesis has not been proposed in the existing literature.

There are several research challenges towards a holistic process synthesis framework that combines product distribution optimization of chemical reactions and superstructure optimization of the process flowsheet. The first one is how to account for reaction product distributions in the superstructure optimization framework. Product distributions of chemical reactions are constrained by the continuity equations for reaction flows, which are further governed by complex reaction kinetics. Additionally, product distributions of chemical reactions can influence the operating conditions of other unit operations in the process. These factors regarding product distributions of chemical reactions must be considered systematically in a holistic framework for process synthesis. The second challenge is the need of high-fidelity process data that are tailored to the product distributions of the involved chemical reactions in the superstructure. As these important process data are typically not available in the literature, it is necessary to develop simulation models for all the involved processes. However, due to the complexity of each process, careful tuning must be performed extensively to ensure the feasibility of the corresponding simulation model and satisfy the specifications of a collection of products. Given that many technology/process 
alternatives are considered in the superstructure, performing rigorous simulations for all the processes with distinct operating conditions can be extremely laborintensive and effort-taking. The third challenge is how to efficiently solve the resulting superstructure optimization problem. To capture all levels of details, the superstructure optimization problem is formulated as a large-scale mixed-integer nonlinear programming (MINLP) problem that can be computationally intractable for general-purpose global optimization solvers. Therefore, a tailored global solution algorithm needs to be developed to circumvent the computational challenge. The goal of the corresponding chapter is to address these challenges.

\subsubsection{Resilient design and operation of energy systems}

A major goal of risk management is to avoid the occurrences of undesired events by implementing effective prevention and protection strategies [119]. However, many disruptive events, such as Hurricane Sandy in 2012 and the Haiti and Chile Earthquake in 2010, suggest that not all unexpected events can be avoided [120]. Disruptive events usually strike a process system and cause critical failures in vulnerable processes [121]. The undesired consequences of disruptive events

highlight the need for enhancing the resilience of process systems [122-126]. System-level resilience was first introduced in ecology as the persistence of systems 
regarding unexpected change and disturbance [127]. For infrastructure systems, resilience was described as the ability to mitigate hazards and minimize the reduction in the quality of life [128-133]. In process control, a resilient plant could move fast and smoothly from one operating condition to another and dealt effectively with disturbances [134]. Although resilience may be interpreted by different terminologies in various contexts [135], a resilient system is always capable of absorbing a portion of the impacts from disruptive events and recovering to the original state rapidly.

There are several mathematical programming models in the literature for resilient design and operations. A group of studies focused on system operations after the occurrence of disruptive events and optimized the efficiency of recovery scheduling [136-138]. Others developed holistic optimization models for resilient design and operations simultaneously. Since the availability of each process after the occurrence of disruptive events was uncertain, systematic approaches for handling uncertainty, such as stochastic programming [139] and robust optimization [53, 140-142], were employed for resilient design and operations of infrastructure networks and supply chains [143-147]. However, resilience in these optimization models was not quantified explicitly as an intrinsic property of the system. Moreover, as these models considered infrastructure systems with only one 
species, they were not suitable for chemical process design problems that involved multiple species in each stream. A relevant concept of resilience was flexibility [148] and a quantitative measure was developed to predict the probability of feasible operation for a design [149]. Another relevant concept of resilience was reliability, which described the failure rate of a unit/process over time due to equipment aging $[150,151]$. Therefore, the strategies for improving reliability, such as employing parallel production units, could also be considered for enhancing the resilience of the overall process systems [152, 153].

There are several research challenges to develop a general framework for resilience optimization. The first challenge is to propose a novel quantitative measure of resilience for process systems. The quantitative measure should be able to account for both performance degradation and system recovery. Additionally, resilience is an intrinsic property of a system, indicating that the quantitative measure should be independent of external systems or volatile markets. The second challenge is how to model resilience enhancement strategies, and how to integrate the resilience enhancement strategies and process models into an integrated systems analysis and optimization framework. Because the resilience of a process system is relevant to both safety and operability, the system performance under the worstcase realization of disruptive events is of paramount importance [154]. Adaptive 
robust optimization is an emerging method to handle uncertainty in sequential decision-making processes and to hedge against the worst-case realization of uncertainty $[155,156]$. Although adaptive robust optimization could be an effective tool for addressing problems on resilient design and operations, there are research challenges on developing a two-stage adaptive robust optimization model for resilience optimization and on effective solution of the resulting optimization problem that cannot be tackled directly by any off-the-shelf optimization solvers due to its multilevel structure. The goal of the corresponding chapter is to develop a resilient design and operation framework for energy systems

\subsection{Outline of the dissertation}

This dissertation addresses the sustainable design and synthesis of two energy systems, namely the algal biofuel and bioproduct manufacturing system (Chapters $2-4)$ and the shale gas processing and chemical manufacturing system (Chapter 5 and Chapter 6). The roadmap of the dissertation is provided as follows.

In Chapter 2, we address the sustainable design and synthesis of algal biofuel and bioproduct manufacturing processes. We propose by far the most comprehensive superstructure capable of producing biodiesel, hydrogen, propylene glycol, glycerol-tert-butyl ether, and poly-3-hydroxybutyrate from microalgae. The major 
processing sections include cultivation, harvesting, lipid extraction, remnant treatment, biogas utilization, biofuel production, and bioproduct manufacturing. Based on the superstructure, we integrate a cradle-to-gate LCA and technoeconomic analysis with multiobjective optimization to simultaneously optimize the environmental and economic performance. We also apply a tailored global optimization algorithm to efficiently solve the problem in reasonable computation times.

In Chapter 3, we address the optimal design of a large-scale processing network for producing a variety of algae-based fuels and value-added bioproducts under uncertainty. We develop by far the most comprehensive processing network with 46,704 alternative processing pathways. Based on the superstructure, a two-stage adaptive robust mixed-integer fractional programming model is proposed to tackle the uncertainty and select the robust optimal processing pathway with the highest return on investment. Since the proposed problem cannot be solved directly by any off-the-shelf solver, we develop an efficient tailored solution method that integrates a parametric algorithm with a column-and-constraint generation algorithm.

In Chapter 4, we develop a consequential LCO framework that simultaneously optimizes consequential environmental impacts and economic performance. We 
propose a general system boundary that encloses processes linked by markets. Based on the general system boundary, we develop a multiobjective optimization model, which integrates process models and market models with the tenets of consequential LCA and techno-economic analysis methodologies. To efficiently solve the resulting nonconvex MINLP problem, a global optimization algorithm is proposed to integrate the inexact parametric algorithm and the branch-and-refine algorithm. The application of the proposed framework is illustrated through a case study of producing renewable diesel from microalgae. We conduct detailed market analysis to identify the consequences associated with the renewable diesel production process.

In Chapter 5, we propose a novel process synthesis framework that combines product distribution optimization of chemical reactions and superstructure optimization of the process flowsheet. A superstructure with a set of technology/process alternatives is first developed. Next, the product distributions of the involved chemical reactions are optimized to maximize the profits of the effluent products. Extensive process simulations are then performed to collect high-fidelity process data tailored to the optimal product distributions. Based on the simulation results, a superstructure optimization model is formulated as an MINLP to determine the optimal process design. A tailored global optimization 
algorithm is employed to efficiently solve the large-scale nonconvex MINLP problem. The resulting optimal process design is further validated by a wholeprocess simulation. The proposed framework is applied to a comprehensive superstructure of an integrated shale gas processing and chemical manufacturing process, which involves steam cracking of ethane, propane, $n$-butane, and $i$ butane.

In Chapter 6, we propose a general framework for resilience optimization is proposed that incorporates an improved quantitative measure of resilience and a comprehensive set of resilience enhancement strategies for process design and operations. The proposed framework identifies a set of disruptive events for a given system, and then formulates a multiobjective two-stage adaptive robust mixedinteger fractional programming model to optimize the resilience and economic objectives simultaneously. The model accounts for network configuration, equipment capacities, and capital costs in the first stage, and the number of available processes and operating levels in each time period in the second stage. A tailored solution algorithm is developed to tackle the computational challenge of the resulting multi-level optimization problem. The applicability of the proposed framework is illustrated through applications on a chemical process network and a shale gas processing system. 
The dissertation concludes in Chapter 7. 


\section{CHAPTER 2}

SUPERSTRUCTURE OPTIMIZATION OF ALGAL BIOFUEL AND BIOPRODUCT MANUFACTURING PROCESSES

\subsection{Introduction}

In this chapter, we develop by far the most comprehensive superstructure, which is able to produce biodiesel and four types of bioproducts, including hydrogen, propylene glycol (PG), glycerol-tert-butyl ether (GE), and poly-3-hydroxybutyrate (PHB). In particular, the hydrogen can be produced by either steam reforming, autothermal reforming, or aqueous-phase reforming. The superstructure also includes a methanol synthesis process in the biogas utilization section. Additionally, we simulate the lipid extraction processes using hexane and nbutanol as extractants for data validation. Based on the proposed superstructure, a cradle-to-gate life cycle assessment (LCA) is performed to account for the life cycle greenhouse gas (GHG) emissions associated with three life cycle stages, namely feedstock acquisition, transportation, and algal biodiesel and bioproduct manufacturing. Following a life cycle optimization methodology, we further integrate the LCA and techno-economic analysis with multiobjective optimization to simultaneously optimize the environmental and economic performance. A tailored global optimization algorithm is applied to circumvent the computational 
difficulties caused by separable concave terms and mixed integer fractional terms in the objective functions. The results from this study justify the significance of manufacturing bioproducts in algal biodiesel processes and offer guidance to related technology advancement.

The rest of this paper is organized as follows. The process description is presented in the next section, followed by a brief explanation of a cradle-to-gate LCA. Necessary information of the optimization problem is provided in the problem statement section. Later, we propose a multiobjective mixed-integer nonlinear programming (MINLP) model and the global optimization strategy for efficient computation. In the next section, we discuss the LCA results, economic performance, and the computational comparison. The article is concluded in the last section.

\subsection{Process description}

A process superstructure refers to a collection of process alternatives that are candidates for a feasible or optimal design [157]. In this chapter, we propose by far the most comprehensive superstructure for the production of biodiesel and bioproducts from the microalgae strain Chlorella vulgaris, as illustrated in Figure 2. The superstructure can be divided into seven sections: cultivation, harvesting, lipid 
extraction, remnant treatment, biogas utilization, biofuel production, and bioproduct manufacturing. The details of each section of the superstructure are given in the following subsections. 


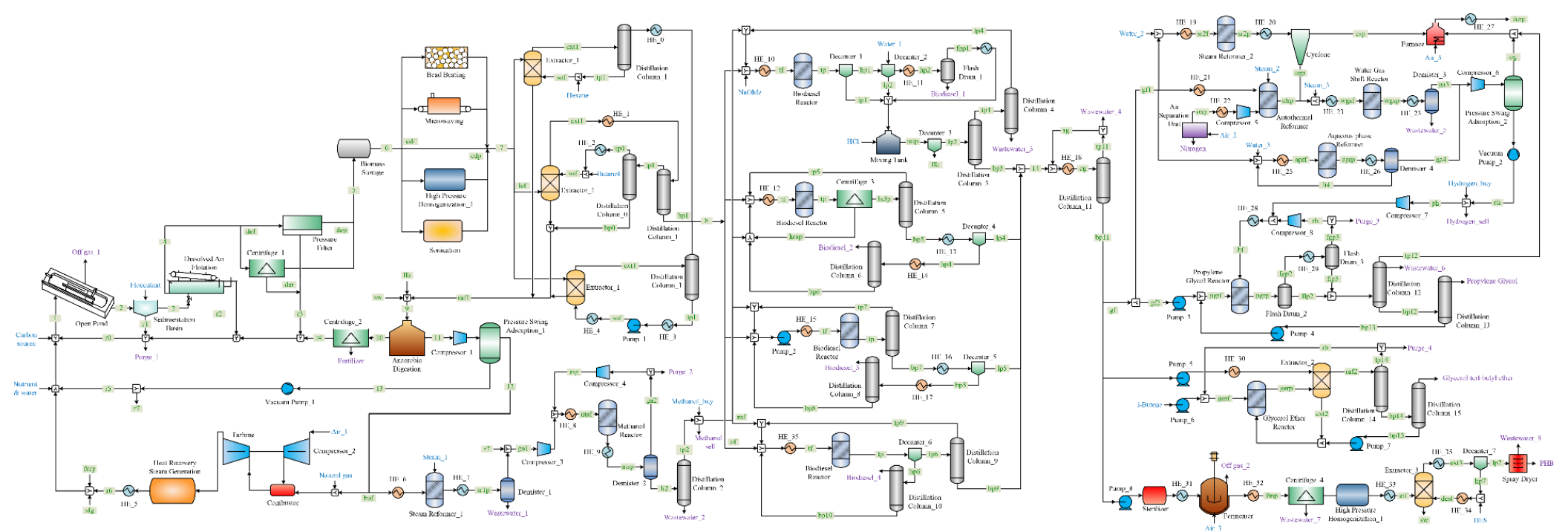

Figure 2. The proposed superstructure for the production of bioproducts and biofuel. Blue, purple, and green streams represent system input, system output, and connecting streams, respectively. 


\subsubsection{Cultivation}

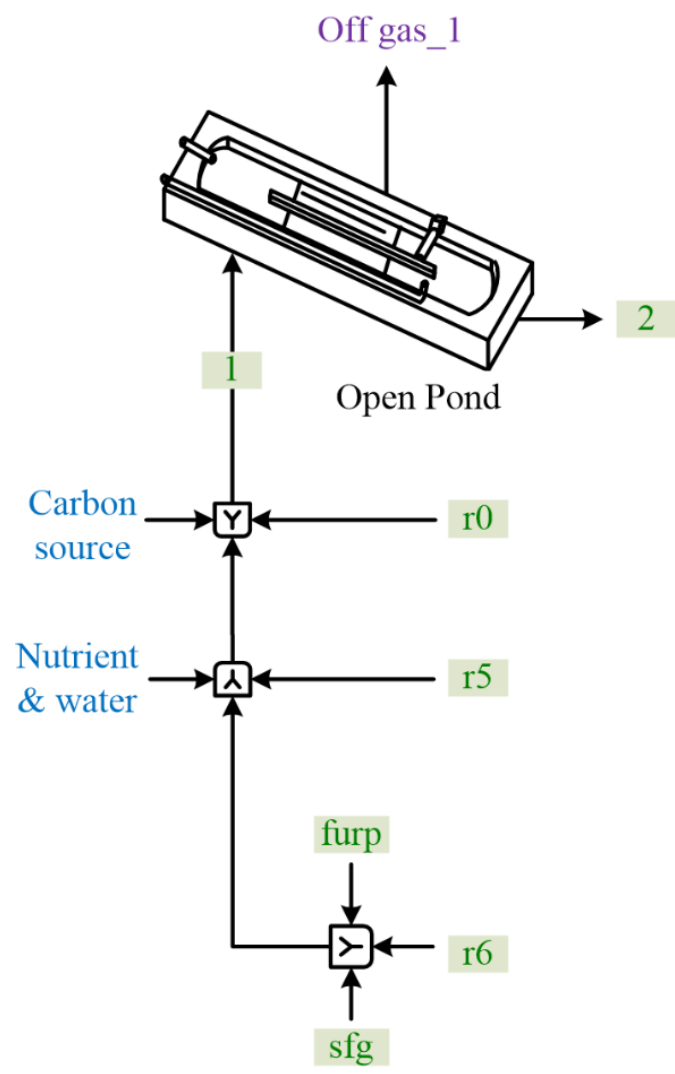

Figure 3. Process diagram of the cultivation section.

Given a carbon source, nutrients, and water, a commonly cultivated microalgae strain, Chlorella vulgaris (nitrogen starvation may significantly increase the lipid content, but a widely used lipid content of $25 \mathrm{wt} \%$ is applied), is flourished in raceway open ponds (Figure 3). The carbon dioxide concentration in the carbon source is $21.14 \%$, which falls in the range of healthy growth of microalgae [158]. Algal biodiesel and bioproducts are considered environmentally beneficial because waste carbon dioxide generated in the process can utilized by microalgae as extra 
carbon source. Enlightened by this idea, we recycle the off-gas streams to the open ponds instead of releasing them to the environment. 1 kilogram of carbon dioxide consumed contributes to 0.526 kilograms of microalgae [159]. However, it is impossible for microalgae to capture and digest the entire input carbon source in a single-pass injection. Due to the difficulty of collecting emitted gas from the open ponds, as much as $25 \%$ of the carbon dioxide together with the oxygen produced during photosynthesis is expelled to the environment [159].

\subsubsection{Harvesting}

Containing only $0.015 \mathrm{wt} \%$ of mature biomass, the immediate product from the open ponds is unable to be handled and utilized by downstream technologies. Therefore, as shown in Figure 4, we employ a three-step strategy in the harvesting section to reduce the undesired water content. The first equipment unit is a sedimentation basin, which separates most of the surplus water from the dilute product via autoflocculation and achieves a concentration of $1 \mathrm{wt} \%$ [160]. The flocculant added to assist gravitational separation is a type of cationic polyelectrolyte and the effective concentration is $3 \times 10^{-6}$ according to existing experimental results [161]. We assume the impact of the flocculant on the downstream processing is negligible due to the small quantity. The second step involves the use of a dissolved air flotation system, which increases the algal 
concentration to $10 \mathrm{wt} \%$ [47]. Finally, the algae slurry is processed by one of the two technology alternatives: pressure filtration and centrifugation. Both technologies can thicken the algae slurry to $30 \mathrm{wt} \%[162,163]$, but pressure filtration consumes less energy while a centrifuge is less expensive to install. A total of $5 \mathrm{wt} \%$ of the separated water is purged, while the remaining is recycled to the open ponds. As discussed in our previous work, the equipment in the cultivation and harvesting sections operate only during daytime because sun light is one of the necessary ingredients for the algae growth. Therefore, a biomass storage tank to store $50 \%$ of biomass is employed at the end of the harvesting section so that the downstream processes receive biomass feed continuously throughout the day. Inoculation is not considered in this superstructure.

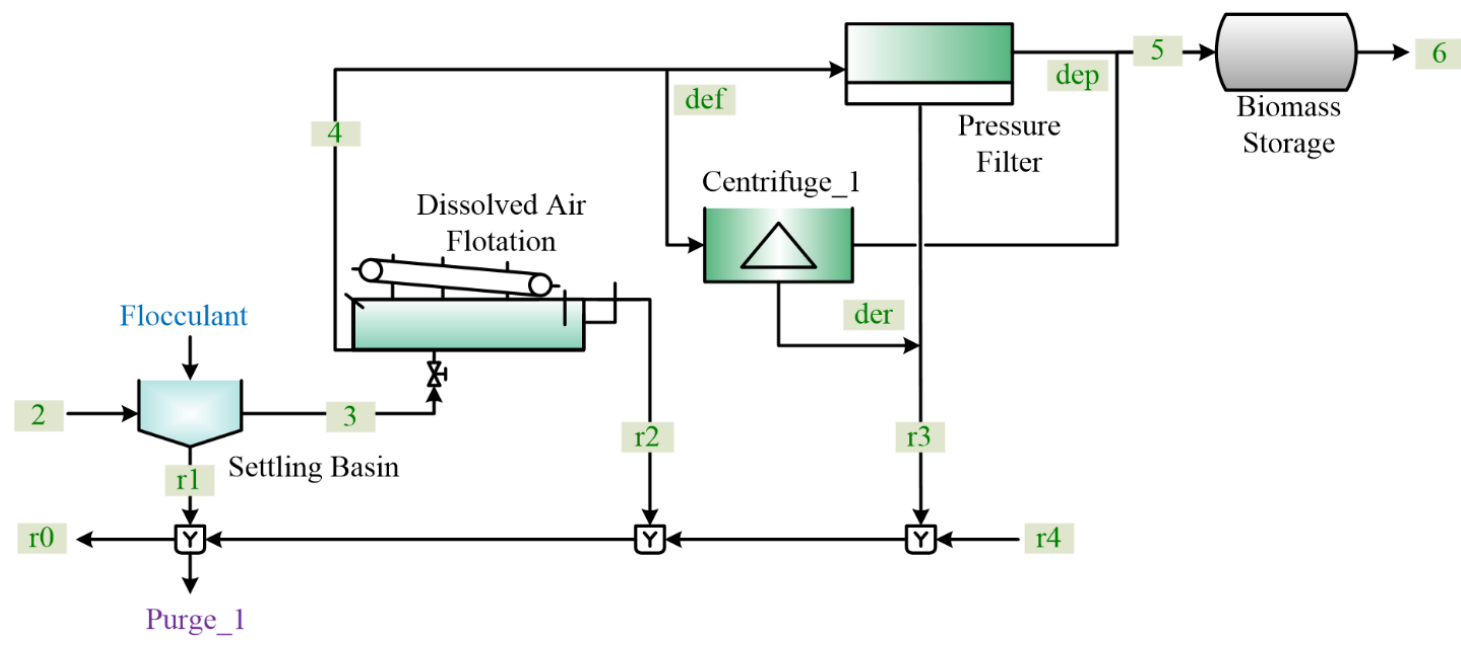

Figure 4. Process diagram of the harvesting section. 


\subsubsection{Lipid extraction}

Since lipid materials, in the form of fatty acids and triacylglycerols, are mixed in the algal cells with other components, such as proteins and carbohydrates, solvent extraction is applied to separate these convertible lipids. Before sending the algal biomass directly to extractors, it should be noted that the inclusion materials are protected by cell membranes and cell walls, which may to some extent inhibit the effective contact between the extractant and the target lipids. Following the results from Lee [164], we consider five cell disruption methods in the superstructure to improve the efficacy of the solvent extraction system. In Figure 5, bead beating, microwaving, high pressure homogenization, and sonication are four energyintensive cell disruption methods with various disruption effects. The last option is to bypass the cell disruption, which lowers the lipid content available for extraction, but avoids the exceeding energy consumption. The mass balance model of this section is shown in equations (S20)-(S39) and corresponding data are listed in Table S19 and Table S20. 


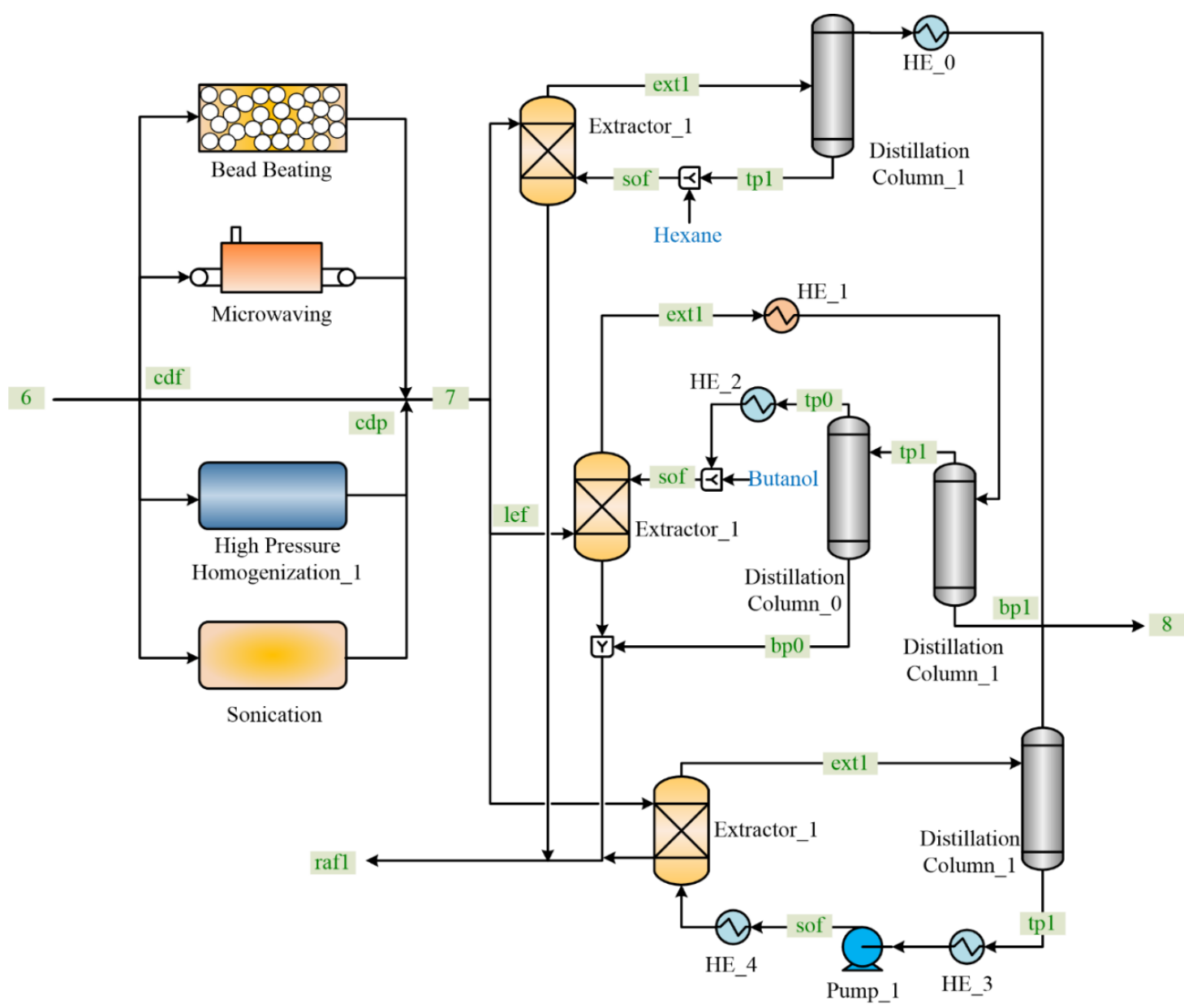

Figure 5. Process diagram of the lipid extraction section. HE represents heat exchanger.

Table 1. Simulation results for hexane extraction and n-butanol extraction.

\begin{tabular}{ccccc}
\hline & & mass flow rate of lipid & 43.75 & $\mathrm{~kg} / \mathrm{h}$ \\
mass flow rate of water & 0 & $\mathrm{~kg} / \mathrm{h}$ \\
Hexane extraction & Stream ext 1 & $\begin{array}{c}\text { mass flow rate of } \\
\text { solvent }\end{array}$ & 1,000 & $\mathrm{~kg} / \mathrm{h}$ \\
\cline { 2 - 5 } & Distillation & top temperature & 345 & $\mathrm{~K}$ \\
& column & bottom temperature & 420 & $\mathrm{~K}$ \\
DC1 & heat duty & 0.2 & $\mathrm{MW}$
\end{tabular}




\begin{tabular}{|c|c|c|c|c|}
\hline & & $\begin{array}{l}\text { cold duty } \\
\text { capital cost }\end{array}$ & $\begin{array}{l}0.19 \\
0.37\end{array}$ & $\begin{array}{c}\text { MW } \\
\$ M \\
\text { M }\end{array}$ \\
\hline \multirow{3}{*}{$\begin{array}{l}\text { n-butanol } \\
\text { extraction }\end{array}$} & Stream ext 1 & $\begin{array}{l}\text { mass flow rate of lipid } \\
\text { mass flow rate of water } \\
\text { mass flow rate of } \\
\text { solvent }\end{array}$ & $\begin{array}{r}6,684 \\
5,044 \\
24,160\end{array}$ & $\begin{array}{l}\mathrm{kg} / \mathrm{h} \\
\mathrm{kg} / \mathrm{h} \\
\mathrm{kg} / \mathrm{h}\end{array}$ \\
\hline & $\begin{array}{c}\text { Distillation } \\
\text { column } \\
D C 1\end{array}$ & $\begin{array}{c}\text { top temperature } \\
\text { bottom temperature } \\
\text { heat duty } \\
\text { cold duty } \\
\text { capital cost }\end{array}$ & $\begin{array}{c}380 \\
459 \\
17.23 \\
15.63 \\
0.77\end{array}$ & $\begin{array}{c}\mathrm{K} \\
\mathrm{K} \\
\mathrm{MW} \\
\mathrm{MW} \\
\$ \mathrm{M} \\
\mathrm{M}\end{array}$ \\
\hline & $\begin{array}{c}\text { Distillation } \\
\text { column } \\
D C O\end{array}$ & $\begin{array}{l}\text { top temperature } \\
\text { bottom temperature } \\
\text { heat duty } \\
\text { cold duty } \\
\text { capital cost }\end{array}$ & $\begin{array}{c}383 \\
403 \\
44.29 \\
43.84 \\
1.42 \\
\end{array}$ & $\begin{array}{c}\mathrm{K} \\
\mathrm{K} \\
\mathrm{MW} \\
\mathrm{MW} \\
\$ \mathrm{M} \\
\mathrm{M}\end{array}$ \\
\hline
\end{tabular}

The extraction technologies belong to wet extraction, which avoids the intensive energy consumption in drying the microalgae to powers. Most existing research regarding lipid extraction is limited to finding appropriate solvents at the laboratory scale [165], but these experimental results might be less attractive and profitable when they are embedded in a complete process. For instance, butanol is a commonly available solvent with a good lipid extraction yield and little side effect to most downstream processes, but the butanol recovery step consumes a large amount of utilities due to the existence of water. In this chapter, we consider using solvent extraction to separate lipids from wet algal paste without completely 
drying the biomass. Three chemicals, namely hexane, n-butanol, and supercritical carbon dioxide, are considered as alternative solvents in the lipid extraction. Because of a lack of justified data of complete extraction-recovery cycles for hexane and n-butanol, the processes for these solvents are constructed and simulated using Aspen Plus [166].

Hexane is not miscible in water, so only one distillation column is necessary to partition lipid and the solvent. In contrast, butanol is soluble in water and at least two distillation columns are needed if the solvent is to be reused. As a result of the large amount of energy spent on separating water and alcohol, lipid extraction by butanol loses its significant advantage in a complete extraction-solvent recovery system. The cursory analyses are concluded from the simulation results as listed in Table 1. The data for supercritical carbon dioxide extraction is retrieved from literature $[167,168]$.

\subsubsection{Remnant treatment}

Despite the significance of algal lipids in biofuel conversion, they only contribute to as much as $25 \mathrm{wt} \%$ of the dry biomass. In fact, a substantial proportion of the energy captured by algae still remain in the lipid-extracted algal cells, or algal remnant. In the proposed superstructure (detailed by Figure 6), algal remnant is introduced into digesters, where long chain organic molecules are decomposed 
into short chain carbon dioxide and methane by active anaerobic bacteria. Methane is carefully purified from the waste carbon dioxide through a pressureswing-adsorption system. Simultaneously, important elements like nitrogen and phosphorus are released to the effluent and reused in algae cultivation after solid fertilizers are separated by centrifugation.

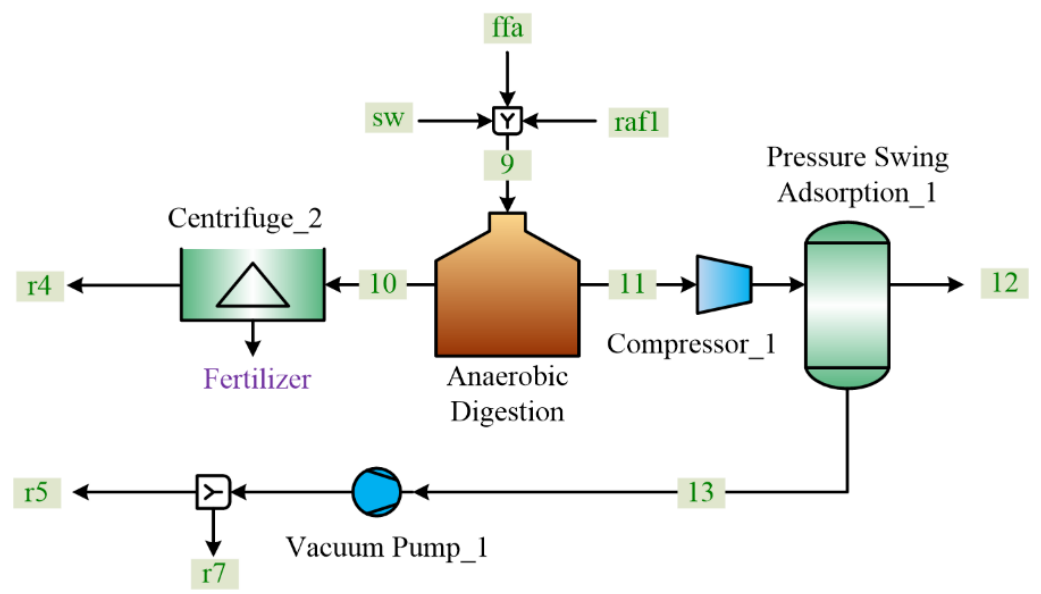

Figure 6. Process diagram of the remnant treatment section.

\subsubsection{Biogas utilization}

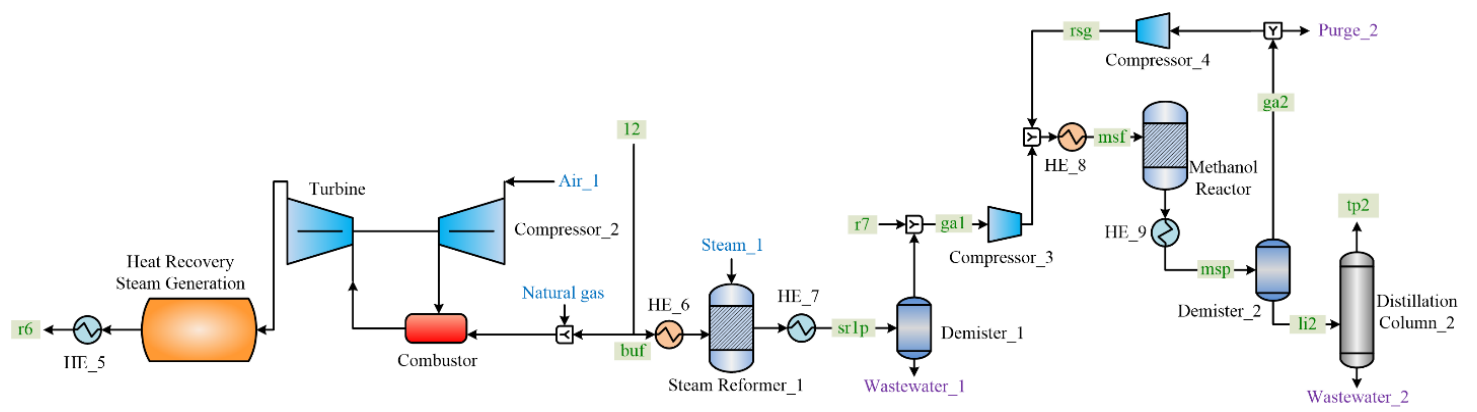

Figure 7. Process diagram of the biogas utilization section.

In order to fully exploit the methane from anaerobic digestion, two methods are considered: on-site power generation and methanol synthesis. If power generation 
is chosen (left in Figure 7), the fuel gas is sent to a combustor, and the corresponding flue gas enters a turbine and a heat recovery steam generation system sequentially, resulting in an overall energy-to-electricity conversion of 58

$\%$ on the basis of lower heating values of the combustible gases [159]. Otherwise, if methanol synthesis is selected, the methane feed is introduced into a reformer together with steam, and converted into carbon monoxide and hydrogen. Dried by a demister, the syngas product is enriched by a carbon dioxide stream and converted to methanol. Finally, methanol, waste water, and unreacted gases are obtained from two distillation columns. A small proportion of the unreacted gases are purged to a furnace, while the remaining are recycled to the methanol reactor. In case that the methanol is more than the downstream demand or no methanol is produced in this section, we allow methanol to be sold to or purchased from the market. The possibility of buying and selling methanol simultaneously is eliminated by employing the binary variable.

\subsubsection{Biofuel production}

A lot of studies have focused on transesterification of lipids from biomass resources and various reaction conditions have been tested and simulated $[169,170]$. In Figure 8 , four transesterification technologies are considered, namely, sodium methoxide-catalyzed transesterification [171], heterogeneously catalyzed 
transesterification [172], enzyme-catalyzed transesterification [173], and supercritical methanol transesterification [172]. Whatever technology selected, the core reaction involves transforming one molecule of triglyceride to one molecule of glycerol and three molecules of fatty acid alkyl esters, or biodiesel. Although methanol is commonly regarded as the other reactant in transesterification, short chain alcohol chemicals, like ethanol, propanol, and butanol are able to complete the same reaction. Therefore, we assume that butanol residue from the lipid extraction section undergoes transesterification with priority. Once the butanol runs out, or no butanol is mixed in the lipid feed, methanol is introduced and continues the reaction until the lipids run out. 


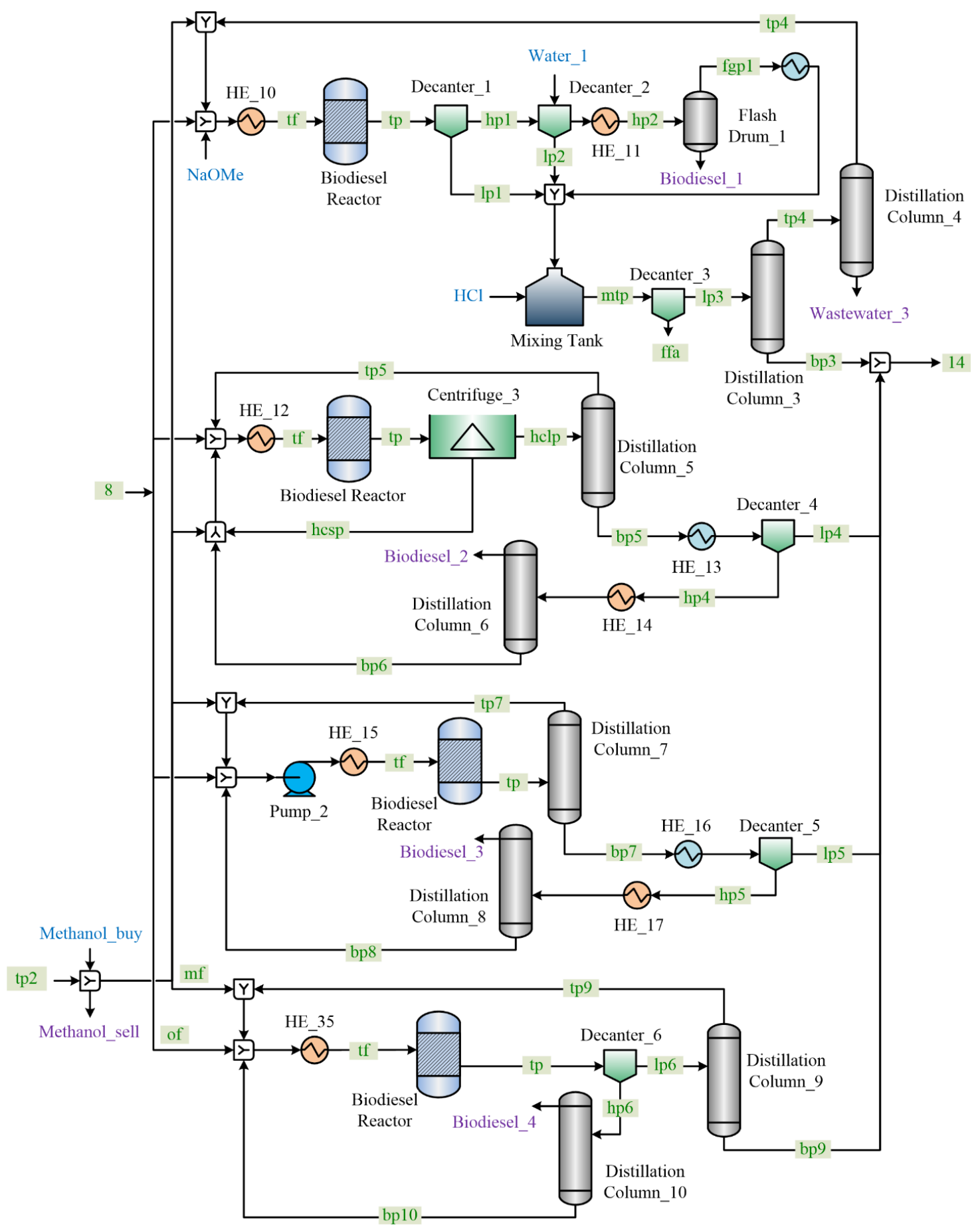

Figure 8. Process diagram of the biofuel production section. 


\subsubsection{Bioproducts manufacturing}

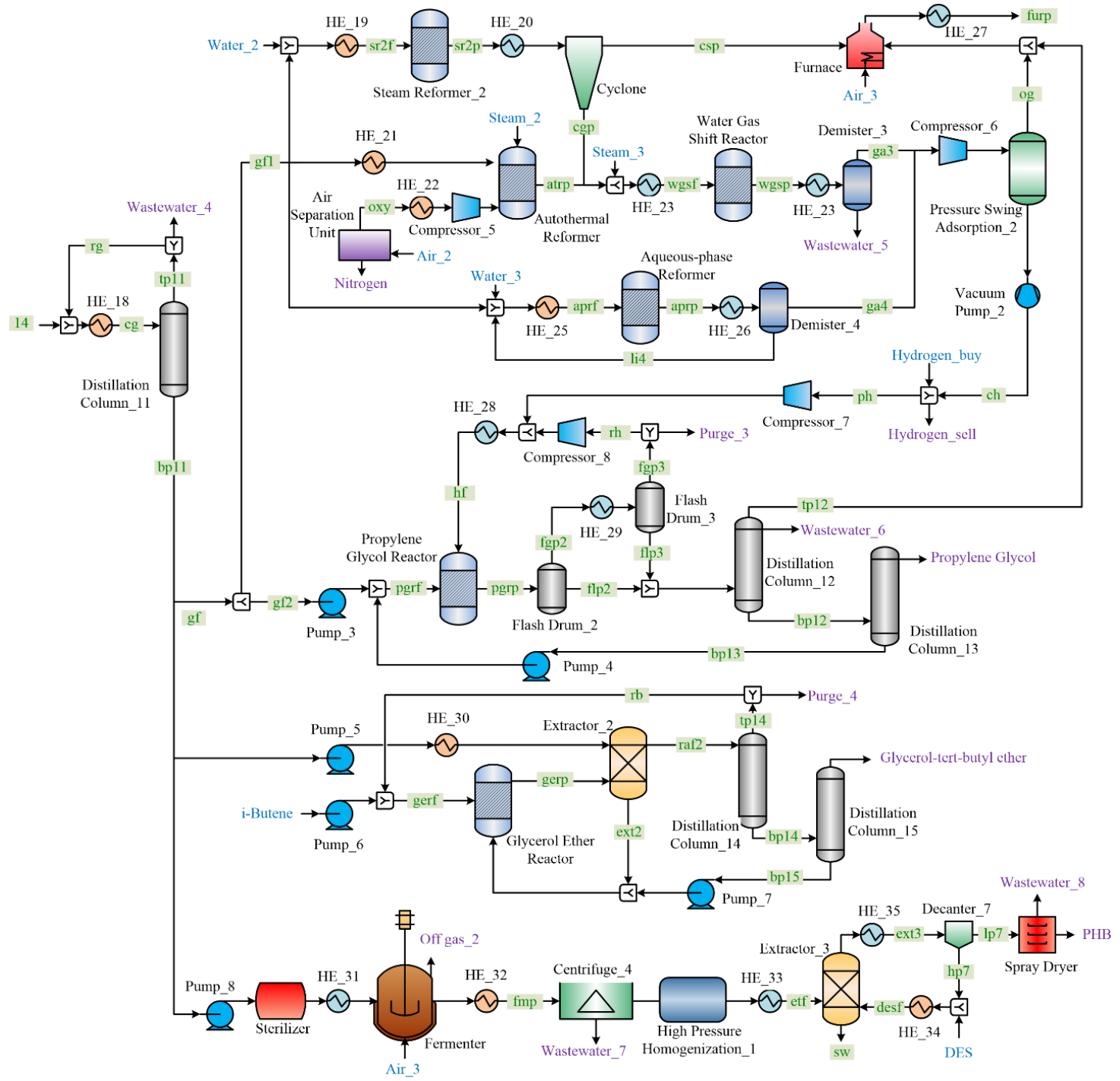

Figure 9. Process diagram of the bioproduct manufacturing section.

The immediate products from the biofuel production section are biodiesel and glycerol with an approximate weight ratio of 10:1. Glycerol is an important platform chemical which can be upgraded to value-added chemicals through selective oxidation, etherification, hydrogenolysis, dehydration, reforming, 
carbonate, epichlorohydrin, etc. [33] In this chapter, we consider the conversion of purified glycerol from microalgae to four types of bioproducts, including hydrogen, PG, GE, and PHB. The process flow diagram of this section is shown in Figure 9.

\subsubsection{Hydrogen}

Yielding only water during oxidation, hydrogen is regarded as a clean and efficient fossil fuel substitute [174]. Furthermore, it participates in some important industrial reactions, like hydrotreating and hydrocracking [175]. Motivated by these merits, researchers attempted to produce hydrogen from various feedstocks, including surplus glycerol from biodiesel production, and through a plethora of technologies, including gasification, pyrolysis, catalytic steam reforming, and so forth $[174,176]$. In this chapter, we investigate hydrogen production from purified glycerol via three different methods: steam reforming, autothermal reforming, and aqueous-phase reforming.

In the first method, purified glycerol and water are sent to a steam reformer at 565 ${ }^{\circ} \mathrm{C}$ and converted to carbon monoxide, hydrogen, methane, ethylene and char $[177,178]$. The feed glycerol is heated to $565^{\circ} \mathrm{C}$ by a heat exchanger based on the values reported by Jones et al. [175] As an endothermic reaction, glycerol steam reforming extracts heat from the furnace, where all the purged gases are burned. The solid char is eliminated from the reaction products via a cyclone, while the 
remaining gases and a stream of steam are injected into a water-gas-shift reactor, where most of the carbon monoxide and water are converted to carbon dioxide and hydrogen [179]. After surplus water is separated by a demister, the gas products undergo pressure swing adsorption to obtain a hydrogen stream with 99 wt $\%$ purity. As also assumed by Jones et al. [175], the $1 \%$ impurities primarily consist of carbon dioxide which does not influence the propylene glycol production.

Hydrogen production via autothermal reforming of glycerol is able to achieve a net zero energy consumption by appropriately combining the endothermic reforming reaction and the exothermic oxidation of glycerol [180]. Regarding the oxidation reaction, the consumed oxygen is produced on site by an air separation unit, whose by-product, nitrogen, can be sold to the market [181]. Next, gas products are sent to a water-gas-shift reactor, a demister, and a pressure-swing-adsorption system sequentially in a similar fashion to steam reforming.

The last hydrogen production technology is aqueous-phase reforming. As reported by Guo et al. [182], this reforming reaction takes place under a relatively mild temperature of $225^{\circ} \mathrm{C}$ in the presence of a Ni-B amorphous alloy catalyst, but achieves a single loop conversion of only $9 \%$. Large amounts of unreacted glycerol and water are recycled from a demister to the inlet of the reactor. Since the 
hydrogen selectivity is already high in aqueous-phase reforming, the gas products bypass the energy intensive water-gas-shift reaction for hydrogen enrichment, and undergo a pressure-swing-adsorption system for hydrogen purification directly after the demister.

\subsubsection{Propylene glycol}

PG, or 1,2-propanediol, commonly produced by propylene oxidation, serves as an important feedstock of unsaturated polyester resins [183]. As opposed to the conventional route, $\mathrm{PG}$ is produced through catalytic hydrogenolysis of glycerol in the present superstructure. Glycerol and hydrogen react at $190{ }^{\circ} \mathrm{C}$ and become a mixture of water, PG, propanol, acetone, and unreacted glycerol and hydrogen. Two flash drums are employed sequentially to separate unreacted hydrogen from the other products, and $5 \%$ of the gas materials are purged to the furnace $[184$, 185]. Next, $99.5 \mathrm{wt} \%$ PG product is achieved by using two distillation columns to separate waste and unreacted materials from the product.

\subsubsection{Glycerol-tert-butyl ether}

Another important group of glycerol derivatives are GE's. Specifically, di-tertbutyl glycerol ether (DE) and tri-tert-butyl glycerol ether (TE) are very useful additives to diesel fuels due to their antidetonant and octane-enhancing nature [183]. Additionally, they can reduce the emissions of fumes, particulates, and 
carbon oxides resulting from fuel combustion. In this chapter, a mixture of DE and TE can be produced following the purification of crude glycerol.

Besides glycerol, the other important raw material for GE production is isobutylene, which is mixed with $10 \mathrm{wt} \%$ isobutane. The etherification reaction takes place at $90{ }^{\circ} \mathrm{C}$ and $1.4 \mathrm{MPa}$ [186]. Both the feed and the products maintain their liquid states under such conditions. Before being sent to the reactor, the purified glycerol is regarded as an extractant to separate most of the unreacted glycerol and mono-tert-butyl glycerol ether from the etherification reaction. Next, the raffinate is introduced into a distillation column where unreacted isobutylene and isobutane are separated from the top of the column and recycled to the reactor after a small amount is purged. The remaining products undergo distillation again and become qualified products.

\subsubsection{Poly-3-hydroxybutyrate}

As a group of polyhydroxyalkanoates, PHB's are attractive biodegradable substitutes for conventional petroleum-derived plastics and can be upgraded to fibers, films, and heteropolymers [187]. According to Posada et al. [188], PHBs can be synthesized by Cupriavidus necator JMP 134 over a glycerol-based substrate. In the PHB process, the sterilized glycerol is sent into a fermentation reactor with abundant air. The fermentation reaction converts glycerol to water, carbon 
dioxide, intracellular PHB, and other cell building materials, which are regarded as remnant. Next, a centrifuge is employed to reduce the water content in the fermentation products. After cell disruption by high pressure homogenization, the concentrated biomass is sent to an extractor so that the PHB product can be extracted by diethyl-succinate (DES). The raffinate is sent to anaerobic digestion for energy recovery, while the extracts are introduced to a decanter after being cooled to $25^{\circ} \mathrm{C}$ by a heat exchanger. The PHB product in the light phase is further dried by a spray drier, and DES solvent in the heavy phase is reused.

\subsection{Life cycle analysis and optimization}

The goal of this study is to quantitatively determine the minimum life cycle environmental impacts of the bioproducts and biodiesel from microalgae. The functional unit of this LCA is defined as $1 \mathrm{~kg}$ of bioproduct manufactured, following the definitions by Rostkowski et al. [189] In this chapter, the impact category to assess the life cycle environmental performance of manufacturing bioproducts and biodiesel from microalgae is dedicated to global warming. The corresponding environmental metric, GWP, is calculated using the IPCC (International Panel on Climate Change) method with characterization factors of a 100-year horizon (GWP 100a) [190]. 


\subsubsection{System boundaries}

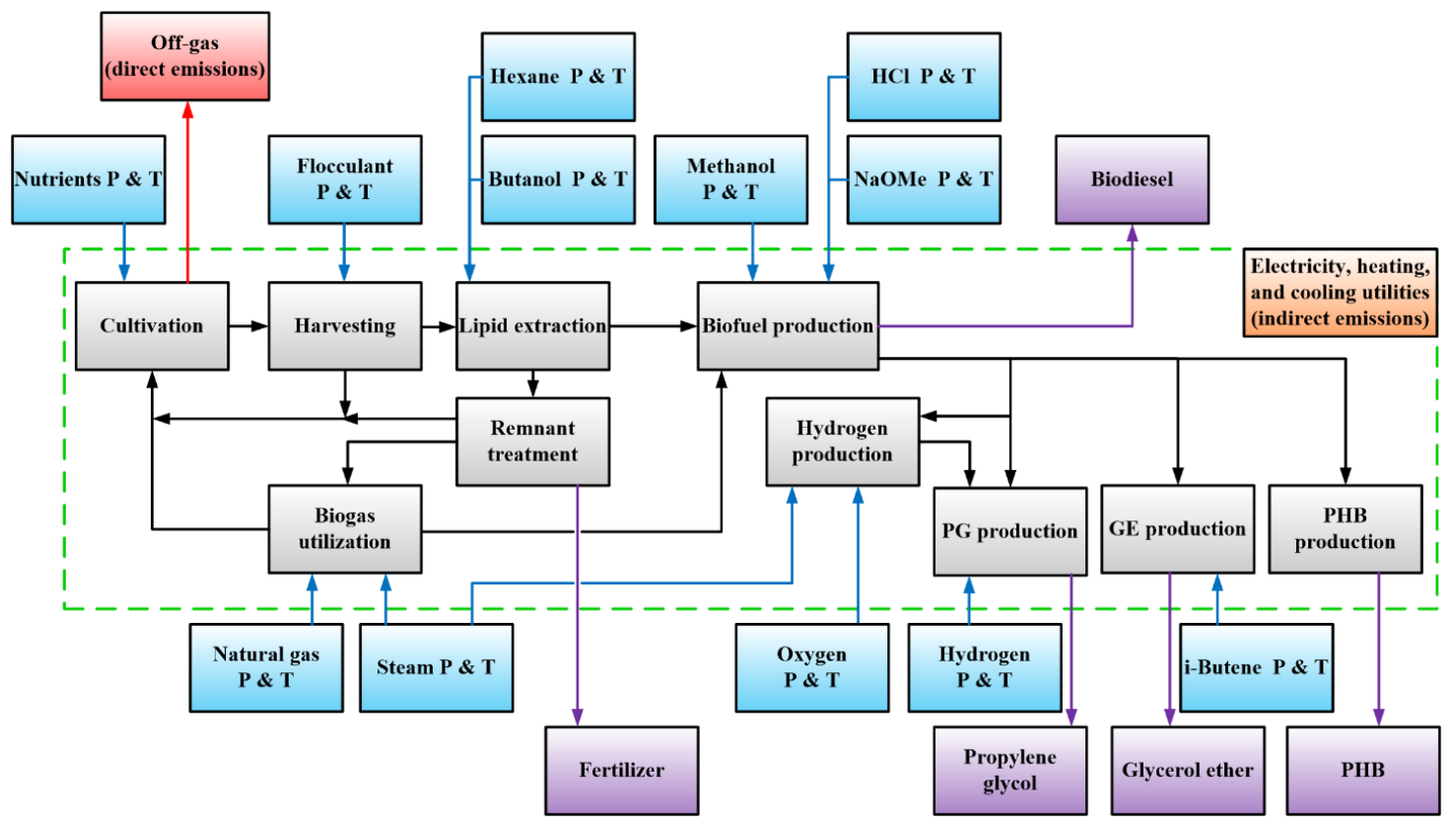

Figure 10. System boundaries of a cradle-to-gate LCA for the production of biodiesel and

bioproducts from microalgae include three life cycle stages: feedstock acquisition,

transportation (denoted together by blue blocks), and algal biodiesel and bioproduct manufacturing (denoted by green dotted box). Direct and indirect GHG emissions in the manufacturing stage are represented with red and orange blocks, respectively. Major process operations and final products are denoted as gray and purple blocks, respectively. P\&T, production and transportation.

Refined from the proposed superstructure, the system boundaries for the LCA is illustrated with major processing blocks, emissions, and input and output materials in Figure 10. As a process-based LCA, the system boundaries take into consideration the life cycle GHG emissions associated with three life cycle stages, namely feedstock acquisition, transportation, and algal biodiesel and bioproduct manufacturing. The direct emissions in the algal biodiesel and bioproduct manufacturing stage include off-gas released in the cultivation section and 
wastewater throughout the entire process. The indirect emissions account for life cycle GHG emissions associated with electricity, heating, and cooling utilities consumed in the process. Due to a lack of information about the end-of-life phases of several non-fuel chemicals, the LCA is confined to a "cradle-to-gate" analysis. We also assume that the carbon dioxide source comes from a nearby coal-fired power plant and water is also available in nearby sources. As a result, the environmental impacts associated with flue gas acquisition and transportation, as well as water transportation, are excluded from this analysis [191, 192]. Furthermore, life cycle GHG emissions associated with materials and energy consumed in construction of the processes are also excluded [193].

\subsubsection{Data source}

The LCA in this chapter is based on mass and energy balances of the comprehensive superstructure. We make our attempts to find and organize the most up-to-date data available in the literature. Data selection is prioritized to U.S. databases, such as electricity prices and average transportation distances. Process data related to mass and energy balances, as well as parameters for economic evaluation are extracted from literature and Aspen Plus simulation results [166]. The distances for delivering various feedstocks to the algal biorefinery are assumed to be the average U.S. transportation values specified by commodity categories 
[194], while the life cycle impact assessment parameters for translating the mass of various materials, energy consumption, and transportation quantity (measured by the product of distance and mass of the feedstock) to corresponding GWP contributions are taken from the Ecoinvent database [195].

\subsubsection{Co-product allocation}

There are several potential co-products other than bioproducts (hydrogen, PG, GE, and PHB) and biodiesel in the superstructure, namely fertilizer from anaerobic digestion, methanol and electricity from biogas utilization, and nitrogen and hydrogen from hydrogen production. According to the ISO guidelines [196], we treat the surplus electricity as an environmental credit and subtract the corresponding environmental impact from the total GWP. Next, a portion of the electricity-modified total GWP is allocated to each co-product based on its economic value when leaving the system boundaries.

\subsubsection{Life cycle optimization}

Some metrics are developed to quantitatively evaluate a process. For instance, the environmental impact can be quantified by the total GWP, while the economic performance of a process can be evaluated by the total annualized cost (TAC) [46]. Commonly, life-cycle and techno-economic analyses require pre-defined processes and systems, therefore lacking the ability to generate feasible process designs from 
a process superstructure with better or best process performance (e.g. economics and environmental sustainability). In this chapter, an integrated methodology termed life cycle optimization [12], which enables the multiobjective optimization of a system under both economic and environmental criteria, is used to simultaneously optimize environmental and economic performance of manufacturing bioproducts and biodiesel from microalgae. This methodology requires establishing a rigorous equation-based multiobjective mathematical optimization model based on the proposed superstructure for manufacturing algal bioproducts and biodiesel. The problem statement, model formulation, and optimization algorithms are introduced in the following sections.

\subsection{Problem statement}

In this life cycle optimization problem, the most comprehensive superstructure by far is developed for the production of algal bioproducts and biodiesel. The superstructure consists of seven sections and a variety of technology alternatives. Specifically, the cultivation section is established with raceway open ponds. The harvesting section encompasses a settling basin, a dissolved air flotation system, and either a pressure filter or a centrifuge in the end. The lipid extraction section is comprised of a biomass storage tank, a cell disruption system with five technology alternatives, and three solvent extraction options. The remnant treatment section 
is made up of an anaerobic digester and a centrifuge, followed by a biogas utilization section where biogas is consumed either by combustion or methanol synthesis. The biofuel production section employs four transesterification technology alternatives to produce biodiesel, and the last bioproduct manufacturing section could synthesize hydrogen with three technologies, PG, GE, and PHB.

The mass flow rate of the feed carbon source is fixed. Physical properties of feed and product materials are given, including, but not limited to, species distributions of nutrients, flocculant, natural gas, steam, hexane, n-butanol, methanol, sodium methoxide, hydrochloric acid, oxygen, hydrogen, isobutylene, and DES. Additionally, we are given product distributions and conversions of microalgae cultivation, anaerobic digestion, combustion of organic materials, steam reforming reactions of biogas and glycerol, transesterification, water-gas-shift reaction, and synthesis reactions for methanol, PG, GE, and PHB. Operating temperatures and pressures of all operations as well as split fractions of separation units are known. Unit power, heating, or cooling utility consumption, or temperature differences and efficiency of heat-exchanging equipment are available in the literature. We are also given parameters pertaining to economic evaluation including life span of the biorefinery, interest rate, sizing factors, base-case costs and mass flow rates, 
chemical engineering plant cost indices, capital-to-investment parameter, and prices of feed materials and products. For the purpose of the LCA, transportation distances and damage factors for GWP calculations are known.

The aim of this optimization model is to determine the most sustainable strategy of producing value-added bioproducts and biodiesel. Decision variables of this multiobjective optimization problem include technology selections in harvesting, lipid extraction, biogas utilization, biofuel production, and bioproduct manufacturing, mass flow rates of all streams, power, heating, or cooling utility consumption or generation of different equipment, equipment capital cost, operating cost, and GWP's for feedstock acquisition, transportation, and algal biodiesel and bioproducts manufacturing.

\subsection{Model formulation and solution method}

A bi-criteria MINLP model is formulated to optimize the design and operation of algal biodiesel and bioproducts manufacturing processes. This model consists of an environmental objective function, an economic objective function, and four types of constraints. In the mass balance constraints, the relationship between the input and output streams of a separation unit is developed based on the split fraction and mass conservation of every species (44 species are considered). The reactions are described with stoichiometric coefficients and conversions, or product 
distributions, depending on the type of data available in the literature. Also, integer variables are introduced to model technology selection decisions. The energy balance of each unit is closely related to the corresponding mass balance such that the electricity, heating, and cooling utility consumption are based on the mass flow rates of the input and output materials. The economic evaluation constraints calculate annualized investment cost based on equipment capital costs which follow a nonlinear scaling rule. These constraints also determine the annual operating cost, taken as the sum of feedstock cost, utility cost, operating \& maintenance cost, and waste treatment cost. Finally, the TAC is calculated as the sum of the annualized investment cost and the annual operating cost. The constraints for life cycle environmental impact analysis first evaluate the life cycle GHG emissions originating from raw material acquisition, transportation, and algal biodiesel and bioproduct manufacturing. Next, the emissions are translated into environmental impacts, which are finally aggregated to the total GWP.

The bi-criteria MINLP model consists of one environmental objective function and one economic objective function. Each objective function could optimize either total quantities or unit quantities. For instance, problem (P1) minimizes total GWP and TAC simultaneously. The constraints can be found in the published article [22]. Despite its common usage in design problems, minimizing total objective 
functions sometimes fails to reflect the competitiveness of a product, especially when the optimal solution achieves a slightly lower cost or environmental impact by significantly reducing the production [197]. Minimizing unit objective functions, however, considers both total behavior and the product quantity, thus providing an opportunity to directly and constructively compare the optimal results with real market values. To that end, the denominators of the unit objective functions are selected as the functional unit of the LCA, the prevalent units on the real market, or the commonly used units in the literature. For instance, the environmental objective in problem (P2) is to minimize the GWP per $1 \mathrm{MJ}$ of energy of biodiesel produced $[192,198]$, and the corresponding economic objective is to minimize the TAC per gasoline gallon equivalent (GGE) of biodiesel produced [21]. In the objective functions of (P2), the variable mtac represents the modified TAC by considering the byproducts as credits, $v^{\text {diesel }}$ stands for the total volume of the biodiesel produced during a year, $m g w p$ is the modified total GWP which accounts for the allocation of surplus electricity, and the denominator $t$ helps determine the unit GWP of algal biodiesel.

$$
\begin{array}{ll}
\min & \text { tac } \\
\min & g w p \\
\text { s.t. } & \text { mass balance constraints } \\
& \text { energy balance constraints } \\
& \text { economic evaluation constraints } \\
& \text { life cycle environmental impact analysis constraints }
\end{array}
$$




$$
\begin{array}{ll}
\min \frac{m t a c}{v^{\text {diesel }}} \\
\min \frac{m g w p}{t}
\end{array}
$$

s.t. mass balance constraints

energy balance constraints

economic evaluation constraints

life cycle environmental impact analysis constraints

Both problems (P1) and (P2) are formulated as bi-criteria, nonconvex MINLP's, which could be computationally demanding for general purpose solvers, due to their combinatorial and nonconvex nature. Therefore, several solution methods are applied to solving these problems. Regarding the objective functions, the $\varepsilon^{-}$ constraint method is applied to transfer the environmental objective function into a new $\varepsilon$-constraint [199]. For (P1), the only nonlinear terms are the exponential sizing equations that evaluate the equipment capital costs. With sizing factors ranging from 0.4 to 0.7 , these sizing equations are actually separable concave functions. An MINLP problem with separable concave functions in the objective function can be effectively handled by a branch-and-refine algorithm [200, 201].

$$
\begin{array}{ll}
\text { min } & o b j=m t a c-Q \cdot v^{\text {diesel }} \\
\text { s.t. } & \text { Original constraints } \\
& m g w p \leq \varepsilon \cdot t \\
& \\
\text { min } & o b j=m t a c-Q \cdot v^{\text {diesel }} \\
\text { s.t. } & \text { Original constraints } \\
& m g w p \leq \varepsilon \cdot t
\end{array}
$$$$
\text { piecewise linear approximation functions }
$$ 


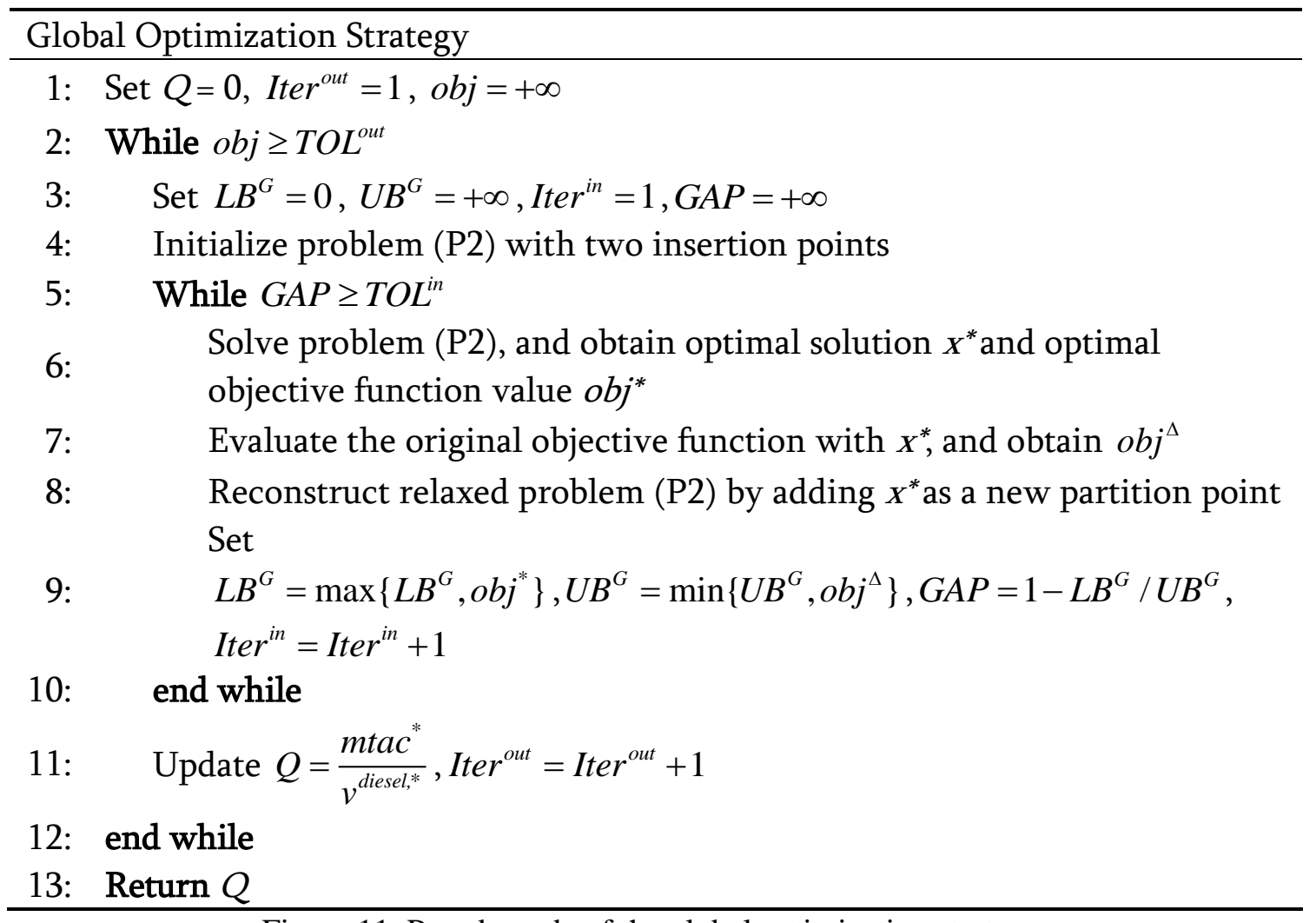
Figure 11. Pseudo-code of the global optimization strategy.

For the model (P2), the additional presence of a fractional term in the economic objective function leads to more computation challenges. In this chapter, the parametric algorithm dedicated to solving problems with fractional terms is applied and integrated with the branch-and-refine algorithm [21]. In the parametric algorithm, we introduce a parameter $Q$ and replace the objective function with $o b j$ as in problem (P3). Consequentially, Newton's method can be applied to search for the optimal objective function value of (P2) [202]. Regarding the remaining nonlinear terms in (P3), we formulate a relaxed, mixed-integer linear programming (MILP) problem (P4) using the proposed piecewise linear 
approximation. As a result, the optimal objective function value of (P4) underestimates that of (P3), but gradually approaches it when the accuracy of the approximation functions increases [21]. In order to properly implement the two algorithms, a global optimization strategy is employed whose pseudo-code is shown in Figure 11. The outer loop implements the parametric algorithm based on Newton's method, while the inner loop implements the branch-and-refine algorithm based on successive piecewise linear approximations to ensure the relaxed formulation achieves enough approximation accuracy. Iter ${ }^{\text {out }}$ and Iter ${ }^{\text {in }}$ are the iteration counters for the outer loop and inner loop, respectively. The absolute optimality tolerances for the outer loop and inner loop are denoted as $T O L^{\text {out }}$ and $T O L^{i n}$, respectively. The current upper bounds and lower bounds are denoted as $L B^{G}$ and $U B^{G}$, respectively. The initial values of $o b j$ and $G A P$ are set to $+\infty$. Thus, this non-convex MINLP problem can be solved by using only an MILP solver [197].

\subsection{Results and discussion}

All the computational experiments are conducted on a Dell Optiplex 790 desktop with Intel(R) Core(TM) i5-2400 3.10GHz CPU, 8GB RAM and Windows 7 64-bit operating system. All the models are coded in GAMS 24.3.1. [203] Specifically, the MILP solver employed is CPLEX 12.6, while the MINLP solvers utilized are 
BARON 14.0 [204], and SCIP 3.1. The relative optimality tolerances of BARON 14.0 [204] and SCIP 3.1, and the absolute optimality tolerances for both the parametric algorithm and the branch-and-refine algorithm are all set to $10^{-6}$.

\subsubsection{Environmental life cycle analysis and optimization results for manufacturing bioproducts and biodiesel from microalgae}

In this section, we present the life cycle environmental performance of producing biodiesel and each type of bioproduct from microalgae, and compare the optimization results with the results from the literature.

Table 2. Life cycle $\mathrm{CO}_{2}$ equivalent emissions from hydrogen production.

\begin{tabular}{ccc}
\hline Hydrogen production method & $\begin{array}{c}\mathrm{GWP}\left(\mathrm{kg} \mathrm{CO}_{2}-\mathrm{eq} / \mathrm{kg}\right. \\
\left.\mathrm{H}_{2}\right)\end{array}$ & Source \\
\hline $\begin{array}{c}\text { Steam reforming of } \mathrm{CH}_{4} \\
\text { Total Autocatalytic decomposition } \\
\text { of } \mathrm{CH}_{4}\end{array}$ & 11.59 & Literature \\
$\begin{array}{c}\text { Electrolysis cell with renewable } \\
\text { energy sources }\end{array}$ & 1.10 & $\begin{array}{c}\text { Literature } \\
{[206]}\end{array}$ \\
Steam reforming of glycerol & 2.54 & This work \\
\hline
\end{tabular}

If the bioproduct manufacturing section is devoted to hydrogen production, the lowest unit GWP obtained is $4.28 \mathrm{~kg} \mathrm{CO}$-eq $/ \mathrm{kg} \mathrm{H}$ and the corresponding optimal process selects pressure filtration in the harvesting section, hexane as the lipid extractant, direct combustion for biogas utilization, enzyme-catalyzed transesterification for biofuel production, and glycerol steam reforming to produce 
hydrogen. The life cycle environmental impacts of hydrogen production have been studied by several researchers, and the unit GWP ranges approximately from 1 to $12 \mathrm{~kg} \mathrm{CO} 2$-eq $/ \mathrm{kg} \mathrm{H}_{2}$, as shown in Table 2 . Hydrogen production by steam reforming of glycerol demonstrates significant environmental advantages over the traditional fossil-based method, reaching a life cycle GHG reduction of over $63 \%$ of the total $\mathrm{CO}_{2}$ equivalent emissions. Although autocatalytic decomposition and electrolysis exhibit even lower unit GWP, these methods lack technological readiness and tend to be less cost-effective at a large scale.

If we fix PG as the only bioproduct produced, the minimum unit GWP is shown in Figure 12 along with environmental impact evaluation results from industry. We note that petroleum-based PG production contributes as much as $3.75 \mathrm{~kg} \mathrm{CO}$-eq life cycle emissions per kg of PG produced. Two commercialized bio-based process from $\operatorname{ADM}(\mathrm{R})$ and Zemea $(\mathrm{R})$ manage to reduce the unit GWP to $86.4 \%$ and $58.1 \%$ of the environmental impact of the petroleum-based counterpart [207, 208]. Benefiting from the environmentally sustainable algal biodiesel conversion process, the unit GWP of $1.82 \mathrm{~kg} \mathrm{CO}$-eq/ $\mathrm{kg}$ glycerol-based PG in this research achieves a 51.5\% GHG reduction by selecting a similar upstream process configuration to the environmentally optimal process for hydrogen production. 


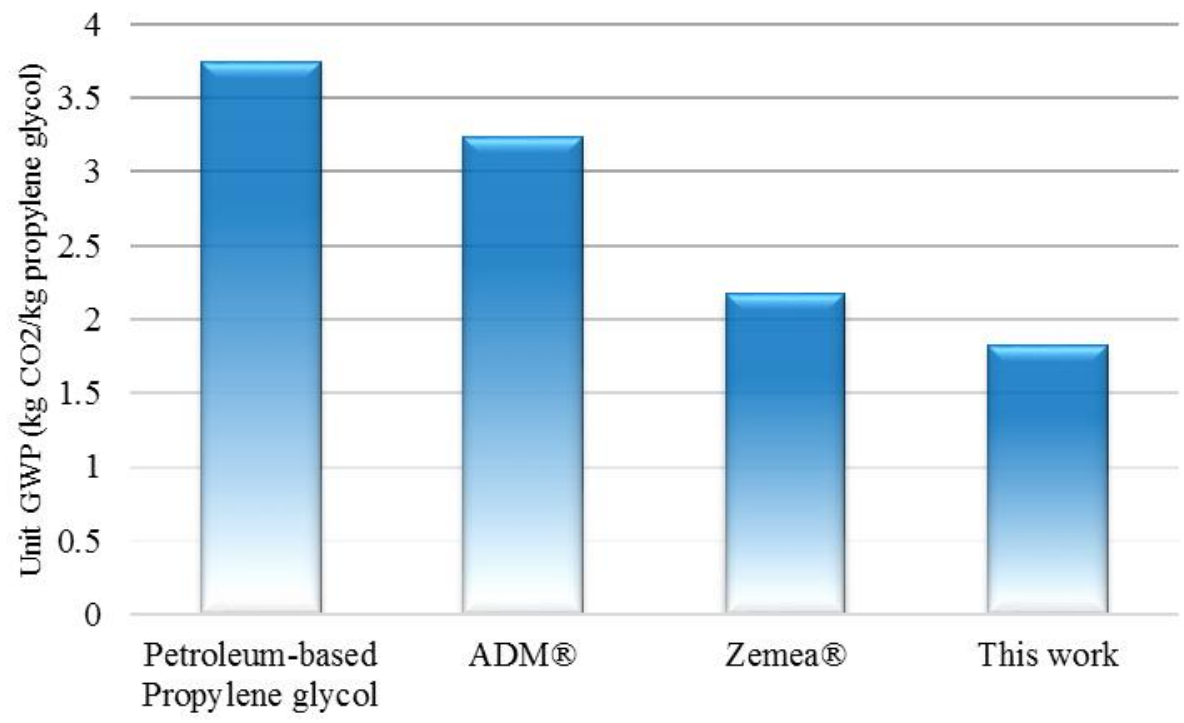

Figure 12. Life cycle $\mathrm{CO}_{2}$ equivalent emissions from $\mathrm{PG}$ production.

Very little attention has been drawn to the LCA of glycerol ether products.

Beatrice et al. conducted a preliminary LCA on the etherification of glycerol with tert-butyl alcohol and isobutylene, but sparse details were reported with respect to the specific life cycle GHG emissions of their GE's [209]. After optimizing the bioproduct process focusing on GE synthesis, we obtain a unit GWP of $2.37 \mathrm{~kg}$ $\mathrm{CO}_{2}-\mathrm{eq} / \mathrm{kg} \mathrm{GE}$ produced. We are confident that the corresponding optimal process, which selects the same upstream processes as in the previous scenarios will be environmentally attractive and worthy of investment.

For the case of PHB production, the optimal unit GWP is $4.10 \mathrm{~kg} \mathrm{CO}-\mathrm{eq} / \mathrm{kg} \mathrm{PHB}$ with the same upstream process configuration mentioned in the above three bioproduct production scenarios. In contrast, Rostkowski et al. reported a unit 
GWP of $942 \mathrm{~kg} \mathrm{CO}$-eq/kg PHB produced from waste biogas [189]. The environmental performance of producing polyhydroxyalkanoates from corn-based PHA fermentation and recovery by Kim et al. fell in the range of $1.6-4.1 \mathrm{~kg} \mathrm{CO}_{2}-$ eq/kg PHA [210]. In terms of the fossil based products, polypropylene, low density polyethylene, and high impact polystyrene were studied, resulting in unit GWP's of $4.34,3.88$, and $6.89 \mathrm{~kg} \mathrm{CO}$-eq/kg plastic, respectively [211]. Through a comparison with these literature data, it is apparent that PHB production from algal glycerol is environmentally competitive.

In terms of diesel production, the unit GWP's of producing petroleum-based diesel and soy-derived biodiesel are 0.12 and $0.025 \mathrm{~kg} \mathrm{CO}_{2}$-eq/ $\mathrm{MJ}$ diesel, respectively [192]. In the current work, the production of bioproducts and biodiesel results in a minimum unit GWP of $0.040 \mathrm{CO}_{2}$-eq/ MJ biodiesel, which can be considered at least equally environmentally sustainable to other biomass-based diesel products, and outperforms the life cycle environmental impacts of manufacturing fossilbased products. Accordingly, the environmental impact breakdown is shown in Figure 13. The manufacturing stage stands out to be the largest contributor to GWP. Specifically, the gas emissions from algae cultivation account for more than $95 \%$ of the GWP in this category and heavily affect the environmental impacts of the entire process. In the mass balance constraints, we assume the carbon dioxide 
utilization efficiency to be $75 \%$, which is a conservative approximation mentioned by Frank and coworkers [159]. Therefore, an advanced cultivation system fostering a robust and efficient algal strain and enabling an improvement of the carbon utilization efficiency, could substantially reduce GHG emissions.

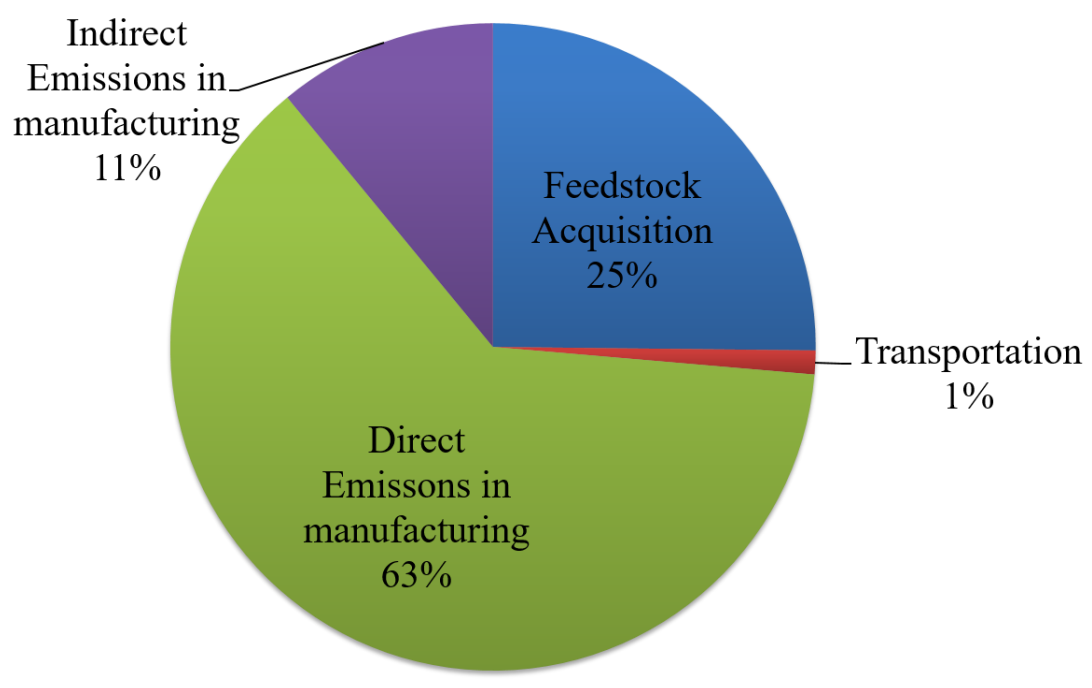

Figure 13. Total GWP breakdown for the minimum unit GWP of biodiesel.

From the above results, bioproducts and biodiesel production from the proposed superstructure proves to be environmentally sustainable, especially compared to fossil-based chemical products. The excellent performance stems primarily from two reasons. First, when biogas derived from lipid extracted biomass is chosen to generate electricity and reusable off-gas, there is a significant reduction in GWP from selling surplus electricity, and the total environmental impacts also benefit from fewer direct GHG emissions. Secondly, the unit GWP is calculated to take 
into account co-product allocation, averaging the environmental benefit of the entire process and further reducing the unit environmental impacts.

\subsubsection{Economic savings for biofuel production}

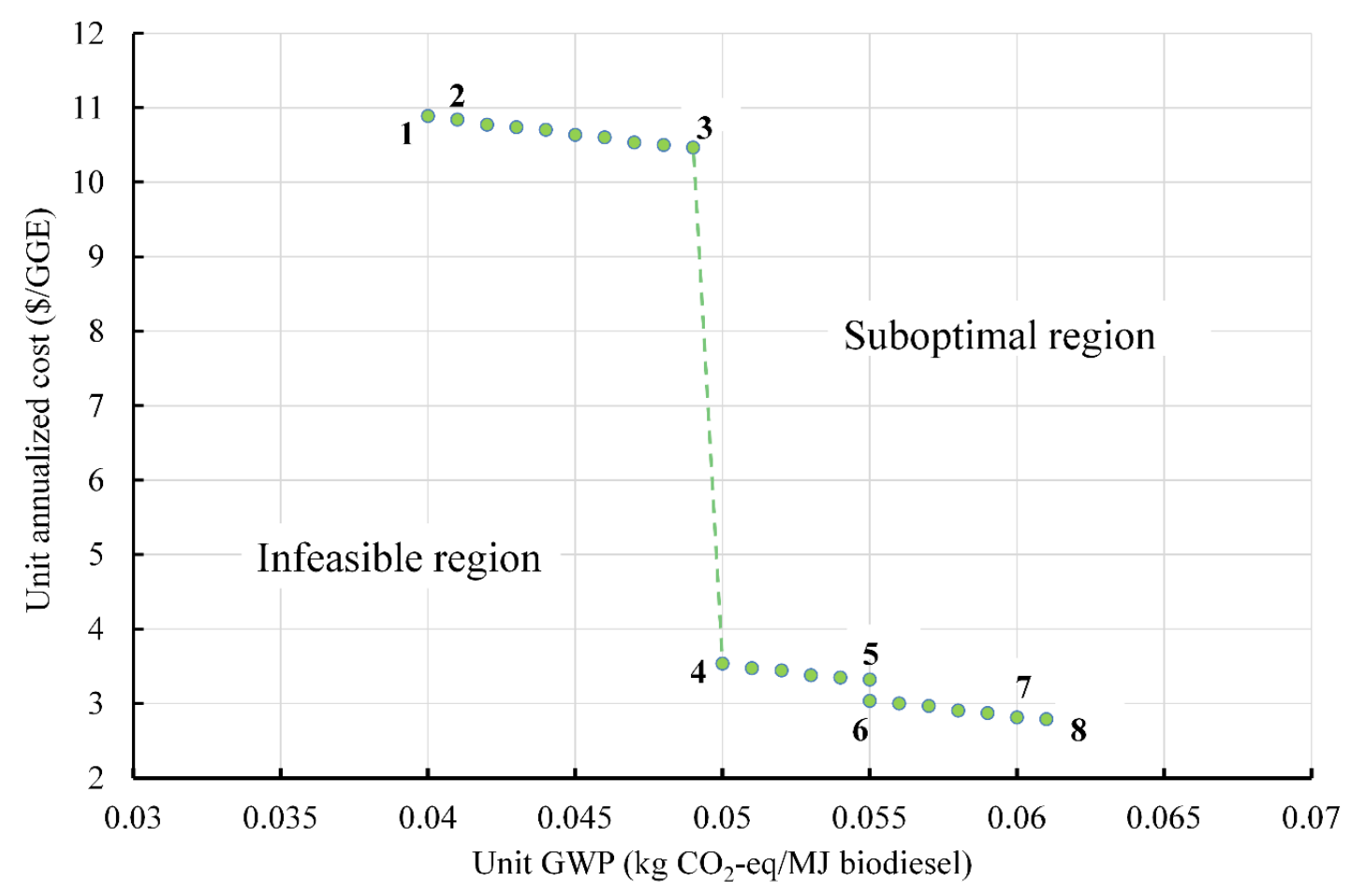

Figure 14. Pareto-optimal curve associated with the UAC and unit GWP.

In the existing literature, reported costs for algal biodiesel production do not align with the goal of cost-competitive biofuels [20,21, 41]. The unit production cost in this chapter, however, is substantially reduced by producing value-added bioproduct alongside biodiesel from microalgae. Before further conclusions are drawn, a multiobjective MINLP model is solved to minimize unit biodiesel production cost and unit GWP simultaneously, and a Pareto-optimal profile is 
generated with the global optimization strategy and the $\varepsilon$-constraint method. As shown in Figure 14, the Pareto-optimal profile separates the plane into a suboptimal region and an infeasible region. Overall, the curve demonstrates the trade-off between two competing objective functions: that is, the minimum unit annualized cost (UAC) decreases when the corresponding minimum unit GWP increases. Also, the UAC drops significantly between points 3 and 4, indicating that the UAC is sensitive to certain changes in technology selections.

With no technology presented in the cell disruption and hydrogen production sections, the most environmentally sustainable process selected by point 1 employs pressure filtration in the harvesting section, hexane as the lipid extractant, direct combustion for biogas utilization, enzyme-catalyzed transesterification for biofuel production, and GE synthesis for bioproduct manufacturing. The minimum unit GWP of this process is $0.040 \mathrm{~kg} \mathrm{CO}$-eq/MJ biodiesel, but we must shoulder a UAC of $\$ 10.89 /$ GGE in exchange for the environmental savings. In contrast, the optimal process for points 4 to 5 (Figure 15) favors supercritical carbon dioxide in the lipid extraction section. As mentioned previously, the selection of this technology could lead to considerable unit cost reduction, but gently impact the environment. Eventually, the optimal process selected by point 8 achieves a UAC of $\$ 2.79 /$ GGE and a unit GWP of $0.061 \mathrm{~kg} \mathrm{CO} \mathrm{CO}_{2}$-eq/MJ biodiesel by using centrifugation and 
heterogeneously catalyzed transesterification. Overall, this result has a $74 \%$ decrease in UAC with a $34 \%$ increase in GWP, which shows that significantly larger savings in UAC can be obtained than the increase in unit GWP.

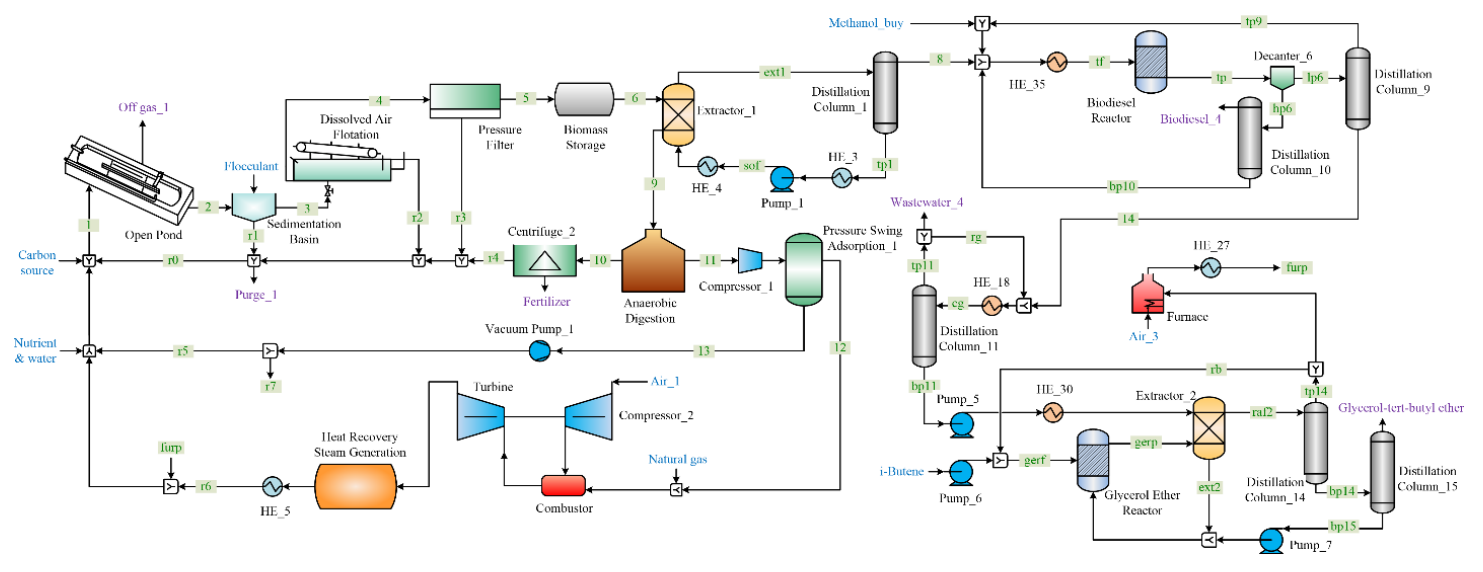

Figure 15. Optimal process flow diagram of point 4.

The optimal economic and environmental performance on the Pareto-optimal curve depends not only on technology selection, but also on the strategy to satisfy utility consumption. Points 2 and 3 select the same process configuration, but differ in the amount of natural gas purchased for steam generation. If heat consumption is satisfied by burning natural gas, a large proportion of the resulting off-gas can be reused and the environmental impact associated with the heat should be much lower than that of purchased steam. However, equipment construction for the steam generation system is costly, so as more heat is generated on site, the less economically viable a process will be. 
The economic and environmental performance, as well as the throughputs of the four extreme points, corresponding to minimum total GWP, minimum total cost, minimum unit GWP, and minimum unit cost are presented in Table 3. From the Pareto-optimal point of view, the first two solutions locate in the suboptimal region and represent two feasible but non-optimal solutions. Note that the UAC is defined as the ratio of the modified TAC to the biodiesel throughput, which should be different from the ratio of TAC to the throughput. Similarly, when we calculate the unit GWP, the environmental impact allocation is taken into consideration to isolate the contribution caused solely by the biodiesel product. According to the U.S. Department of Energy [212], the biodiesel price in April, 2014 was \$4.01/gallon, or \$4.18/GGE. From the Pareto-optimal curve, the optimal biodiesel production costs for the strategy of co-production of bioproducts outlined in this chapter, ranging from $\$ 3.54 / \mathrm{GGE}$ and $\$ 2.79 / \mathrm{GGE}$ for points 4 to 8 , are costcompetitive.

Table 3. Performance of four extreme points. The minimum value in each row is highlighted in bold.

\begin{tabular}{ccccc}
\hline & $\begin{array}{c}\text { Minimu } \\
\text { m total } \\
\text { GWP }\end{array}$ & $\begin{array}{c}\text { Minimu } \\
\text { m TAC }\end{array}$ & $\begin{array}{c}\text { Minimu } \\
\text { m unit } \\
\text { GWP }\end{array}$ & $\begin{array}{c}\text { Minimu } \\
\text { m UAC }\end{array}$ \\
\hline TAC $(\$ M M)$ & 224.07 & 207.04 & 289.29 & 240.56 \\
Total GWP $\left(\mathrm{kt} \mathrm{CO}_{2}\right.$-eq $)$ & $1,011.25$ & $1,052.12$ & $1,143.16$ & $1,074.59$ \\
UAC $(\$ / G G E)$ & 3.47 & 3.35 & 10.85 & 2.79 \\
Unit GWP $(\mathrm{kg} \mathrm{CO}$-eq/ MJ & 0.064 & 0.075 & 0.040 & 0.061
\end{tabular}


biodiesel)

$\begin{array}{lllll}\text { Biodiesel throughput (million GGE) } & 48.07 & 47.27 & 18.73 & 47.39\end{array}$

\subsubsection{Computational results}

In this subsection, we compare the computation performance of the four extreme points, solved by BARON 14.0 [204], SCIP 3.1, and the proposed global optimization strategy. The model of minimizing the UAC by BARON 14.0 [204] and SCIP 3.1 consists of 23 discrete variables, 9,185 continuous variables, and 9801 constraints, while the number of continuous variables and constraints slightly increases when the problem is solved by the proposed global optimization strategy.

Table 4. Computation comparison for four extreme points on the Pareto-optimal curves.

\begin{tabular}{|c|c|c|c|c|c|c|c|}
\hline \multirow{2}{*}{$\begin{array}{l}\text { Objective } \\
\text { function }\end{array}$} & \multicolumn{2}{|c|}{$\begin{array}{c}\text { BARON } 14.0 \\
{[204]}\end{array}$} & \multicolumn{2}{|c|}{ SCIP 3.1} & \multicolumn{3}{|c|}{ proposed solution methods } \\
\hline & $\begin{array}{l}\text { Optimal } \\
\text { value }\end{array}$ & $\mathrm{CPU}(\mathrm{s})$ & $\begin{array}{l}\text { Optimal } \\
\text { value }\end{array}$ & $\mathrm{CPU}(\mathrm{s})$ & $\begin{array}{l}\text { Optimal } \\
\text { value }\end{array}$ & $\mathrm{CPU}(\mathrm{s})$ & Iter. \\
\hline $\begin{array}{l}\text { Total GWP } \\
\text { (kt } \mathrm{CO}_{2} \text {-eq) }\end{array}$ & $1,011.25$ & 3.37 & $1,011.25$ & 0.32 & $1,011.25$ & 0.25 & 1 \\
\hline TAC (\$MM) & 207.04 & 290 & 207.04 & 4.52 & 207.04 & 19.34 & 6 \\
\hline $\begin{array}{l}\text { (kg CO} \mathrm{CO}_{2} \text {-eq/ } \\
\text { MJ biodiesel) }\end{array}$ & 0.04 & 3,435 & $\mathrm{NA}^{\mathrm{a}}$ & 3,600 & 0.04 & 0.92 & 4 \\
\hline $\begin{array}{c}\mathrm{UAC} \\
(\$ / G G E)\end{array}$ & 2.79 & 3,275 & 2.79 & 23.33 & 2.79 & 11.06 & $5-5^{b}$ \\
\hline
\end{tabular}

a. The calculation is terminated with "intermediate infeasible". A lower bound of 0.012 is returned.

b. This point is solved with two outer iterations by parametric algorithm, costing five inner iterations each. 
As listed in Table 4, the problem of minimizing total GWP can be readily solved by all three methods since this problem is simply an MILP. In the remaining cases, BARON 14.0 [204] is able to provide global optimal solutions in longer computation times. In contrast, the proposed solution methods are able to obtain optimal solutions in seconds. SCIP 3.1 is efficient when solving problems with economic objective functions, but is unable to provide optimal information for the unit GWP problem within 3,600 seconds. Overall, the proposed solution methods could considerably improve computation efficiency when globally optimizing problems with separable concave terms, or together with a fractional term in the objective function. For a similar nonconvex problem with a much larger size, general purpose solvers would hardly be able to return global optimal solutions in reasonable computation times, whereas the proposed method solves only linear problems during each iteration and still holds promise to obtain feasible solutions close to the global optimality [200,213].

\subsection{Summary}

In this paper, we proposed by far the most comprehensive superstructure for the production of biodiesel and four bioproducts, including hydrogen, PG, GE, and PHB from microalgae. Based on the proposed superstructure, we conducted a cradle-to-gate LCA and developed a bi-criteria MINLP model to simultaneously 
optimize the environmental and economic performance following the life cycle optimization framework. The computation efficiency was enhanced with a tailored global optimization strategy. In terms of the environmental impacts, manufacturing algal bioproducts resulted in reduction of unit life cycle GHG emissions by $5 \%$ to $63 \%$, compared with the petrochemical counterparts. The coproduction of value-added bioproducts from algal glycerol helped reduce the biodiesel production cost to as low as $\$ 2.79 /$ GGE by employing centrifugation in the harvesting section, supercritical carbon dioxide as the lipid extractant, direct combustion for biogas utilization, heterogeneously catalyzed transesterification for biofuel production, and GE synthesis in bioproduct production. Computational results show that the tailored solution methods can significantly reduce the computational times of solving the proposed nonconvex MINLP problems. 


\section{CHAPTER 3}

\section{ENERGY SYSTEM DESIGN UNDER UNCERTAINTY}

\subsection{Introduction}

In this chapter, we develop a comprehensive processing network to produce biofuels and value-added bioproducts from microalgae. A variety of technology and process alternatives are incorporated into the network. The superstructure consists of eight sections: cultivation, harvesting, dewatering, cell disruption, lipid extraction, biofuel production, upgrading, and remnant treatment. The biofuels that can be produced from the network include biodiesel and renewable diesel, and the bioproducts include hydrogen, propylene glycol, glycerol-tert-butyl ether, and poly-3-hydroxybutyrate (PHB). With a total of 46,704 alternative processing pathways, the processing network represents by far the largest superstructure for producing algal fuels and bioproducts. Since uncertainty usually exists in network design problems for algae-based processes, the deterministic optimal solution that ignores uncertainty may lead to suboptimal or even infeasible solutions in some uncertainty realizations. Therefore, it is critical to handle uncertainty and determine the robust optimal processing pathway using a systematic approach. We develop a two-stage adaptive robust mixed integer fractional programming (ARMIFP) model to cope with uncertainty and determine the robust optimal 
processing pathway with the highest return on investment (ROI). Since the proposed problem cannot be solved directly by any existing solver or method, we develop a solution method that integrates a parametric algorithm with a columnand-constraint generation algorithm. We also investigate the maximum productivity under uncertainty via two-stage adaptive robust optimization. The obtained robust optimal processing pathway reveals the bottleneck for future development of algal processes and technologies.

The novelties of this work are summarized below:

- We develop the most comprehensive processing network by far for producing biofuels and value-added bioproducts from microalgae with 46,704 possible processing pathways;

- A two-stage ARMIFP model is developed to maximize the ROI under uncertainty;

- Since the proposed problem cannot be solved by any off-the-shelf solver, we develop an efficient solution method that integrates a parametric algorithm for handling the mixed-integer fractional objective with a column-and-constraint generation algorithm for solving an auxiliary two-stage adaptive robust mixed integer linear programming (MILP) problem.

The rest of this article is organized as follows. In the next section, we describe the structure and technologies in the proposed network. The formulations of a deterministic mixed integer fractional programming (MIFP) model, an uncertainty set, and a two-stage ARMIFP model are given in the following section. In the 
Solution Strategy section, we present a solution method for solving the two-stage ARMIFP problem. We present the optimal solutions in the Results and Discussion section. The conclusion is given at the end of the article.

\subsection{Network for producing biofuels and bioproducts from}

\section{microalgae}

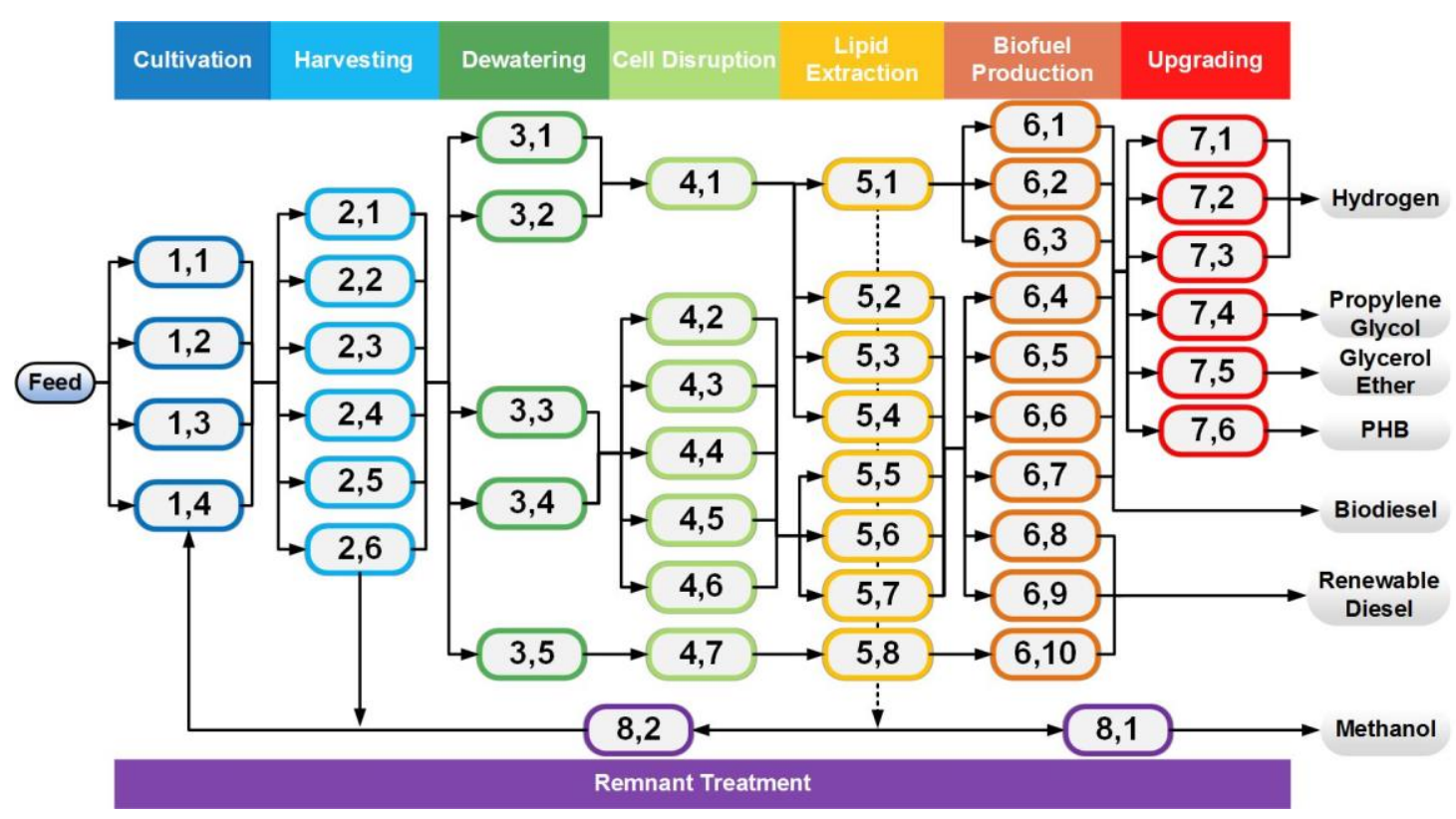

Figure 16. Network for producing biofuels and bioproducts from microalgae. $<1,1>$ open pond; $<1,2>$ flat plate PBR; $<1,3>$ bubble column PBR; $<1,4>$ tubular PBR; $<2,1>$ flocculation with poly-electrolyte; $\langle 2,2\rangle$ flocculation with sodium hydroxide; $\langle 2,3\rangle$ flocculation with polyaluminum chloride; $\langle 2,4\rangle$ flocculation with aluminum sulfate; $\langle 2,5\rangle$ flocculation with chitosan; $\langle 2,6\rangle$ flocculation with poly- $\gamma$-glutamic acid; $\langle 3,1>$ freeze drying; $<3,2>$ thermal drying; $\langle 3,3\rangle$ pressure filtration; $\langle 3,4\rangle$ centrifugation; $\langle 3,5\rangle$ blank; $\langle 4,1\rangle$ bead beating;

$\langle 4,2\rangle$ bead beating; $\langle 4,3\rangle$ microwaving; $\langle 4,4\rangle$ high pressure homogenization; $\langle 4,5\rangle$

sonication; $\langle 4,6\rangle$ blank; $\langle 4,7\rangle$ HTL; $\langle 5,1\rangle$ blank; $\langle 5,2\rangle$ hexane extraction; $\langle 5,3\rangle$ isopropanol/hexane extraction; $\langle 5,4\rangle$ supercritical $\mathrm{CO}_{2}$ extraction; $\langle 5,5\rangle$ hexane extraction; $<5,6>$ butanol extraction; $\langle 5,7\rangle$ supercritical $\mathrm{CO}_{2}$ extraction; $\langle 5,8\rangle$ blank; $\langle 6,1\rangle$ alkaline in-

situ transesterification; $\langle 6,2\rangle$ acidic in-situ transesterification; $\langle 6,3\rangle$ enzymatic in-situ transesterification; $\langle 6,4\rangle$ sodium-methoxide-catalyzed transesterification; $\langle 6,5\rangle$ heterogeneous acid-catalyzed transesterification; $\langle 6.6\rangle$ supercritical methanol transesterification; $\langle 6,7\rangle$ enzymatic transesterification; $\langle 6,8\rangle \mathrm{Co} / \mathrm{Mo}-$ catalyzed hydroprocessing; <6,9> Ni/Mo-catalyzed hydroprocessing; <6,10> HZSM-5-catalyzed 
hydroprocessing; $\langle 7,1\rangle$ steam reforming; $\langle 7,2\rangle$ autothermal reforming; $\langle 7,3\rangle$ aqueous-phase reforming; $\langle 7,4\rangle$ propylene glycol production; $\langle 7,5\rangle$ glycerol-tert-butyl ether production; $<7,6>$ PHB production; $<8,1>$ methanol production; $<8,2>$ direct combustion.

As shown in Figure 16, we propose by far the most comprehensive processing network with 46,704 processing pathways to produce a variety of biofuels and value-added bioproducts from microalgae. The major feedstock of the proposed network is a gaseous carbon source that can be ultimately converted to biodiesel, renewable diesel, hydrogen, propylene glycol, glycerol-tert-butyl ether, and PHB. The microalgae Chlorella vulgaris with $25 \mathrm{wt} \%$ lipid is considered in this system [159]. There are eight sections in the network: cultivation, harvesting, dewatering, cell disruption, lipid extraction, biofuel production, upgrading, and remnant treatment. The first seven sections were introduced in a previous study [21]. In the proposed network, we combine the primary dewatering and secondary dewatering sections into one dewatering section, consider more technology alternatives in the remnant treatment section, and incorporate a new section for the conversion of raw glycerol to algal bioproducts. Detailed superstructure configuration description is presented in Appendix A.

\subsection{Problem statement}

In this section, we formally state the problem addressed in this chapter. We propose a comprehensive superstructure network for producing algal fuels and 
bioproducts as shown in Figure 16. A plethora of technology alternatives are incorporated into the network. The parameters involved in the problem are listed below.

- The upper and lower bounds of the capacity of each technology;

- The composition and total mass flow rate of the feed gas;

- Component concentrations in the inlet flows;

- The conversion of each reaction;

- Stoichiometric coefficients of each reaction;

- The split fraction of each species to various flows;

- Unit consumption and generation rates of power, heating, and cooling utilities for each technology;

- The area productivity of each bioreactor;

- Operating hours of each technology in a year;

- Base case capital costs and mass flow rates for all technologies;

- Coefficients for evaluating total project investment based on capital costs;

- Coefficients for evaluating operating costs;

The uncertain parameters are modeled using an uncertainty set. Each uncertain parameter is constrained by an upper bound and a lower bound, the values of which can be retrieved from historical records. The nominal value of each uncertain parameter takes the average value of the upper and lower bounds. Moreover, a budget of uncertainty is introduced in the uncertainty set to control the level of conservatism of the resulting optimal solution.

Further assumptions of the problem include: 
- The algae growth rate remains constant during a year;

- The distribution of products from anaerobically digesting remnants from different transesterification technologies is identical;

- The technology capital costs are modeled as piecewise linear functions of corresponding capacities;

- Given that several bioproducts can be coproduced in the superstructure, we assume there are markets to sell bioproducts into;

The goal of this research is to determine the robust optimal processing pathways for producing algal fuel and bioproduct under uncertainty. The objective function is to maximize ROI. The mathematical model accounts for network configuration, mass balance, and economic evaluation. The major decision variables include:

- Technology selection;

- The capacity of each technology;

- The operating mass flow rate of each technology;

- The worst-case uncertainty realization.

\subsection{Model formulation}

\subsubsection{Deterministic model}

We first develop a deterministic problem that maximizes the ROI of producing algal fuels and bioproducts. The general model formulation (DM) is shown below. There are three groups of constraints, namely network configuration constraints, mass balance constraints, and economic evaluation constraints. Integer variables are introduced in network configuration constraints to model the selection of 
technology alternatives in the algae conversion network. All the constraints are mixed-integer linear equalities or inequalities. The nonlinear term in the deterministic problem is the fractional objective function. The indices for sections, technology alternatives, and species are denoted as $i, j$, and $k$, respectively. The variables are denoted as upper-case letters, whereas parameters are denoted as lower-case letters.

(DM) $\quad \max \quad R O I$ as defined in (1.B13)

s.t. network configuration constraints (1.1)-(1.5)

mass balance constraints (1.7)-(1.22)

economic evaluation constraints (1.23)-(1.27), (1.B1)-(1.B12)

$$
\begin{aligned}
& \sum_{j \in J} Y_{i, j}=1, \quad \forall i \in I \\
& Y_{3,1}+Y_{3,2}=Y_{4,1} \\
& Y_{3,5}=Y_{4,7}=Y_{5,8}=Y_{6,10} \\
& Y_{4,1}=Y_{5,1}+Y_{5,2}+Y_{5,3}+Y_{5,4} \\
& Y_{5,1}=Y_{6,1}+Y_{6,2}+Y_{6,3}
\end{aligned}
$$

The network configuration is described by Equations (1.1)-(1.5). Following existing models for superstructure optimization $[14,16,214,215]$, binary variable $Y_{i, j}$ is introduced to select technology $j$ in section $i$ in the network. $Y_{i, j}$ is equal to 1 if the corresponding technology is selected, and 0 otherwise. Equation (1.1) guarantees that one and only one technology is selected in each section in the network. Equation (1.2) requires that if either freeze drying or thermal drying is selected, 
bead beating has to be selected. Equation (1.3) requires HTL, HZSM-5-catalyzed hydroprocessing and associated blank technologies to be selected simultaneously. Equation (1.4) requires that if bead beating is selected, only the blank technology (bypass), hexane extraction, isopropanol/hexane extraction, and supercritical $\mathrm{CO}_{2}$ extraction can be selected in the next section. Likewise, Equation (1.5) requires that if no technology is selected in the extraction section, the algae slurry can be sent to only alkaline in-situ transesterification, acidic in-situ transesterification, and enzymatic in-situ transesterification in the next section. Since the any algal remnant can be processed by either methanol production or direct combustion, all lipid extraction technologies are connected to both remnant treatment technologies and no connection restriction between these sections is enforced.

$$
\begin{aligned}
& 0 \leq \sum_{k \in K} Q O_{i, j, k} \leq Y_{i, j} \cdot u b o_{i, j}, \quad \forall i \in I, j \in J \\
& 0 \leq \sum_{k \in K} Q I_{i, j, k} \leq Y_{i, j} \cdot u b i_{i, j}, \quad \forall i \in I, j \in J \\
& M_{i, j, k}^{\text {out }} \leq Q O_{i, j, k}, \quad \forall i \in I, j \in J, k \in K \\
& M_{i, j, k}^{\text {in }} \leq Q I_{i, j, k}, \quad \forall i \in I, j \in J, k \in K
\end{aligned}
$$

Mass balances are described by Constraints (1.7)-(1.22). The upper and lower bounds of the technology capacity are defined by Constraints (1.7) and (1.6). Nonnegative variables $Q I_{i, j, k}$ and $Q O_{i, j, k}$ are the capacities of species $k$ in technology $j$ in section $i$ for the "in" and "out" flows, respectively. The definitions of "in" and 
"out" flows are given in Figure 17. $u b o_{i, j}$ and $u b i_{i, j}$ are the upper bounds of the capacities for the "in" and "out" flows of technology $j$ in section $i$, respectively. If technology $j$ in section $i$ is not selected, or $Y_{i, j}=0$, the corresponding capacities will only be 0 . Linking constraints (1.9) and (1.8) enforce that the "in" and "out" flow rates should not be greater than the corresponding capacities. The introduction of technology capacities in addition to operating flow rates facilitates the modeling of first-stage decisions and second-stage decisions in the two-stage adaptive robust optimization model as detailed in the latter subsections.

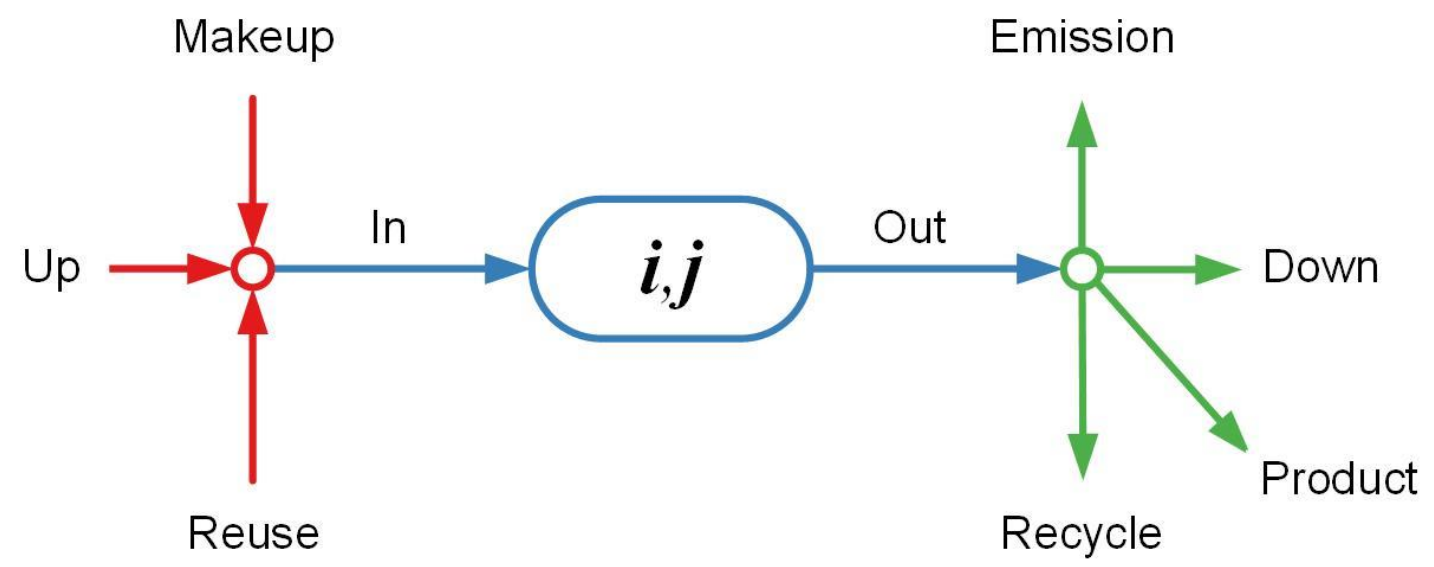

Figure 17. General flow relationships of technology $j$ in section $i$ in the mass balance.

$$
\begin{aligned}
& \sum_{j \in J} M_{l, j, k}^{u p}-w f_{k}^{\text {feed }} \cdot M^{\text {feed }}=0, \quad \forall k \in K \\
& \sum_{j \in J} M_{i, j, k}^{u p}-p_{i}^{\text {down }} \cdot \sum_{i^{\prime} \in I_{i}^{l p}, j \in J} M_{i^{\prime}, j, k}^{\text {down }}=0, \quad \forall 2 \leq i \leq 8, k \in K \\
& \sum_{j \in J} M_{i_{i, j}}^{\text {reuse }}-\sum_{i^{\prime} \in i_{i}^{\text {Iese }}, j \in J} M_{i^{\prime}, j, k}^{\text {recycle }}=0, \quad \forall i \in I, k \in K
\end{aligned}
$$




$$
\begin{aligned}
& M_{i, j, k}^{i n}-\sum_{k^{\prime} \in K} p_{i, j, k, k^{\prime}}^{i n} \cdot M_{i, j, k^{\prime}}^{i n}=0, \quad \forall i \in I, j \in J, k \in K \\
& M_{i, j, k}^{i n}-M_{i, j, k}^{u p}-M_{i, j, k}^{\text {reuse }}-M_{i, j, k}^{\text {makeup }}=0, \quad \forall i \in I, j \in J, k \in K
\end{aligned}
$$

The relationships between flow rate variables are based on the generic mass balance framework introduced in the previous work [21]. As shown in Figure 17, nine flows describe the mass balance for technology $j$ in section $i$. The mass balance is specified by three steps: an inlet converging step (red arrows, Equations $(1.10)-(1.14))$, a conversion step (blue arrows, Equation (1.15)), and an outlet separation step (green arrows, Equations (1.16)-(1.19)). The "up" flow rate of the first section is specified by multiplying the feed composition $w f_{k}^{\text {feed }}$ by the total feed flow rate $M^{\text {feed }}$. In the remaining sections, the "up" flow rate of section $i$ is the sum of several "down" flow rates, as specified by set $I_{i}^{u p} \cdot p_{i}^{\text {down }}$ takes biomass storage into account since microalgae stop growing at night [20]. Following a similar approach, the "reuse" flow rate of section $i$ is the sum of several "recycle" flow rates, as specified by set $I_{i}^{\text {reuse }}$. The feedstock relationship is described by Equation (1.13), where $p_{i, j, k, k^{\prime}}^{i n}$ is the concentration of species $k$ in the "in" flow of technology $j$ in section $i$ with respect to other species $k$ 'in the same flow. The purpose of Equation (1.13) is to calculate the mass flow rates of "makeup" flows with fixed material distribution data in the corresponding "in" flows. Finally in 
Equation (1.14), the "in" flow rate is the sum of corresponding "up", "reuse", and "makeup" flow rates.

$$
M_{i, j, k}^{\text {out }}-M_{i, j, k}^{i n}-\sum_{k^{\prime} \in K}\left(s c_{i, j, k, k^{\prime}} \cdot x_{i, j} \cdot M_{i, j, k^{\prime}}^{i n}\right)=0, \quad \forall i \in I, j \in J, k \in K
$$

The conversion step is described by Equation (1.15), where $s c_{i, j, k, k^{\prime}}$ is the stoichiometric coefficient of species $k$ based on another species $k^{\prime}$ and $x_{i, j}$ is the conversion for technology $j$ in section $i$.

$$
\begin{aligned}
& M_{i, j, k}^{\text {out }}-M_{i, j, k}^{\text {down }}-M_{i, j, k}^{\text {recycle }}-M_{i, j, k}^{\text {emission }}-M_{i, j, k}^{\text {product }}=0, \quad \forall i \in I, j \in J, k \in K \\
& M_{i, j, k}^{\text {down }}-\sum_{k^{\prime} \in K}\left(s f_{i, j, k, k^{\prime}}^{\text {down }} M_{i, j, k^{\prime}}^{\text {out }}\right)=0, \quad \forall i \in I, j \in J, k \in K \\
& M_{i, j, k}^{\text {emission }}-s f_{i, j, k}^{\text {emissionl }} \cdot M_{i, j, k}^{\text {out }}+s f_{i, j, k}^{\text {emission }} M_{i, j, k}^{\text {down }}=0, \quad \forall i \in I, j \in J, k \in K \\
& M_{i, j, k}^{\text {product }}-s f_{i, j, k}^{\text {product }} \cdot M_{i, j, k}^{\text {out }}=0, \quad \forall i \in I, j \in J, k \in K \\
& M^{\text {feed }} \leq f u \\
& M_{i, j, k}^{\text {makeup }} \leq m u_{i, j, k}, \quad \forall i \in I, j \in J, k \in K \\
& \sum_{i \in I, j \in J} M_{i, j, k}^{\text {product }}=p d_{k}, \quad \forall k \in K P
\end{aligned}
$$

In the outlet separation step, the "out" flow is split into four flows, namely "down", "recycle", "emission", and "product" flows. The "down" flow rate is defined by Equation (1.17), where $s f_{i, j, k, k^{\prime}}^{\text {down }}$ is the split fraction of species $k$ based on another species $k^{\prime}$ for technology $j$ in section $i$. The model in Equation (1.17) is advantageous when the concentration is fixed in the "down" flow but not fixed in 
the "out" flow. In Equation (1.18), the emission flow rate is calculated on the basis of both "out" and "down" flow rates. Both flow rates are considered because the purge rate of the harvesting section is based on the "out" flow rate excluding the "down" flow rate. The "product" flow rate is defined in Equation (1.19). Constraints (1.20) and (1.21) set the upper bounds for the total feed flow rate and the makeup flow rate as fu and $m u_{i, j, k}$, respectively. Equation (1.22) enforces the demands of major biofuel products $p d_{k}$ to be strictly satisfied.

Based on the mass balance constraints, we evaluate several costs that contribute to the total investment cost and OPEX. Equations (1.23)-(1.27) model the capital cost $C C_{i, j}$ of technology $j$ in section $i$ using piecewise linear approximation functions.

$$
\begin{aligned}
& \sum_{l \in L}\left(c c i_{i, j, l} \cdot W I_{i, j, l}+c c o_{i, j, l} \cdot W O_{i, j, l}\right)=C C_{i, j}, \quad \forall i \in I, j \in J \\
& \sum_{l \in L}\left(m i_{i, j, l} \cdot W I_{i, j, l}\right)=\sum_{k \in E I_{i, j}} Q I_{i, j, k}, \quad \forall i \in I, j \in J \\
& \sum_{l \in L}\left(m o_{i, j, l} \cdot W O_{i, j, l}\right)=\sum_{k \in K O_{i, j}} Q O_{i, j, k}, \quad \forall i \in I, j \in J \\
& \sum_{l \in L} W I_{i, j, l}=1, \quad \forall i \in I, j \in J \\
& \sum_{l \in L} W O_{i, j, l}=1, \quad \forall i \in I, j \in J
\end{aligned}
$$

In Equations (1.23)-(1.27), $L$ is the set for partition points of the original nonlinear concave power functions [46]. The capital cost data retrieved from literature are associated with either input or output for various technologies. $W I_{i, j, l}$ and $W O_{i, j, l}$ 
are SOS2 (special ordered sets of type 2) variables, which allow at most two consecutive elements over the set $L$ to be non-zero. $m i_{i, j, l}$ and $m o_{i, j, l}$ are the base "in" and "out" flow rates of interested species $k$ in technology $j$ in section $i$, respectively. The sets for the species that are used for economic evaluation are denoted as $K I_{i, j}$ and $K O_{i, j .} . c c i_{i, j, l}$ and $c c o_{i, j, l}$ are the capital costs of technology $j$ in section $i$ with respect to flow rates $m i_{i, j, 1}$ and $m o_{i, j, l}$. The economic evaluation constraints as well as the original definition of ROI are shown in Appendix B.

$$
R O I=\frac{N_{0}}{D}
$$

where

$$
\begin{aligned}
& N_{0}=\sum_{i, j, k} c 1_{k} \cdot M_{i, j, k}^{\text {product }}+\sum_{i, j, k} c 2_{k} \cdot M_{i, j, k}^{\text {makeup }}+\sum_{i, j, k} c 3_{i, j, k} \cdot M_{i, j, k}^{\text {out }} \\
& +\sum_{i, j, k} c 4_{i, j, k} \cdot M_{i, j, k}^{i n}+c 5 \cdot \sum_{i, j, k \in K E} M_{i, j, k}^{e m s s i o n}+c 6 \cdot \sum_{i, j} C C_{i, j}+c 7^{\prime} \\
& D=c 8 \cdot \sum_{i, j} C C_{i, j}+\sum_{j} c 9_{j} \cdot Q O_{1, j, 15} \\
& c l_{k}=(1-t r) \cdot(1-g e c) \cdot h \cdot p p_{k}, \\
& c 2_{k}=-(1-t r) \cdot h \cdot f p_{k}, \\
& c 3_{i, j, k}=-(1-t r) \cdot h \cdot\left(\text { ep } \cdot u p c o_{i, j, k}+h p \cdot u h c o_{i, j, k}+c p \cdot u c c o_{i, j, k}\right), \\
& c 4_{i, j, k}=-(1-t r) \cdot h \cdot\left\{e p \cdot\left[u p c i_{i, j, k}-(1-g e c) \cdot u p g \cdot p e_{i, j, k}\right]+h p \cdot u h c i_{i, j, k}+c p \cdot u c c i_{i, j, k}\right\}, \\
& c 5=-(1-t r) \cdot h \cdot w p,
\end{aligned}
$$


$c 6=-(1-t r) \cdot[m c c+o o c c 2+p t i c+d c c]$,

$c 7=-(1-t r) \cdot(O C+o o c c l)$,

$c 8=(1+w c c) \cdot(1+s c c)$,

$c 9_{j}=(1+w c c) \cdot l p /$ pro $_{j}$.

In order to provide a compact presentation of the objective function, we combine the terms with the same variables in the numerator and the denominator of $R O I$ and introduce parameters $c 1$ to $c 9$ as the coefficients for various variables. Moreover, $N_{o}$ and $D$ denote the net earnings and total investment cost, respectively. Then the deterministic problem becomes:

(DMS) $\quad \max \quad R O I$ as defined in (1.28)

s.t. $\quad$ Network configuration constraints (1.1)-(1.5)

Mass balance constraints (1.7)-(1.22)

Capital cost evaluation constraints (1.23)-(1.27)

\subsubsection{Uncertainty set}

There are many parameters in the deterministic model and all of these parameters demonstrate certain degrees of uncertainty in practice, while market prices, feedstock availability, and product demands are the major factor influencing the profitability of biomass-based network designs [216]. For ease of exposition, we assume the remaining parameters are less volatile and can be precisely predicted and evaluated. We focus on price uncertainty, and supply and demand uncertainty 
as it is widely addressed and well-documented. Specifically, we consider uncertainty in $f p_{k}, p p_{k}, e p, h p, c p, w p, f u, m u_{i, j, k}$, and $p d_{k}$, which are introduced and included in the deterministic model (DMS).

$$
\begin{aligned}
& c 1_{k}=d 1_{k} \cdot \overline{c 1}_{k}, \quad \forall k \in K \\
& c 2_{k}=d 2_{k} \cdot \overline{c 2}_{k}, \quad \forall k \in K \\
& c 31_{i, j, k}=-d 31 \cdot(1-t r) \cdot h \cdot e p \cdot u p c o_{i, j, k}, \quad \forall i \in I, j \in J, k \in K \\
& c 32_{i, j, k}=-d 32 \cdot(1-t r) \cdot h \cdot h p \cdot u h c o_{i, j, k}, \quad \forall i \in I, j \in J, k \in K \\
& c 33_{i, j, k}=-d 33 \cdot(1-t r) \cdot h \cdot c p \cdot u c c o_{i, j, k}, \quad \forall i \in I, j \in J, k \in K \\
& c 41_{i, j, k}=-d 31 \cdot(1-t r) \cdot h \cdot e p \cdot\left[u p c i_{i, j, k}-(1-g e c) \cdot u p g \cdot p e_{i, j, k}\right], \\
& c 42_{i, j, k}=-d 32 \cdot(1-t r) \cdot h \cdot h p \cdot u h c i_{i, j, k}, \quad \forall i \in I, j \in J, k \in K \\
& c 43_{i, j, k}=-d 33 \cdot(1-t r) \cdot h \cdot c p \cdot u c c i_{i, j, k}, \quad \forall i \in I, j \in J, k \in K \\
& c 5=d 5 \cdot \overline{c 5}
\end{aligned}
$$

Coefficients $c 1, c 2, c 3, c 4$, and $c 5$ in the definition of $R O I$ in (1.28) are influenced by the price-related uncertain parameters $f p_{k}, p p_{k}, e p, h p, c p$, and $w p$. The nominal values of these uncertain coefficients are denoted as $\overline{c l}, \overline{c 2}, \overline{c 3}, \overline{c 4}$, and $\overline{c 5}$, respectively. $c 1, c 2, c 31, c 32, c 33, c 41, c 42, c 43$, and $c 5$ represent the maximum deviations from the nominal values due to various uncertain parameters. These maximum deviations are defined by Equations (1.29)-(1.37). $d 1 k, d 2 k, d 31$, 
$d 32, d 33$, and $d 5$ denote the maximum deviation coefficients based on nominal values of $f p k, p p k, e p, h p, c p$, and $w p$, respectively.

$$
\begin{array}{rr}
c 1_{k}=\overline{c 1}_{k}+c 1_{k} \cdot T 1_{k}, \quad \forall k \in K & \\
c 2_{k}=\bar{c}_{k}+c 2_{k} \cdot T 2_{k}, \quad \forall k \in K & \\
c 3_{i, j, k}=\overline{c 3}_{i, j, k}+c 31_{i, j, k} \cdot T 31+c 32_{i, j, k} \cdot T 32+c 33_{i, j, k} \cdot T 33, & \forall i \in I, j \in J, k \in K \\
c 4_{i, j, k}=\overline{c 4}_{i, j, k}+c 41_{i, j, k} \cdot T 31+c 42_{i, j, k} \cdot T 32+c 43_{i, j, k} \cdot T 33, & \forall i \in I, j \in J, k \in K \\
c 5=\overline{c 5}+c 5 \cdot T 5 &
\end{array}
$$

Next, we introduce a set of continuous variables $\left(T 1_{k}, T 2 k, T 31, T 32, T 33\right.$, and $\left.T 5\right)$, each of which is bounded by $[-1,1]$. As shown in Equations (1.38)-(1.42), these variables are used to construct an uncertain region for each uncertain parameter. For instance, $c l_{k}$ can vary within $\left[\overline{c l}_{k}-c l_{k}, \overline{c l}_{k}+c 1_{k}\right]$ with $c l_{k}=\overline{c l}_{k}+c l_{k} \cdot T l_{k}$. Note that both $c 3$ and $c 4$ are affected by $e p, h p$, and $c p$ simultaneously. Specifically, $c 31$ and $c 41$ are associated with $e p, c 32$ and $c 42$ are associated with $h p, c 33$ and $c 43$ are associated with $c p$. Therefore, three variables T31, T32, and T33 are used in both definitions of $c 3$ and $c 4$ corresponding to the levels of variation of $e p, h p$, and $c p$, respectively. There are two reasons for modeling the variations of $c 3$ and $c 4$ using separate variables instead of a lumped variable. First, following the definitions of $c 1, c 2$, and $c 5$, we identify and specify three sources of 
uncertainty for parameters $c 3$ and $c 4$. In contrast, a lumped variation would deny any physical interpretation. Second, the current uncertainty definition is critical for determining the impacts of different uncertain parameters on the optimal objective function value.

$$
\begin{aligned}
& \sum_{k}\left(\left|T 1_{k}\right|+\left|T 2_{k}\right|\right)+|T 31|+|T 32|+|T 33|+|T 5| \leq \Gamma 1 \\
& U 1=\left\{c 1, c 2 \in \mathbb{R}^{|k|}, c 3, c 4 \in \mathbb{R}^{|i||||||| k \mid}, c 5 \in \mathbb{R}:\right. \text { Constraints (3.29)-(3.43), } \\
& \left.T 1_{k}, T 2_{k}, T 31, T 32, T 33, T 5 \in[-1,1], \forall k \in K\right\}
\end{aligned}
$$

Following the approach by Bertsimas and Sim [140], an uncertainty budget $\Gamma 1$ is further introduced in Constraint (1.43) to control the robustness of the optimal solution. If a budget is set to 0 , no uncertainty is allowed for the above parameters, and the corresponding parameters take nominal values. If a budget is set to the maximum number of the uncertain parameters, all the uncertain parameters are allowed to be realized, and the robust optimal solution will be the most conservative. When the budget increases from 0 to the maximum value, the optimal solution becomes more conservative. Note that as opposed to continuous budgets, an integer budget can be interpreted as the number of parameters that are allowed to be uncertain. We focus on integer budgets in this chapter. Based on the previously introduced relationship (1.29)-(1.43), a price uncertainty set $U 1$ is formulated as in Equation (1.44). 


$$
\begin{aligned}
& f u=d 6 \cdot \overline{f u} \\
& m u_{i, j, k}=d 7_{k} \cdot \overline{m u}_{i, j, k}, \quad \forall i \in I, j \in J, k \in K \\
& p d_{k}=d 8_{k} \cdot \overline{p d}_{k}, \quad \forall k \in K P \\
& f u=\overline{f u}+f u \cdot T 6 \\
& m u_{i, j, k}=\overline{m u}_{i, j, k}+m u_{i, j, k} \cdot T 7_{k}, \quad \forall i \in I, j \in J, k \in K \\
& p d_{k}=\overline{p d}_{k}+p d_{k} \cdot T 8_{k}, \quad \forall k \in K P \\
& |T 6|+\sum_{k \in K}\left|T 7_{k}\right|+\sum_{k \in K P}\left|T 8_{k}\right| \leq \Gamma 2 \\
& U 2=\left\{f u \in \mathbb{R}, m u, p d \in \mathbb{R}^{|k|}:\right. \text { Constraints (3.45)-(3.51), } \\
& \left.T 6, T 7_{k}, T 8_{k} \in[-1,1], \forall k \in K\right\}
\end{aligned}
$$

Following the same fashion, we develop a supply and demand uncertainty set U2 as shown in Equation (1.52).

\subsubsection{Two-stage ARMIFP model}

(AROM) $\max \frac{N_{1}}{D}+\min _{c \in U} \max _{M \in O} \frac{N_{2}}{D}$

s.t. first-stage constraints (1.1)-(1.7), (1.23)-(1.27)

where

$$
\begin{aligned}
& N_{1}=c 6 \cdot \sum_{i, j} C C_{i, j}+c 7 \\
& \begin{aligned}
N_{2}= & \sum_{i, j, k} c 1_{k} \cdot M_{i, j, k}^{\text {product }}+\sum_{i, j, k} c 2_{k} \cdot M_{i, j, k}^{\text {makeup }}+\sum_{i, j, k} c 3_{i, j, k} \cdot M_{i, j, k}^{\text {out }} \\
& \quad+\sum_{i, j, k} c 4_{i, j, k} \cdot M_{i, j, k}^{i n}+c 5 \cdot \sum_{i, j, k \in K E} M_{i, j, k}^{\text {emission }}
\end{aligned} \\
& D=c 8 \cdot \sum_{i, j} C C_{i, j}+\sum_{j} c 9_{j} \cdot Q O_{1, j, 15},
\end{aligned}
$$


$c$ represents the uncertainty parameters,

$U$ is defined in (1.44),

$M$ represents the second-stage decisions,

$O=\{$ the feasible region constructed by Constraints (3.8)-(3.22) $\}$.

We propose a two-stage adaptive robust optimization model (AROM) based on the deterministic model and the uncertainty set. In (AROM), the decisions are made sequentially at two stages: (1) a design stage that involves the "here-and-now" decisions before the uncertainties are realized; and (2) an operational stage that involves the "wait-and-see" decisions after the uncertainties are revealed. The firststage decisions include the binary variables $Y_{i, j}$ in the superstructure configuration constraints (1.1)-(1.5), and the technology capacities $Q I_{i, j, k}$ and $Q O_{i, j, k}$, the technology capital costs $C C_{i, j}$, as well as the SOS2 variables $W I_{i, j p p}$ and $W O_{i, j p}$ in the capital cost evaluation constraints (1.23)-(1.27). In contrast, the mass flow rates $M_{i, j, k}^{n}$ for all types of flows in mass balance constraints (1.7)-(1.22) belong to the second-stage variables.

As shown in the general form (AROM), the problem consists of three levels: the first level problem determines first-stage decisions via maximizing the entire objective function; the second level problem determines the worst-case realization of the uncertain parameters via minimizing the part of objective function associated with uncertainty; in the third level maximization problem, second-stage 
decisions are made given fixed first-stage decisions and revealed uncertain parameters.

In order to formulate the objective function in (AROM), we split the deterministic objective function into two fractional terms based on the variables in its numerator. The numerator of the first term $N_{l}$ involves only first-stage decisions, while that of the second term $N_{2}$ involves only second-stage decisions. As a result, the first term is determined before the realization of uncertainty, and the second term should be determined based on the uncertainty realization and the first-stage decisions. The final form of the objective function is shown in (AROM).

\subsection{Solution strategy}

The proposed model (AROM) cannot be solved directly by off-the-shelf optimization solvers due to the structure of the multi-level mixed integer nonlinear programming (MINLP) problem. The combinatorial nature and nonconvexity stemming from the mixed-integer fractional objective function lead to additional computational difficulty. Unfortunately, the existing algorithms for two-stage adaptive optimization problems cannot handle the mixed-integer fractional objective function. To address this computational challenge, we develop a solution strategy that integrates a parametric algorithm with a column-andconstraint generation algorithm. 


\subsubsection{Parametric algorithm}

As mentioned in the last section, the nonlinear term in the problem is the fractional objective function. There are several methods available to efficiently solve a single-stage MIFP problem. A reformulation-linearization method has been proposed to integrate Charnes-Cooper transformation with Glover's linearization $[113,213,217,218]$. This method transforms the original MIFP to an equivalent MILP or MINLP problem with added variables and constraints. Alternatively, a parametric algorithm with various root-finding methods can return the optimal solution by iteratively solving a parametric problem based on the original MIFP [202]. Computational results showed that the parametric algorithm could be more computationally efficient especially when the problem has a large number of variables and constraints [113, 197, 219]. Additionally, it requires much more effort to reformulate the two-stage ARMIFP problem using Charnes-Cooper transformation than to derive a parametric function for the parametric algorithm. In this chapter, we employ the parametric algorithm to handle the fractional objective function.

To apply the parametric algorithm, we first introduce $f(X l)=\min _{c \in U} \max _{M \in O} N_{2}$, where $X 1$ represents the first-stage variables. Since $D$ in the objective function of (AROM) is dependent on only first-stage variables, we have 
$\max \frac{N_{1}}{D}+\min _{c \in U} \max _{M \in O} \frac{N_{2}}{D}=\max _{X I} \frac{N_{l}(X l)+f(X 1)}{D(X 1)}$, which agrees with the MIFP problem for the parametric algorithm [202]. Next, an auxiliary parametric function $F$ with respect to a new parameter $r$. As shown in (LAROM), $F(r)$ is the optimal objective function value of a two-stage adaptive robust MILP problem. The root of equation $F(r)=0$ corresponds to the original optimal solution. Moreover, the function $F(r)$ has been proved to be concave, monotonically decreasing and continuous with respect to $r$. Taking advantage of the bounded subgradients, an inexact Newton's method can be used to find the root of the $F(r)=0$ [202].

(LAROM) $\quad F(r)=\max N_{l}-r \cdot D+\min _{c \in U} \max _{M \in O} N_{2}$

s.t. first-stage constraints (1.1)-(1.7), (1.23)-(1.27)

where the definitions of $N_{1}, N_{2}$, and $D$ are the same with those in (AROM).

\subsubsection{Column-and-constraint generation algorithm}

Two-stage adaptive robust MILP problems, such as $F(r)$, are usually computationally expensive. One prevalent approach approximates the second-stage decisions as explicit functions of uncertain parameters and employs conventional robust optimization solution approaches to obtain the optimal solution [220]. A significant advantage of this method is its computational tractability even with sophisticated approximation functions [221-225]. A different approach involves 
iteratively solving a master problem and a subproblem to achieve the exact optimal solution $[155,226]$. There are two methods following the spirit of this approach. A Benders decomposition-based method generates constraints with the optimal dual information of the subproblem in each iteration [227]. In contrast, a column-andconstraint generation method dynamically generates constraints with second-stage variables in the primal space [228]. It has been proved that the column-andconstraint generation method is more efficient for large-scale two-stage adaptive robust optimization problems [56, 228]. Therefore, we consider the column-andconstraint generation method to handle $F(r)$. We show the formulation of the subproblem and the master problem in the remaining of this subsection.

The subproblem is dedicated to solving the second-stage problem with fixed firststage decisions. In the second-stage problem, absolute value functions are utilized due to the introduction of Constraints (1.43) and (1.51) in the uncertainty sets. To alleviate the computational difficulty, we simplify the definition of the uncertainty set by predetermining the signs of most continuous variables. In order to do so, we first introduce a useful property of the objective function as Lemma 1. It states the third level maximization problem is monotonically increasing with respect to $T 1_{k}$, and monotonically decreasing with respect to $T 2 k, T 32, T 33$, and $T 5$ for any $k \in K$. Given that $T 1_{k}, T 2_{k}, T 32, T 33$, and $T 5$ vary between $[-1,1]$, minimizing the third 
level maximization problem should return non-positive $T 1_{k}$, and non-negative $T 2_{k}$, T32, $T 33$, and $T 5$. As a result, we can restrict the ranges of $T 1_{k}, T 2 k, T 32, T 33$, and $T 5$ to $[0,1]$ and remove the absolute value functions for $T 1_{k}, T 2_{k}, T 32, T 33$, and $T 5$. In addition, we change the definition of $c 1$ to (1.53). The proof of Lemma 1 is given in Appendix C.

The only exception comes with $T 31$, since $c 41$ can be either positive or negative for various technologies. To handle this term specifically, we introduce two Constraints (1.54) and (1.55), which are equivalent to Constraint (1.43) with the absolute value term. Finally, the simplified uncertainty set is shown as U1s.

$$
\begin{aligned}
& c 1_{k}=\overline{c 1}_{k}-c 1_{k} \cdot T 1_{k}, \quad \forall k \in K \\
& \sum_{k}\left(T 1_{k}+T 2_{k}\right)+T 31+T 32+T 33+T 5 \leq \Gamma 1 \\
& \sum_{k}\left(T 1_{k}+T 2_{k}\right)-T 31+T 32+T 33+T 5 \leq \Gamma 1 \\
& \begin{aligned}
U 1_{S}=\left\{c 1, c 2 \in \mathbb{R}^{|k|}, c 3, c 4 \in \mathbb{R}^{|i||| j|x| k \mid}, c 5 \in \mathbb{R}:\right. \\
\quad \text { Constraints }(3.29)-(3.37),(3.39)-(3.42),(3.53)-(3.55), \\
\left.\quad T 1_{k}, T 2_{k}, T 32, T 33, T 5 \in[0,1], \forall k \in K, T 31 \in[-1,1]\right\}
\end{aligned}
\end{aligned}
$$

The second-stage min-max problem is reformulated by dualizing the inner maximization problem and incorporating the resulting minimization problem into the outer minimization problem. The reformulated problem is a nonlinear programming problem with nonlinearity only in the bilinear terms in the objective 
function. The bilinear terms are products of dual variables and level of deviation variables, including $P 13 \cdot T 6, P 14_{i, j, k} \cdot T 7_{k}$, and $P 15_{k} \cdot T 8_{k}, \forall i \in I, j \in J, k \in K$. Since we consider integer budgets of uncertainty, continuous variable $T 6$ and $T 7_{k}$ can be reduced to binary variables and the related bilinear terms take non-positive values given that both $P 13$ and $P 14_{i, j, k}$ are non-negative variables $[56,229,230]$. As a result, we apply the Glover's linearization scheme for the simplified bilinear terms as in Constraints (1.74)-(1.79) [217]. The last bilinear term is more difficult to simplify since $P 15_{k}$ is a free variable. However, in the minimization problem, we can reformulate $P 15_{k} \cdot T 8_{k}$ to be $-\left|P 15_{k}\right| \cdot T 8_{k}$ without hurting the optimal objective function value. Next, we introduce Constraints (1.80)-(1.83) and replace $\left|P 15_{k}\right|$ with a non-negative variable $U_{k}$. The new bilinear term $U_{k} \cdot T 8_{k}$ can then be simplified using the same approach for the other bilinear terms as in Constraints (1.84)-(1.86). The subproblem formulation is given by (1.57)-(1.88).

(SUB)

$$
\begin{array}{ll}
\min & \sum_{i, j, k}\left(P 1_{i, j, k} \cdot Q O_{i, j, k}+P 2_{i, j, k} \cdot Q I_{i, j, k}\right)+P 13 \cdot \overline{f u}-f u \cdot M 1 \\
& +\sum_{i, j, k} P 14_{i, j, k} \cdot \overline{m u}_{i, j, k}-\sum_{i, j, k} m u_{i, j, k} \cdot M 2_{i, j, k}+\sum_{k} P 15_{k} \cdot \overline{p d}_{k}-\sum_{k} p d_{k} \cdot M 3_{k}
\end{array}
$$




$$
\begin{aligned}
& P 5_{i, k}-P 7_{i, j, k} \geq 0, \quad \forall i \in I, j \in J, k \in K \\
& -P 7_{i, j, k}+P 14_{i, j, k} \geq \overline{c 2}_{k}+c 2_{k} \cdot T 2_{k}, \quad \forall i \in I, j \in J, k \in K \\
& P 2_{i, j, k}+P 6_{i, j, k}-\sum_{k^{\prime}} P 6_{i, j, k^{\prime}} \cdot p_{i, j, k^{\prime}, k}^{i n}+P 7_{i, j, k}-P 8_{i, j, k}-\sum_{k^{\prime}} P 8_{i, j, k^{\prime}} \cdot s c_{i, j, k^{\prime}, k} \cdot x_{i, j} \\
& \geq \overline{c 4}_{i, j, k}+c 41_{i, j, k} \cdot T 31+c 42_{i, j, k} \cdot T 32+c 43_{i, j, k} \cdot T 33, \\
& \forall i \in I, j \in J, k \in K \\
& P 1_{i, j, k}+P 8_{i, j, k}+P 9_{i, j, k}-\sum_{k^{\prime}} P 10_{i, j, k^{\prime}} \cdot s f_{i, j, k^{\prime}, k}^{\text {down }}-P 11_{i, j, k} \cdot s f_{i, j, k}^{e m i s s i o n l} \\
& -P 12_{i, j, k} \cdot s f_{i, j, k}^{\text {product }} \geq \overline{c 3}_{i, j, k}+c 31_{i, j, k} \cdot T 31+c 32_{i, j, k} \cdot T 32+c 33_{i, j, k} \cdot T 33, \\
& \forall i \in I, j \in J, k \in K \\
& -P 4_{i+1, k} \cdot p_{i+1}^{\text {down }}-P 9_{i, j, k}+P 10_{i, j, k}+P 11_{i, j, k} \cdot s f_{i, j, k}^{\text {emission } 2} \geq 0 \\
& \forall 1 \leq i \leq 7, j \in J, k \in K \\
& -P 9_{8, j, k}+P 10_{8, j, k}+P 11_{8, j, k} \cdot s f_{8, j, k}^{e m i s s i o n 2} \geq 0, \quad \forall j \in J, k \in K \\
& -\sum_{i^{\prime} \in I_{i}^{\text {recycle }}} P 5_{i^{\prime}, k}-P 9_{i, j, k} \geq 0, \quad \forall i \in I, j \in J, k \in K \\
& -P 9_{i, j, k}+P 11_{i, j, k} \geq \overline{c 5}+c 5 \cdot T 5, \quad \forall i \in I, j \in J, k \in K E \\
& -P 9_{i, j, k}+P 11_{i, j, k} \geq 0, \quad \forall i \in I, j \in J, k \notin K E \\
& -P 9_{i, j, k}+P 12_{i, j, k}+P 15_{k} \geq \overline{c 1}_{k}-c 1_{k} \cdot T 1_{k}, \quad \forall i \in I, j \in J, k \in K P \\
& -P 9_{i, j, k}+P 12_{i, j, k} \geq \overline{c 1}{ }_{k}-c 1_{k} \cdot T 1_{k}, \quad \forall i \in I, j \in J, k \notin K P \\
& \sum_{k}\left(T 1_{k}+T 2_{k}\right)+T 31+T 32+T 33+T 5 \leq \Gamma 1 \\
& \sum_{k}\left(T 1_{k}+T 2_{k}\right)-T 31+T 32+T 33+T 5 \leq \Gamma 1 \\
& M 1 \leq P 13 \\
& 0 \leq M 1 \leq T 6 \cdot u 1
\end{aligned}
$$




$$
\begin{aligned}
& M 1 \geq P 13-(1-T 6) \cdot u 1 \\
& M 2_{i, j, k} \leq P 14_{i, j, k}, \quad \forall i \in I, j \in J, k \in K \\
& 0 \leq M 2_{i, j, k} \leq T 7_{k} \cdot u 2, \quad \forall i \in I, j \in J, k \in K \\
& M 2_{i, j, k} \geq P 14_{i, j, k}-\left(1-T 7_{k}\right) \cdot u 2, \quad \forall i \in I, j \in J, k \in K \\
& P 15_{k}+u 3 \cdot \omega_{k} \geq U_{k}, \quad \forall k \in K P \\
& -P 15_{k}+u 3 \cdot\left(1-\omega_{k}\right) \geq U_{k}, \quad \forall k \in K P \\
& P 15_{k} \leq U_{k}, \quad \forall k \in K P \\
& -P 15_{k} \leq U_{k}, \quad \forall k \in K P \\
& M 3_{k} \leq U_{k}, \quad \forall k \in K P \\
& 0 \leq M 3_{k} \leq T 8_{k} \cdot u 3, \quad \forall k \in K P \\
& M 3_{k} \geq U_{k}-\left(1-T 8_{k}\right) \cdot u 3, \quad \forall k \in K P \\
& T 6+\sum_{k \in K} T 7_{k}+\sum_{k \in K P} T 8_{k} \leq \Gamma 2 \\
& T 1_{k}, T 2_{k}, T 32, T 33, T 5 \in[0,1], T 31 \in[-1,1], \omega_{k}, T 6, T 7_{k}, T 8_{k} \in\{0,1\}, \\
& P 1_{i, j, k}, P 2_{i, j, k}, P 13, P 14_{i, j, k} \geq 0, \forall i, j, k
\end{aligned}
$$

The master problem possesses both the first-stage and second-stage constraints but with a subset of uncertainty realizations. As a result, the master problem is equipped with a relaxed feasible region, resulting in an upper bound of the optimal objective function value. To formulate the master problem, we replace the secondstage objective function with a new variable $\eta$, which is constrained by (1.91). Next, we introduce several new constraints (1.92)-(1.106) based on the original 
second-stage constraints and plug them into the original first-stage problem. Moreover, every second-stage variable is assigned with a new index $s$, which denotes identified uncertainty realizations.

(MA) $\max c 6 \cdot \sum_{i, j} C C_{i, j}+c 7-r \cdot\left(c 8 \cdot \sum_{i, j} C C_{i, j}+\sum_{j} c 9_{j} \cdot Q O_{l, j, l 5}\right)+\eta$

s.t. Constraints (1)-(7), (23)-(27)

$\eta \leq \sum_{i, j, k} c 1_{k, s} \cdot M_{i, j, k, s}^{\text {product }}+\sum_{i, j, k} c 2_{k, s} \cdot M_{i, j, k, s}^{\text {makeup }}$

$$
+\sum_{i, j, k} c 3_{i, j, k, s} \cdot M_{i, j, k, s}^{\text {out }}+\sum_{i, j, k} c 4_{i, j, k, s} \cdot M_{i, j, k, s}^{i n}+c 5_{s} \cdot \sum_{i, j, k} M_{i, j, k, s}^{\text {emission }}, \quad \forall s \in S
$$

$M_{i, j, k, s}^{\text {out }} \leq Q O_{i, j, k}, \quad \forall i \in I, j \in J, k \in K, s \in S$

$M_{i, j, k, s}^{i n} \leq Q I_{i, j, k}, \quad \forall i \in I, j \in J, k \in K, s \in S$

$\sum_{j \in J} M_{l, j, k, s}^{u p}-f_{k}^{\text {feed }} \cdot M_{s}^{\text {feed }}=0, \quad \forall k \in K, s \in S$

$\sum_{j \in J} M_{i, j, k, s}^{u p}-p_{i}^{\text {down }} \cdot \sum_{i^{\prime} \in i_{i}^{u p}, j \in J} M_{i^{\prime}, j, k, s}^{\text {down }}=0, \quad \forall 2 \leq i \leq 8, k \in K, s \in S$

$\sum_{j \in J} M_{i, j, k, s}^{\text {reuse }}-\sum_{i^{\prime} \in I_{i}^{\text {erse e }}, j \in J} M_{i^{\prime}, j, k, s}^{\text {recyle }}=0, \quad \forall i \in I, k \in K, s \in S$

$M_{i, j, k, s}^{i n}-\sum_{k^{\prime} \in K} p_{i, j, k, k^{\prime}}^{i n} \cdot M_{i, j, k^{\prime}, s}^{i n}=0, \quad \forall i \in I, j \in J, k \in K, s \in S$

$M_{i, j, k, s}^{\text {in }}-M_{i, j, k, s}^{u p}-M_{i, j, k, s}^{\text {reuse }}-M_{i, j, k, s}^{\text {makeup }}=0, \quad \forall i \in I, j \in J, k \in K, s \in S$

$M_{i, j, k, s}^{\text {out }}-M_{i, j, k, s}^{i n}-\sum_{k^{\prime} \in K}\left(s c_{i, j, k, k^{\prime}} \cdot x_{i, j} \cdot M_{i, j, k^{\prime}, s}^{i n}\right)=0, \forall i \in I, j \in J, k \in K, s \in S$

$M_{i, j, k, s}^{\text {out }}-M_{i, j, k, s}^{\text {down }}-M_{i, j, k, s}^{\text {recycle }}-M_{i, j, k, s}^{\text {emission }}-M_{i, j, k, s}^{\text {product }}=0$,

$$
\forall i \in I, j \in J, k \in K, s \in S
$$




$$
\begin{aligned}
& M_{i, j, k, s}^{\text {down }}-\sum_{k^{\prime} \in K}\left(s f_{i, j, k, k^{\prime}}^{\text {down }} \cdot M_{i, j, k^{\prime}, s}^{\text {out }}\right)=0, \quad \forall i \in I, j \in J, k \in K, s \in S \\
& \begin{array}{c}
M_{i, j, k, s}^{\text {emission }}-s f_{i, j, k}^{\text {emissionl }} \cdot M_{i, j, k, s}^{\text {out }}+s f_{i, j, k}^{\text {emission2 }} M_{i, j, k, s}^{\text {product }}=0, \\
\forall i \in I, j \in J, k \in K, s \in S
\end{array} \\
& M_{i, j, k, s}^{\text {product }}-s f_{i, j, k}^{\text {product }} \cdot M_{i, j, k, s}^{\text {out }}=0, \quad \forall i \in I, j \in J, k \in K, s \in S \\
& M_{s}^{\text {feed }} \leq f u_{s}, \quad \forall s \in S \\
& M_{i, j, k, s}^{\text {makeup }} \leq m u_{i, j, k, s}, \quad \forall i \in I, j \in J, k \in K, s \in S \\
& \sum_{i \in I, j \in J} M_{i, j, k, s}^{\text {product }}=p d_{k, s}, \quad \forall k \in K P, s \in S
\end{aligned}
$$

\subsubsection{Optimization solution methods}

In order to effectively solve the two-stage ARMIFP, we integrate the parametric algorithm with the column-and-constraint generation algorithm. As shown in Figure 18, the proposed algorithm employs the parametric algorithm in the outer loop, while the column-and-constraint generation algorithm is utilized in the inner loop to handle the auxiliary two-stage adaptive robust MILP problem. The entire algorithm starts by setting $r=0$, upper bound $u b$ to be a sufficiently large number, and solving the deterministic problem (DMS) to obtain the initial firststage decisions. In addition, iteration count for the outer loop iter are initialized before the outer loop begins.

Proposed solution algorithm 1: $\quad r \leftarrow 0, u b \leftarrow+\infty$, iter $\leftarrow 0$; 
2: Solve (DM) to obtain the optimal first-stage decisions $C C^{*}$ and $Q O^{*}$;

3: $\quad c c f_{i, j} \leftarrow C C_{i, j}^{*}, \quad q o f_{i, j, k} \leftarrow Q O_{i, j, k}^{*}, \quad \forall i, j, k$;

4: while $|u b o| \geq \varepsilon$ do

5: $\quad$ iter $\leftarrow($ iter +1$), l b \leftarrow 0$, gap $\leftarrow+\infty, s \leftarrow 0, S \leftarrow \varnothing ;$

6: $\quad$ while gap $\geq \varepsilon$ do

7: $\quad s \leftarrow(s+1)$

8: $\quad$ Solve (SUB) to obtain $s o^{*}, T 1_{k}{ }^{*}, T 2_{k}{ }^{*}, T 31^{*}, T 32^{*}, T 33^{*}, T 5^{*}, T 6^{*}, T 7_{k}^{*}, T 8_{k}^{*}$, $U_{k}{ }^{*}$, and $P 15_{k}{ }^{*}$;

9:

10:

11:

12:

13:

14:

15:

16:

17:

18:

19:

20:

21:

22: end $l b=\max \left\{l b, c 6 \cdot \sum_{i, j} c c f_{i, j}+c 7-r \cdot\left(c 8 \cdot \sum_{i, j} c c f_{i, j}+\sum_{j} c 9_{j} \cdot q o f_{i, j, 15}\right)+s o^{*}\right\}$

$$
\begin{aligned}
& S \leftarrow S \bigcup\{s\} \\
& c 1_{k, s} \leftarrow\left(\overline{c 1}_{k}-c 1_{k} \cdot T 1_{k}^{*}\right), c 2_{k, s} \leftarrow\left(\overline{c 2}_{k}+c 2_{k} \cdot T 2_{k}^{*}\right), ; c 5_{s} \leftarrow\left(\overline{c 5}+c 5 \cdot T 5^{*}\right)
\end{aligned}
$$$$
c 3_{i, j, k, s} \leftarrow\left(\bar{c}_{i, j, k}+c 31_{i, j, k} \cdot T 31^{*}+c 32_{i, j, k} \cdot T 32^{*}+c 33_{i, j, k} \cdot T 33^{*}\right), \forall i, j, k
$$$$
c 4_{i, j, k, s} \leftarrow\left(\overline{c 4}_{i, j, k}+c 41_{i, j, k} \cdot T 31^{*}+c 42_{i, j, k} \cdot T 32^{*}+c 43_{i, j, k} \cdot T 33^{*}\right), \forall i, j, k
$$

$$
\begin{aligned}
& f u_{s} \leftarrow\left(\overline{f u}-f u \cdot T 6^{*}\right) ; m u_{i, j, k, s} \leftarrow\left(\overline{m u}_{i, j, k}-m u_{i, j, k} \cdot T 7_{k}^{*}\right), \quad \forall i, j, k \\
& p d_{k, s} \leftarrow\left(\overline{p d}_{k}-U_{k}^{*} / P 15_{k}^{*} \cdot p d_{k} \cdot T 8_{k}^{*}\right), \quad \forall k \in K P
\end{aligned}
$$

Create second-stage variables with respect to index $s$; Solve (MA) to obtain $m o^{*}, \eta^{*}, C C^{*}$ and $Q O^{*}$;

23: return $u b$;

$$
u b \leftarrow \min \left\{u b, m o^{*}\right\}, g a p \leftarrow(u b-l b)
$$$$
c c f_{i, j} \leftarrow C C_{i, j}^{*}, q o f_{i, j, k} \leftarrow Q O_{i, j, k}^{*}, \quad \forall i, j, k
$$

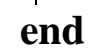

Figure 18. Pseudo code of the proposed algorithm.

Before the algorithm enters the inner loop, lower bound $1 b$, gap gap, iteration count for the inner loop $s$, and the set of the uncertain parameters $S$ are specified.

Next, the inner loop initiates by solving the subproblem (SUB). As introduced in 
the previous subsection, the optimal objective function value $s o^{\circ}$ can used to update the lower bound as shown in line 9 of Figure 18. The current iteration is added into the set of identified uncertain parameters. As the optimal solution of (SUB) is in the dual space, we evaluate the identified uncertain parameters using the equations in line 11-15 of Figure 18. A set of second-stage variables with respect to the identified uncertain parameters are added to the master problem (MA). The algorithm then solves the master problem with all identified uncertain parameters. Subsequently, the optimal objective function value $m o^{*}$ of the master problem is used to update the current upper bound, taken as the larger value between the existing upper bound and mo* Later, the inner loop checks the absolute difference between lower and upper bound. The inner loop terminates if the gap is sufficiently close to the tolerance. Otherwise, the optimal first-stage decisions are passed to a new subproblem, and the interaction between the subproblem and master problem continue until the stopping criterion is satisfied.

In the outer loop of the proposed algorithm, the optimal objective function value or the current upper bound from the inner loop is compared with a predefined tolerance. The outer loop terminates if the current upper bound from the inner loop is smaller than the tolerance, indicating the optimal objective function value of the auxiliary robust optimization problem is sufficiently close to zero. 
Otherwise, a new $r$ is defined following the formula in line 21 of Figure 18. The new $r$ is then used to obtain a new upper bound from the inner loop and the outer loop keeps iterating until an upper bound within the tolerance is found.

\subsection{Results and discussion}

Table 5. Major uncertain parameters in the model.

\begin{tabular}{|c|c|c|c|c|c|c|c|}
\hline Species & $\begin{array}{c}\text { Ava./ } \\
\text { Deman } \\
d(t / h)\end{array}$ & $\begin{array}{l}\text { Price } \\
(\$ / \mathrm{t})\end{array}$ & $\begin{array}{c}\text { Maximum } \\
\text { deviation } \\
\text { coefficien } \\
t\end{array}$ & Species & $\begin{array}{l}\text { Ava./ } \\
\text { Deman } \\
d(t / h)\end{array}$ & Price $(\$ / t)$ & $\begin{array}{c}\text { Maximum } \\
\text { deviation } \\
\text { coefficien } \\
\mathrm{t} \\
\end{array}$ \\
\hline Feed gas & 6000 & N/A & $10 \%$ & $\begin{array}{l}\text { Hydrochl } \\
\text { oric acid }\end{array}$ & 4 & 200 & $10 \%$ \\
\hline Urea & 50 & 270 & $10 \%$ & Lipase & 2.5 & 30,000 & $10 \%$ \\
\hline DAP & 50 & 460 & $10 \%$ & Hydrogen & 10 & 3,000 & $10 \%$ \\
\hline $\begin{array}{l}\text { Polyelectrol } \\
\text { yte }\end{array}$ & 2 & $\begin{array}{c}2,50 \\
0\end{array}$ & $10 \%$ & $\begin{array}{l}\text { Isobutene } \\
\text { /Isobutane }\end{array}$ & 2.5 & 1,500 & $10 \%$ \\
\hline $\begin{array}{l}\text { Sodium } \\
\text { hydroxide }\end{array}$ & 5 & 328 & $10 \%$ & $\begin{array}{c}\text { Diethyl } \\
\text { succinate }\end{array}$ & 0.2 & 3,500 & $10 \%$ \\
\hline $\begin{array}{l}\text { Poly- } \\
\text { aluminum } \\
\text { chloride }\end{array}$ & 1 & 929 & $10 \%$ & Fertilizer & N/A & 100 & N/A \\
\hline $\begin{array}{l}\text { Aluminum } \\
\text { sulfate }\end{array}$ & 3.5 & $\begin{array}{c}1,52 \\
0\end{array}$ & $10 \%$ & $\begin{array}{c}\text { Propylene } \\
\text { glycol }\end{array}$ & N/A & 1,600 & N/A \\
\hline Chitosan & 2 & $\begin{array}{c}1,00 \\
0\end{array}$ & $10 \%$ & $\begin{array}{l}\mathrm{Di}^{-} \\
\text {ether/Tri- } \\
\text { ether }\end{array}$ & N/A & 2,000 & N/A \\
\hline $\begin{array}{l}\text { Poly-y- } \\
\text { glutamic } \\
\text { acid }\end{array}$ & 0.5 & $\begin{array}{c}5,00 \\
0\end{array}$ & $10 \%$ & PHB & N/A & 9,700 & N/A \\
\hline Hexane & 5.5 & 717 & $10 \%$ & Biodiesel & 25 & 1,008 & $10 \%$ \\
\hline Butanol & 6.5 & $\begin{array}{c}1,50 \\
0\end{array}$ & $10 \%$ & $\begin{array}{l}\text { Waste } \\
\text { treatment }\end{array}$ & N/A & 250 & $10 \%$ \\
\hline Methanol & 13 & 500 & $10 \%$ & Electricity & N/A & $\begin{array}{c}70.3 \\
\text { (\$/MWh) }\end{array}$ & $6 \%$ \\
\hline $\begin{array}{l}\text { Sodium } \\
\text { hydroxide }\end{array}$ & 8 & 300 & $10 \%$ & Heating & N/A & $\begin{array}{c}29 \\
(\$ / M W h)\end{array}$ & $10 \%$ \\
\hline $\begin{array}{c}\text { Sodium } \\
\text { methoxide }\end{array}$ & 1.2 & $\begin{array}{c}1,50 \\
0 \\
\end{array}$ & $10 \%$ & Cooling & N/A & $\begin{array}{c}14 \\
\text { (\$/MWh) }\end{array}$ & $10 \%$ \\
\hline
\end{tabular}


All computational experiments are performed on a desktop PC with an Intel(R) Core(TM) i5-2400 3.10GHz CPU and 8GB RAM. All of the models and solution algorithms are coded in GAMS 24.4.6 [203]. The subproblem and relaxed master problem are solved using CPLEX 12. The relative optimality tolerance of CPLEX 12 is set to $10^{-6}$, and the absolute optimality tolerance for the proposed algorithm is set to $10^{-6}$. The deterministic problem has 37,854 continuous variables, 48 discrete variables, and 63,920 constraints; the master problem has 46,003 continuous variables, 48 discrete variables, and 39,589 constraints; the subproblem has 43,522 continuous variables, 105 discrete variables, and 48,982 constraints. Most parameters are taken from previous studies [21, 22], and the uncertain data are evaluated based on historical records [212, 231-235]. The uncertainty information including the feedstock availability, biofuel demand, and prices are shown in Table 5. 


\subsubsection{Maximizing return on investment}

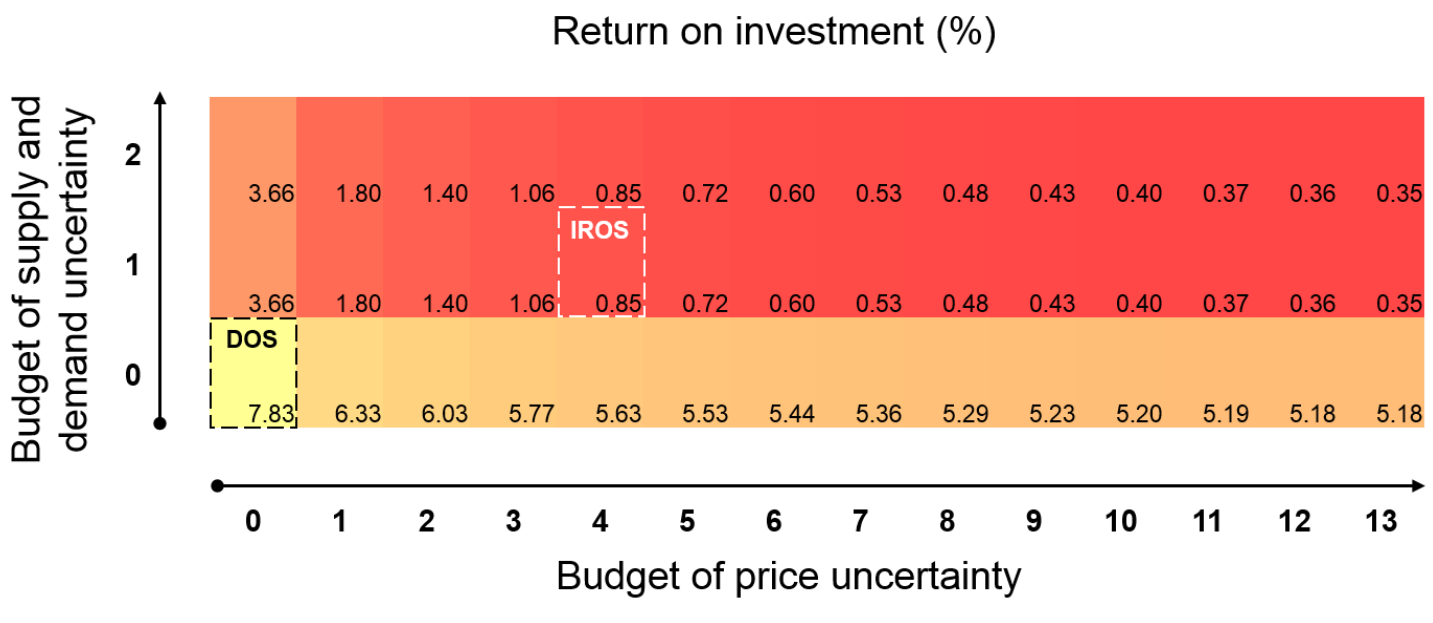

7.83

Figure 19. A heat map for the robust return on investment (\%) under various budgets of uncertainty. DOS is the deterministic optimal solution, and IROS is the illustrative robust optimal solution.

A budget of uncertainty is a significant parameter to control the robustness of the optimal solutions of the proposed two-stage ARMIFP problem. When the budget of uncertainty increases, more deviations of the uncertain parameters from corresponding nominal values are allowed. Subsequently, there can be more uncertainty realizations and the robust optimal solution, or the worst-case optimal solution in all uncertainty realizations, usually becomes worse. By varying the budget of uncertainty, we are able to obtain a set of robust optimal solutions which reveal the tradeoff between risk and ROI. It can be clearly seen in Figure 19 that as the budgets of uncertainty increase, the robust optimal ROI decreases. The most 
conservative ROI of $0.35 \%$ is obtained when the budget of price uncertainty is 13 and the budget of supply and demand uncertainty is 1 . When we hold either of the budgets and increase the other, no smaller ROI could be obtained if the budget exceeds that of the most conservative ROI. This is because either the involved uncertain parameters have reached the maximum amount of deviation for the chosen processing pathway, or the remaining uncertain parameters do not influence the optimal objective function value.

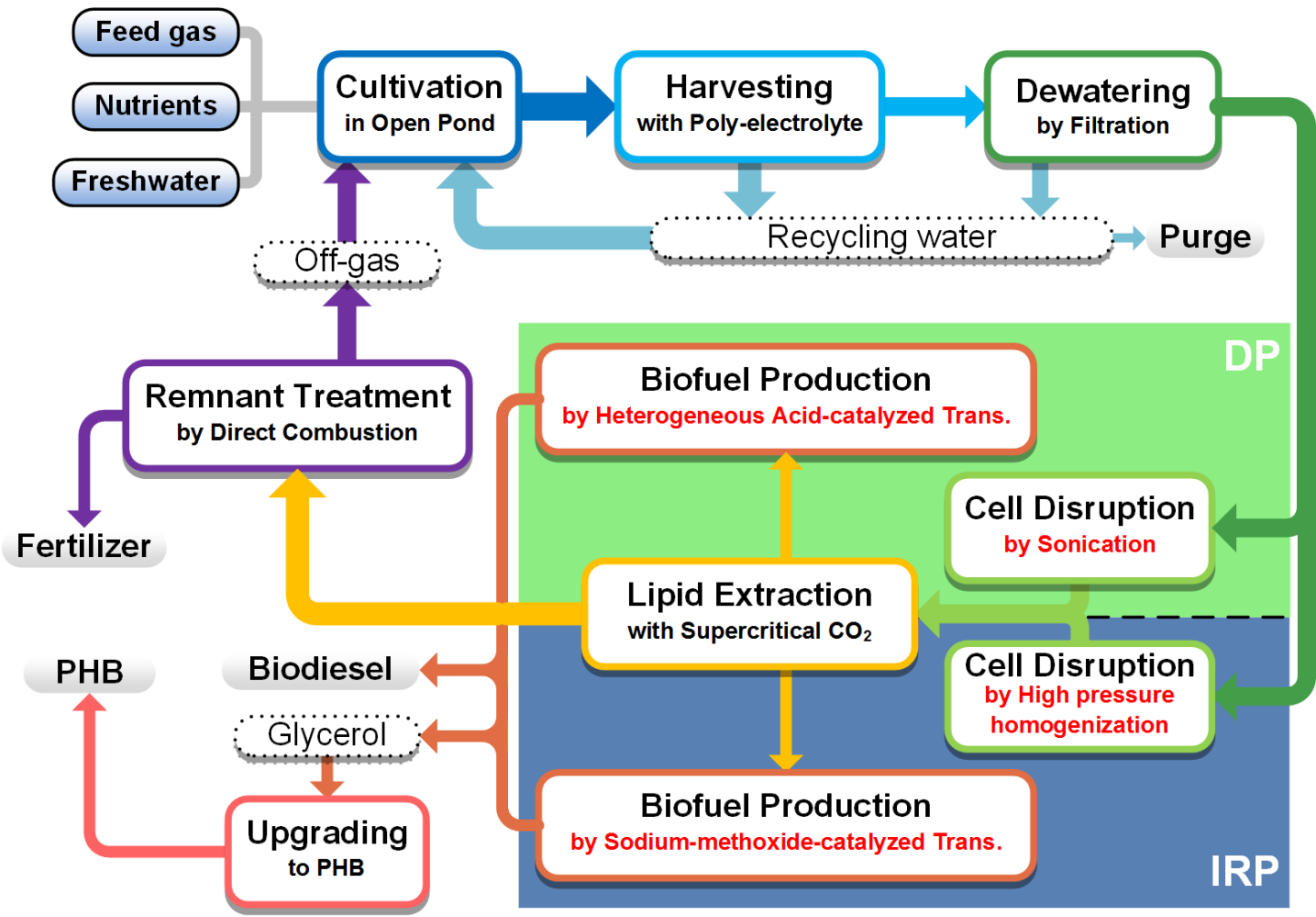

Figure 20. Three identified optimal processing pathways. DP is the deterministic optimal pathway; IRP is the illustrative robust optimal pathway. 
We consider two optimal solutions in Figure 19 to demonstrate the advantages of robust optimal solutions. The point corresponding to 0 budget for both price uncertainty and supply and demand uncertainty is referred to as the deterministic optimal solution (DOS). As all risks are ignored, the DOS shows the most economically favorable performance compared with the other robust optimal solutions. As shown in Figure 20, the DOS selects open pond, poly-electrolytebased flocculation, filtration, sonication, supercritical $\mathrm{CO}_{2}$ extraction, heterogeneous acid-catalyzed transesterification, PHB production, and direct combustion for biogas utilization. In contrast, the illustrative robust optimal solution (IROS) corresponds to a price uncertainty budget of 4 and a supply and demand uncertainty budget of 1 . Larger uncertainty budgets lead to more conservative economic performance. This robust optimal solution selects high pressure homogenization in the cell disruption section and sodium-methoxidecatalyzed transesterification in the biofuel production section.

Table 6. Key optimal results of the deterministic optimal solution (DOS) and the illustrative robust optimal solution (IROS).

\begin{tabular}{ccc}
\hline & DOS & IROS \\
\hline $\begin{array}{c}\text { Budget of price uncertainty } \\
\text { Budget of Supply and } \\
\text { demand uncertainty }\end{array}$ & 0 & 4 \\
Cell disruption technology & 0 & 1 \\
Transesterification & Sonication & $\begin{array}{c}\text { High pressure } \\
\text { homogenization } \\
\text { Sodium-methoxide- } \\
\text { catalyzed }\end{array}$ \\
& 106 &
\end{tabular}


Algae production, $\mathrm{t} / \mathrm{h}$

Biodiesel production, $\mathrm{t} / \mathrm{h}$

Biodiesel production,

$\mathrm{Mgal} /$ year

PHB production, $\mathrm{t} / \mathrm{h}$

Productivity, \%

ROI, \%
176.93

25.00

66.04

0.45

14.13

7.83
86.73

22.50

59.44

0.43

25.94

0.85

In order to understand why different technologies are selected between the two pathways, we provide the key optimal results in Table 6. The DOS shows a lower biomass conversion efficiency, while achieves a higher ROI (highest in Figure 24). The IROS reduces the biomass consumption, but still meets the same biodiesel demand. The major difference between the two solutions is that the IROS is hedged against uncertainty in price, biofuel demand, and feedstock availability. Regarding the objective function value of the IROS, the worst-case realization in the supply and demand uncertainty is identified as a decline in biodiesel demand. However, an increase in biodiesel demand is even more critical because this realization can lead to feasibility issues of economically favorable processing pathways. Specifically, a positive deviation results in a biodiesel demand of $27.5 \mathrm{t} / \mathrm{h}$ (72.64 Mgal/y), which is an infeasible production rate for a processing pathway equipped with sonication in the cell disruption section and heterogeneous acidcatalyzed transesterification in biofuel production. As a result, high pressure homogenization and sodium-methoxide-catalyzed transesterification are selected 
in the robust processing pathway because of a higher biomass utilization efficiency. However, a high biomass utilization efficiency or the robustness in the resulting processing pathways comes at a high operating cost. Overall, the IROS is a much more reliable processing pathway if we take uncertainty into account.

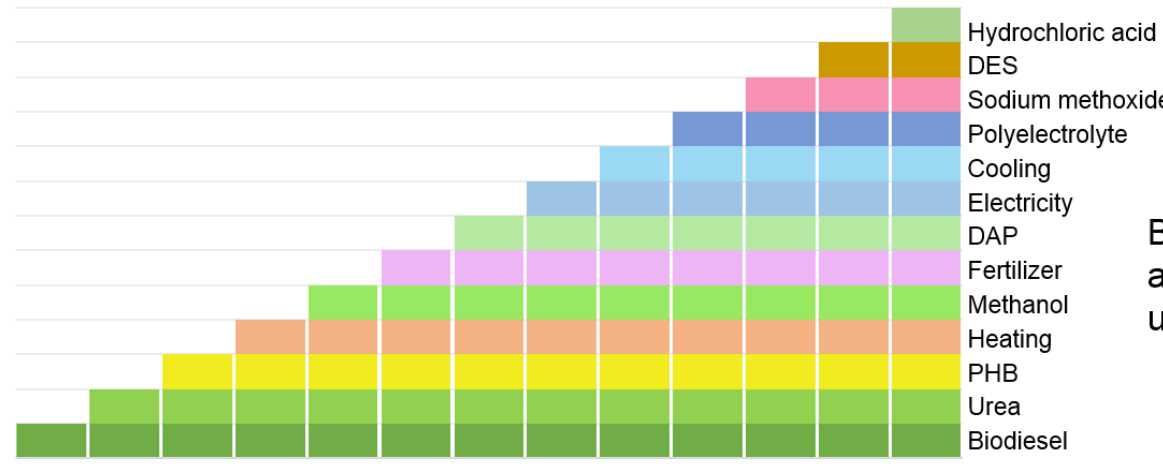

Budget of supply and demand uncertainty $=0$
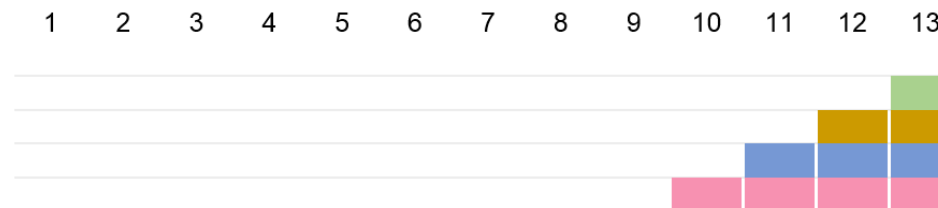

Hydrochloric acid DES Polyelectrolyte Sodium methoxide DAP Fertilizer Cooling Methanol Heating Urea PHB Electricity Biodiesel

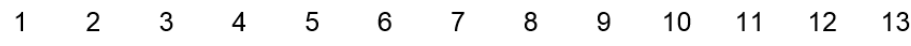

Budget of price uncertainty

Figure 21. The worst-case uncertainty realizations for the robust optimal solutions with varying budgets of uncertainty.

More insights can be obtained if we investigate the distribution of the budgets of uncertainty in the resulting robust optimal solutions. Among various uncertainty 
realizations, we are interested in the worst-case uncertainty realization that contributes to the robust optimal solution. When we collect the worst-case uncertainty realization for each robust optimal solution, we find biofuel demand shows much more impact on the ROI than the other uncertain parameters when the budget of supply and demand uncertainty increases. If the biofuel demand is fixed, the variation in feedstock availability would not affect the optimal ROI. However, as we analyzed previously, if the biofuel demand fluctuates, the available feedstock supply may not be sufficient for certain processing pathways to generate adequate biofuel. We further find that for a fixed budget of supply and demand uncertainty (being 0 or 1), the robust optimal solutions select the same processing pathway. As a result, when we increase the budget of price uncertainty by 1 , the previously selected uncertain prices are always selected again and a new uncertain price is also selected. We show the distribution in Figure 21, where one block represents an uncertain price and the newly selected uncertain price is placed on the top of each column.

In order to understand Figure 21, we start from the fact that when we fix the budget of supply and demand uncertainty, the impacts of all uncertain prices on the objective function are different. Consequently, there must be a ranking of the uncertainty prices based on the degree of decline in the optimal value of ROI when 
an uncertain price is considered. The key of robust optimization is to search for the worst-case realization in all possible realizations. When the budget of price uncertainty is set as 1 , the program outputs the first uncertain price in the ranking because the introduction of this uncertainty results in the worst-case optimal objective function value. When the budget of price uncertainty is set as 2 , the top 2 uncertain prices must be selected. We construct this ranking in Figure 21 following exactly the same procedure. Therefore, the sequence of uncertain prices on the right of each figure reveals the impacts of uncertain prices, being the lower, the more influential. For example, biodiesel price is identified as the most influential uncertain price among those considered in the model, and a decline in biodiesel price leads to the largest decrease in ROI among all uncertain parameters. 


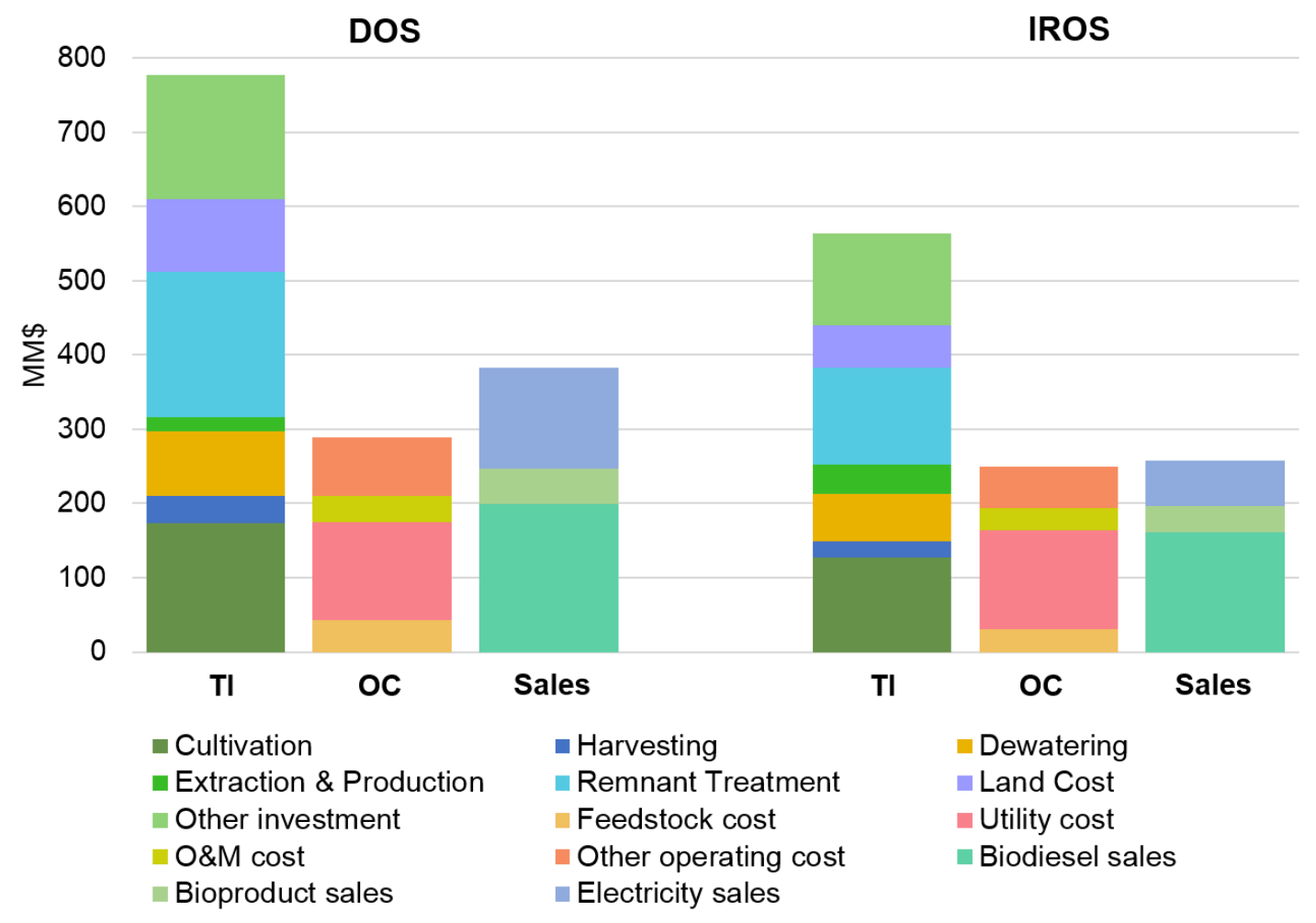

Figure 22. Distribution of the total investment (TI), operating costs (OC), and sales for the deterministic optimal solution (DOS) and the illustrative robust optimal solutions (IROS).

The breakdown of the total investment costs, the operating costs, and the sales for the deterministic optimal solution, and two robust optimal solutions is shown in Figure 22. With a higher biomass utilization efficiency, the total investment cost, the operating cost, and the sales of the IROS are smaller than those of the DOS. However, as the operating cost of the IROS does not decrease as significantly as the total investment cost and the sales, the overall economic performance of the IROS is worse than that of the DOS. In both solutions, the major categories contributing to the total investment costs are remnant treatment, cultivation, and dewatering. 
Utility costs account for the largest share in operating costs. Furthermore, biodiesel sales and electricity generation bring the most revenue.

Table 7. Computational times (CPUs) for robust optimal solutions with various budgets.

\begin{tabular}{|c|c|c|c|c|c|c|c|c|c|c|c|c|c|c|}
\hline $\begin{array}{c}\text { Budget of } \\
\text { supply and } \\
\text { demand }\end{array}$ & \multicolumn{14}{|c|}{ Budget of price uncertainty } \\
\hline & 0 & 1 & 2 & 3 & 4 & 5 & 6 & 7 & 8 & 9 & 10 & 11 & 12 & 13 \\
\hline 0 & 3.8 & 3.3 & 3.1 & 3.3 & 3.4 & 3.3 & 3.3 & 3.4 & 3.2 & 3.2 & 3.2 & 3.2 & 3.5 & 3.3 \\
\hline 1 & 3.6 & 3.4 & 3.2 & 3.2 & 3.3 & 3.3 & 3.5 & 3.5 & 3.2 & 3.2 & 3.3 & 3.3 & 3.4 & 3.2 \\
\hline 2 & 1.4 & 1.3 & 1.2 & 1.2 & 1.2 & 1.2 & 1.2 & 1.1 & 1.3 & 1.2 & 1.3 & 1.3 & 1.3 & 1.2 \\
\hline
\end{tabular}

Table 8. Upper and lower bounds of each iteration to obtain the robust optimal solution IROS.

\begin{tabular}{cccccc}
\hline $\begin{array}{c}\text { Outer } \\
\text { iteration }\end{array}$ & $\begin{array}{c}\text { ROI } \\
(\%)\end{array}$ & $\begin{array}{c}\text { Inner } \\
\text { iteration }\end{array}$ & $\begin{array}{c}\text { Upper } \\
\text { bound }\end{array}$ & $\begin{array}{c}\text { Lower } \\
\text { bound }\end{array}$ & Gap \\
\hline \multirow{2}{*}{1} & 0 & 1 & 16.31 & $-1.21 \times 10^{3}$ & $1.22 \times 10^{3}$ \\
& 0 & 2 & 4.81 & 4.81 & 0 \\
2 & 0.85 & 1 & 11.50 & $-1.21 \times 10^{3}$ & $1.22 \times 10^{3}$ \\
& 0.85 & 2 & 0 & 0 & 0 \\
\hline
\end{tabular}

In Table 7, all optimal solutions in Figure 24 can be returned in 5 seconds. The upper and lower bounds for solving the IROS is shown in Table 8. The proposed solution algorithm takes two outer iterations to solve $F(r)=0$. For each outer iteration, the column-and-constraint generation algorithm requires two inner iterations to find the worst-case uncertainty realization. When the proposed algorithm solves $F(r)$, the best upper bound based on the optimal solution of the master problem keeps decreasing as more uncertainty realizations are identified by 
the subproblem. On the other hand, the best lower bound increases when the subproblem is given a new first-stage decision in each inner loop. The gap between the best upper and lower bounds in the inner loop reduces rapidly until the stopping criterion is satisfied. As two-stage ARMIFP problems cannot be solved by any off-the-shelf solver, the short computation times and small numbers of iterations for the entire algorithm to converge indicate that the proposed solution strategy represents an efficient algorithm for the proposed two-stage ARMIFP problem.

\subsubsection{Maximizing productivity}

Another important fractional metric to measure the performance of processing pathways is productivity, which is defined as the ratio of the mass flow rate of the biofuel product divided by the mass flow rate of the biomass from the dewatering section [236]. If the objective function of (AROM) is to maximize productivity, both the numerator and denominator of productivity involve only second-stage decision variables. As a result, the objective function of the auxiliary parametric function in the proposed algorithm contains only second-stage decision variables. Furthermore, this model involves only network configuration constraints and mass balance constraints, thus subject to only the supply and demand uncertainty. We present the subproblem and master problem in Appendix D. We solve the problem 
with various budgets of supply and demand uncertainty, and obtain only one optimal objective function value. The optimal processing pathway selects high pressure homogenization and sodium-methoxide-catalyzed transesterification and achieves the maximum productivity of $25.94 \%$. These results are the same with those obtained in the IROS. Since demand uncertainty does not cause infeasibility for the processing pathway with the highest productivity (otherwise the entire optimization problem is infeasible), the second-stage decisions are fully adjustable. Consequently, given linear mass balance constraints, the robust optimal solutions are able to scale linearly to obtain the same highest productivity as the biofuel demand fluctuates.

The obtained robust optimal ROIs show that current algae-based processing pathways are not competitive from an investment point of view. However, through the cost breakdown of two optimal solutions, we identify the bottleneck to be the high capital costs of cultivation, harvesting \& dewatering, and remnant treatment. Therefore, ongoing research into algae conversion technologies should concentrate on reducing the capital costs of these technologies. Additionally, the efficiency and cost-effectiveness will be sufficiently improved if stable microalgae species with higher oil productivity and higher biomass product concentration are found applicable to large-scale cultivation. With growing global consensus on 
utilizing sustainable fuels and products, algae-based processes may attract more potential investors in the future.

\subsection{Summary}

We developed by far the most comprehensive processing network with 46,704 alternative processing pathways for producing a plethora of algal fuels and valueadded bioproducts. We formulated a deterministic MIFP model to maximize the ROI of producing biofuels and bioproducts from microalgae. In order to handle parameter uncertainty in the deterministic model, we proposed a two-stage ARMIFP problem for the robust optimal processing pathway in the proposed processing network. Since the problem could not be solved by any off-the-shelf solver, we developed a tailored solution strategy that integrated a parametric algorithm with a column-and-constraint generation algorithm. Two optimal processing pathways were identified. The robust optimal processing pathway selected open pond, poly-electrolyte-based flocculation, filtration, high pressure homogenization, supercritical $\mathrm{CO}_{2}$ extraction, sodium-methoxide-catalyzed transesterification, PHB production, and direct combustion for biogas utilization. It was also found that the robust ROIs ranged from $0.35 \%$ to $7.83 \%$ corresponding to different budgets of uncertainty. Biodiesel price influenced the ROI most significantly. The highest productivity of $25.94 \%$ was found. The major 
contributors to the total investment cost were the capital costs of cultivation, harvesting, and remnant treatment. Future research should be directed to reducing the capital costs of these technologies and seeking microalgae species with higher oil productivity and higher biomass product concentration.

\subsection{Appendix A: Superstructure configuration description}

There are four technology alternatives provided in the cultivation section: open pond, flat plate photobioreactor (PBR), bubble column PBR, and tubular PBR. Open ponds are less costly to construct and operate, while they require more land area and are less productive than PBRs. Despite the differences in conversion coefficients and production costs, the immediate product from the first section contains too much water for downstream utilization. Therefore, in the subsequent sections, the algal biomass concentration must reach at least $30 \mathrm{wt} \%$ through a set

of dehydration operations. The harvesting section aims at reaching a concentration of $10 \mathrm{wt} \%$ via autoflocculation and dissolved air flotation, and we consider six alternative flocculants: poly-electrolyte, sodium hydroxide, poly-aluminum chloride, aluminum sulfate, chitosan, and poly- $\gamma$-glutamic acid. In the next section, both pressure filtration and centrifugation are able to increase the concentration of algae biomass product to $30 \mathrm{wt} \%$, and this slurry is suitable for wet-extraction methods in the next section. In addition, energy-intensive technologies, such as 
freeze drying and thermal drying, generate algae powder with a concentration of 85 wt $\%$. This product can then be processed by dry-extraction methods. To better utilize algal lipids, a cell disruption section destroys the biological protection layers and improves extraction efficiency. We consider five cell disruption alternatives. Bead beating is employed for both algae powder and algae slurry, while microwaving, high pressure homogenization, and sonication are considered only for algae slurry. Since the increased revenue from extra lipids may not compensate the increased capital expenditure (CAPEX) and operation expenses (OPEX) for cell disruption, we add one bypassing option in this section. Six technology alternatives are included in the lipid extraction section. Hexane extraction and supercritical $\mathrm{CO}_{2}$ extraction apply to both algae powder and algae slurry; isopropanol/hexane extraction is considered only for algae powder; butanol extraction is specifically for algae slurry. In addition to the above extraction-based technologies, we include an independent processing pathway for hydrothermal liquefaction (HTL), which can process dilute algal product from the harvesting section and achieve relatively higher lipid yields. Two groups of technologies are able to convert algal lipids to biofuels: transesterification results in biodiesel and glycerol, while hydroprocessing results in renewable diesel as a substitute to fossil-based diesel fuels. Specifically, we consider alkaline in-situ transesterification, acidic in-situ transesterification, 
and enzymatic in-situ transesterification for algae powder, sodium-methoxidecatalyzed transesterification, supercritical methanol transesterification, heterogeneous acid-catalyzed transesterification, enzymatic transesterification, Co/Mo-catalyzed hydroprocessing, and Ni/Mo-catalyzed hydroprocessing for lipids from various extraction technologies, and HZSM-5-catalyzed hydroprocessing for lipids from HTL. If a transesterification technology is selected in the biofuel production section, the coproduced glycerol can be utilized later to produce hydrogen, propylene glycol, glycerol-tert-butyl ether, and PHB. Moreover, we incorporate three hydrogen production alternatives, namely steam reforming, autothermal reforming, and aqueous-phase reforming. Their detailed processes were described in detail elsewhere [22]. In the last section, the algal remnant is sent to anaerobic digesters and converted to biogas, a recyclable aqueous product, and a separable solid product. Biogas is utilized onsite to produce methanol or electricity, and the off-gas is reused in the cultivation section. Multiple fuel and chemical products can be generated in the superstructure, which allows selecting a portfolio of products for the most economically favorable performance. 


\subsection{Appendix B: Economic evaluation constraints and definition of} ROI

$$
\begin{aligned}
L C & =l p \cdot \sum_{j \in J} \frac{Q O_{l, j, 15}}{\text { pro }_{j}} \\
S C & =s c c \cdot \sum_{i \in I, j \in J} C C_{i, j} \\
W C & =w c c \cdot\left(\sum_{i \in I, j \in J} C C_{i, j}+L C+S C\right)
\end{aligned}
$$

Land cost $L C$ is calculated by Equation (1.B1), where $I p$ denotes the unit land cost and proj $_{j}$ is the productivity of technology $j$. The startup cost $S C$ and the working capital $W C$ are calculated by Equations (1.B2) and (1.B3), respectively. The capital cost, land cost, startup cost, and working capital constitute the total investment cost.

$$
\begin{aligned}
F C= & h \cdot \sum_{i \in I, j \in J, k \in K} f p_{k} \cdot M_{i, j, k}^{\text {makeup }} \\
U C= & h \cdot e p \cdot \sum_{i \in I, j \in J, k \in K}\left(u p c o_{i, j, k} M_{i, j, k}^{\text {out }}+u p c i_{i, j, k} M_{i, j, k}^{i n}\right) \\
& +h \cdot h p \cdot \sum_{i \in I, j \in J, k \in K}\left(u h c o_{i, j, k} M_{i, j, k}^{\text {out }}+u h c i_{i, j, k} M_{i, j, k}^{i n}\right) \\
& +h \cdot c p \cdot \sum_{i \in l, j \in J, k \in K}\left(u c c o_{i, j, k} M_{i, j, k}^{\text {out }}+u c c i_{i, j, k} M_{i, j, k}^{i n}\right) \\
& +h \cdot w p \cdot \sum_{i \in I, j \in J, k \in K E} M_{i, j, k}^{\text {emisions }}
\end{aligned}
$$

The feedstock cost $F C$ is evaluated by Equation (1.B4), where $h$ represents the annual operating hours and $f p_{k}$ is the feedstock cost of species $k$. Later, we calculate 
the utility cost $U C$ in Equation (1.B5), where $e p, h p, c p$, and $w p$ denote the prices of electricity, heating utility, cooling utility, and waste treatment, respectively. upco $i_{i, j, k}$, uhco $o_{i, j, k}$, and $u c c o_{i, j, k}$ are the unit consumption rates of electricity, heating utility, cooling utility, respectively, for species $k$ in the "out" flow of technology $j$ in section i. upci $i_{j, j, k}$, uhci $i_{j, j, k}$, and $u c c i_{j, j, k}$ have similar definitions but based on the "in" flow.

$$
\begin{aligned}
& O C=c p i c \cdot(w \cdot n o \cdot h \cdot c o+t a+c l) \\
& M C=m c c \cdot \sum_{i \in I, j \in J} C C_{i, j} \\
& O O C=o o c c l+o o c c 2 \cdot \sum_{i \in I, j \in J} C C_{i, j} \\
& P T I=p t i c \cdot \sum_{i \in I, j \in J} C C_{i, j} \\
& D C=d c c \cdot \sum_{i \in I, j \in J} C C_{i, j}
\end{aligned}
$$

Other production expenditures are calculated by Equations (1.B6)-(1.B10). Operations cost $O C$ consists of three parts: operators' wages-based cost, technical assistance cost ta, and cost of laboratory $c l$. In Equation (1.B6), w, no, and co are the wage per operator, the number of operators, and the coefficient for related costs, respectively. Inflation is captured by the CPI (consumer price index) coefficient cpic. The maintenance cost $M C$, property tax and insurance PTI, and depreciation cost $D C$ are evaluated based on the total capital cost and respective 
coefficients $m m c$, ptic, and $d c c$. Operating overhead cost $O O C$ consists of a part oocc1 based on the operations cost, and a part oocc 2 based on the maintenance cost.

$$
\begin{aligned}
& S=h \cdot\left(\sum_{i \in I, j \in J, k \in K} p p_{k} \cdot M_{i, j, k}^{\text {product }}+e p \cdot u p g \cdot M_{8,2,17}^{\text {in }}\right) \\
& G E=g e c \cdot S
\end{aligned}
$$

The sales $S$ of selling the products are calculated by Equation (1.B11), where $p p_{k}$ denotes the product price of species $k$ and upg is the unit electricity generation rate. A general expense $G E$ is spent for promotion and sales management, and it is evaluated as a portion, gec, of the total sales in Equation (1.B12).

$$
R O I=\frac{(1-t r) \cdot(S-F C-U C-O C-M C-O O C-P T I-D C-G E)}{\sum_{i \in I, j \in J} C C_{i, j}+L C+S C+W C}
$$

The goal of this deterministic problem is to obtain the maximum ROI, which is a widely used indicator to project financing. As shown in Equation (1.B13), ROI is defined as the net earnings divided by the total capital investment [46]. The net earnings are further defined as the after-tax difference between the annual sales and OPEX. The latter quantity is the sum of feedstock costs, utility cost, operations cost, maintenance cost, operating overhead cost, property taxes, insurance, and depreciation cost. 


\subsection{Appendix C: Monotonicity of $F(r)$}

The goal of this section is to show that the third level maximization problem of $F(r)$ is a monotonic function with respect to several uncertain parameters considered in the second level minimization problem. The monotonicity of the maximization problem can be used to simplify the original uncertainty set by eliminating the absolute value functions.

By definition, $M, c 1 \geq 0$, and $c 2, c 31, c 32, c 33, c 42, c 43, c 5 \leq 0$.

Let $G\left(T 1_{k}, T 2_{k}, T 31, T 32, T 33, T 5\right)=\max _{M \in O} N_{2}\left(T 1_{k}, T 2_{k}, T 31, T 32, T 33, T 5, M\right)$, where $N_{2}$ is defined in (AROM) and the uncertain parameters are determined by Constraints (1.29)-(1.43).

Lemma 1. $\forall k \in K: G\left(T 1_{k}, T 2_{k}, T 31, T 32, T 33, T 5\right)$ is monotonically increasing with respect to $T 1_{k} ; G\left(T 1_{k}, T 2_{k}, T 31, T 32, T 33, T 5\right)$ is monotonically decreasing with respect to $T 2_{k}, T 32, T 33$, and $T 5$, respectively.

Proof. (1) $\forall k \in K: \exists T l_{k}^{l} \geq T l_{k}^{2},\left(T_{l}, T l_{k}^{l}\right)$ and $\left(T_{l}, T l_{k}^{2}\right)$ are two feasible points in uncertainty set $U$ with the values of remaining components denoted by $T_{1}$ being the same. Let $M$ be the optimal solution of $G\left(T_{1}, T l_{k}^{2}\right)$. We have 


$$
\begin{aligned}
& G\left(T_{1}, T l_{k}^{1}\right)-G\left(T_{1}, T l_{k}^{2}\right) \\
& =\max N_{2}\left(T_{1}, T l_{k}^{1}, M\right)-\max N_{2}\left(T_{1}, T l_{k}^{2}, M\right) \\
& \geq N_{2}\left(T_{1}, T l_{k}^{1}, M^{*}\right)-N_{2}\left(T_{1}, T l_{k}^{2}, M^{*}\right) \\
& =c l \cdot M_{k}^{\text {product, }}\left(T l_{k}^{1}-T l_{k}^{2}\right) \geq 0 .
\end{aligned}
$$

Therefore, $G\left(T 1_{k}, T 2_{k}, T 31, T 32, T 33, T 5\right)$ is monotonically increasing with respect to $T 1_{k}$

(2) $\forall k \in K: \exists T 2_{k}^{l} \geq T 2_{k}^{2},\left(T_{2}, T 2_{k}^{l}\right)$ and $\left(T_{2}, T 2_{k}^{2}\right)$ are two feasible points in uncertainty set $U$ with the values of remaining components denoted by $T_{2}$ being the same. Let $M^{*}$ be the optimal solution of $G\left(T_{2}, T 2_{k}^{2}\right)$. We have

$$
\begin{aligned}
& G\left(T_{2}, T 2_{k}^{l}\right)-G\left(T_{2}, T 2_{k}^{2}\right) \\
& =\max N_{2}\left(T_{2}, T 2_{k}^{1}, M\right)-\max N_{2}\left(T_{2}, T 2_{k}^{2}, M\right) \\
& \geq N_{2}\left(T_{2}, T 2_{k}^{1}, M^{* *}\right)-N_{2}\left(T_{2}, T 2_{k}^{2}, M^{* *}\right) \\
& =c 2 \cdot M_{k}^{\text {makeup }, * *}\left(T 2_{k}^{1}-T 2_{k}^{2}\right) \leq 0 .
\end{aligned}
$$

Therefore, $G\left(T 1_{k}, T 2_{k}, T 31, T 32, T 33, T 5\right)$ is monotonically decreasing with respect to $T 2 k$. Similarly, we can prove that $G\left(T 1_{k}, T 2_{k}, T 31, T 32, T 33, T 5\right)$ is monotonically decreasing with respect to $T 32, T 33$, and $T 5$, respectively.

\subsection{Appendix D: Subproblem and master problem formulation of maximizing productivity}

(SUB2) Min Objective function defined in (1.57)

s.t. Constraints (3.71)-(3.74) 


$$
\begin{aligned}
-P 7_{i, j, k} & +P 14_{i, j, k} \geq 0, \quad \forall i \in I, j \in J, k \in K \\
P 2_{4, j, 15} & +P 6_{4, j, 15}-\sum_{k^{\prime}} P 6_{4, j, k^{\prime}} \cdot p_{4, j, k^{\prime}, 15}^{i n}+P 7_{4, j, 15}-P 8_{4, j, 15} \\
& -\sum_{k^{\prime}} P 8_{4, j, k^{\prime}} \cdot s c_{4, j, k^{\prime}, 15} \cdot x_{4, j} \geq-r, \quad \forall j \in J \\
P 2_{i, j, k}+ & P 6_{i, j, k}-\sum_{k^{\prime}} P 6_{i, j, k^{\prime}} \cdot p_{i, j, k^{\prime}, k}^{i n}+P 7_{i, j, k}-P 8_{i, j, k}-\sum_{k^{\prime}} P 8_{i, j, k^{\prime}} \cdot s c_{i, j, k^{\prime}, k} \cdot x_{i, j} \\
\geq & 0, \quad \forall j \in J,(i, k) \notin(4,15) \\
P 1_{i, j, k}+ & P 8_{i, j, k}+P 9_{i, j, k}-\sum_{k^{\prime}} P 10_{i, j, k^{\prime}} \cdot s f_{i, j, k^{\prime}, k}^{\text {down }}-P 11_{i, j, k} \cdot s f_{i, j, k}^{e m i s s i o n l} \\
& -P 12_{i, j, k} \cdot s f_{i, j, k}^{p r o d u c t} \geq 0, \forall i \in I, j \in J, k \in K
\end{aligned}
$$

Constraints (3.78)-(3.80)

$-P 9_{i, j, k}+P 11_{i, j, k} \geq 0, \quad \forall i \in I, j \in J, k \in K$

$-P 9_{i, j, k}+P 12_{i, j, k}+P 15_{k} \geq 1, \quad \forall i \in I, j \in J, k \in K P$

$-P 9_{i, j, k}+P 12_{i, j, k} \geq 0, \quad \forall i \in I, j \in J, k \notin K P$

Constraints (3.87)-(3.100)

$\omega_{k}, T 6, T 7_{k}, T 8_{k} \in\{0,1\}, P 1_{i, j, k}, P 2_{i, j, k}, P 13, P 14_{i, j, k} \geq 0, \forall i, j, k$

(MA2) $\max \eta$

s.t. Constraints (3.1)-(3.7)

$\eta \leq \sum_{i \in I, j \in J, k \in K P} M_{i, j, k, s}^{\text {product }}-r \cdot \sum_{j \in J} M_{4, j, 15, s}^{i n}, \quad \forall s \in S$

Constraints (3.105)-(3.119)

(1.D16) 


\section{CHAPTER 4 \\ CONSEQUENTIAL LIFE CYCLE OPTIMIZATION}

\subsection{Introduction}

In this study, we propose a general conceptual framework of consequential life cycle optimization (LCO). We demonstrate how the four phases of consequential life cycle assessment (LCA) are integrated into the optimization framework. To achieve this goal, we develop a general system boundary to enclose a target process and the processes and markets that would be influenced by the target process. Based on the general system boundary, we develop a multiobjective optimization model that integrates consequential LCA, techno-economic analysis, process models, and market models. The market models describe how a market reaches a new equilibrium by shifting its aggregate supply and/or demand curves. The resulting optimization model is formulated as a mixed-integer nonlinear programming (MINLP) model, which can be computationally intractable for general-purpose global optimization solvers. To tackle the computational challenge, a tailored global optimization algorithm is developed to integrate the inexact parametric algorithm and the branch-and-refine algorithm. We apply the proposed framework to a process design problem for algal renewable diesel production. A superstructure with a number of process and technology alternatives 
in each section of the algal process is developed. To establish the system boundary, we analyze the potential feedstock and product markets, and identify corresponding consequences. The resulting optimization model minimizes the 100year global warming potential (GWP) per GJ of algal renewable diesel and the ReCiPe endpoint score per GJ of algal renewable diesel as the environmental objective functions, and maximizes the net present value (NPV) per GJ of algal renewable diesel as the economic objective function. The constraints of the consequential LCO model systematically account for network configuration, mass and energy balances, market models, techno-economic evaluation, and environmental impact assessment. We compare the optimal environmental and economic results based on the proposed framework with those based on the existing attributional LCO framework. The major novelties of this work are summarized below:

- A novel general conceptual framework of consequential LCO, including a general system boundary and a general multiobjective optimization model;

- A tailored global optimization algorithm for efficiently solving the resulting large-scale nonconvex MINLP problem;

- A comprehensive application of the proposed framework to algal renewable diesel production with detailed market analysis;

The rest of the paper is organized as follows. The General Conceptual Framework section describe the four phases of consequential LCA and how they are integrated 
into an optimization model. Next, the proposed framework is applied to a process design problem for algal renewable diesel production. Conclusion is provided in the last section.

\subsection{General conceptual framework}

The goal of this work is to develop a general conceptual framework of consequential LCO. As consequential LCA has never been employed in an LCO model, we first show how the four phases of consequential LCA contribute to the consequential LCO framework, before we exhibit and describe the general form of the optimization model.

\subsubsection{Goal and scope definition}

The first phase is goal and scope definition, where we declare the key settings of the LCA, including but not limited to the goal of the study, functional unit, system boundary, allocation method, and major assumptions. Typical goals of consequential LCA include determining the consequential environmental impacts of a product over its life cycle, and identifying the hotspots of the consequential environmental impacts. The function of the application can be either producing a specified product, or performing a certain service. The functional unit is thus defined with both the qualitative and quantitative aspects of that function. By applying the same functional unit, results derived from different approaches can be 
fairly compared. Since consequential LCA captures marginal changes in upstream and downstream markets, multi-product allocation is automatically handled by system expansion.

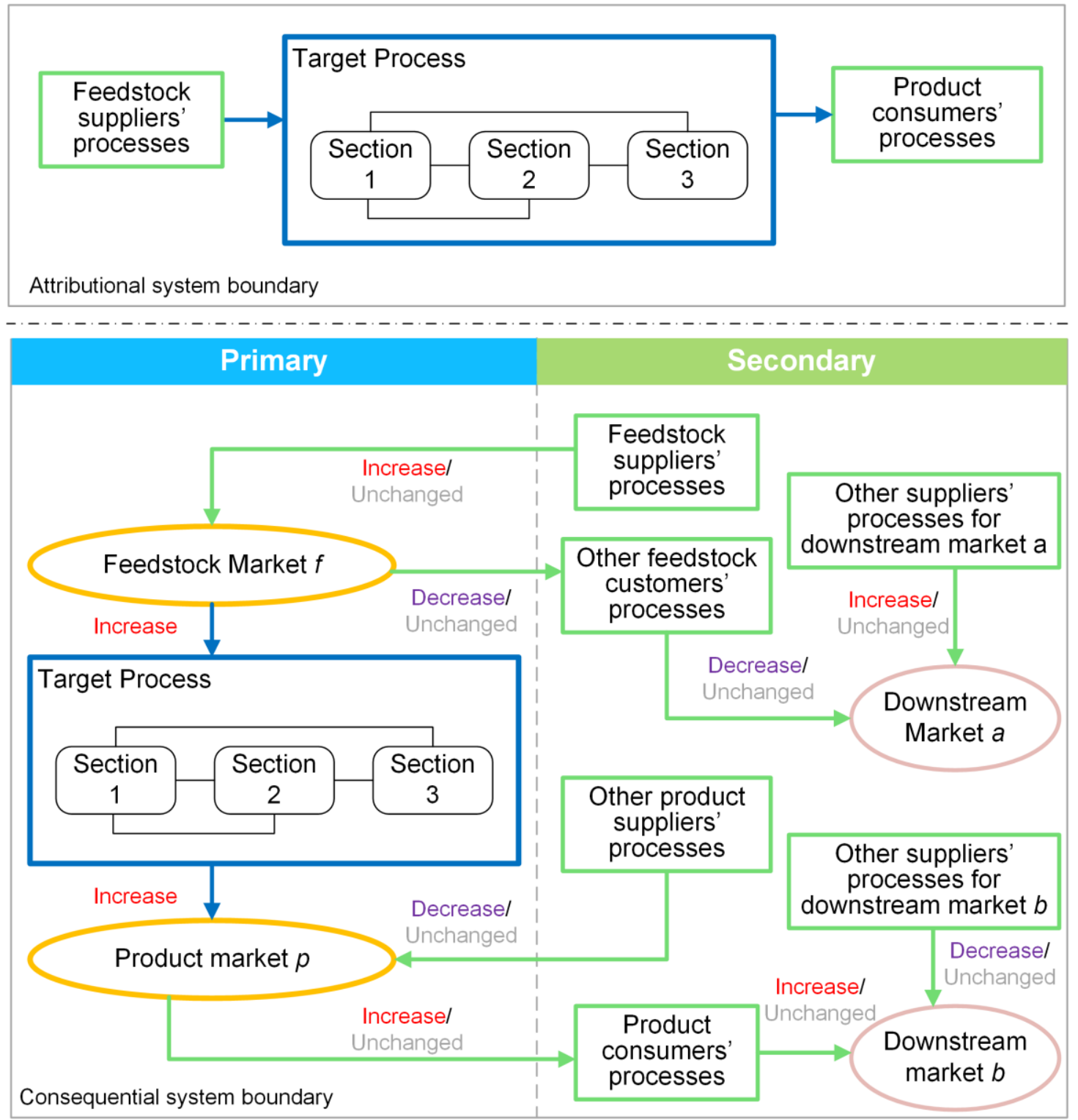

Figure 23. System boundary of attributional LCA (upper) and consequential LCA (lower). 
A major difference between attributional LCA and consequential LCA lies in the system boundary. As shown in the upper part of Figure 23, the system boundary in a conventional attributional LCA study includes only the directly connected processes. A general system boundary of consequential LCA is shown in the lower part of Figure 23, which consists of two regions and three types of components. The primary region contains the target process, feedstock and product markets, and linking flows. The secondary region contains affected processes, downstream markets, and linking flows. It is noted that there can be multiple feedstock and product markets in the primary region and multiple downstream markets in the secondary region. For illustrative purposes, only one representative market is presented for each category.

Once the target process starts operations, its demand for feedstocks and its supply for products begin to influence the relevant markets. According to classical microeconomics [237], when the demand or supply of a good changes, the price and quantity produced of that good in its market shift to a new market equilibrium. Since a good in the primary region is also an input or output of other processes in the secondary region, the change in price and quantity of that good will also affect the product price and quantity in the downstream markets, and so on. Given different price elasticities of supply and demand, there can be different 
consequences regarding the change in the target process. Possible consequences are denoted as "Increase", "Decrease", and "Unchanged" in Figure 23.

The assumptions concerning the components in the general system boundary include:

(1) In line with all market equilibrium-based models [68], the system is assumed at an equilibrium state before the change occurs and can converge to a new equilibrium state after the change occurs.

(2) In the secondary region, the supplies from the feedstock suppliers' processes, other product suppliers' process, and other suppliers' processes for downstream markets are perfectly elastic. The assumption is in accordance with the assumption for consequential life cycle inventory (LCI) and life cycle impact assessment (LCIA) results in the Ecoinvent database [238].

(3) As consequences can be theoretically infinite, it is practical to simplify the consequences to be analyzed [69]. Normally, no more than two levels of markets and no more than one affected technology are identified and discussed [72, 95]. It is assumed in this chapter that the consequences propagate no further than the suppliers of the downstream markets. Moreover, the downstream markets are assumed to be unaffected by the changes that are not included in this chapter. As a result, the demands of downstream markets in the secondary region are perfectly inelastic. 


\subsubsection{Life cycle inventory analysis}

During the second phase, all the LCI data, e.g. material and energy flow rates, are collected. In both attributional and consequential LCA, the mass and energy balance relationships within a process can be readily established following existing mathematical models. In addition, consequential LCA requires the changes in the involved markets be quantified and used together with the process flow data. However, collecting these data is not a trivial task.

Unlike relatively mature attributional LCA approaches, namely process-based, input-output-based, and hybrid LCA [58, 239], consequential LCA is still under development. Consequential LCI data measure the difference between the physical flows after a change occurs and those before the change occurs in the system. Although researchers share a common understanding of the advantages of consequential LCA, there is no procedural consensus. As models and techniques are highly simplified representations of reality, they are unable to accurately capture all possible changes in the system [240]. Despite the limitation, existing consequential LCA methods and applications still provide valuable insights that cannot be obtained from the corresponding attributional LCA results. There are four methods to determine the types and amounts of materials to be included in the consequential LCI data, namely scenario analysis, simple PE models, 
sophisticated PE models, and CGE models. Applying different assumptions and targeting various levels of markets and geological regions, these methods coexist in the literature for varying modeling needs [241]. Since each method is capable of capturing only a subset of consequences (sometimes with overlaps), one method cannot prove to be superior over the others $[68,69]$.

In this chapter, we aim the consequential LCO framework at relatively small-scale applications, such as estimating the life cycle environmental impacts associated with producing fuels from a biorefinery. Therefore, the consequential LCI data for market changes are compiled using tailored simple PE models due to its accuracy and flexibility. The detailed reasons are summarized as follows.

- Systematic approach: A scenario analysis predetermines the affected quantities, such as prices and flow rates, in the market. Therefore, it considers only a limited number of consequences. In this chapter, a systematic approach is desired to determine the affected quantities along with other major system decisions. Therefore, the scenario analysis method is not chosen in the proposed framework.

- Model resolution: In order to capture a whole picture of a sector or even an entire economy, comprehensive models understandably reduce their complexity by considering similar products as one commodity. For example, different kinds of liquid fuels are lumped as one commodity in a CGE model [81]. Therefore, the results would be too coarse to make fair comparison with the results via the attributional approach which captures details between similar products. 
Additionally, it is impossible to differentiate the environmental impacts of the products in the same commodity category.

- Model maturity: There are only several sophisticated PE models available for non-agricultural sectors. Therefore, if changes involve products that are not relevant to agricultural sectors, existing sophisticated PE models are unable to determine the consequences. Moreover, a mapping is required to link the LCI data from solving PE or CGE models to the LCIA results in the Ecoinvent database [195]. However, a significant portion of the LCIA data are not available. In the case of biofuels, there are observed limitations, such as the lack of accurate land use modeling in Ecoinvent, the modelled processes not adapted to each region, and high uncertainty due to model aggregation [66].

In contrast, tailored simple PE models are immune to the drawbacks of the other methods and they are most suitable to be integrated in the proposed consequential LCO framework. The constraints of the proposed PE model are introduced in the consequential LCO model subsection.

\subsubsection{Life cycle impact assessment}

Next, consequential LCI data are translated into environmental impact indicators, or midpoint indicators, by multiplying the LCI data by the consequential characterization factors of corresponding impact categories. Based on the midpoint indicators, more comprehensive impact indicators, or endpoint indicators, can be 
calculated by summing up normalized midpoint indicators in different impact categories with a set of predefined weights. In the proposed consequential LCO model, either a midpoint or an endpoint indicator can be employed as the environmental objective function, as long as it can be calculated using the consequential LCI data and characterization factors.

\subsubsection{Interpretation}

Interpretation is the most versatile phase in consequential LCA. It is performed in every previous phase to guarantee its completeness and consistency. It works in an iterative manner before the conclusion is given. When interpreting the LCIA results, the major contributor in a certain category may be explained by tracking down the corresponding LCI data. Furthermore, the optimal environmental impacts and optimal economic indicators can be plotted as a Pareto-optimal curve. The curve contains many optimal solutions that demonstrate trade-offs between the objective functions and allow a visual comparison between the solutions from different approaches.

\subsubsection{Consequential LCO model}

Based on the settings in consequential LCA, a general consequential LCO model is proposed to determine simultaneously the optimal consequential environmental impact and economic performance. As shown below, the model consists of two 
objective functions and four sets of constraints. The environmental objective function and economic objective function are given by (1.17) and (1.18), respectively. The mass and energy balance of the target process is modeled by Constraint (1.19); the aggregate supply and demand are calculated by Constraint (1.20) and (1.21), respectively; the new market equilibrium is established by Constraints (1.22).

$$
\begin{array}{ll}
\min & \sum_{k}\left[c f d_{k} \cdot u_{k}\left(X_{k}\right)\right]+\sum_{l}\left(c f t_{l} \cdot Q_{l}\right)+\sum_{l, r, s}\left[c f c_{l, r, s} \cdot v_{l, r, s}\left(Q_{l}, A S_{l}, A D_{l}\right)\right] \\
\max & \sum_{k, l} h_{k, l}\left(P_{l}, Q_{l}, X_{k}\right) \\
\text { s.t. } \quad Q_{l}=\sum_{k} f_{k, l}\left(X_{k}, Y P_{k}\right), \quad \forall l \\
\quad A S_{l}=m_{l}\left(Q_{l}, P_{l}, Y S_{l}\right), \quad \forall l \\
A D_{l}=n_{l}\left(Q_{l}, P_{l}, Y D_{l}\right), \quad \forall l \\
A S_{l}=A D_{l}, \quad \forall l
\end{array}
$$

In this model, the involved species are indexed by $k$, the feedstock and product markets by $l$, the affected processes by $r$, and the downstream markets by $s$. The environmental objective function is to minimize the total environmental impact change in the system. $c f d_{k}$, $c f t$, and $c f_{C l, t, s}$ are the characterization factors for emitting material $k$, transporting feedstock or product $l$, and producing material $s$ in process $r$ in market $l$, respectively. Continuous variable $X_{k}$ can be used to model various flow rates of material $k$ in the target process. Function $u_{k}(\cdot)$ denotes the 
flow rate of material $k$ in the direct emissions flow. $Q_{l}$ is the flow rate of feedstock or product $l$ in the target process. $A S_{l}$ is the aggregate supply of feedstock or product $1 . A D_{l}$ is the aggregate demand of feedstock or product 1 . Function $V l, r_{s}(\cdot)$ denotes the flow rate change of material $s$ in process $r$ relevant to feedstock or product 1 . The economic objective function is to maximize NPV of the project. Function $h_{k, \lambda}(\cdot)$ denotes the cost estimation function. $P_{l}$ is the price of feedstock or product 1 . Constraint (1.19) represents a general process model denoted by function $f_{k, l}(\cdot)$. Integer variable $Y P_{k}$ can be introduced to model the selection of technology alternatives in the target processes. The tailored PE model to quantify the changes in the market consists of Constraints (1.20)-(1.22). More specifically, the model describes how the supply and demand relationships in the relevant markets are influenced given the flow rates of the feedstocks and products in the target processes. The feedstock and product flow rates of the target process are first integrated into tailored aggregate supply function $m(\cdot)$ in Constraint (1.20), and aggregate demand function $n(\cdot)$ in Constraint (1.21). Integer variable $Y S_{l}$ and $Y D_{l}$ can be introduced to facilitate evaluating aggregate supply and demand, respectively. In Constraint (1.22), a new equilibrium can be achieved in each market by letting the supply equal to the demand. 


\subsection{Application to algal renewable diesel production}

To illustrate the proposed consequential LCO framework, the optimal process design for algal renewable diesel production using the proposed framework is addressed in this section.

\subsubsection{Goal and scope definition}

There are two goals for this consequential LCA: (1) Assessing the cradle-to-grave consequential life cycle environmental impacts of renewable diesel production from microalgae Scenedesmus sp. [242]; (2) Comparing the life cycle environmental impacts via the attributional and consequential approaches. The functional unit is defined as $1 \mathrm{GJ}$ of renewable diesel produced from microalgae. In a resource assessment study [243], a large number of potential microalgae farm sites in the U.S. were ranked according to the biomass productivity and freshwater availability of each site. The sites in the Gulf Coast area in Texas showed the highest ranks. Following these results, the renewable diesel production process in this chapter is expected to be established in the gulf coast area in Texas. 


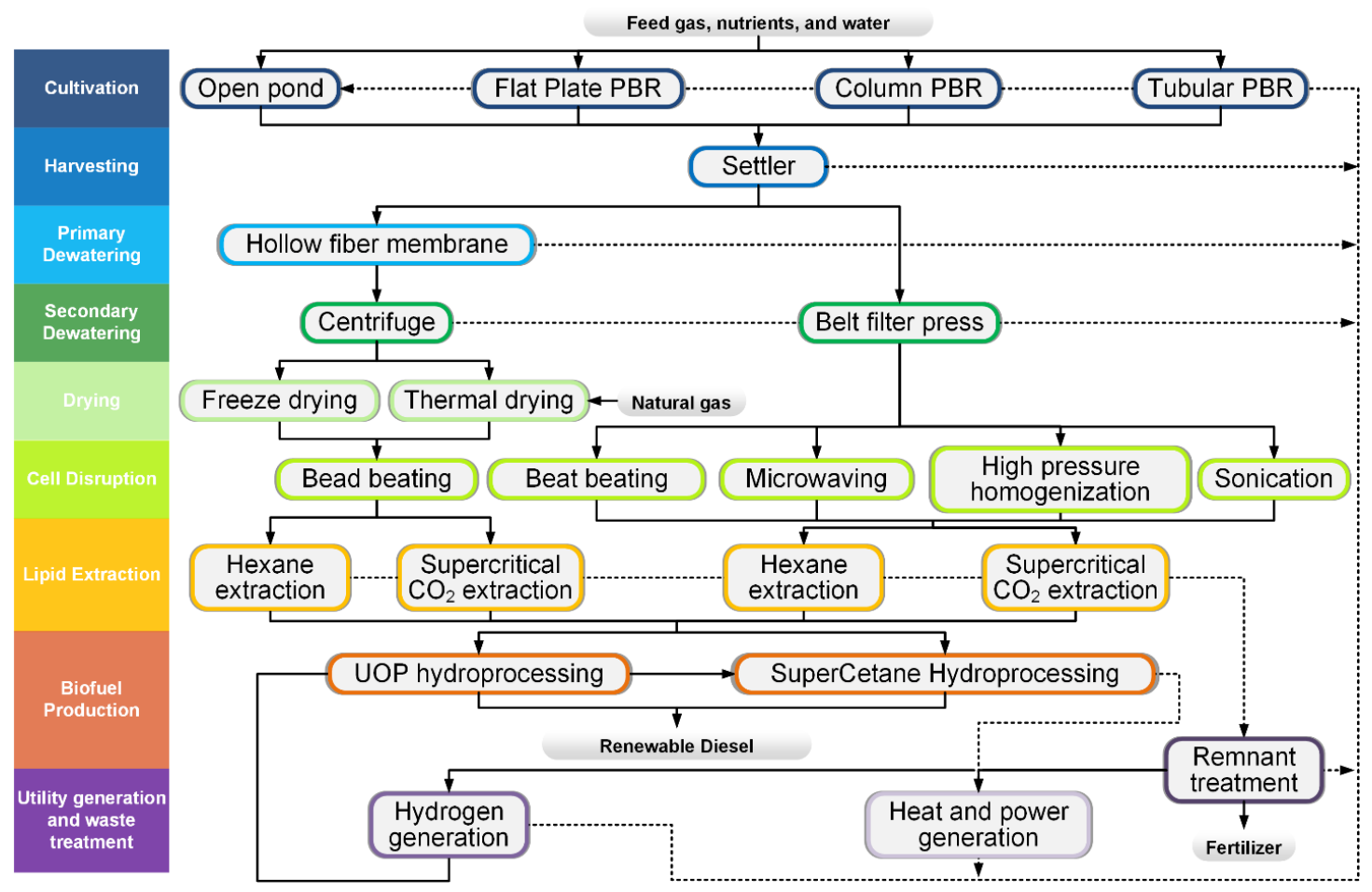

Figure 24. Superstructure of the algal renewable diesel production process.

The target process in this application converts a carbon gas source to algal renewable diesel. Many alternative process designs for renewable diesel production from microalgae are included in a superstructure as shown in Figure 24 and Figure 25. Figure 24 provides an overview of the technology alternatives in the superstructure, and Figure 25 shows the equipment units and material flows in the superstructure. There are 11 sections: cultivation, harvesting, primary dewatering, secondary dewatering, drying, cell disruption, lipid extraction, biofuel production, remnant treatment, utility generation, and hydrogen generation. As only one technology is included in the remnant treatment, utility generation, and hydrogen 
generation sections, they are aggregated as the final row in Figure 24 as utility generation and waste treatment. Renewable diesel is the major product of the algal renewable diesel production process and the byproducts include the digestate from the anaerobic digestion process in the remnant treatment section and the extra electricity in the utility generation section. In the remnant treatment section, an attractive alternative to the anaerobic digestion process is selling the algae meal directly as an animal feed. The use of algae meal as an animal feed can further reduce the amount of corn as the animal feed, thus mitigating the total environmental impacts from a consequential perspective. Despite its environmental advantage, selling the resulting algae meal does not generate additional carbon dioxide for the cultivation section as the anaerobic digestion process, thus reducing the ultimate yield of renewable diesel. In order to produce more renewable diesel, only anaerobic digestion is considered in the superstructure. 


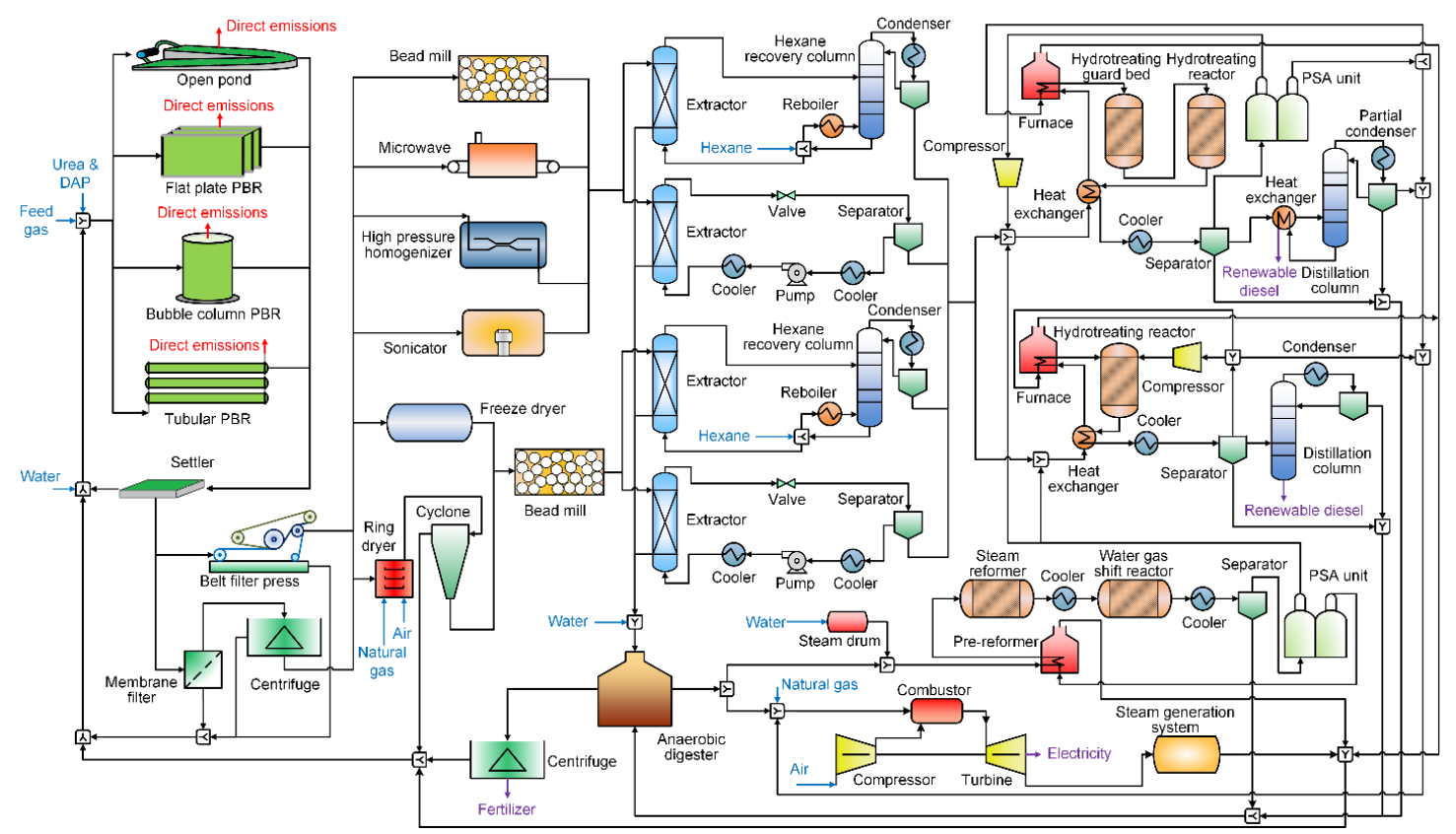

Figure 25. Detailed Superstructure of the algal renewable diesel production process.

The target process interacts with six markets to purchase feedstocks or sell products. Specifically, these markets trade urea, diammonium phosphate (DAP), natural gas, $n$-hexane, electricity, and diesel. Therefore, there are six markets included in the system boundary. In addition to the markets, the corresponding upstream processes and downstream processes as well as markets are also included in the system market as shown in Figure 23. Note that the total quantities of the goods in the considered markets are orders of magnitude larger than the quantities purchased/sold by the algal renewable diesel production process. However, the corresponding consequential LCA focuses on the changes to the upstream and downstream processes, which are relevant to the size of the system under study 
instead of the size of the markets. When the capacity of an algal renewable diesel production process is large enough to absorb the carbon gas source from a small power plant of $10 \mathrm{MW}$ [244], consequential LCA is preferred.

\subsubsection{Life cycle inventory analysis}

The consequences in each market as well as the tailored PE models for quantifying these consequences are presented in detail in this subsection.

- Urea market: Microalgae cultivation relies on two fertilizers: urea and DAP [36]. Urea is synthesized from ammonia and carbon dioxide. It is widely used as the nitrogen source for crops. New Orleans, Louisiana is a major gateway of imported urea in the U.S. and is where the urea market is located [245]. Two functions are required to define the equilibrium in the market. An aggregate supply function for the urea market can be developed using a cost curve for the urea delivered to the market [246]. However, there is no willingness-to-pay curve for the urea demand. Therefore, the definition of price elasticity of demand can be used to approximate the aggregate demand function [247]. Based on a recent urea price quote [248], the original equilibrium quantity of urea before the algal renewable diesel is produced can be calculated using the aggregate urea supply function. As the renewable diesel production process purchases additional urea from the market, the original equilibrium is shifted and the price and total quantity of urea in the market increase simultaneously. The changes in the urea market can propagate into both upstream and the 
downstream processes. The upstream processes increase their supplies and thus incur additional environmental impacts to the system. The consequences to the downstream processes are more complex than those to the upstream processes. Since the price of urea increases, the other customers purchase less urea than before. It has been shown that fertilization accounts for over $90 \%$ of the total use of urea [249], and corn consumes an order of magnitude more nitrogen fertilizers than other common crops [250]. Therefore, the reduction in the amount of urea can incur a decline in the corn production in the U.S. The relationship between corn yield and urea application rate is used to evaluate the amount of reduction in corn production $[251,252]$. In the downstream market for corn, the reduction in corn supplies is replenished by imported corn.

- DAP market: DAP, a phosphorus fertilizer, supplies both nitrogen and phosphorus to microalgae. An aggregate supply function for DAP can be formulated using an industry cost curve [253]. Therefore, the DAP market covers all the DAP consumed in the U.S. Next, the aggregate demand function for DAP can be constructed in the same manner as urea. Similar to the consequences of urea, the additional DAP consumed by the renewable diesel production process leads to simultaneous increases in the price and total quantity of urea in the market. The expanded upstream processes cause added environmental impacts. Approximately $90 \%$ of the phosphate products are consumed as fertilizers [254], and the majority of phosphorous fertilizers are used for corn, soybean, and wheat cultivation [250]. Accordingly, the decrease 
in the DAP demand by the other customers affects the production of corn, soybean, and wheat simultaneously. The declined amount of DAP is allocated to the production processes based on the DAP consumption rates of the crop production processes. Next, the decline in the crop production rates can be determined by the relationships between crop yields and their DAP application rates [252, 255-257]. Finally, the downstream markets for corn, soybean, and wheat are rebalanced by imported crops.

- Natural gas market: As introduced previously, there are a large number of alternative process designs included in the superstructure. The process design that demonstrates the best overall performance within the superstructure is termed the optimal process design. Natural gas will be consumed if an energy intensive technology such as freeze drying or thermal drying is selected in the optimal process design. An aggregate supply function and an aggregate demand function can be formulated according to the elasticity of supply and demand for natural gas, respectively [258]. Since the end use for pipeline quality shale gas is to generate heat, the change in the other customers' processes leads to a decrease in the heat supply, which is offset by increasing the heat generated by the heavy fuel oil from petroleum.

- $n$-Hexane market: $n$-Hexane serves as the solvent in two lipid extraction processes. As a portion of the solvent remains in the extraction products, $n$ hexane is continuously consumed and replenished in these processes. The size of the national market for $n$-hexane is relatively smaller than those of the other 
feedstocks, and additional demand can be satisfied domestically [259]. According to the U.S. Department of Health and Human Services [260], many facilities are capable of entering the market through a minor alteration in refining operating when the demand for $n$-hexane increases. Therefore, the increase in $n$-hexane demand can be completely absorbed by an expansion of the $n$-hexane suppliers' processes.

- Electricity market: The renewable diesel production process generates and consumes electricity simultaneously, and the rates of generation and consumption vary depending on the specific process design and operating conditions. As a result, the renewable diesel production process can influence the electricity market from both the customer's side and the supplier's side. The power grid in the state of Texas is managed by The Electric Reliability Council of Texas, or ERCOT, one of the nine independent systems operators in North America. An aggregate supply function can be developed using an ERCOT ISO supply stack curve [261]. As the price elasticity of demand for the electricity consumption in Texas is essentially zero [262], the downstream markets associated with the other electricity customers' processes are not influenced by the supply side. Therefore, the net import or net export of electricity in the renewable diesel production process would be completely absorbed by the electricity suppliers.

- Diesel market: Renewable diesel, the major product, is chemically identical to fossil-based diesel and compatible with existing engines and infrastructure. 
Although renewable diesel is comparatively environmentally sustainable, current commercial products are not economically competitive. In order to achieve regulatory goals, federal and state governments in the U.S. have offered incentives to renewable diesel suppliers, and its demand has been growing through increasing governmental and commercial fleet vehicle uses [263]. Therefore, renewable diesel products in the current stage are matched with specific demands and substitute for the fossil-based diesel products on an equivalent basis. In this chapter, the aggregate supply and aggregate demand functions in the diesel market can be modeled following the definition of price elasticity of supply and demand, respectively [264]. Given the aforementioned fact, introducing renewable diesel into the diesel market leads to a reduction in the demand for fossil-based diesel, thus resulting in a new market equilibrium. The change in the total diesel consumption (renewable diesel plus fossil-based diesel) can further influence the gasoline consumption in the transportation market [104], since both types of fuels are the major energy sources in the transportation sector.

\subsubsection{Life cycle impact assessment}

A well-recognized midpoint indicator for algal biofuels is GWP, which measures the global warming impact of a chemical relative to that of carbon dioxide, whose GWP is equal to 1 [265]. According to the Intergovernmental Panel on Climate Change, the latest 100-year GWPs of carbon dioxide and methane data are 1 and 
28, respectively [265]. Therefore, emitting $1 \mathrm{~kg}$ of methane is equivalent to emitting $28 \mathrm{~kg}$ of $\mathrm{CO}_{2}$ when evaluating the GWP of a system. Given its popularity in LCA for algal biofuels [266], the 100-year GWP is employed as the environmental objective in the first case study.

Since GWP focuses on a specific impact category, there is a need to determine the optimal process design that is environmentally benign from a variety of impact categories. Such a goal can be fulfilled by switching to an endpoint indicator as the environmental objective function. In the second case study, the ReCiPe endpoint score is used as the environmental objective function. This indicator incorporates a basket of impact categories and allows for a comprehensive evaluation of environmental impacts. The Hierarchist perspective and average weighting $(\mathrm{H}, \mathrm{A})$ are considered when collecting the endpoint scores of the ReCiPe method from the Ecoinvent database.

\subsubsection{Interpretation}

The optimal results of each case study are plotted as a Pareto-optimal curve, together with the breakdowns of the optimal environmental and economic objective function values of several representative points. The trade-offs between the representative points are analyzed. The results are later compared with those from the existing attributional LCO framework. The differences between the 
results are analyzed and pros and cons of consequential LCO are generalized. The details are provided in the later subsections.

\subsubsection{Problem statement and complete consequential LCO model}

In the optimization problem, we are given a comprehensive superstructure for producing renewable diesel from microalgae. We know the parameters for describing the mass and energy balance and analyzing the techno-economics of the process: the upper and lower bounds of the capacity of each technology; the composition of the feed gas; component concentrations in the inlet flows; the conversion of each reaction; stoichiometric coefficients of each reaction; the split fraction of each species to various flows; unit consumption and generation rates of power, heating, and cooling utilities for each technology; the total area limit and the area productivity of each bioreactor; coefficients for evaluating total project investment based on capital costs; coefficients for evaluating operating costs. We know that the markets for urea, DAP, natural gas, $n$-hexane, electricity, and diesel are influenced by the target process. Additionally, we are given the parameters to describe how the mass flow rate of each player changes in the market: original price and quantity of each good in the market; own price elasticity of demand and supply for a good, or a set of aggregate supply quantities of a good with regard to its market prices. We also know the input-output mass balance relationships of other 
processes linked to the markets. Moreover, we know the consequential characterization factors of every material purchased or released to the atmosphere, and type of energy consumed.

The goal is to determine the optimal process design for producing algal renewable diesel by simultaneously optimizing the economic and consequential environmental performance. The economic objective function is to maximize the NPV per GJ of algal renewable diesel, while the environmental objective function is to minimize the 100-year GWP per GJ of algal renewable diesel in the first case study and the ReCiPe endpoint score per GJ of algal renewable diesel in the second case study. The major decision variables include:

- Technology selection;

- The mass flow rate of each technology;

- The price of the good in each primary market;

- The aggregated supply and demand quantities of the good in each primary market.

The process model and techno-economic analysis model are based on previous studies [21, 267], and adapted to the current superstructure. The general model formulation of the algal renewable diesel application is given below. The complete consequential LCO model is provided in Appendix A.

$$
\max \quad O B J_{\text {economic }}=\frac{\sum_{i} a_{i}\left(X_{i}\right)^{n_{i}}+\sum_{m, n}\left(b_{m, n} \cdot P_{m} \cdot Q_{n}\right)+\sum_{j}\left(c_{j} \cdot Z_{j}\right)}{l s \cdot h \cdot T E C} \text { in Eq. (1.A64) }
$$


$\min \quad O B J_{\text {environmenal }}=\frac{T E I C}{T E}$ in Eq. (1.A80)

s.t. $\quad$ superstructure network configuration constraints (1.A81)-(1.A84)

mass balance constraints (1.A1)-(1.A21)

energy balance constraints (1.A22)-(1.A28)

market constraints (1.A29)-(1.A45)

techno-economic evaluation constraints (1.23)-(1.A64)

environmental impact assessment constraints (1.A65)-(1.A80)

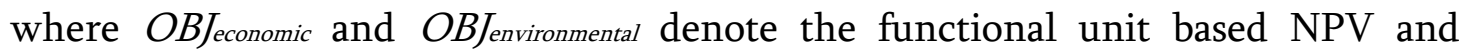

functional unit based environmental impact, respectively. The numerator of $O B J_{\text {economic }}$ is the NPV, which sums up a set of separable concave terms for the capital expenditures (CAPEX, denoted by $a_{i}\left(X_{i}\right)^{n_{i}}$ ), a set of bilinear terms for the revenue and a part of the operating expenditures (OPEX, denoted by $\left.b_{m, n} \cdot P_{m} \cdot Q_{n}\right)$, and several linear terms for the remaining OPEX (denoted by $c_{j} \cdot Z_{j}$ ). The numerator of $O B J_{\text {economic }}$ accounts for the total energy content of the renewable diesel product over the project lifetime. $l s, h$, and $T E C$ are the project lifetime, the operating time per year, and the energy content of the renewable diesel product per hour, respectively. The numerator of $O B \int_{\text {environmenal }}$ is the total environmental impact change TEIC for the GWP or the ReCiPe endpoint score. Since the market prices are dependent on the quantities of goods purchased/sold to the markets, the scale of the algal renewable diesel production process can affect the optimal economic and environmental performance. In order to account for the influence of the 
process scale, the flow rate of the feed carbon gas is modeled as a decision variable in the consequential LCO model.

The life cycle optimization model employs integer variables to select the technology/process alternatives and facilitate the process designs, and continuous variables for other decisions, such as process flow rates and market prices. A set of separable concave terms are introduced in the techno-economic evaluation constraints to calculate the CAPEX. Because the market prices and quantities sold/purchased are both continuous variables, a set of bilinear terms are used to calculate the revenue and the OPEX. Furthermore, the objective functions include mixed-integer fractional terms. Given the problem structure, the resulting problem is a mixed-integer nonlinear fractional programming (MINFP) problem and the global optimization of this problem is nontrivial.

\subsubsection{Tailored global optimization strategy}

Due to the combinatorial nature and nonconvexity of the MINFP problem, global optimization of the consequential LCO problem can be computationally intractable for general-purpose global optimization solvers [21]. To efficiently solve the resulting consequential LCO problem, we develop a tailored global optimization algorithm that integrates two state-of-the-art algorithms, including the inexact parametric algorithm [202] and the branch-and-refine algorithm [200, 268]. 


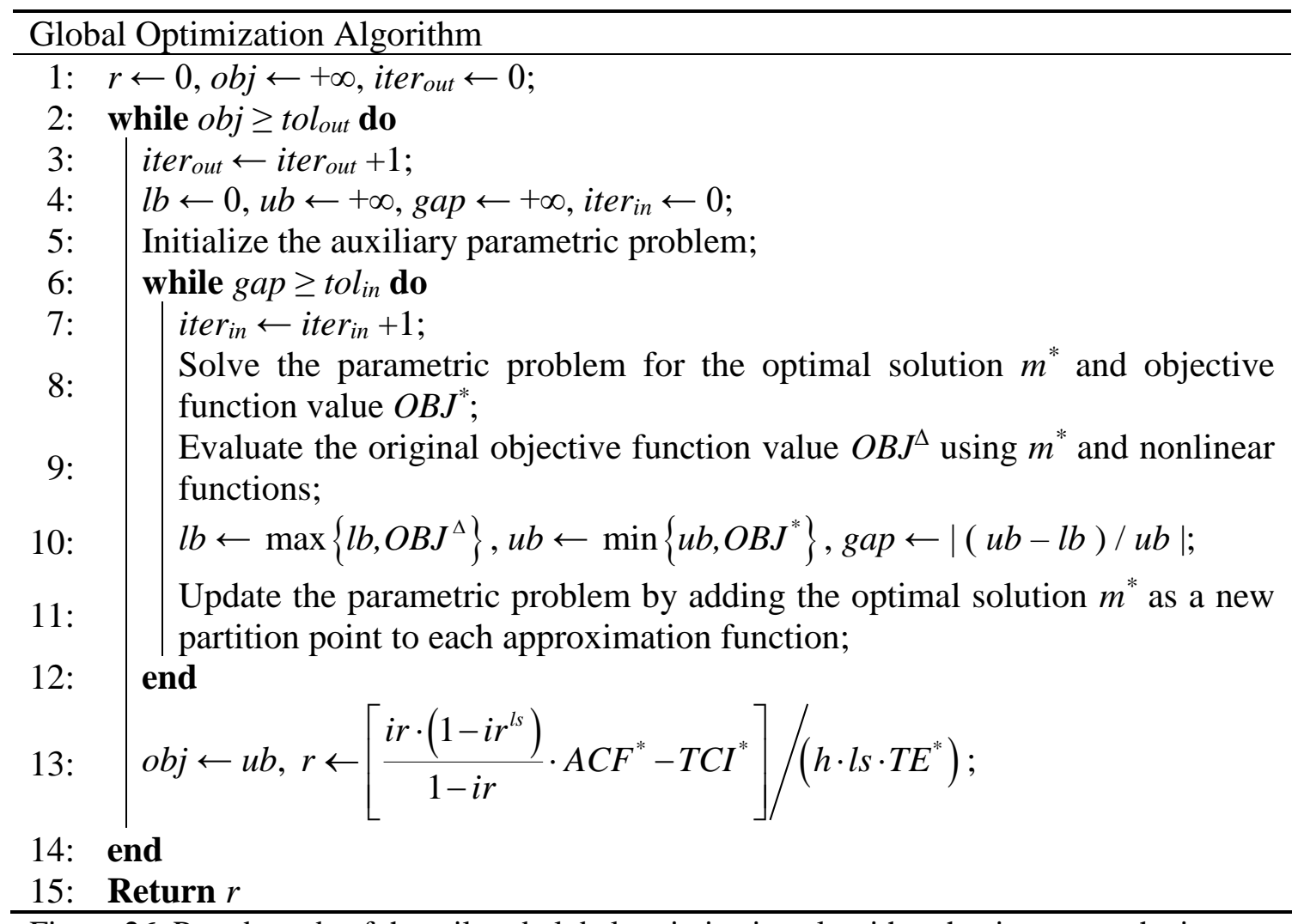

Figure 26. Pseudo code of the tailored global optimization algorithm that integrates the inexact parametric algorithm and the branch-and-refine algorithm.

The inexact parametric algorithm is used in the outer loop of the global optimization algorithm to handle the computational challenge stemming from the fractional objective functions. First, we introduce an auxiliary parametric problem $F(r)$ with respect to an auxiliary parameter $r$, so that the original optimal solution is identical to the optimal solution of the auxiliary parametric problem with the parameter $r^{*}$ such that $F\left(r^{*}\right)=0$ [202]. Even though no fractional term is included in the auxiliary parametric problem, $F(r)$ is still an MINLP problem with separable concave terms and bilinear terms in the objective function. To efficiently solve this 
MINLP problem, we replace the separable concave terms and bilinear terms with successive piecewise linear approximation functions, and solve the resulting mixed-integer linear programming (MILP) problems iteratively following the branch-and-refine algorithm $[200,268]$. This tailored solution algorithm is guaranteed to converge within finite iterations. Figure 26 presents the pseudo code of the tailored global optimization algorithm. iterout and iterin are the iteration counters of the outer loop and the inner loop, respectively. tolout and tolin are the optimality tolerance in the outer loop and the inner loop, respectively. The objective function value in the outer loop is denoted as $o b j$, while the upper bound and the lower bound in the inner loop are denoted as $u b$ and $l b$, respectively. gap represent the optimality gap in the inner loop. $m$ is the global optimal solution in the inner loop. The optimal objective function value and the evaluated objective function value are denoted as $O B J$ and $O B{ }^{\AA}$, respectively. The detailed model formulation of the auxiliary parametric problem is given in Appendix B.

\subsubsection{Results and discussion of case study 1}

GWP is selected as the life cycle environmental impact indicator in the first case study. The optimal solutions of the multiobjective optimization problem demonstrate the optimal economic performance and the optimal environmental performance simultaneously. The consequential LCO model accounts for the 
energy consumption and generation of each technology/process alternative in the superstructure. Note that heat integration can improve the energy efficiency of the resulting optimal process designs, but the detailed simulation models of the alternative processes in the superstructure are not available in the literature. It is worth future research to investigate the optimal performance of algae-based renewable diesel production considering heat integration.

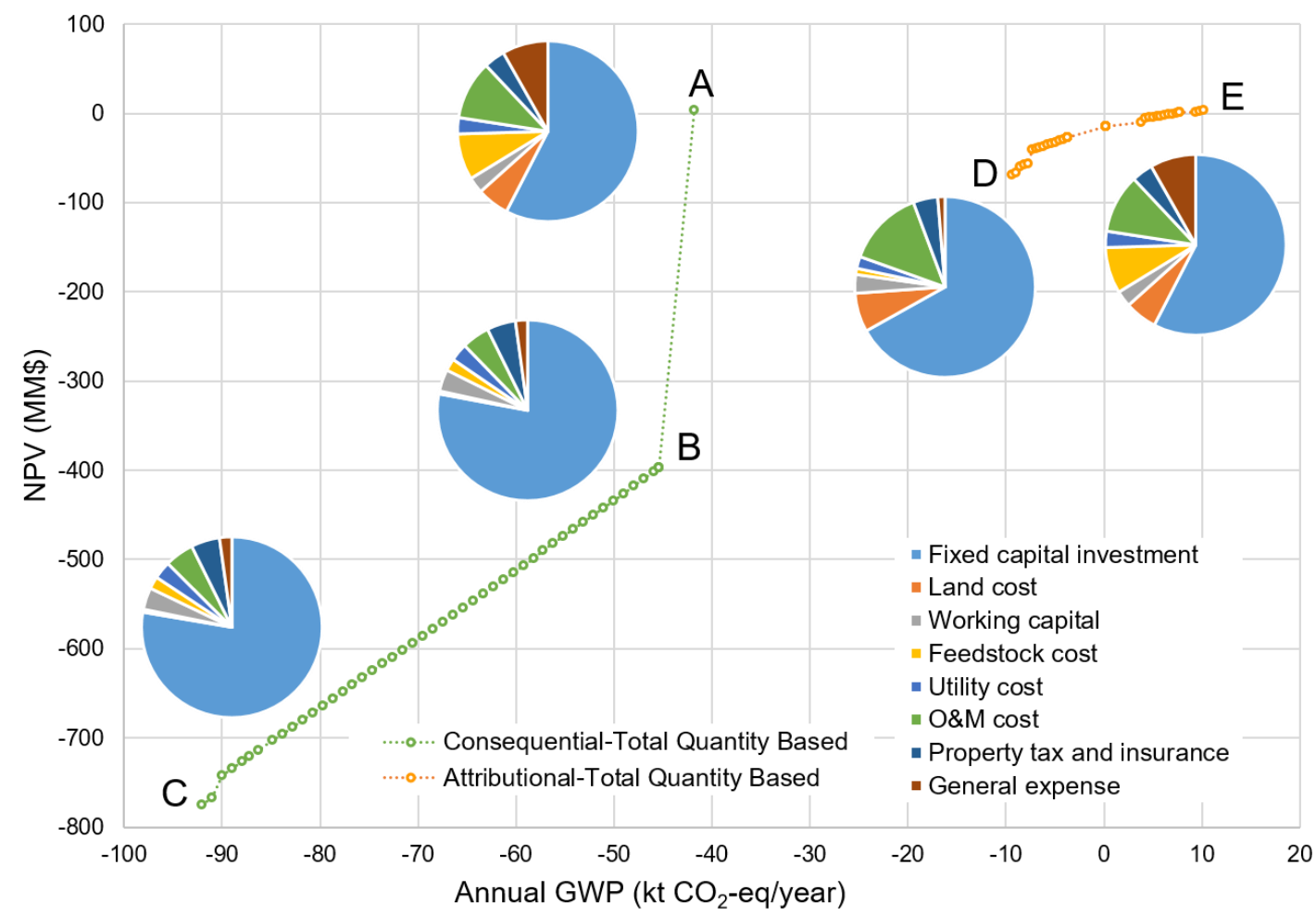

Figure 27. Pareto optimal profiles showing the trade-off between the NPV and the annual GWP. The five pie charts are the cost breakdowns of Solutions A-E. The functional unit based NPV values for Solutions A-E are \$0.4/GJ, \$-29.2/GJ, \$-28.5/GJ, \$-55.5/GJ, and \$0.4/GJ, respectively. The functional unit based GWP values for Solutions A-E are $-89,-67,-68$, -151 , and $22 \mathrm{~kg} \mathrm{CO}_{2}$-eq/GJ, respectively. The renewable diesel production capacities of Solutions A-E are 3.5, 5.0, 10.0, 0.5, and 3.5 million gallons per year, respectively. 
We first present the optimization results when the total economic and environmental performance are used as the objective functions. The corresponding optimal solutions are plotted as Pareto optimal profiles in Figure 27. A clear tradeoff can be identified between the optimal economic and environmental performance: the smaller the carbon footprint of an optimal design, the lower profit it earns. Among the optimal solutions, only Solution A has a positive NPV. The technologies selected by the most economically viable Solution A are shown in Figure 28.

The major differences between the technologies selected by Solution A and those with negative NPVs lie in the cultivation and dewatering sections. In the cultivation section, Solution A selects open ponds, while the other displayed optimal designs select flat plate photobioreactors (PBR). Open ponds show smaller production costs and smaller contributions to GWP than flat plate PBRs if they are employed to produce the same amount of algal biomass. However, the productivity of open ponds is significantly lower than that of flat plate PBRs. As the total area for the renewable diesel production process is limited, the largest amount of biomass generated by open ponds is substantially smaller than that by flat plate PBRs. 
The major feedstock of the renewable diesel production process is a carbon gas source from a power plant. Absorbing the carbon dioxide in the feedstock provides a significant credit for the direct emissions of the renewable diesel production process and the corresponding GWP is negative. If the resulting renewable diesel production process is environmentally sustainable due to the use of more energy efficient technologies and/or generating more fertilizer products, the total GWP can be negative. The negative GWP indicates that the renewable diesel production process is essentially a biosequestration system. Therefore, selecting flat plate PBRs enables production of the largest amount of renewable diesel, thus maximizing the net carbon dioxide reduction. In the dewatering sections, the most environmentally sustainable design of Solution C opts for a combination of a hollow fiber membrane in the primary dewatering section and a centrifuge in the secondary dewatering section, while Solution A selects a belt filter press for the whole dewatering process. Given the same target concentration in the dewatering product, a belt filter press is more cost effective than a combination of a hollow fiber membrane and a centrifuge, but the latter option consumes less energy and makes smaller contributions to GWP. 


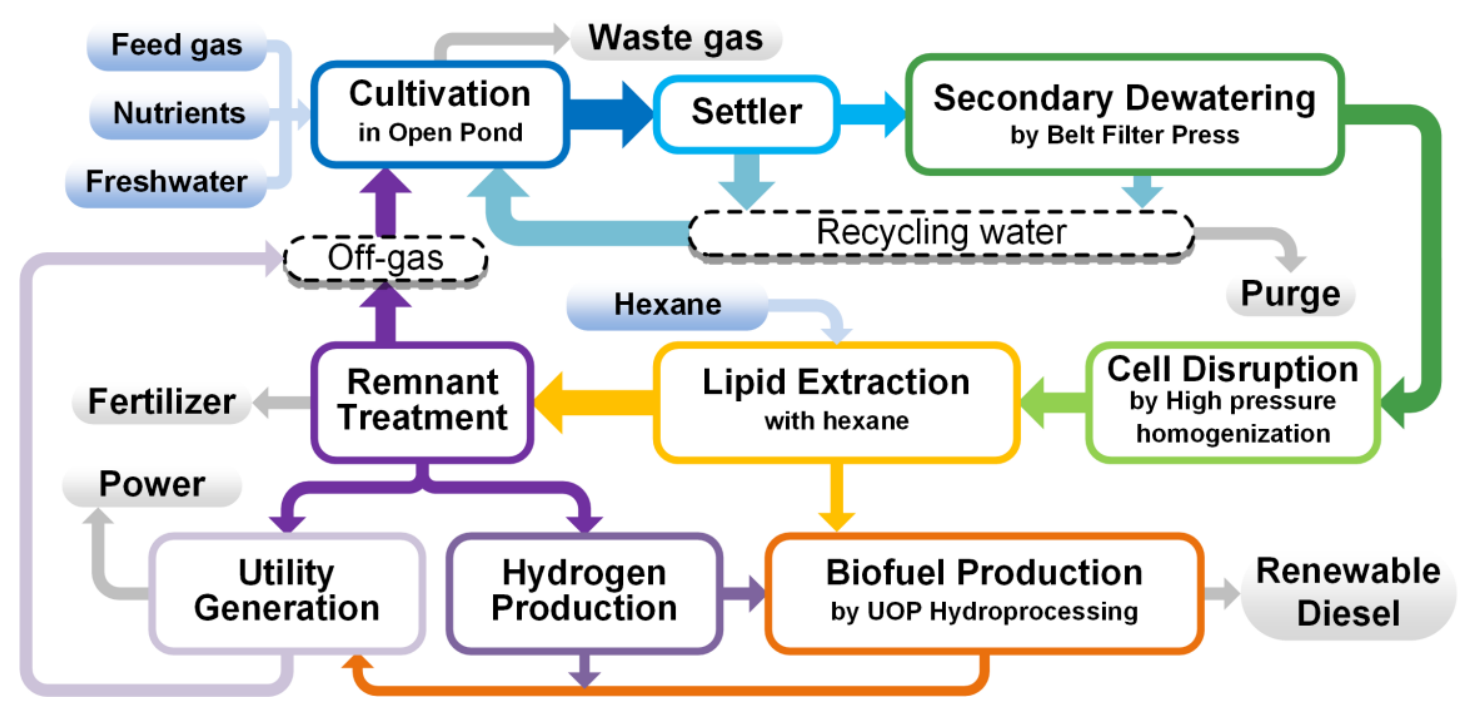

Figure 28. Optimal process pathway selected by Solution A and Solution E.

The cost breakdowns of three optimal solutions are also shown in Figure 27. Fixed capital investment is identified as the largest cost in all the Pareto-optimal designs, and its share increases as the annual GWP declines. Although cell disruption is energy intensive, about $77 \%$ of the electricity consumption can be satisfied by onsite power generation, and the net utility cost does not significantly impact the cost structure. 


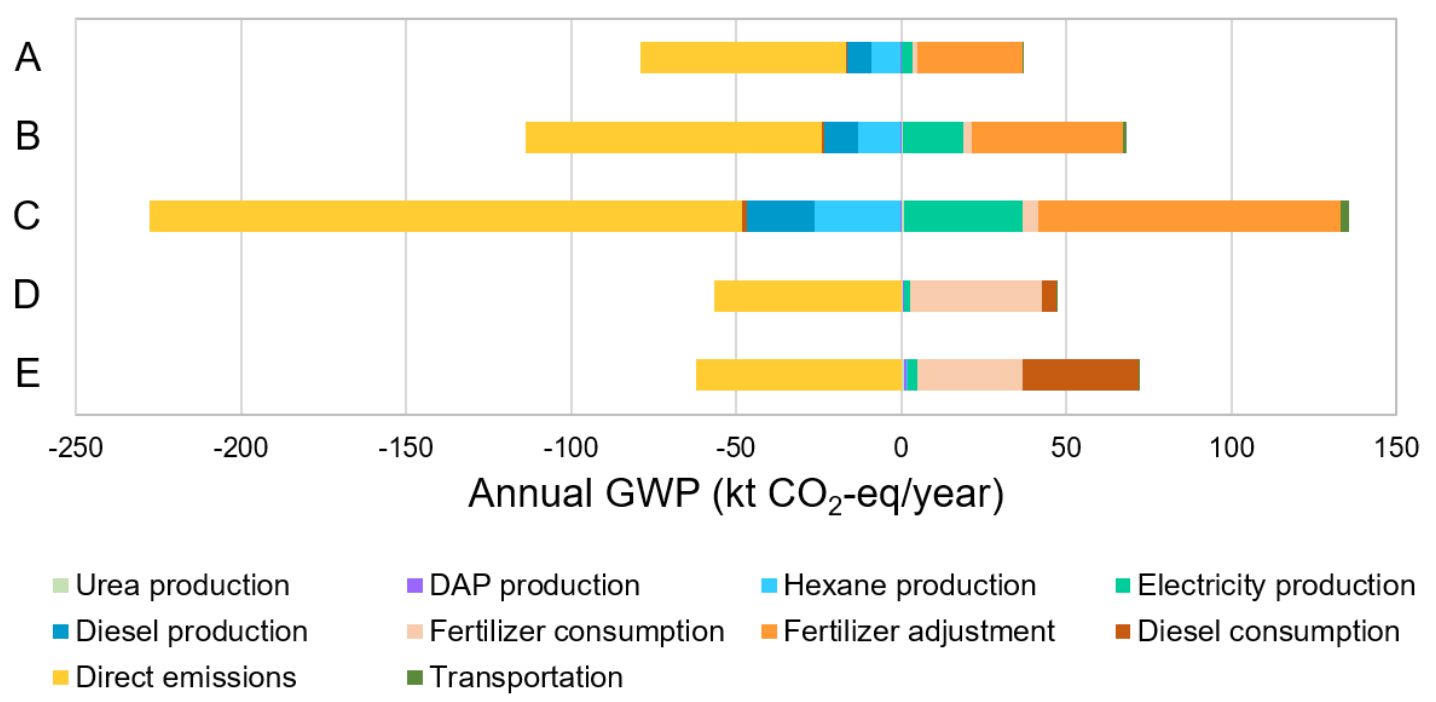

Figure 29. Annual GWP breakdowns of Solutions A-E in Figure 27. The functional unit based GWP values for Solutions A-E are $-89,-67,-68,-151$, and $22 \mathrm{~kg} \mathrm{CO}_{2}$-eq/GJ, respectively.

A major goal of this study is to compare the optimal environmental impacts by the proposed approach with those by the existing attributional approach. The Pareto optimal profile resulted from the existing attributional LCO approach is also presented in Figure 27. With the same optimal NPV, the annual GWP evaluated by the attributional approach is significantly larger than those by the consequential LCO approach. In order to better understand such a difference, the distributions of the annual GWP of Solutions A-E are presented in Figure 29. Solution A and Solution E select the same process layout and production level. Therefore, direct emissions, electricity production, and fertilizer-related contribution are the same in the corresponding designs. Note that "fertilizer consumption" in the attributional LCO approach and "fertilizer adjustment" in the consequential 
approach account for the end-of-life emissions of using the digestate as a nitrogen fertilizer. However, "fertilizer consumption" in the consequential LCO approach accounts for the environmental impacts associated with the changes in the downstream market due to a reduction in fertilizer consumption of the other customers.

The diesel-related contributions of the two optimal solutions are considerably different. In Solution E, the renewable diesel product is combusted in its end-oflife stage, thus contributing positively (denoted by "Diesel consumption") to the GWP of its entire life cycle from an attributional perspective. In contrast, a substitution effect is considered in Solution A, and the fossil-based market reaches a new equilibrium with a shifted aggregate demand curve. Since the reduction in demand causes the price to decline and the lower price encourages extra diesel consumption, the new total diesel consumption rate is in fact higher than the original total diesel consumption rate. Moreover, the additional diesel consumption offsets a portion of the gasoline consumption in the transportation sector. In the proposed model, the consequential LCI data account for (1) the consumption of renewable diesel, (2) the reduced production and consumption of displaced fossil-based diesel, (3) the increased production and consumption of fossil-based diesel due to a lower equilibrium price in the diesel market, and (4) the 
reduced production and consumption of gasoline in the transportation market. In the annual GWP breakdown of Solution A in Figure 29, "Diesel production" represents the net effect of (1) and (2). As a large amount of fossil-based diesel is displaced, the contribution is negative. In addition, "Diesel consumption" is the net result of (3) and (4). The small negative contribution from "Diesel consumption" shows that a substitution of diesel for gasoline can slightly reduce the GWP in the transportation sector.

Additionally, "hexane production" shows rather distinct contributions in Solutions $A$ and E. For Solution E, the consumption rate and the attributional characterization factor of hexane are both positive and relatively small. Therefore, the contribution of "hexane production" is positive but almost negligible in the breakdown of the attributional GWP results. In contrast, the consequential characterization factor of hexane from the Ecoinvent database is negative, and its absolute value is one order of magnitude larger than that of its attributional counterpart. The significant difference between the attributional and consequential characterization factors for producing hexane may stem from the use of more environmental sustainable technologies. With the same positive consumption rate of hexane, "hexane production" becomes negative in the consequential GWP results. 
The largest positive contribution in the consequential GWP results stems from "fertilizer adjustment", or the end-of-life emissions of utilizing the digestate. In order to further mitigate the environmental impacts from producing fuels from microalgae, future research should be directed to developing more sustainable technologies for handling digestate. If the conversion and utilization of digestate can be used for electricity production, the positive contribution to GWP would be negligible and the sustainable performance of this biofuel would be more pronounced.

The above consequential results suggest three key reasons why switching to algal renewable diesel mitigates the GWP: (1) sequestering the direct emissions in the feed gas, (2) reducing the consumption of fossil fuels, and (3) using hexane that creates additional GWP credits during its production phase. All three reasons are significant to the practical environmental benefits of renewable diesel from microalgae. However, the attributional approach considers the first one, but misinterprets the second with additional theoretical emissions and the third with static and theoretical characteristic factors. Overall, the consequential approach facilitates decision-making processes with more information taken into account. 


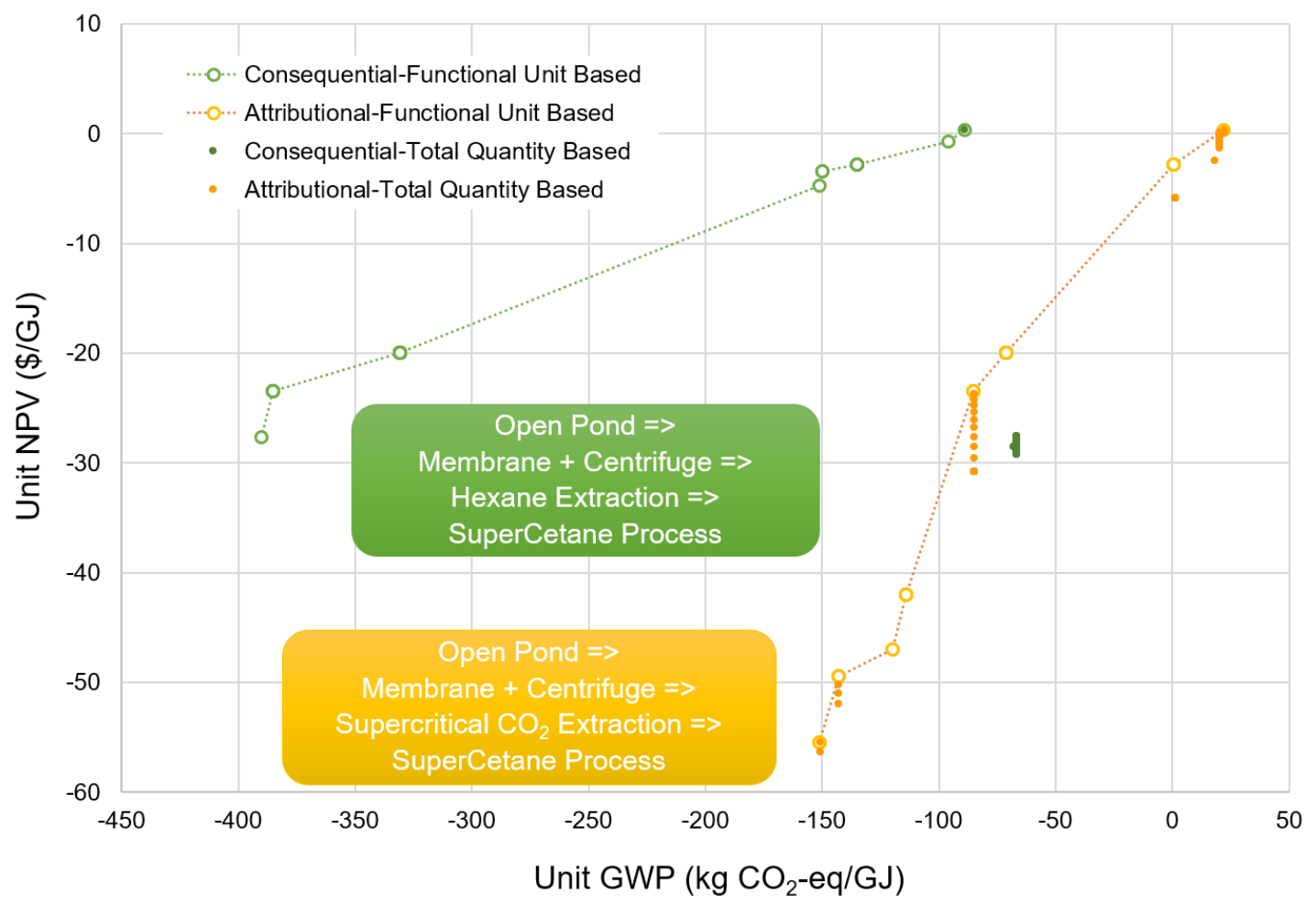

Figure 30. Pareto optimal profiles showing the trade-off between the functional unit based NPV and the functional unit based GWP. The boxes show the major process technologies for the optimal solutions with the minimum GWP per GJ of algal renewable diesel.

As the functional unit provides a fair basis for comparing results from different analysis approaches, functional unit-based values, rather than total quantity-based values, are often used and compared in the literature [197]. The Pareto optimal curves corresponding to functional unit based economic and environmental objective functions are presented in Figure 30. Before understanding the differences among the optimal solutions, it is important to compare the optimal results in the work with the corresponding results in the literature. With cultivation productivities ranging from 12 to $30 \mathrm{~g} / \mathrm{m}^{2} / \mathrm{d}\left(25 \mathrm{~g} / \mathrm{m}^{2} / \mathrm{d}\right.$ in this chapter), 
attributional well-to-wheel greenhouse gas emissions were reported to vary from 29 to $37 \mathrm{~kg} \mathrm{CO}$-eq/GJ of algal renewable diesel $[243,269,270]$. As only one process design was considered and evaluated in each of these attributional LCA studies, the environmental performance was nonoptimal. In this chapter, a set of process alternatives are compared automatically for the optimal environmental performance of producing algal renewable diesel, and all the optimal unit GWP results are smaller than $22 \mathrm{~kg} \mathrm{CO}$-eq/GJ.

The functional unit based results of the optimal solutions in Figure 27 are also demonstrated in Figure 30. The optimal solutions in Figure 27 are all located in the feasible regions, i.e., the regions under the Pareto optimal curves, in Figure 30. The most economically competitive solutions in Figure 30 are the same as those in Figure 27. However, the most environmentally sustainable solutions select different designs when functional unit-based objective functions are employed. Solution C selects flat plate PBRs in the cultivation section so that the largest amount of algal biomass can be produced in each year and the largest amount of off-gas can be sequestered. However, the corresponding process design consumes substantially more energy than those with open ponds, resulting in a relatively lower carbon mitigation efficiency. If the same amount of biomass is produced, relatively more off-gas would be sequestered by open ponds than by PBRs. 
Similarly, technologies selected by Solution $\mathrm{C}$ focus on increasing the capacity, while those in the most environmentally sustainable design focus on improving the efficiency. Overall, the results show that the environmental impacts based on the existing attributional approach are usually overestimated.

\subsubsection{Results and discussion of case study 2}

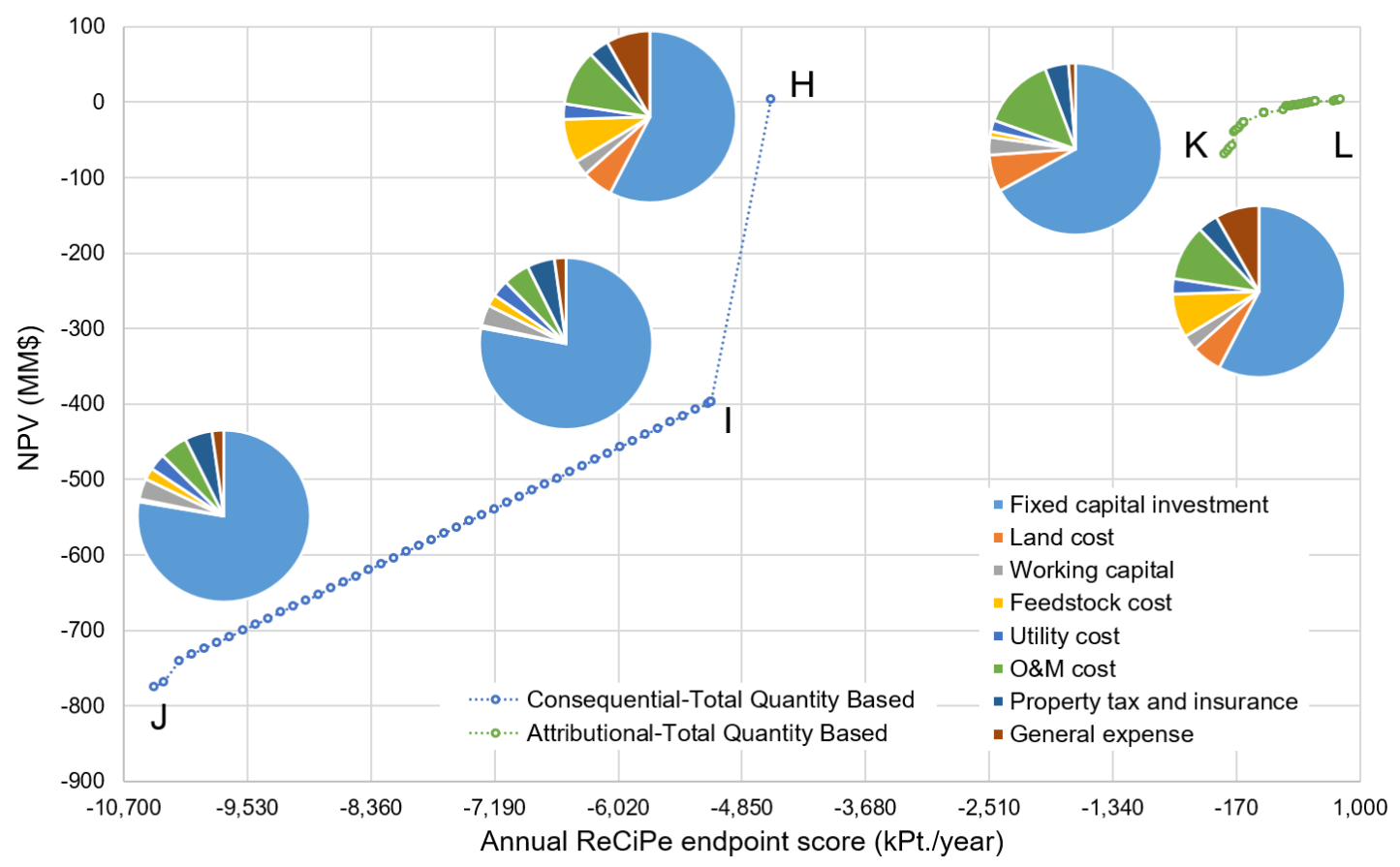

Figure 31. Pareto optimal profiles showing the trade-off between the NPV and the annual $\mathrm{ReCiPe}$ endpoint score. The five pie charts show the cost breakdown of Solutions H-L. The functional unit based NPV values for Solutions H-L are \$0.4/GJ, \$-29.2/GJ, \$-28.5/GJ, $\$-55.5 / \mathrm{GJ}$, and $\$ 0.4 / \mathrm{GJ}$, respectively. The functional unit based ReCiPe endpoint scores for Solutions $\mathrm{H}-\mathrm{L}$ are $-9.7,-7.6,-7.6,-4.5$, and $1.7 \mathrm{Pt} . / \mathrm{GJ}$, respectively. The renewable diesel production capacities of Solutions $\mathrm{H}-\mathrm{L}$ are 3.5, 5.0, 10.0, 0.5, and 3.5 million gallons per year, respectively.

The ReCiPe endpoint score is selected as the life cycle environmental impact indicator in the second case study. The Pareto optimal profiles for the total 
economic and environmental performance are shown in Figure 31. The annual ReCiPe endpoint scores via the consequential approach are substantially lower than those via the attributional approach given the same economic performance level. The breakdowns of the ReCiPe endpoints scores of Solutions $\mathrm{H}-\mathrm{L}$ are presented in Figure 32. "Fertilizer consumption", which is positive in Figure 29, becomes negative in Figure 32. As one of the consequences, more crops will be imported to account for the decreased supply if less fertilizer is applied to the production of domestic crops. Since the "agricultural land occupation" impacts of the imported crops are notably lower than those of the original domestic crops, the net effect of the change is a large reduction in this impact category. Therefore, the "fertilizer consumption" turns negative to illustrate an overall positive effect of importing crops.

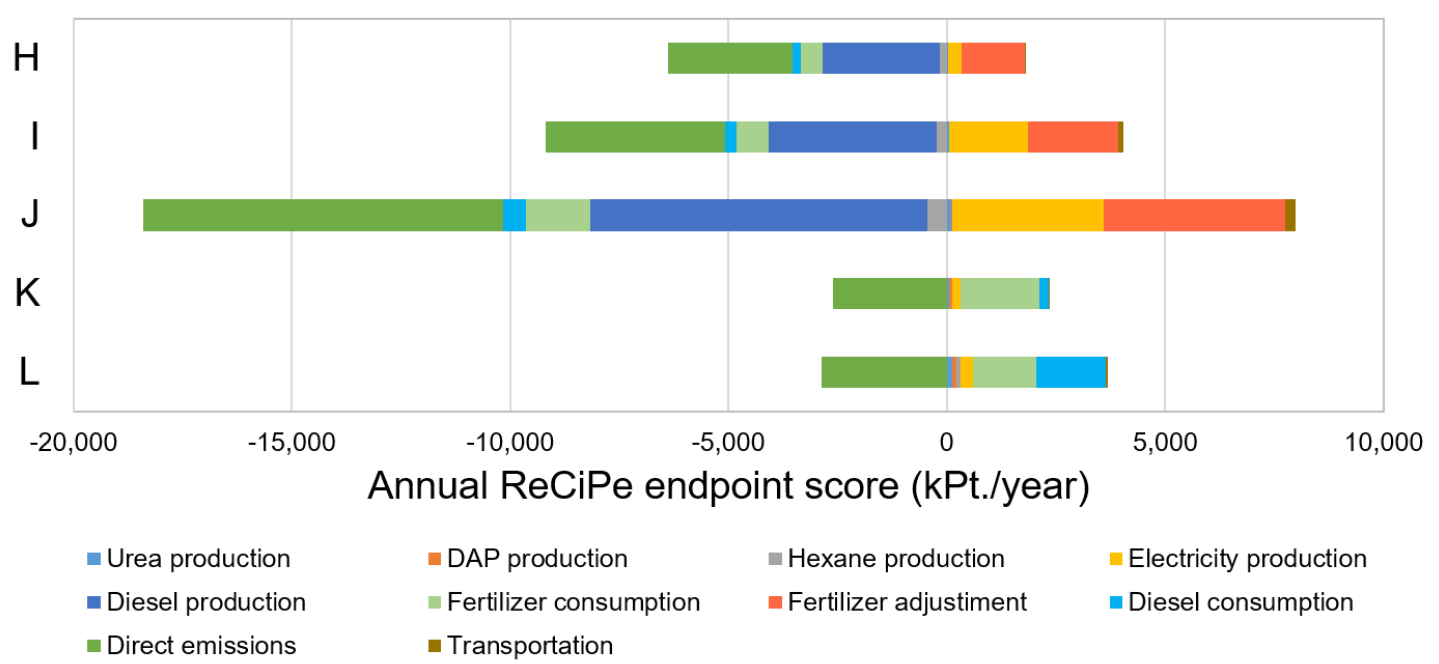


Figure 32. Annual ReCiPe endpoint score breakdowns of Solutions $\mathrm{H}-\mathrm{L}$ in Figure 31. The functional unit based ReCiPe endpoint scores for Solutions $\mathrm{H}-\mathrm{L}$ are $-9.7,-7.6,-7.6,-4.5$, and 1.7 Pt./GJ, respectively.

In order to identify the hotspots of the impact categories included in the ReCiPe endpoint scores, the total scores are decomposed by impact categories and components in Figure 33 for Solution $\mathrm{H}$ and Figure 34 for Solution L. The total height of each bar represents a $100 \%$ share and a contribution in each bar can be either positive or negative. In Figure 33, 10 out of 17 impact categories are most influenced by "diesel production", 2 impact categories by "fertilizer consumption", another 2 impact categories by "direct emissions", 1 impact category by "hexane production". In fact, such major components provide environmental credits in the corresponding impact categories. Diesel production that does not contribute to the attributional LCO approach proves to be a key component to mitigate the environmental impacts from a consequential perspective. A corresponding environmental profile for Design L is shown in Figure 34. The environmental hotspots are "power generation”, “DAP production”, and "end-of-life emissions". Moreover, these contributors are all positive and add to the environmental impacts. 


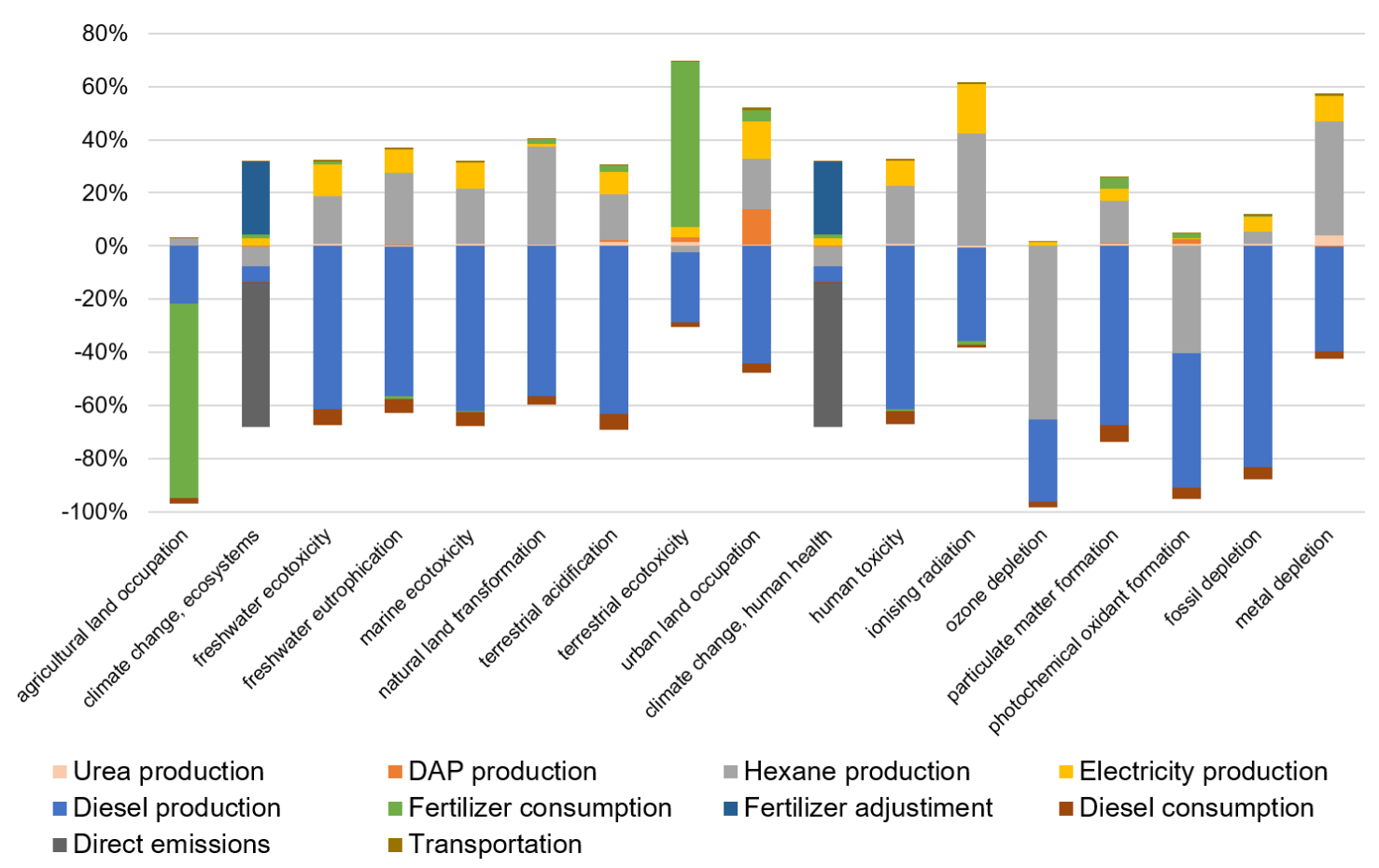

Figure 33. Environmental profile of Solution H in Figure 31.

Based on the hotspot analysis for attributional results, it is encouraged to mitigate the environmental impacts of the aforementioned components. Since many effects are completely overlooked in attributional LCA, there are notable discrepancies in the identified hotspots based on the consequential and attributional results. Therefore, if only the attributional approach is used for identifying the environmental hotspots, the results may overestimate the practical performance in several categories and underestimate the contributions from the others. 


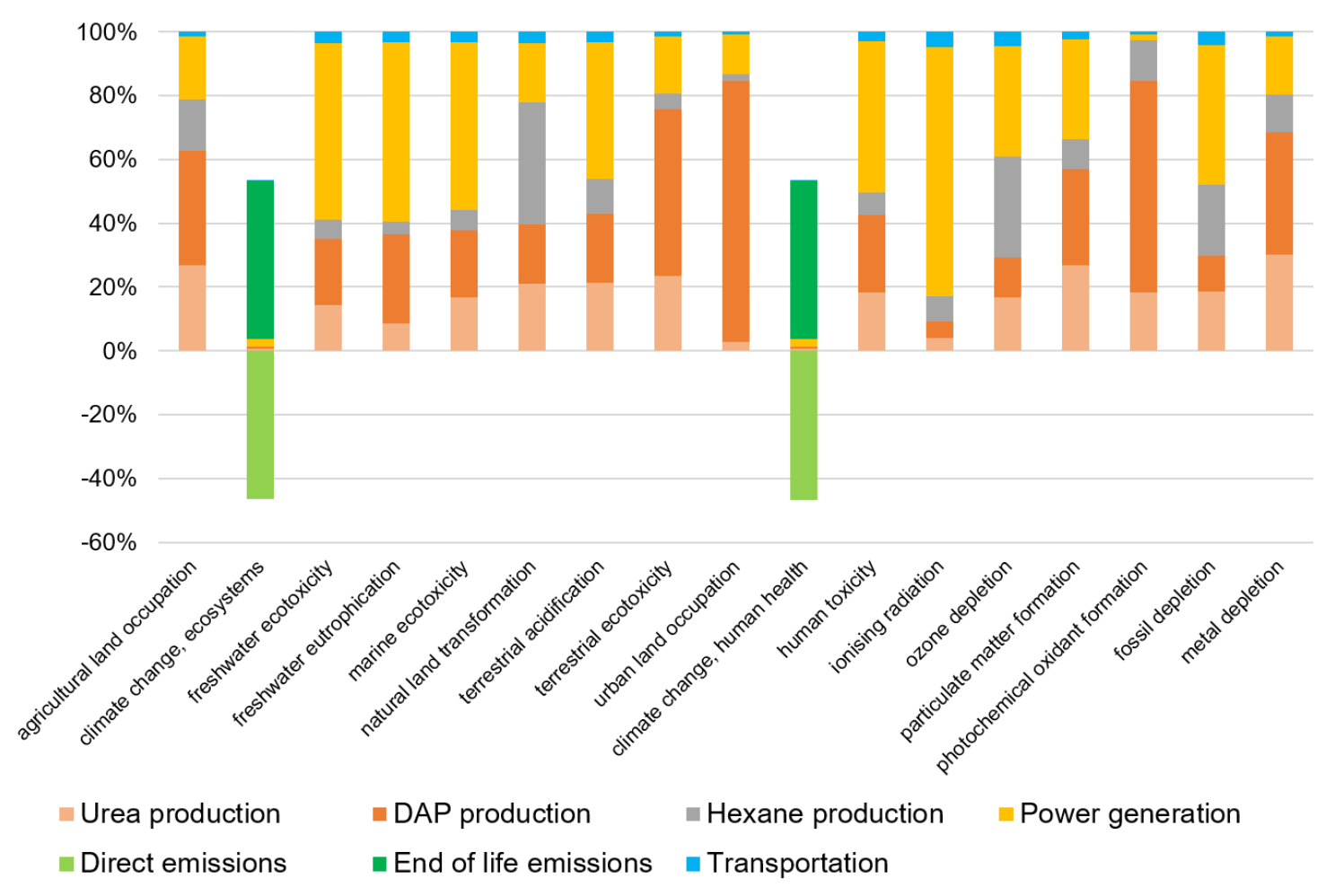

Figure 34. Environmental profile of Solution L in Figure 31.

To illuminate how the functional unit-based objective functions can influence the optimal process designs, a multiobjective optimization problem with functional unit-based environmental and economic objective functions is solved. Similar to Figure 30, the optimal solutions corresponding to the total quantity-based objective functions (shown as solid dots) lie within the feasible regions (under the Pareto optimal curves) in Figure 35. The key technologies selected by the most environmentally sustainable solution are shown in Figure 35. Hexane extraction is preferred in the lipid extraction section because the consequential environmental impacts of producing hexane are negative, reducing the total environmental 
impacts. Furthermore, the functional unit-based environmental impacts from the existing attributional LCO framework are overestimated when compared with those from the proposed consequential LCO framework.

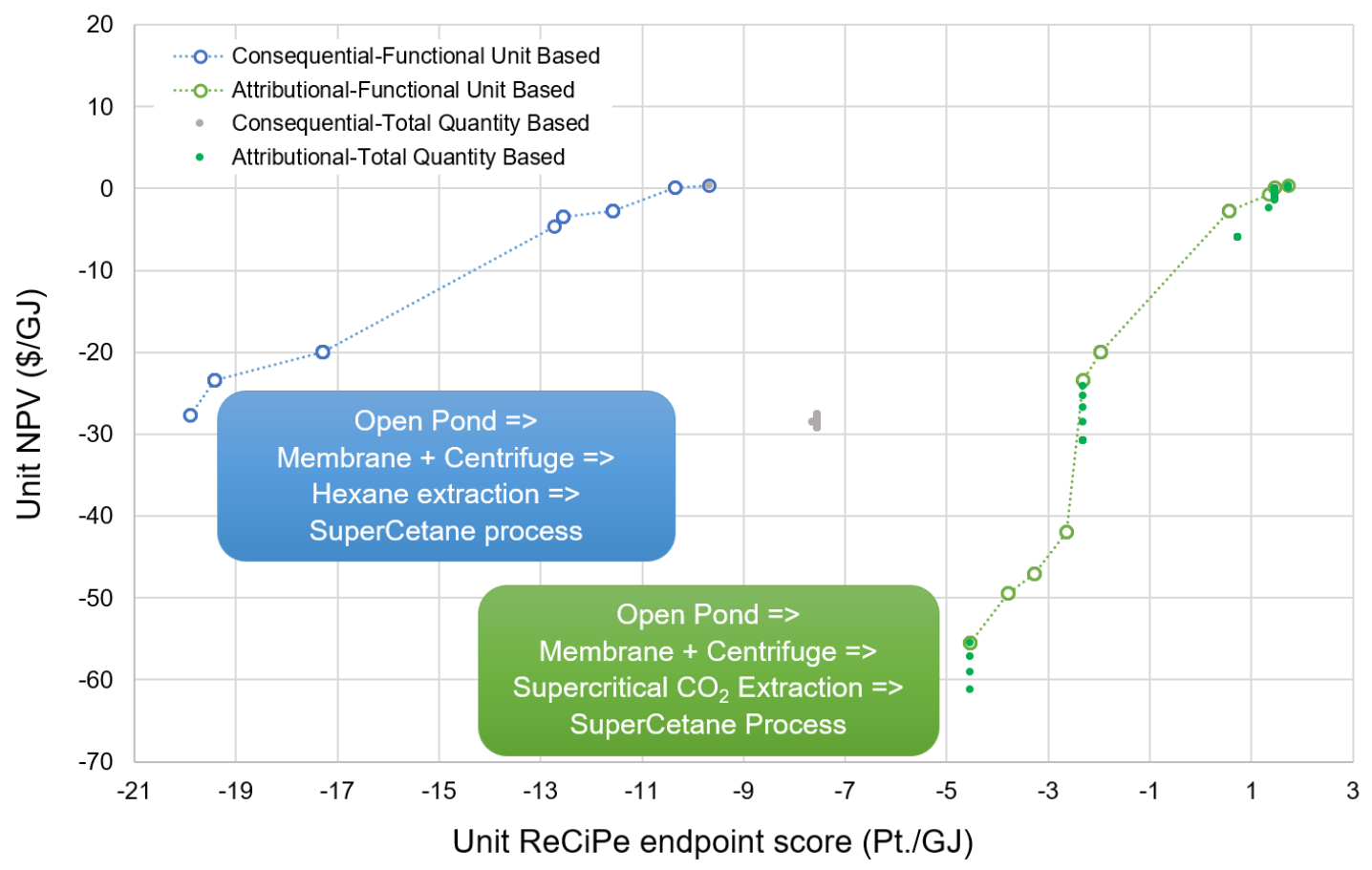

Figure 35. Pareto optimal profiles showing the trade-off between the functional unit based NPV and the functional unit based ReCiPe endpoint score. The boxes show the major process technologies for the optimal solutions with the minimum ReCiPe endpoint score per GJ of algal renewable diesel.

\subsubsection{Sensitivity analysis}

The market prices of feedstocks and products in the system are volatile compared with the process parameters which are derived from experiments. Moreover, the price elasticities of demand and supply are relatively uncertain because these parameters are estimated during specific periods and may vary over time $[247,258$, 
262, 264, 271]. In this subsection, a sensitivity analysis is conducted to investigate how the prices and price elasticities of demand and supply influence the optimization results via the consequential LCO approach. The maximum positive and negative variation are $20 \%$ and $-20 \%$ of the nominal value for each parameter.

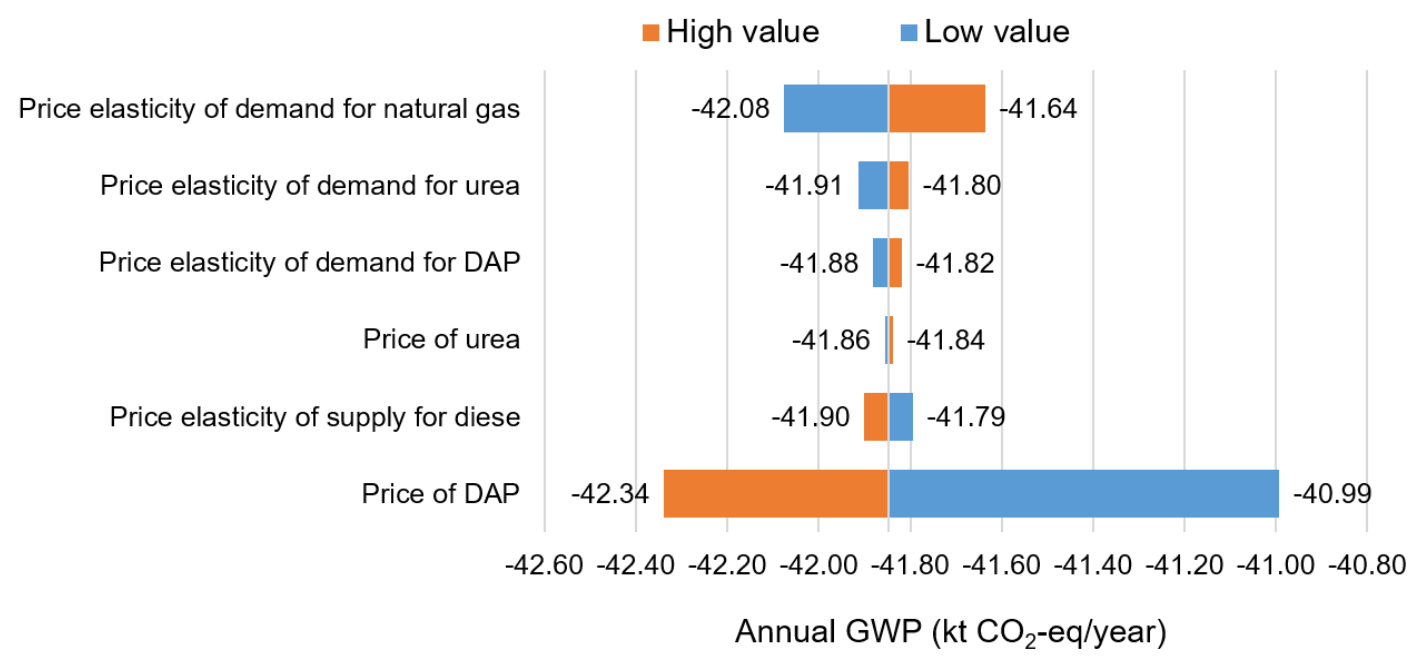

Figure 36. Sensitivity analysis for the minimum annual GWP of Solution A in Figure 27. The functional unit based GWP for Solutions A is $-89 \mathrm{~kg} \mathrm{CO}_{2}$-eq/GJ.

It is found that only prices are influential to the economic objective function, while variations in price and price elasticities can both affect the optimal environmental objective function value. For both GWP and ReCiPe endpoint score results, the variation of the price of DAP causes the most significant changes in the optimal environmental objective function value. Specifically, an increase in the price of DAP reduces the annual GWP, while increases the annual ReCiPe endpoint score. The absolute changes due to increases in parameters are more 
pronounced than those due to decreases in parameters for the annual ReCiPe endpoint score results, but is less pronounced for the GWP results. The price of diesel can dramatically influence the NPV. In all cases, the changes in NPV are positively correlated with the changes in the price of diesel. The results show that the price of diesel is critical to the profitability of the sustainable process design and a lower price of DAP is favorable in order to achieve more environmentally sustainable performance.

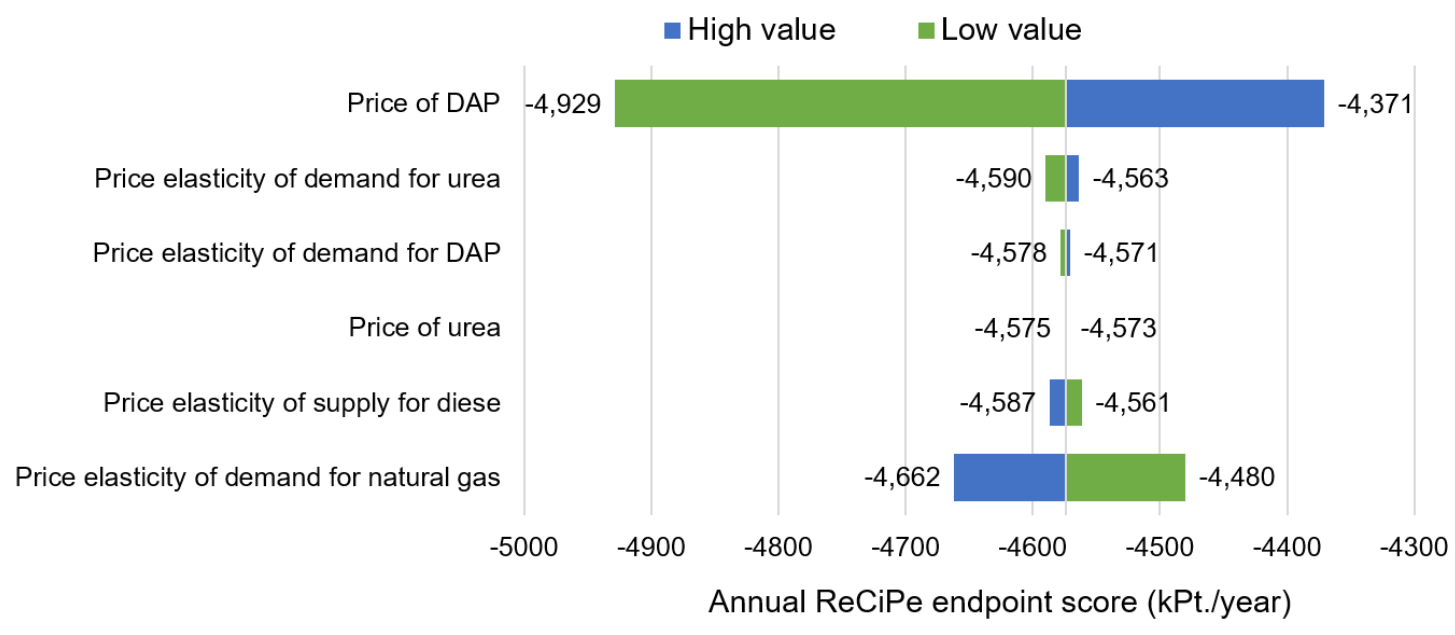

Figure 37. Sensitivity analysis for the minimum annual ReCiPe endpoint score of Solution $\mathrm{H}$ in Figure 31. The functional unit based ReCiPe endpoint score for Solutions $\mathrm{H}$ is $-9.7 \mathrm{Pt} . / \mathrm{GJ}$. 


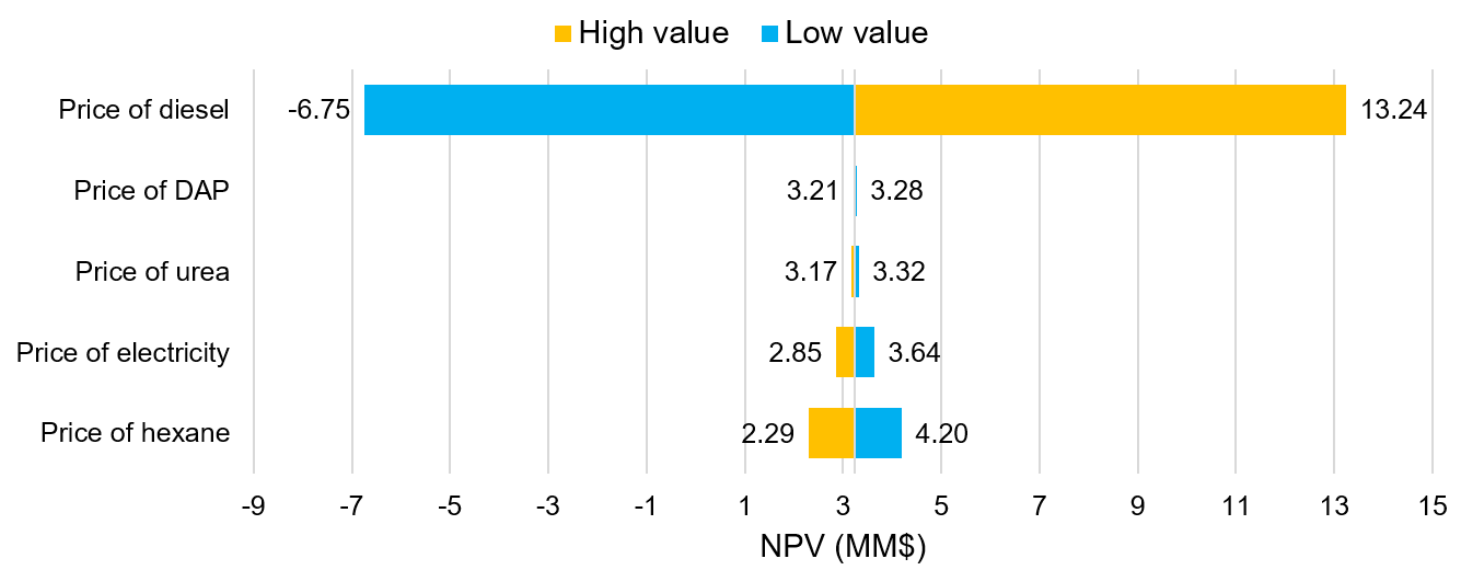

Figure 38. Sensitivity analysis for the maximum NPV of Solution A in Figure 27 and Solution $\mathrm{H}$ in Figure 31. The functional unit based NPV for Solution A is $\$ 0.4 / G J$.

\subsection{Summary}

Consequential LCA offers unique perspectives into the environmental impacts of a system by accounting for markets and their participants. However, this advantage can be overwhelmed by the difficulty in manually implementing the method for a large number of alternative systems. We addressed this challenge by developing a systematic consequential LCO framework that simultaneously determined the environmental impacts of each alternative system and automatically identified the optimal one. We developed a general system boundary that enclosed a target process and the markets and processes influenced by the target process. Based on the general system boundary, we proposed a general consequential LCO model for sustainable process design. The resulting optimization model optimized the functional unit based economic and environmental performance simultaneously 
and accounted for network configuration constraints, mass and energy balance constraints, market constraints, techno-economic evaluation constraints, and environmental impact assessment constraints. To efficiently solve the resulting nonconvex MINLP problem, we developed a tailored global optimization algorithm that integrated the inexact parametric algorithm and the branch-andrefine algorithm.

The proposed framework was applied to the sustainable process design of algal renewable diesel production. The markets for urea, DAP, natural gas, $n$-hexane, electricity, and diesel were considered for consequential life cycle optimization. We conducted a detailed market analysis to identify the consequences when establishing an algal renewable diesel production process. The most economically viable process showed a unit NPV of $\$ 0.4 / G$ J and selected open pond, settler, belt filter press, high pressure homogenization, hexane extraction, and UOP hydroprocessing from a set of alternative process designs. For this process, the minimum unit GWP values via the consequential approach and the attributional approach were -89 and $22 \mathrm{~kg} \mathrm{CO}-$ eq/GJ, respectively; the minimum unit ReCiPe endpoint scores via the consequential approach and the attributional approach were -9.7 and $1.7 \mathrm{Pt}$./GJ, respectively. The consequential approach resulted in lower environmental impacts because it accounted for sequestering the direct 
emissions in the feed gas, reducing the consumption of fossil fuels, and using environmentally friendly hexane simultaneously. The petroleum-based diesel production process was found to contribute most significantly in 10 out of 17 impact categories of the ReCiPe endpoint score via the consequential approach, but it was completely overlooked via the attributional approach. We conducted a sensitivity analysis for the identified optimal solution and found that the environmental impacts were sensitive to the price of DAP, while the NPV was significantly influenced by the renewable diesel price. The consequential LCO model could be validated by adapting the market reaction of other biofuel products in the short run and by industrial implementation results in the long run. To further reduce the environmental impacts, future development of algal renewable diesel processes could be direct to handling digestate with more efficient and environmentally sound methods.

\subsection{Appendix A: Model formulation of the consequential LCO problem}

The consequential LCO problem is formulated as a multiobjective MINFP problem. The detailed model formulation is provided as follows. 


\subsubsection{Mass balance constraints}

The mass balance relationships in the superstructure are based on a general flow relationship developed in a previous work [21, 267]. As shown in Figure 39, nine flows, namely "makeup”, “up”, "reuse”, “in”, “out”, “emission”, “down”, "product”, and "recycle", are employed to describe the mass balance relationships for technology $j$ in section $i$. There are three steps: an inlet converging step (red arrows), a conversion step (blue arrows), and an outlet separation step (green arrows).

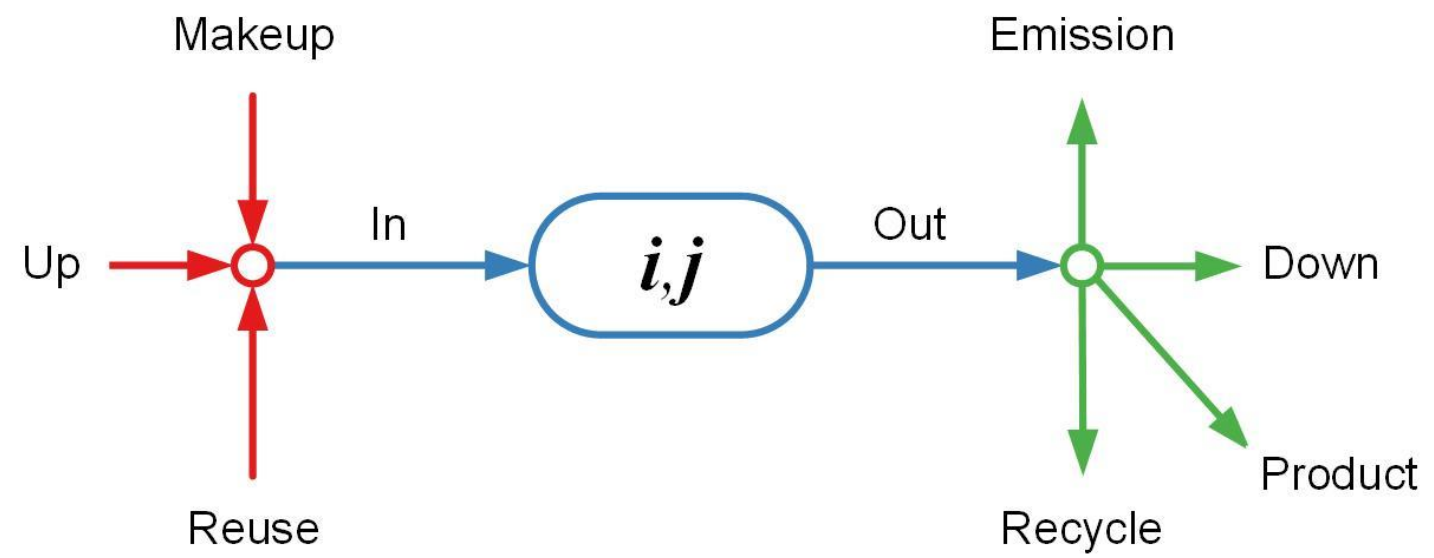

Figure 39. General flow relationships of technology $j$ in section $i$ in the mass balance [21, 267].

$$
\begin{aligned}
& 0 \leq \sum_{k \in K} M_{i, j, k}^{i \text { n }} \leq Y_{i, j} \cdot u b i_{i, j}, \quad \forall i \in I, j \in J \\
& 0 \leq M_{i, j, k}^{\text {makeup }} \leq Y_{i, j} \cdot u b m u_{i, j, k}, \quad \forall i \in I, j \in J, k \in K
\end{aligned}
$$


Constraints (1.A1) and (1.A2) are linking constraints that set upper and lower bounds of mass flow rates of the "in" and "makeup" flows of each technology using the technology selection decisions.

$$
M^{\text {feed }} \leq m^{\text {offfas }}
$$

The total feed gas flow rate is bounded above by the off-gas flow rate of upstream power plants as shown in Constraint (1.A3).

$$
\sum_{j \in J} M_{l, j, k}^{u p}=w f_{k}^{\text {feed }} \cdot M^{\text {feed }}, \quad \forall k \in K
$$

In the first section, the mass flow rate of each species of the "up" flow is defined using the total feed gas mass flow rate and its composition as shown Eq. (1.A4).

$$
\sum_{j \in J} M_{i, j, k}^{u p}=\sum_{j \in J} M_{i-1, j, k}^{d o w n}, \quad \forall 2 \leq i \leq 7, k \in K
$$

In Sections 2-7, the mass flow rates of the "up" flows are equal to the mass flow rates of the "down" flows in previous sections as shown in Eq. (1.11).

$$
\sum_{j \in J} M_{8, j, k}^{u p}=\sum_{j \in J}\left(M_{7, j, k}^{\text {down }}+M_{l o, j, k}^{\text {down }}\right), \quad \forall k \in K
$$

In section 8, the "up" flow mixes the "down" flows from both sections 7 and 10 as shown in Eq. (1.A6).

$$
M_{9, j, k}^{u p}=0, \quad \forall j \in J, k \in K
$$

The feedstock of anaerobic digestion comes from recycling the remnant of the lipid extraction section and makeup water. Therefore, the mass flow rates of the "up" flow of section 9 are fixed to be 0 as shown in Eq. (1.A7). 


$$
\sum_{j \in J} M_{10, j, k}^{u p} \leq \sum_{j \in J} M_{9, j, k}^{d o w n}, \quad \forall k \in K
$$

The biogas product stream from the anaerobic digestion process, or the "down" flow of section 9, is split and sent to different sections. Depending on the amount of hydrogen needed, a portion of the biogas is sent to section 10 for hydrogen production, while the remaining is used for utility generation. Therefore, the mass flow rate of the "up" flow of section 10 is smaller or equal to the mass flow rate of the "down" flow of section 9 as shown in Eq. (1.A8).

$$
\sum_{j \in J} M_{l 1, j, k}^{u p}=\sum_{j \in J}\left(M_{8, j, k}^{\text {down }}+M_{9, j, k}^{\text {down }}-M_{l 0, j, k}^{u p}\right), \quad \forall k \in K
$$

Accordingly, the "up" flow of section 11 collects the fuel gas from section 8 and the remaining biogas from section 9 as shown in Eq. (1.A9).

$$
\begin{aligned}
& \sum_{j \in J} M_{I, j, k}^{\text {reuse }}=\sum_{i^{\prime} \in\{2,3,4,5,8,9,10,11\}, j \in J} M_{i^{\prime}, j, k}^{\text {recycle }}, \quad \forall 1 \leq k \leq 11 \cup 15 \leq k \leq 19 \\
& \sum_{j \in J} M_{1, j, k}^{\text {reuse }}=d n \cdot \sum_{i^{\prime} \in\{2,3,4,5,8,9,10,11\}, j \in J} M_{i^{\prime}, j, k}^{\text {recycle }}, \quad \forall 12 \leq k \leq 14
\end{aligned}
$$

As shown in Eqs. (1.A10) and (1.A11), the cultivation section reuses several "recycle" flows in downstream sections. It is noted that the cultivation section is the only section that ceases operations at night. The recycling gas is emitted at night, while the recycling solids including the algae biomass and nutrients remain in the cultivation section. The operation during daytime is explicitly modeled in the mass balance constraints and modifications are made to reflect the operations 
at night. Therefore, the reused gas and water during daytime are exactly the "recycle" flows generated during daytime in Eq. (1.A10), while the "reused" solids during daytime account for accumulation at night in Eq. (1.A11).

$$
\begin{aligned}
& \sum_{j \in J} M_{9, j, k}^{\text {reuse }}=\sum_{j \in J} M_{7, j, k}^{\text {recycle }}, \quad \forall k \in K \\
& M_{i, j, k}^{\text {reuse }}=0, \quad \forall 2 \leq i \leq 8 \cup 10 \leq i \leq 11, j \in J, k \in K
\end{aligned}
$$

As in Eq. (1.A12), the anaerobic digestion section reuses the "recycle" flows from the lipid extraction section. The other sections do not reuse flows as described in Eq. (1.A13).

$$
M_{i, j, k}^{i n}=M_{i, j, k}^{u p}+M_{i, j, k}^{\text {reuse }}+M_{i, j, k}^{\text {makeup }}, \quad \forall i \in I, j \in J, k \in K
$$

The inlet converging step is modeled by Eq. (1.A14), where the "in" flow of technology $j$ in section $i$ combines the "up", "reuse", and "makeup" flows sent to the same technology.

$$
M_{i, j, k}^{i n}=\sum_{k^{\prime} \in K}\left(p_{i, j, k, k^{\prime}}^{i n} \cdot M_{i, j, k^{\prime}}^{i n}\right), \quad \forall i \in I, j \in J, k \in K
$$

The flow rates of various species in the "in" flows are proportional to the key components in the "in" flows according to stoichiometry relationships of reactions. The relationships are modeled using parameter $p_{i, j, k, k^{\prime}}^{i n}$ in Eq. (1.A15).

$$
M_{i, j, k}^{\text {out }}=M_{i, j, k}^{\text {in }}+\sum_{k^{\prime} \in K}\left(s c_{i, j, k, k^{\prime}} \cdot x_{i, j} \cdot M_{i, j, k^{\prime}}^{i n}\right), \quad \forall i \in I, j \in J, k \in K
$$


The conversion step of each technology is modeled using Eq. (1.A16), where $s c_{i, j, k, k^{\prime}}$ denotes the stoichiometric coefficient and $\boldsymbol{x}_{i, j}$ denotes the conversion.

$$
M_{i, j, k}^{\text {out }}=M_{i, j, k}^{\text {down }}+M_{i, j, k}^{\text {recycle }}+M_{i, j, k}^{\text {emission }}+M_{i, j, k}^{\text {product }}, \quad \forall i \in I, j \in J, k \in K
$$

The "out" flow of a section is split into the "down", "recycle", "emission", and "product" flows as shown in Eq. (1.A17).

$$
M_{i, j, k}^{\text {down }}=\sum_{k^{\prime} \in K}\left(s f_{i, j, k, k^{\prime}}^{\text {down }} \cdot M_{i, j, k^{\prime}}^{\text {out }}\right), \quad \forall i \in I, j \in J, k \in K
$$

In Eq. (1.A18), the "down" flow comes from the "out" flow based on split fraction $s f_{i, j, k, k^{\prime}}^{\text {down }}$

$$
M_{i, j, k}^{\text {emission }}=\sum_{k^{\prime} \in K}\left(s f_{i, j, k, k^{\prime}}^{\text {emissionl }} \cdot M_{i, j, k^{\prime}}^{\text {out }}\right)-s f_{i, j, k}^{\text {emission2 }} \cdot M_{i, j, k}^{\text {down }}, \quad \forall i \in I, j \in J, k \in K
$$

The flow rates of the "emission" flows depend on both the flow rates of the "out" and "down" flows as described in Eq. (1.A19).

$$
M_{i, j, k}^{\text {product }}=\sum_{k^{\prime} \in K}\left(s f_{i, j, k, k^{\prime}}^{\text {product }} \cdot M_{i, j, k^{\prime}}^{\text {out }}\right), \quad \forall i \in I, j \in J, k \in K
$$

Similar to the "down" flow, the "product" flow is directly split from the "out" flow as shown in Eq. (1.A20). The remaining materials in the "out" flows are sent to the corresponding "recycle" flows.

$$
T E=u c \cdot \sum_{j \in J, k \in K} M_{8, j, k}^{\text {product }}
$$

The energy content of the renewable diesel is evaluated as in Eq. (1.A21), where uc is the unit converter. 


\subsubsection{Energy balance constraints}

$$
H C_{i, j}=\sum_{k \in K}\left(u h c o_{i, j, k} \cdot M_{i, j, k}^{\text {out }}+u h c i_{i, j, k} \cdot M_{i, j, k}^{i n}\right), \quad \forall i \in I, j \in J
$$

The heat consumption of technology $j$ in section $i$ is calculated using Eq. (1.A22), where parameters $u h c o_{i, j, k}$ and $u h c i_{i, j, k}$ are unit heat consumption based on the "out" and "in" flows, respectively.

$$
H G=\eta^{H R S G} \cdot\left(1-\eta^{G T}\right) \cdot \sum_{k \in K}\left(l h v_{k} \cdot M_{I 1, l, k}^{i n}\right)
$$

The heat generation rate is calculated using lower heating values of the fuel gas entering the gas turbine. In Eq. (1.A23), $\eta^{H R S G}, \eta^{G T}$, and $l h v_{k}$ are heat generation efficiencies of the heat recovery steam generation system, power generation efficiency of the gas turbine, and lower heating value of species $k$, respectively.

$$
P C^{\text {feed }}=\frac{u p c^{\text {feed }}}{d n} \sum_{j \in J} M_{l, j, 2}^{u p}
$$

Eq. (1.A24) calculates the power consumption of transporting feed gas. up $c^{\text {feed }}$ is the power consumption rate for transporting 1 tonne $\mathrm{CO}_{2}$ in the feed gas.

$$
P C^{\text {cooling }}=u p c^{\text {cooling }} \cdot\left(H G-\sum_{i \in I, j \in J} H C_{i, j}\right)
$$

Eq. (1.A25) calculates the power consumption for cooling extra heat generated. Parameter upcooling is the power consumption rate for cooling $1 \mathrm{MW}$ heat.

$$
P C_{i, j}=\sum_{k \in K}\left(u_{p c o} o_{i, j, k} \cdot M_{i, j, k}^{\text {out }}+u p c i_{i, j, k} \cdot M_{i, j, k}^{i n}\right), \quad \forall i \in I, j \in J
$$


The power consumption of technology $j$ in section $i$ is calculated using Eq. (1.A26), where parameters $u p c o_{i j, k}$ and $u h c i_{i j, k}$ are unit power consumption rates based on the "out" and "in" flows, respectively.

$$
P G=\eta^{G T} \cdot \sum_{k \in K}\left(l h v_{k} \cdot M_{l l, l, k}^{i n}\right)
$$

The power generation rate $P G$ is calculated by Eq. (1.A27).

$$
P E-P I=P G-\sum_{i \in I, j \in J} P C_{i, j}-P C^{\text {cooling }}-P C^{\text {feed }}
$$

The power balance is enforced by Eq. (1.A28), where $P E$ and $P I$ are power export and import rate, respectively.

\subsubsection{Market constraints}

$$
\begin{aligned}
& A S_{l}=\sum_{p \in P P C_{l}}\left(a s c_{l, p} \cdot X S_{l, p}+b s c_{l, p} \cdot Y S_{l, p}\right)+P S_{l}, \quad \forall l \in\{1,2,4\} \\
& a s c_{l, p}=\frac{n s_{l, p}-n s_{l, p-1}}{m s_{l, p}-m s_{l, p-1}}, \quad \forall l \in\{1,2,4\}, p \in P P C_{l} \\
& b s c_{l, p}=n s_{l, p}-a s c_{l, p} \cdot m s_{l, p}, \quad \forall l \in\{1,2,4\}, p \in P P C_{l} \\
& P S_{4}=P E \\
& P S_{l}=0, \quad \forall l \in\{1,2\} \\
& \sum_{p \in P P C_{l}} Y S_{l, p}=1, \quad \forall l \in\{1,2,4\} \\
& \sum_{p \in P P C_{l}} X S_{l, p}=P R_{l}, \quad \forall l \in\{1,2,4\} \\
& m s_{l, p} \cdot Y S_{l, p-1} \leq X S_{l, p} \leq m s_{l, p} \cdot Y S_{l, p}, \quad \forall l \in\{1,2,4\}, p \in P P C_{l}
\end{aligned}
$$


Eqs. (1.A29)-(1.A31) define the aggregate supply curves of markets 1, 2, and 4 using piecewise linear functions. Parameter $n S l, p$ is the aggregate supply in market $l$ at partition point $p$ and $m S_{l, p}$ is the price in market $l$ at partition point $p$. Continuous variables $A S_{l}$ is the aggregate supply in market $l, P R_{l}$ is the price in market $l$, and $X S_{l, p}$ is the price in market $l$ in interval $p$. Integer variable $Y S_{l, p}$ is introduced to model the selection of partition interval $p$.

$$
A S_{l}=\frac{e s_{l} \cdot \alpha_{l}}{\beta_{l}} \cdot P R_{l}+\alpha_{l} \cdot\left(1-e s_{l}\right), \quad \forall l \in\{3,5\}
$$

The aggregate supplies of natural gas and diesel are modeled by Eq. (1.A37), where $e s l$ is the price elasticity of supply, $\alpha_{l}$ and $\beta_{l}$ are the original aggregate supply and the original price in the market, respectively.

$$
\begin{aligned}
& A D_{l}=\frac{e d_{l} \cdot \alpha_{l}}{\beta_{l}} \cdot P R_{l}+\alpha_{l} \cdot\left(1-e d_{l}\right)+P D_{l}, \quad \forall l \in L \\
& P D_{l}=\sum_{i \in l, j \in J}\left(\frac{M_{i, j, l 2}^{\text {makeup }}}{d n}-M_{i, j, 12}^{\text {product }}\right) \\
& P D_{2}=\sum_{i \in l, j \in J}\left(\frac{M_{i, j, 13}^{\text {makeup }}}{d n}-M_{i, j, 13}^{\text {product }}\right) \\
& P D_{3}=\sum_{i \in l, j \in J} M_{i, j, 7}^{\text {makeup }} \\
& P D_{4}=P I \\
& P D_{5}=-\sum_{j \in J, k \in K} M_{8, j, k}^{\text {product }}
\end{aligned}
$$




$$
P D_{6}=\sum_{i \in I, j \in J} M_{i, j, 18}^{\text {makeup }}
$$

Eq. (1.A38) defines the aggregate demand curve in the market $l$ based on the definition of price elasticity of demand edl. Parameters $\alpha_{l}$ and $\beta_{l}$ are the original aggregate demand and the original price in market $l$, respectively. Variables $A D_{l}$ and $P D_{l}$ are the aggregate demand and process demand in the market 1 , respectively. The specific forms of $P D_{l}$ are defined in Eqs. (1.A39)-(1.A44).

$$
A S_{l}=A D_{l}, \quad \forall l \in L
$$

A new market equilibrium after the deployment of the algae process is enforced by Eq. (1.A45).

\subsubsection{Techno-economic analysis constraints}

$$
\begin{aligned}
D C_{i, j}=c c i b_{i, j} & \cdot\left(\frac{\sum_{k \in K I_{i, j}} M_{i, j, k}^{i n}}{m i b_{i, j}}\right)^{s c_{i, j}} \cdot \frac{\text { cepcir }}{\text { cepcib }_{i, j}} \\
& +\operatorname{ccob}_{i, j} \cdot\left(\frac{\sum_{k \in K o_{i, j}} M_{i, j, k}^{\text {out }}}{\text { mob }_{i, j}}\right)^{s c f_{i, j}} \cdot \frac{\text { cepcir }}{\text { cepcib }_{i, j}}, \forall i, j
\end{aligned}
$$

The direct cost of each technology is evaluated using nonlinear function as shown in Eq. (1.23), where $c c i b_{i, j}$ and $c c o b_{i, j}$ are the base-case capital costs with respect to base-case "in" flow $m i b_{i, j}$ aand "out" flow $m o b_{i, j}$, respectively. $s c f_{i, j}$ is the scaling factor of technology/process $j$ in section $i$. cepcir and cepcibi,j are the chemical 
engineering plant cost index in the reference year and in the base-case year for technology/process $j$ in section $i$, respectively. Since the capital cost data for different technologies are based on input or output mass flow rates, both types of data are considered in the model.

$$
F C I=f c i c \cdot \sum_{i \in I, j \in J} D C_{i, j}
$$

Fixed capital investment $F C I$ is calculated based on the direct cost of each technology as shown in Eq. (1.A47).

$$
\begin{aligned}
& \text { all } \leq a c \cdot \sum_{j \in J} \frac{M_{l, j, 14}^{\text {out }}-M_{l, j, 14}^{\text {in }}}{\text { productivity }_{j}} \leq a u l \\
& L C=l p \cdot a c \cdot \sum_{j \in J} \frac{M_{l, j, 14}^{\text {out }}-M_{l, j, 14}^{\text {in }}}{\text { productivity }_{j}}
\end{aligned}
$$

The area limit is enforced by Eq. (1.A48), where $a c$ is the coefficient to account for total land area of the facility, productivity $y_{j}$ is area productivity of cultivation system $j$, all is the area lower limit, and aul is the area upper limit. Land cost is calculated in Eq. (1.A49).

$$
W O C=w c c \cdot F C I
$$

Working capital WOC is calculated as a product of the fixed capital investment and working capital coefficient wcc as shown in Eq. (1.A50).

$$
T C I=F C I+L C+W O C
$$


Total capital investment $T C I$ is calculated as the sum of fixed capital investment, land cost, and working capital as shown in Eq. (1.A50).

$$
\begin{aligned}
F C= & h \cdot\left(P R_{1}+\text { retail }_{1}\right) \cdot P D_{1}+h \cdot\left(P R_{2}+\text { retail }_{2}\right) \cdot P D_{2} \\
& +h \cdot P R_{3} \cdot P D_{3}+h \cdot \beta_{6} \cdot P D_{6}
\end{aligned}
$$

Feedstock cost is calculated using Eq. (1.A52), where $h$ is the total hours in a year and retailı represents the differences between market price and retail price.

$$
U C=h \cdot P R_{4} \cdot P I
$$

Utility cost is calculated using Eq. (1.A53).

$$
O C=\sum_{j \in J,} M_{5, j, 14}^{u p} / b m r \cdot o c r
$$

Operations cost is calculated using Eq. (1.A54), where $b m r$ is the reference biomass generation rate and ocr is the reference operations cost.

$$
M C=\sum_{i \in I, j \in J} m c c_{i, j} \cdot D C_{i, j}
$$

Maintenance cost of each technology is a percentage of the direct cost as shown in Eq. (1.A55).

$$
P T I=p t i c \cdot F C I
$$

Property tax and insurance is calculated as a percentage of fixed capital investment as shown in Eq. (1.A56).

$$
D P C=d p c c \cdot F C I
$$

Depreciation cost is calculated as a percentage of fixed capital investment as shown in Eq. (1.A57). 


$$
S=h \cdot\left(-P R_{5} \cdot P D_{5}+P R_{4} \cdot P E\right)
$$

The total sales include the revenue from selling renewable diesel and extra power as shown in Eq. (1.A58).

$$
G E=g e c \cdot S
$$

General expense is calculated as a percentage of sales as shown in Eq. (1.A59).

$$
\begin{aligned}
& I T \geq \operatorname{tr} \cdot(S-F C-U C-O C-M C-G E-P T I-D C) \\
& I T \geq 0 \\
& I T C=-h \cdot \text { credit } \cdot P D_{5}
\end{aligned}
$$

Income tax is calculated as a percentage of sales less several costs as in Eq. (1.A60). By enforcing Eq. (1.A61), the income tax is not levied over a project with a negative income. The income tax credit is calculated as shown in Eq. (1.A62).

$$
A C F=S-F C-U C-O C-M C-G E-P T I-I T+I T C
$$

The annual cash flow is calculated in Eq. (1.A63).

$$
O B J_{\text {economic }}=\frac{\frac{i r \cdot\left(1-i r^{l s}\right)}{1-i r} \cdot A C F-T C I}{l s \cdot h \cdot T E}
$$

In Eq. (1.A64), the functional unit based net present value is calculated as the ratio of NPV to the total energy of the renewable diesel over the project lifetime. ir is the interest rate and $l s$ is the project lifetime. 


\subsubsection{Environmental impact evaluation constraints}

$$
P S C_{l}=A S_{l}-P S_{l}-\alpha_{l}, \quad \forall l \in L
$$

For the suppliers' processes in market 1 , the changed amount is calculated by Eq. (1.A65).

$$
\operatorname{EICS}_{l, t}=\operatorname{ccfsup}_{l, t} \cdot P S C_{l}, \quad \forall l \in L, t \in T
$$

Therefore, the environmental impact associated with the change of supply is evaluated by Eq. (1.A66). ccfsupl,t is the consequential characterization factor of supplying $l$ in impact category $t$ as evaluated by Ecoinvent.

$$
C D C_{l}=A D_{l}-P D_{l}-\alpha_{l}, \quad \forall l \in L
$$

Eq. (1.A67) calculates the change in the demand of other customers in market 1 .

$$
C P C_{l, s}=\text { yield }_{l, s} \cdot c a_{l, s} \cdot C D C_{l}, \quad \forall l \in L
$$

Next, the consequences of the change in the other customers' demand are evaluated. The potential products of the other customers' processes are indexed by s. As a change in 1 may influence multiple products, parameter cal,s is introduced to allocate the change in the demand of $l$ to a specific process for generating $s$. The yield $d_{l, s}$ is introduced to translate the change in $l$ to the change in $s$.

$$
E I C D_{l, t}=\sum_{s \in S}\left[\left(c c f c u s_{s, t}-c c f s u b_{s, t}\right) \cdot C P C_{l, s}\right]-c f_{l} \cdot c c f s u p_{l, t} \cdot C D C_{l}, \forall l, t
$$

The environmental impact associated with the change in the other customers' demand is evaluated in Eq. (1.A69). $c c f c u s_{s, t}$ and $c c f s u b_{s, t}$ are the consequential 
characterization factors of producing $s$ in impact category $t$ by the customers and substitute processes, respectively. For feedstock markets, $C P C_{l, s}$ is negative, so the first summation in Eq. (1.A69) indicates that the changed amount of $l$ is no long produced. In fact, feedstock $l$ is still manufactured, but it is transferred to the renewable diesel production process. Such an effect does not apply to product markets, or when $C P C_{l, s}$ is positive. Therefore, the environmental impact is modified by adding back the environmental impact associated with producing feedstock 1 .

$$
E O L E_{t}^{\text {digestate }}=c c f_{t}^{C} \cdot M_{9,1,17}^{\text {product }}+c c f_{t}^{N} \cdot M_{9,1,12}^{\text {product }}, \quad \forall t \in T
$$

Another modification is made in Eq. (1.A70), because the digestate is sold as a nitrogen fertilizer, which will incur additional carbon dioxide and nitrous oxide emissions during the use of the fertilizer [159].

$$
\begin{aligned}
D E_{k}=( & \left.\sum_{j \in J} M_{l, j, k}^{\text {emission }}+\operatorname{gas}_{k} \cdot \sum_{j \in J} M_{l, j, k}^{\text {reuse }}-\sum_{j \in J} M_{l, j, k}^{u p}\right) / d n \\
& +\sum_{i \geq 2, j \in J} M_{i, j, k}^{\text {emission }}, \quad \forall k \in K
\end{aligned}
$$

The net direct emissions from the renewable diesel production process are evaluated by Eq. (1.A71). The first term and the second term account for the emissions from the cultivation section during daytime and nighttime operation, respectively. gask is the gas species in the set $\mathrm{K}$ to be emitted to the atmosphere. 
The third term accounts for the amount of the off-gas absorbed in the feed during daytime operating. The last term accounts for the emissions from other sections.

$$
E I D E_{t}=\sum_{k \in K} c c f_{k, t} \cdot D E_{k}, \quad \forall t \in T
$$

The environmental impact associated with the direct emissions is evaluated in Eq. (1.A72).

$$
\begin{aligned}
& \operatorname{TRANSE}_{l, t}=\left(\operatorname{ccfsup}_{5, t}+\operatorname{eolcf}_{5, t}\right) \cdot \text { fuel }_{1} \cdot \sum_{i \in l, j \in J}\left(M_{i, j, 12}^{\text {makeup }} / d n+M_{i, j, 12}^{\text {product }}\right), \forall t \\
& \operatorname{TRANSE}_{2, t}=\left(\operatorname{ccfsup}_{5, t}+\operatorname{eolcf}_{5, t}\right) \cdot \text { fuel }_{2} \cdot \sum_{i \in I, j \in J}\left(M_{i, j, 13}^{\text {makeup }} / d n+M_{i, j, 13}^{\text {product }}\right), \forall t \\
& \operatorname{TRANSE}_{3, t}=\left(\operatorname{ccfsup}_{3, t}+\operatorname{eolcf}_{3, t}\right) \cdot \text { fuel }_{3} \cdot \sum_{i \in I, j \in J} M_{i, j, 7}^{\text {makeup }} \\
& +c c f_{7, t} \cdot \text { fugitive } \cdot \sum_{i \in I, j \in J} M_{i, j, 7}^{\text {makeup }}, \forall t \\
& \operatorname{TRANSE}_{4, t}=\operatorname{ccfsup}_{4, t} \cdot \text { fuel }_{4} \cdot(P I+P E), \quad \forall t \in T \\
& \operatorname{TRANSE}_{5, t}=\left(\operatorname{ccfsup}_{5, t}+\operatorname{eolcf}_{5, t}\right) \cdot \text { fuel }_{5} \cdot \sum_{j \in J, k \in K} M_{8, j, k}^{\text {product }}, \quad \forall t \in T \\
& \text { TRANSE }_{6, t}=\left(\operatorname{ccfsup}_{5, t}+\text { eolcf }_{5, t}\right) \cdot \text { fuel }_{\sigma} \cdot \sum_{i \in I, j \in J} M_{i, j, 18}^{\text {makeup }}, \quad \forall t \in T
\end{aligned}
$$

The environmental impacts associated with transportation are evaluated by Eqs. (1.A73)-(1.A78)

$$
\begin{aligned}
& T E I C=\sum_{l \in L, t \in T}\left(E I C S_{l, t}+E I C D_{l, t}+E O L E_{t}^{\text {digestate }}+E I D E_{t}+T R A N S E_{l, t}\right) \\
& O B J_{\text {environmental }}=\frac{T E I C}{T E}
\end{aligned}
$$


The total environmental impact change TEIC sums up the environmental impacts associated with different life cycle stages in Eq. (1.A79). The functional unit based environmental impact change is evaluated using Eq. (1.A80).

\subsubsection{Superstructure network configuration constraints}

$$
\begin{aligned}
& \sum_{j \in J} Y_{i, j}=1, \quad \forall i \in I \\
& Y_{3,1}=Y_{4,1} \\
& Y_{5,2}+Y_{5,3}=Y_{6,6} \\
& Y_{6,6}=Y_{7,3}+Y_{7,4}
\end{aligned}
$$

Binary variable $Y_{i, j}$ is introduced to select technology $j$ in section $i . Y_{i, j}$ is equal to 1 if the corresponding technology is selected, and 0 otherwise. Eq. (1.A81) requires that one and only one technology is selected in each section in the network. The technologies indexed by $\langle i, j>$ are $<1,1>$ open pond, $<1,2>$ flat plate $\mathrm{PBR},<1,3>$ bubble column PBR, $<1,4>$ tubular PBR, $<2,1>$ settler, $<3,1>$ blank, $<3,2>$ hollow fiber membrane, $<4,1>$ belt filter press, $<4,2>$ centrifuge, $<5,1>$ blank, $<5,2>$ freeze drying, $<5,3>$ thermal drying, $<6,1>$ wet bead beating, $<6,2>$ microwaving, $<6,3>$ high pressure homogenization, $\langle 6,4\rangle$ sonication, $\langle 6,5\rangle$ blank, $\langle 6,6\rangle$ dry bead beating, $<7,1>$ wet hexane extraction, $<7,2>$ wet supercritical $\mathrm{CO}_{2}$ extraction, $<7,3>$ dry hexane extraction, $<7,4>$ dry supercritical $\mathrm{CO}_{2}$ extraction, $<8,1>$ UOP hydroprocessing, $<8,2>$ SuperCetane hydroprocessing, $<9,1>$ anaerobic digestion, 
$<10,1>$ hydrogen generation, and $<11,1>$ utility generation. Since belt filter presses can reduce the water content from $1 \%$ to $20 \%$ directly, a primary dewatering process will be bypassed if belt filter press dewatering is selected. This relationship is enforced by Eq. (1.A82). The capital investment and energy consumption of disrupting dried biomass differ from those for wet biomass. As a result, the products of drying technologies are disrupted by a tailored technology as described by Eq. (1.A83). Moreover, by enforcing Eq. (1.A84), the dried and disrupted algae biomass is sent to lipid extraction processes that operate under different operating conditions from those for wet algae biomass.

\subsection{Appendix B: Model Formulation of the Auxiliary Parametric}

\section{Problem}

$$
\begin{aligned}
& \sum_{k \in K I_{i, j}} M_{i, j, k}^{\text {in }}+\sum_{k \in K O_{i, j}} M_{i, j, k}^{\text {out }}=\sum_{n=1}^{N}\left(W C_{i, j, n} \cdot u c_{i, j, n}\right), \quad \forall i \in I, j \in J \\
& \sum_{n=1}^{N} W C_{i, j, n}=1, \quad \forall i \in I, j \in J \\
& \sum_{n=1}^{N-1} E C_{i, j, n}=1, \quad \forall i \in I, j \in J \\
& W C_{i, j, 1} \leq E C_{i, j, 1}, \quad \forall i \in I, j \in J \\
& W C_{i, j, 1} \leq E C_{i, j, n-1}+E C_{i, j, n}, \quad \forall i \in I, j \in J, 2 \leq n \leq N-1 \\
& W C_{i, j, N} \leq E C_{i, j, N-1}, \quad \forall i \in I, j \in J
\end{aligned}
$$




$$
\begin{aligned}
& 0 \leq W C_{i, j, n} \leq 1, \quad \forall i \in I, j \in J, n \in N \\
& D C_{i, j}=\sum_{n=1}^{N}\left(W C_{i, j, n} \cdot c c_{i, j, n}\right), \quad \forall i \in I, j \in J
\end{aligned}
$$

To handle the separable concave terms for calculating the CAPEX, we approximate the original nonlinear functions in Eq. (1.23) by successive piecewise linear approximation functions in Eqs. (1.B1)-(1.B8). A set of SOS1 variables $E C_{i, j, n}$ are used in the piecewise linear approximation functions, so that at most one variable over set $N$ can be nonzero.

$$
\begin{aligned}
& X B_{m}=\sum_{n=1}^{N}\left(W B_{m, n} \cdot u b_{m, n}\right), \quad \forall m \in M \\
& \sum_{n=1}^{N} W B_{m, n}=1, \quad \forall m \in M \\
& \sum_{n=1}^{N-1} E B_{m, n}=1, \quad \forall m \in M \\
& W B_{m, 1} \leq E B_{m, 1}, \quad \forall m \in M \\
& W B_{m, 1} \leq E B_{m, n-1}+E B_{m, n}, \quad \forall m \in M, 2 \leq n \leq N-1 \\
& W B_{m, N} \leq E B_{m, N-1}, \quad \forall m \in M \\
& 0 \leq W B_{m, n} \leq 1, \quad \forall m \in M, n \in N \\
& B T_{p}=\sum_{\left(m, m^{\prime}\right) \in M B_{p}, n \in N, n^{\prime} \in N}\left(u b_{m, n} \cdot u b_{m^{\prime}, n^{\prime}} \cdot K T_{p, n, n^{\prime}}\right), \quad \forall p \in P \\
& \sum_{n^{\prime} \in N} K T_{p, n, n^{\prime}}=\sum_{m \in M B_{p}} W B_{m, n}, \quad \forall p \in P, n \in N \\
& \sum_{n \in N} K T_{p, n, n^{\prime}}=\sum_{m \prime^{\prime} \in M B_{p}} W B_{m^{\prime}, n^{\prime}}, \quad \forall p \in P, n^{\prime} \in N
\end{aligned}
$$




$$
\begin{aligned}
& F C=h \cdot\left[B T_{1}+\text { retail }_{1} \cdot P D_{1}+B T_{2}+\text { retail }_{2} \cdot P D_{2}+B T_{3}+\beta_{6} \cdot P D_{6}\right] \\
& U C=h \cdot B T_{4} \\
& S=h \cdot\left(B T_{5}+B T_{6}\right)
\end{aligned}
$$

An auxiliary variable $X B_{m}$ is introduced for the bilinear terms. $X B_{1}-X B_{5}$ are equal to $P R_{1}-P R_{5}$, respectively; $X B_{6}-X B_{8}$ are equal to $P D_{1}-P D_{3}$, respectively; $X B_{9}$ is equal to $-P D_{5}, X B_{10}$ is equal to $P I ; X B_{11}$ is equal to $P E$. The continuous variables are partitioned using SOS1 variables $E B_{m, n}$. A bivariate model by Eqs. (1.B16)-(1.B18) is introduced to approximate the bilinear terms $B T_{p}$.

$$
\begin{aligned}
& T E I C \leq T E \cdot \varepsilon \\
& O B J_{\text {algorithm }}=\frac{i r \cdot\left(1-i r^{l s}\right)}{1-i r} \cdot A C F-T C I-r \cdot l s \cdot h \cdot T E
\end{aligned}
$$

To handle the fractional objective function, we introduce a parameter $r$ and an auxiliary problem with respect to $r$. The general model formulation of this auxiliary function is shown as follows.

$\max O B J_{\text {algorithm in }}(1 . \mathrm{B} 23)$

s.t. original constraints (1.A1)-(1.A45), (1.A47)-(1.A51), (1.A54)-(1.A57), (1.A59)-(1.A63), (1.A65)-(1.A79), (1.A81)-(1.A84) piecewise linear approximation functions (1.B1)-(1.B21) additional constraints for environmental objective functions (1.B22) new objective function (1.B23) 


\section{CHAPTER 5}

SUPERSTRUCTURE OPTIMIZATION COMBINED WITH PRODUCT

DISTRIBUTION OPTIMIZATION

\subsection{Introduction}

In this chapter, we propose a novel process synthesis framework that combines product distribution optimization of chemical reactions and superstructure optimization of the process flowsheet. In this framework, a superstructure that contains a variety of technology/process alternatives is developed in the first step. A set of dynamic optimization problems are solved to determine the optimal product distributions of the critical chemical reactions that maximize the profits of the effluent products. The optimal product distributions of these chemical reactions are then used as input parameters for extensive process simulations of the involved processes. Based on the high-fidelity process simulation results, a largescale mixed-integer nonlinear programming (MINLP) model is formulated to determine the optimal process design, which is further validated by a wholeprocess simulation in the last step of the proposed framework. We apply the proposed modeling framework to an integrated shale gas processing and chemical manufacturing process. The proposed superstructure includes 51,840 alternative possible process designs, and it consists of eight sections, including acid gas removal, dehydration, natural gas liquids (NGLs) recovery, NGLs separation, 
hydrocarbons conversion, light olefins separation, C4 separation, and acid gas disposal. For the steam cracking reactions in the hydrocarbons conversion section, we optimize the product distributions of steam cracking of ethane, propane, $n^{-}$ butane, and $i$-butane, because heavier components are usually sold as a natural gasoline product. Extensive process simulations are performed for all the involved processes in the superstructure in order to collect high-fidelity process data and develop detailed process models for the technology/process alternatives in the superstructure. Next, we propose a multiobjective MINLP model for sustainable design and synthesis of the integrated shale gas processing and chemical manufacturing process. The superstructure optimization model consists of five groups of constraints, namely superstructure network configuration constraints, mass balance constraints, energy balance constraints, techno-economic evaluation constraints, environmental impact assessment constraints. Three objective functions are maximizing the net present value (NPV) per GJ of raw shale gas, minimizing the global warming potential (GWP) per GJ of raw shale gas, and minimizing the water footprint per GJ of raw shale gas, respectively. Because the process system's performance and the total amount of raw shale gas processed are both considered in the unit objective functions, the optimal objective function values can directly reflect the competitiveness of the optimal process design. A 
tailored global optimization algorithm is applied to efficiently solve the resulting nonconvex MINLP problem. The application of the proposed framework is illustrated through a case study based on a Marcellus shale gas feed. The novelties of this work are summarized below:

- A novel modeling framework for process synthesis by combining product distribution optimization of chemical reactions and superstructure optimization of the process flowsheet;

- The first superstructure for an integrated shale gas processing and chemical manufacturing process systems including 51,840 alternative possible process designs;

- A novel product distribution optimization model for the steam cracking of hydrocarbons;

- A tailored global optimization algorithm for efficiently solving the resulting largescale nonconvex MINLP problem.

The rest of the paper is organized as follows. The problem addressed in this chapter is presented in the next section, followed by the Modeling Framework section. The proposed framework is applied to the process synthesis of an integrated shale gas processing and chemical manufacturing process. The superstructure of the integrated process system and corresponding optimization models are introduced next. We present the computational performance and the optimal solutions in the Results and Discussion section. Conclusion is provided at the end. 


\subsection{Problem statement}

The superstructure optimization problem for process synthesis of an integrated shale gas processing and chemical manufacturing process is formally stated in this section. We are given a set of technology/process alternatives in each section of the integrated shale gas processing and chemical manufacturing process. A superstructure can be developed as a collection of alternative possible process designs. The parameters that are relevant to the superstructure optimization problem include:

- The physical properties of the involved species;

- The ideal gas constant;

- The kinetic models of steam cracking of ethane, propane, $n$-butane, and $i$ butane [272, 273], including the Arrhenius parameters of each rate constant as well as the equilibrium constant of each reversible reaction;

- The heat of all reactions;

- The overall heat transfer coefficient between the wall of a steam cracking reactor and the gas mixture;

- The upper and lower bounds of the temperature, pressure, and feed-tosteam ratio of the gas mixture in each steam cracking reactor;

- The wall temperature and the diameter of the steam cracking reactors;

- The flow rate, the temperature, and the pressure of each hydrocarbon feed to its steam cracking reactor;

- The upper and lower bounds of the capacity of each technology/process;

- The composition and total flow rate of the raw shale gas;

- The composition of the feed to each technology/process; 
- The product distributions of the involved reactions;

- The split fraction of each species to various flows;

- The unit consumption and generation rates of power, heating, and cooling utilities in each technology/process;

- The steam turbine efficiency for electricity generation;

- The total hours of operations in a year;

- The project lifetime;

- The base-case capital costs, flow rates, scaling factors, and chemical engineering plant cost indices of the involved processes;

- The coefficients for evaluating total project investment based on capital costs;

- The prices of feedstocks, power, heating, and cooling utilities, and wastewater treatment;

- The coefficients for evaluating operating costs;

- The characterization factors of producing feedstocks, power, heating, and cooling utilities, wastewater treatment, and off-gas emissions.

The goal is to determine the optimal process design by maximizing the NPV per GJ of raw shale gas, minimizing the GWP per GJ of raw shale gas, and minimizing the water footprint per GJ of raw shale gas. The decision variables include:

- The flow rates of all the species along the steam cracking reactors;

- The temperatures of the gas mixture along the steam cracking reactors;

- The pressures of the gas mixture along the steam cracking reactors;

- The selection of technologies/processes in the optimal process design;

- The flow rates in each technology/process in the optimal process design;

- The NPV, capital expenditures (CAPEX), and operating expenditures (OPEX) of the optimal process design; 
- The GWP and the water footprint of the considered life cycle stages in the optimal process design.

\subsection{A novel modeling framework for process synthesis combining}

\section{reaction product distribution optimization and superstructure}

\section{optimization}

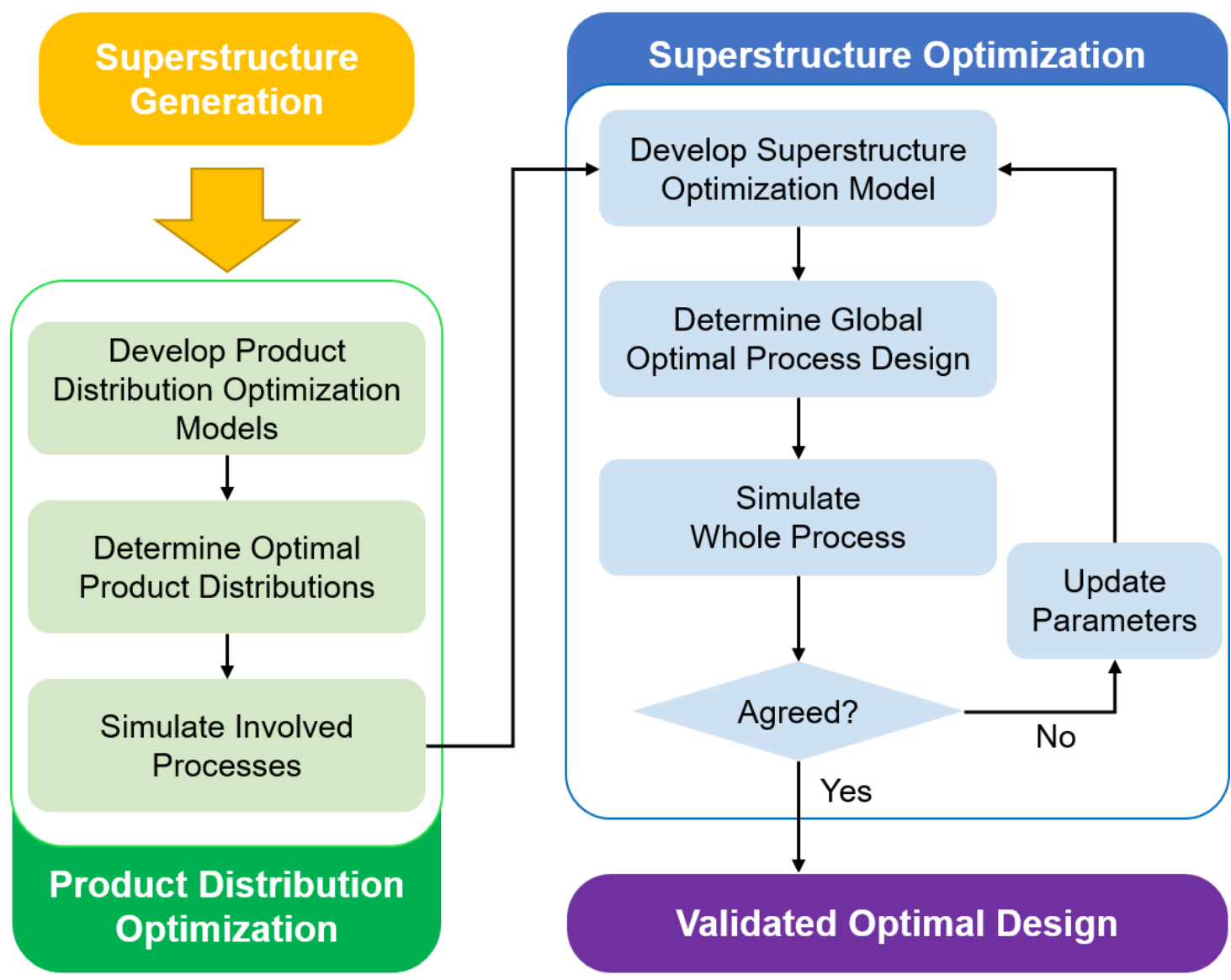

Figure 40. Flowchart of the proposed process synthesis framework that combines product distribution optimization of chemical reactions and superstructure optimization of the process flowsheet.

As product distributions of chemical reactions can significantly influence the overall performance of a process, it is important to account for product 
distributions of chemical reactions in a holistic framework for process synthesis. In this chapter, we propose a novel process synthesis framework that combines product distribution optimization of chemical reactions and superstructure optimization of the process flowsheet, as shown in Figure 40. In the first step, a superstructure is developed with a set of technology/process alternatives. In the second step, several product distribution optimization models for the involved chemical reactions are developed to maximize the profits of the effluent products. After the optimal product distributions of the chemical reactions are determined, they are used as input parameters for extensive process simulations to generate high-fidelity data for process synthesis. In the third step, the simulation results are used to develop a superstructure optimization model, which is likely a nonconvex MINLP. After globally optimizing the MINLP problem, the resulting optimal process design is then validated by a whole-process simulation. If the process flow rates of the optimal process design agree with those in the whole-process simulation, the framework would output the validated optimal design and terminate; otherwise, the process data in the whole-process simulation would be used to update the parameters in the superstructure optimization model, and the MINLP problem is solved iteratively until the process flow rates of the optimal process design agree with those in the whole-process simulation. As there are 
finite process designs in the superstructure and the parameters of one process design are updated in each iteration, the optimal process design converges to the corresponding whole-process simulation within finite iterations.

Ideally, the reaction product distribution optimization and superstructure optimization problems should be solved simultaneously in an integrated optimization framework. However, the explicit process models that treat product distributions of chemical reactions as variables are typically not available in the literature. More importantly, the resulting integrated problem is computationally intractable due to the computational complexities of the integrated optimization problems [274]. A superstructure optimization problem consists of integer variables to describe the network configuration and facilitate the process designs. Additionally, nonlinear terms are employed to evaluate capital costs and model other nonlinear relationships in the process. Due to the combinatorial nature and nonconvexity of the MINLP problem, it is nontrivial to globally optimize the superstructure optimization problem. To determine the optimal product distributions of the involved chemical reactions simultaneously, the integrated optimization problem should also embed a set of continuity equations and reaction kinetic models into the superstructure optimization problem. As a result, a set of ordinary differential equations (ODEs) and more nonlinear terms are added to the 
superstructure optimization problem, resulting in a large-scale mixed-integer nonlinear dynamic optimization problem, which is known to be very challenging to solve. Thus, the proposed framework employs a sequential heuristic approach that first determines the optimal product distributions of the involved chemical reactions, and then determines the optimal process design with the process data tailored to the optimal product distributions of the chemical reactions. For comparison purposes, the superstructure optimization problem is solved twice to determine the optimal solutions corresponding to the optimal product distributions and the product distributions taken from the literature, respectively. As illustrated by the shale gas application, the proposed approach is computationally tractable and leads to better solutions than the conventional superstructure optimization method that uses fixed product distributions of chemical reactions from literature. 


\subsection{Superstructure description}

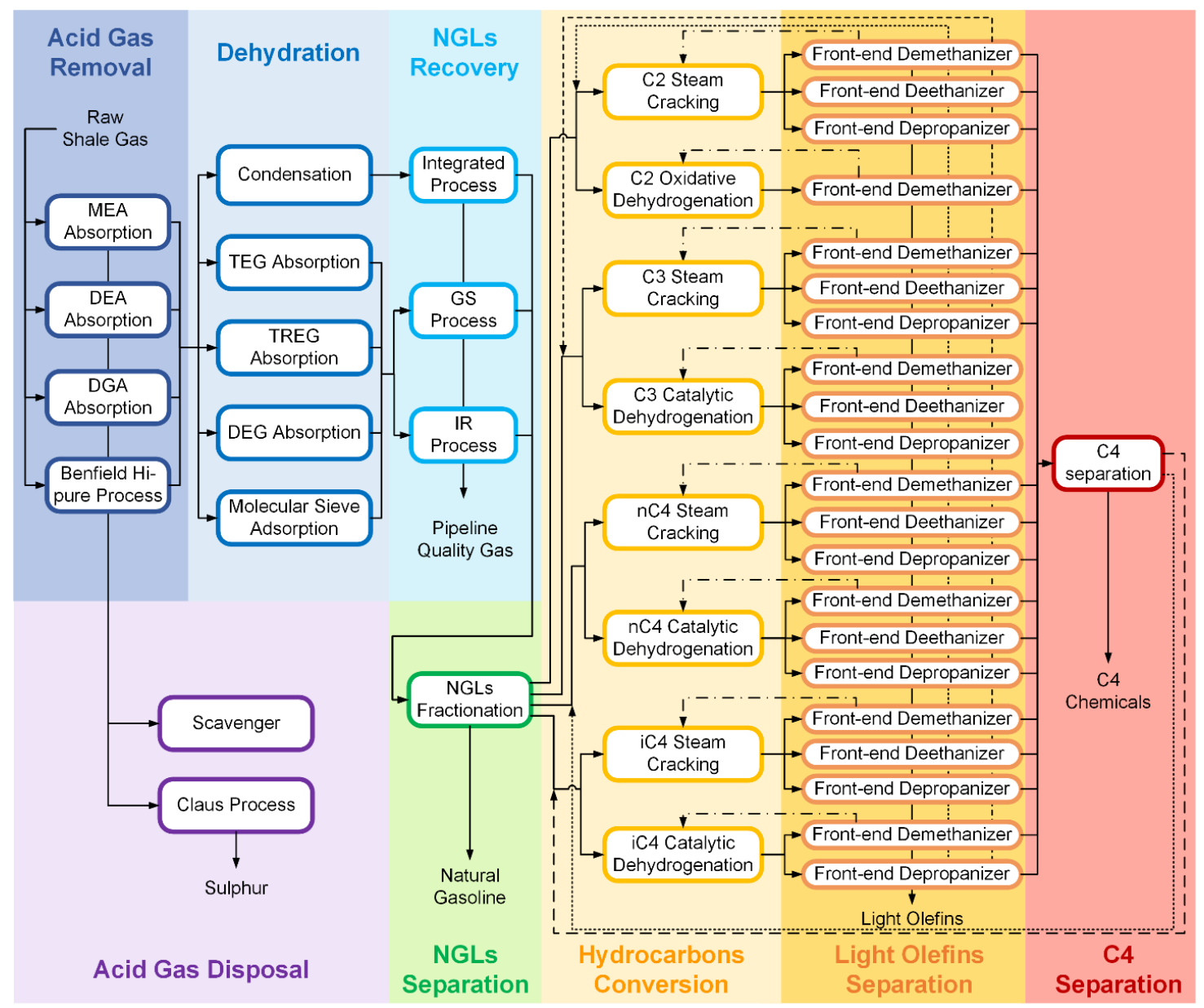

Figure 41. Overview of the proposed superstructure for an integrated shale gas processing and chemical manufacturing process.

The proposed modeling framework is applied to the process synthesis of an integrated shale gas processing and chemical manufacturing process. The details of the three key steps, i.e. superstructure generation, reaction product distribution optimization, and superstructure optimization, are introduced in this and the next two sections, respectively. 
Although several design variants for the polygeneration of chemicals and electricity from shale gas have been investigated [275-277], many other valuable technology and process alternatives may further improve the overall performance of an integrated system. In this chapter, we develop a comprehensive superstructure of an integrated shale gas processing and chemical manufacturing process to produce pipeline quality sales gas and a collection of value-added chemicals from raw shale gas. The general configuration of the proposed superstructure is shown in Figure 41 to provide an overview of the considered technology/process alternatives. There are eight sections in the superstructure, namely acid gas removal, dehydration, NGLs recovery, NGLs separation, hydrocarbons conversion, light olefins separation, C4 separation, and acid gas disposal. The raw shale gas from wellhead is first sent to the acid gas removal section to split hydrogen sulfide and carbon dioxide. The acid gas waste is then sent to the acid gas disposal section, and the sulfur content is captured before the remaining waste is emitted. The sweet gas becomes dry gas after the dehydration section. The dry gas is then separated by the NGLs recovery section to a pipeline gas product and a NGLs product. In the NGLs separation section, the mixture is split into ethane, propane, $n$-butane, $i$-butane, and natural gasoline. The first four hydrocarbons are sent to their corresponding processes in the hydrocarbons 
conversion section to form a spectrum of olefins. The effluents are then handled by a set of separation processes in the light olefins separation section. In additional to the light olefin products, the unreacted ethane and propane are recycled to the hydrocarbons conversion section, and the $\mathrm{C} 4$ mixture is sent to the $\mathrm{C} 4$ separation section. The unreacted $\mathrm{C} 4$ hydrocarbons are recycled to the hydrocarbons conversion section, while other C4 chemicals are fractionated to their corresponding products. The process flowsheet of the acid gas removal, acid gas disposal, dehydration, NGLs recovery, and NGLs separation sections is shown in Figure 42. The process flowsheet of the remaining sections is presented in Figure 43. Each section is described in the following subsections. 


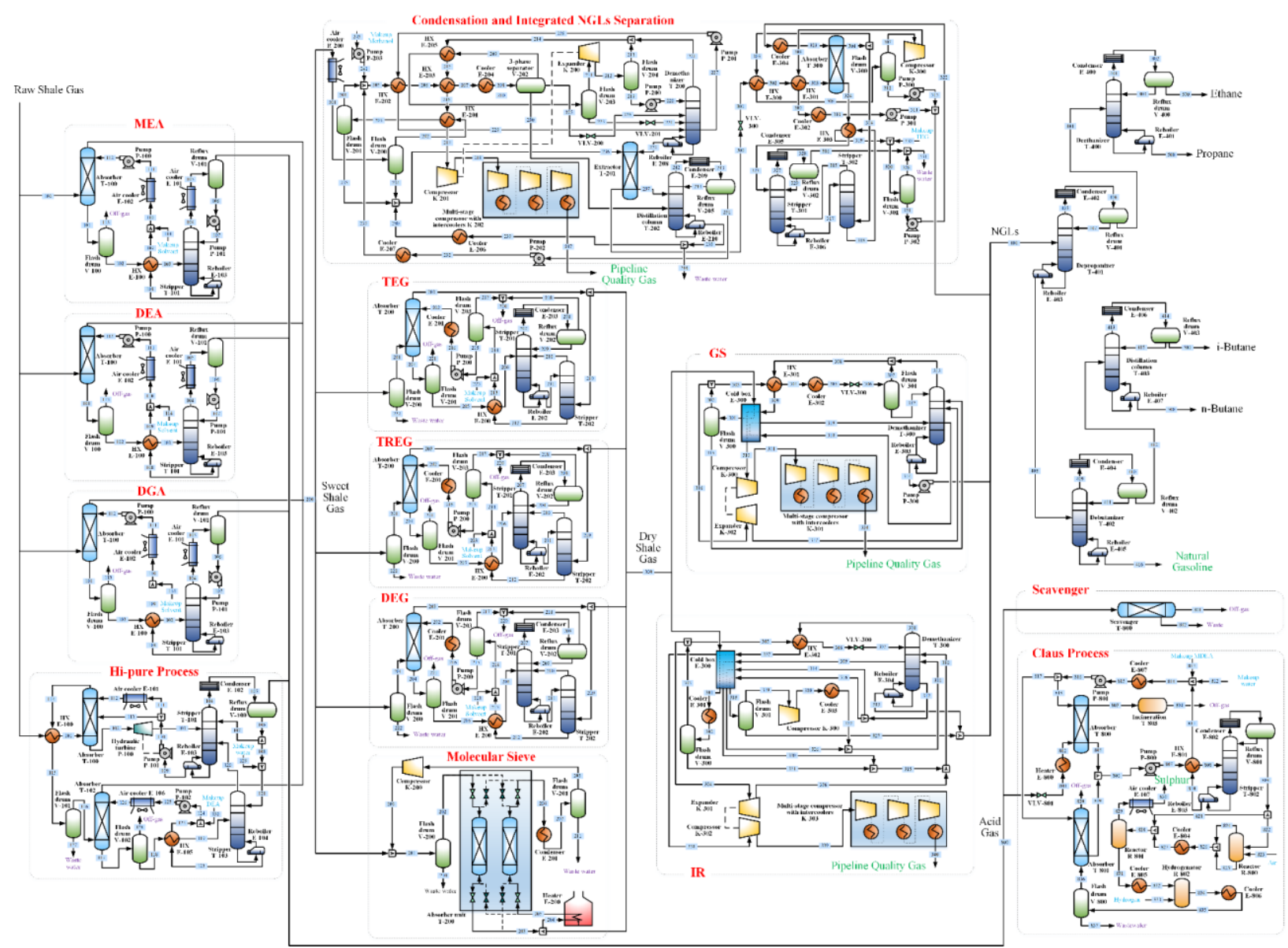

Figure 42. Process flowsheet of the acid gas removal, acid gas disposal, dehydration, NGLs recovery, and NGLs separation sections in the superstructure. 


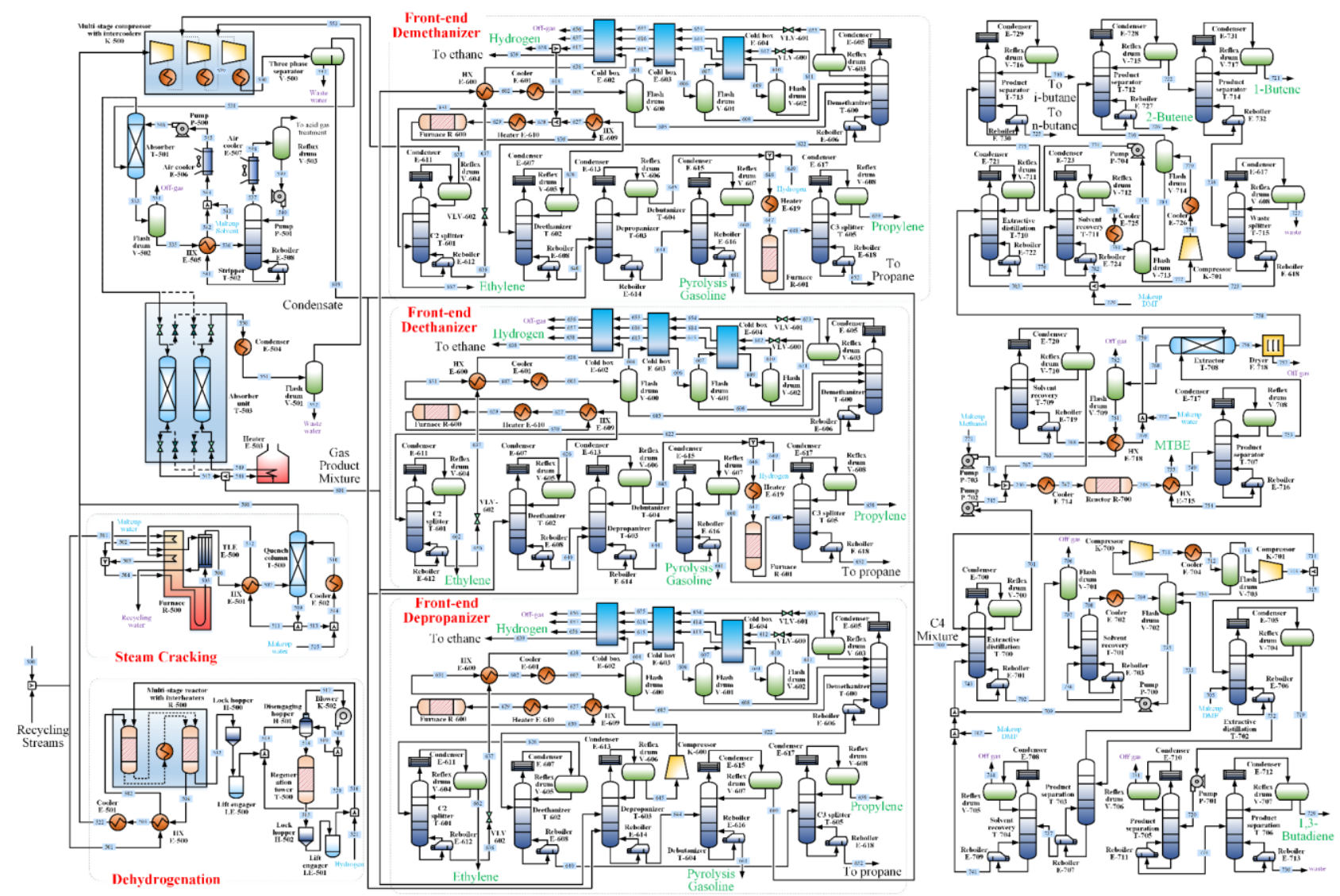

Figure 43. Process flowsheet of the hydrocarbons conversion, light olefins separation, and C4 separation sections in the superstructure. 


\subsubsection{Acid gas removal and acid gas disposal}

Raw shale gas is first introduced into the acid gas removal section to separate carbon dioxide and hydrogen sulfide from the hydrocarbons, so that no corrosive acid can be formed in the transmission pipelines [278]. Four technologies are considered to generate sweet shale gas in the superstructure, namely monoethanolamine (MEA) absorption, diethanolamine (DEA) absorption, diglycolamine (DGA) absorption, and Benfield Hipure process [278, 279]. The first three technologies follow the same process configuration of an absorption-stripper cycle while the selection of different solvents results in different operating conditions. MEA shows a higher solution capacity, but also a higher rate of vaporization loss and a higher energy requirement during regeneration [278]. In contrast, DGA has a lower vapor pressure and the corresponding process requires a lower solvent circulation rates. The DEA process, on the other hand, requires less energy for solvent regeneration. The Benfield Hipure process employs a hot potassium carbonate solution for absorbing most acid gas. Since conventional hot potassium carbonate based technologies are unable to reduce the concentration of $\mathrm{CO}_{2}$ to lower than $30 \mathrm{ppmv}$, an MEA absorption process is integrated into the Benfield Hipure process to further purify the resulting gas [280]. 
In the acid gas disposal section, the sulfur content in the acid gas is handled by either a scavenger or a Claus process. A scavenger, such as iron sponge, reacts with hydrogen sulfide to form non-regenerable wastes [278]. This option is costeffective if the amount of hydrogen sulfide is small; otherwise, a Claus process is more economically viable. Given that the sulfur concentration is too low to be thoroughly reacted with oxygen, a methyl diethanolamine absorption process is employed to concentrate hydrogen sulfide in the acid gas [26]. The sulfur content finally becomes solid sulfur and adds to the revenue of the entire project.

\subsubsection{Dehydration}

Water in the sweet shale gas can form hydrates in the following NGLs separation process. Therefore, five dehydration technologies are considered in the second section. The first one removes water when the sweet shale gas is condensed. The corresponding process shows a lower capital investment, but a higher utility cost than a triethylene glycol (TEG) absorption based process [116]. In addition to the condensation process, we include three glycol absorption based technologies. The diethylene glycol based process can operate under low temperatures; tetraethylene glycol based process shows a low circulation rate but a low hydrocarbon recovery [281]. Conventional TEG based process exhibits a comprised performance between the other two glycol based processes. The last alternative in the dehydration 
section is molecular sieve adsorption, which consumes more heating utility for a higher hydrocarbon recovery [280].

\subsubsection{NGLs recovery and NGLs separation}

The goal of the NGLs recovery section is to split pipeline quality gas from NGLs. We include three alternative processes. The first one is seamlessly integrated with the condensation process in the last section. In this process, methanol is added to the sweet shale gas as an inhibitor. To remove methanol in the NGLs product, a water wash tower and a TEG absorption process are employed. Note that the capacity of the added TEG absorption process is substantially smaller than that of the TEG absorption process in the dehydration section [116]. The other two alternative processes can handle the dry shale gas from the glycol based processes and the molecular sieve absorption process in the last section. A gas sub-cooled (GS) process employs a collection of heat exchangers, a Joule-Thompson valve, and a turboexpander for reaching a substantially low temperature before the feed gas is sent to a demethanizer column $[26,27,111]$. Based on the GS process, an internal refrigeration (IR) process cools the feed gas using a side draw from the bottom of the demethanizer column, aiming to reduce the cooling utility consumption [282]. For all these processes, pipeline quality gas is pressurized as a major product of the proposed superstructure. The other product stream containing NGLs is sent to the 
NGLs separation section, where the mixture is split into ethane, propane, $n^{-}$ butane, $i$-butane, and natural gasoline. Ethane, propane, $n$-butane, and $i$-butane are sent to the next section, and natural gasoline is sold as a product.

\subsubsection{Hydrocarbons conversion}

Given different reaction conditions, the four hydrocarbon feeds from the last section are sent to their respective reactors in the hydrocarbons conversion section. Two types of reactors are considered in the superstructure for each hydrocarbon feed: (1) steam cracking and oxidative dehydrogenation for ethane; and (2) steam cracking and catalytic dehydrogenation for propane, $n$-butane, and $i$ butane. For the steam cracking technology, the feed is sent to a furnace with steam and cracked into light alkanes and olefins in a few seconds [283]. To increase the

olefin yields, the reaction should be ceased instantaneously by rapidly cooling the furnace effluents through a transfer line exchanger and a water-quench column. After the cooling units, the gas mixture then reaches the ambient temperature. Later it is sent to a multi-stage compressor system, an MEA absorption process, and a molecular sieve adsorption unit for compression, acid gas removal, and drying, respectively. If the feed gas of a steam cracking process contains primarily heavier hydrocarbons, there can be an additional liquid stream formed during the compression process and it would be routed to the depropanizer column in the 
next section. The gas streams from the steam cracking processes are sent to corresponding separation processes in the following sections. There are many species in the effluents of steam cracking reactors, but the yields of olefins are relatively low. An attractive alternative to steam cracking of ethane is oxidative dehydrogenation of ethane, which offers a substantially higher ethylene yield and

consumes less energy [284]. In the same spirit, catalytic dehydrogenation of propane, $n$-butane, and $i$-butane enhances the yields of major olefin products and reduces the energy consumption [285]. Since the reactions are endothermic, heat consumption is supplied by inter-stage heaters. The supporting units for compression, acid gas removal, and dehydration are the same as those in the steam cracking processes. However, an additional catalyst regeneration unit is included in dehydrogenation processes [286].

\subsubsection{Light olefins separation}

Three alternative light olefins separation configurations, namely front-end demethanizer, front-end deethanizer, and front-end depropanizer, have gained commercial significance [283]. As their names suggest, the major difference lies in the first step in the fractionation sequence. In the front-end demethanizer configuration, the gas feed is first sent to a demethanizer column to remove methane and lighter components. The heavier stream is then sent to a deethanizer 
column, with the $\mathrm{C} 2$ components being hydrogenated and the $\mathrm{C} 3+$ components being depropanized. The treated C2 gas contains no acetylene, and it is split into ethylene and ethane products. The overhead gas from the depropanizer column is hydrogenated, and then split into a propylene product and a propane stream for recycling. The bottom gas from the depropanizer column is split into a pyrolysis gasoline product and a C4 stream to the next section. The separation sequence of the front-end deethanizer configuration starts from the deethanizer column. The lighter components are hydrogenated before being fed to a demethanizer column, and the heavier components are sent to a depropanizer column as in the front-end demethanizer configuration. In the front-end depropanizer configuration, the feed gas is sent to a depropanizer and the overhead product is pressurized before being sent to the hydrogenation reactor. Only one hydrogenation reactor is needed in the front-end depropanizer configuration as acetylene and methylacetylene are converted to respective olefins in the same reactor. Next, the treated gas is demethanized, deethanized, and split to an ethylene product, a propylene product, an ethane recycling stream, and a propane recycling stream. The bottom product from the depropanizer column is separated into a pyrolysis gasoline product and a C4 stream to the next section. When the feeds lack certain components, several unit operations or even the whole configuration become redundant. For the feeds 
with various percentages of olefins, the operating conditions can vary significantly. All three configurations are applied to each gas mixture product from the hydrocarbons conversion technologies with careful adjustment. A recycling stream containing methane and ethylene from the front-end demethanizer configuration is sent to the corresponding upstream hydrocarbons conversion process. The ethane streams from all light olefins separation processes are merged with the feed to the ethane conversion process, while all propane streams are merged with the feed to the propane conversion process.

\subsubsection{C4 separation}

The C4 mixture from the light olefins separation section consists of $n$-butane, $i$ butane, 1-butene, 2-butene, $i$-butene, and 1,3-butadiene. Extractive distillation with dimethylformamide (DMF) is first employed to separate 1,3-butadiene [287, 288]. Next, methanol is added to the remaining gas mixture to turn $i$-butene to methyl tert-butyl ether (MTBE) [289]. MTBE is separated as the bottom product of a distillation column, and the residual methanol is washed off and recycled. The dried gas is extracted by DMF again with butanes remaining in the gas phase. $n^{-}$ Butane and $i$-butane are then split and recycle. Finally, the extracted components from the top of a solvent recovery column are fractionated to $n$-butene products sequentially [290]. 


\subsection{Product distribution optimization of chemical reactions}

In the second step of the proposed framework, product distribution optimization models are developed for the critical reactions in the superstructure. In the integrated shale gas processing and chemical manufacturing process, steam cracking is a well-established reaction for producing olefins in the petrochemical industry [283]. With the kinetic models of steam cracking [272, 273], it is possible to determine the optimal product distributions of chemical reactions that maximize the profit of the effluent products under certain market conditions. Compared with the product distributions of chemical reactions in the literature that might not be optimized based on the current market conditions, the optimization can enhance the yields of more profitable products, thus contributing to a better overall performance of the entire system. It is practical to integrate chemical kinetic models into rigorous simulation models for process design [276, 277], but how to determine the optimal product distributions of chemical reactions systematically in superstructure optimization is still a research challenge. Ideally, an integrated optimization model should be developed for simultaneous reaction product distribution optimization and superstructure optimization. However, it is challenging to develop the explicit process models that treat product distributions of chemical reactions as variables. Moreover, the integration of these optimization 
models combines their computational complexities. With a set of integer variables as well as nonconvex and nonlinear ODEs, it is challenging to globally optimize the resulting large-scale integrated optimization problem. As a heuristic approach, we solve the reaction product distribution optimization problems and the superstructure optimization problem sequentially in this chapter. The optimal product distributions of the involved chemical reactions that maximize the profits of the effluent products are used as input parameters of rigorous process simulations. The high-fidelity process simulation data can be used in the superstructure optimization problem to determine the optimal process design. Additionally, we apply a tailored global optimization algorithm to efficiently solve the resulting large-scale nonconvex MINLP problem for superstructure optimization.

In this section, we present the product distribution optimization models for steam cracking. The hydrocarbons that are heavier than butane in the NGL stream are typically separated and sold to the market as a natural gasoline product [283]. Therefore, we focus on steam cracking of ethane, propane, $n$-butane, and $i$-butane in this study. We develop four ODE-constrained dynamic optimization models for steam cracking of these hydrocarbons, respectively. The general model formulation 
is shown below. The detailed models and optimization results are presented in Appendix A.

$\max \quad P R O$ as defined in (1.A48)

s.t. $\quad$ kinetic model constraints (1.A24)-(1.A30) continuity equations and boundary conditions (1.A31)-(1.A43) economic evaluation constraints (1.A44)-(1.A48)

\subsection{Superstructure optimization for sustainable process design and synthesis}

\subsubsection{Model formulations for life cycle optimization}

Based on the optimal product distributions of steam cracking of ethane, propane, $n$-butane, and $i$-butane, we develop 53 simulation models for different alternative designs of processes in the superstructure. In the light olefins separation section, 12 simulation models are developed for the steam cracking processes with the optimal product distributions, 12 simulation models are developed for the steam cracking processes with the product distributions taken from the literature [283, 285], and another 9 simulation models are developed for the dehydrogenation processes. The simulation results are then used to calculate key parameters such as inlet compositions and split fractions of the major unit operations in the process. Finally, a life cycle optimization model is formulated to determine the optimal process design of the integrated shale gas processing and chemical manufacturing process 
$[12,22,197]$. The life cycle optimization model integrates superstructure optimization and the tenets of the techno-economic analysis and life cycle analysis (LCA) methodologies through a multiobjective optimization framework [9].

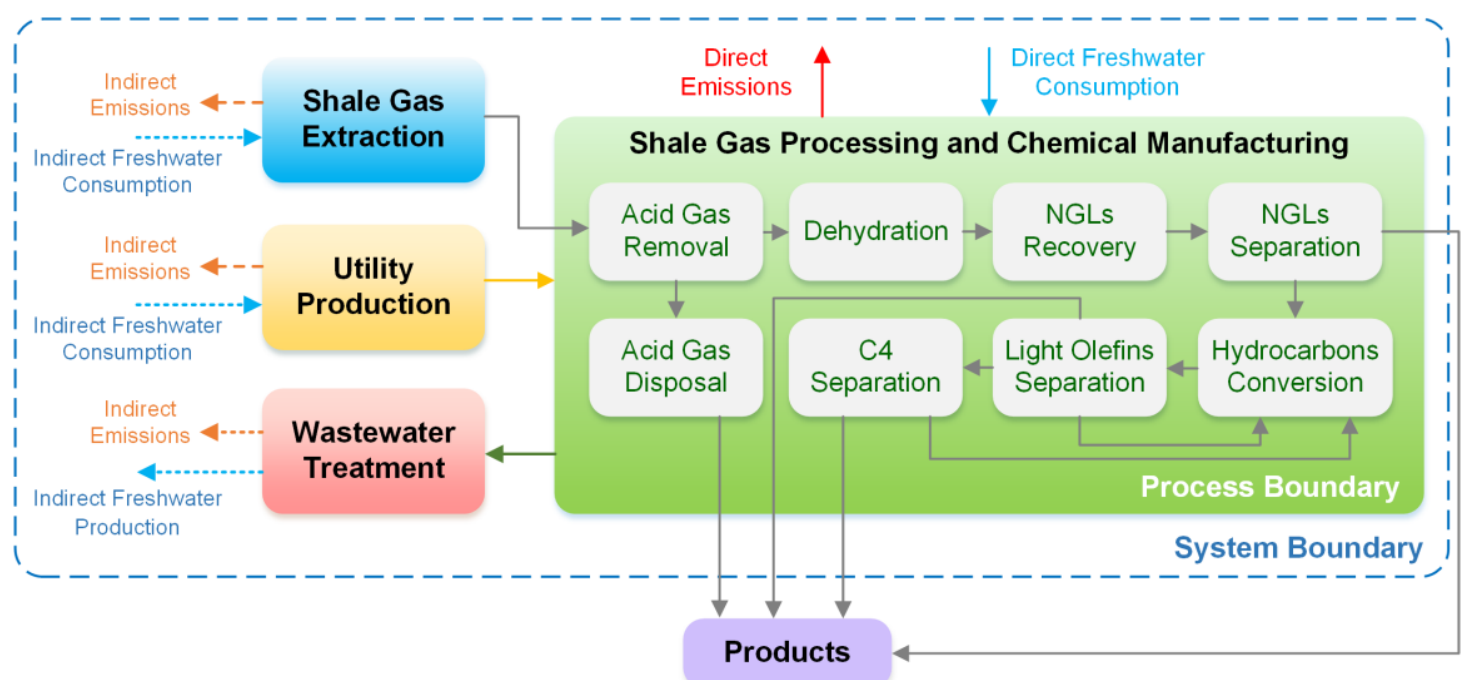

Figure 44. System boundary of the cradle-to-gate life cycle optimization. The gray arrows denote the material flows; the yellow arrow denotes the utility flow; the dark green arrow, the red arrow, and the solid blue arrow denote the wastewater, direct emissions, and direct water consumption from the integrated shale gas processing and chemical manufacturing process, respectively; the dotted orange arrows and the dotted blue arrows represent indirect emissions and indirect water consumption and production, respectively.

The functional unit of the LCA is processing $1 \mathrm{GJ}$ raw shale gas. As shown in Figure 44, the system boundary covers four life cycle stages from cradle to gate, namely shale gas extraction, utility production, shale gas processing and chemical manufacturing, and wastewater treatment. We focus on GWP and water footprint in life cycle impact assessment for two reasons. First, these two indicators are most widely discussed environmental impact indicators for shale gas systems [291-294]. Second, there are not enough life cycle impact assessment results of other mid- 
point or end-point environmental impact categories for the studied life cycle stages. It deserves future research effort to develop comprehensive life cycle inventories and life cycle impact assessment results for the integrated shale gas processing and chemical manufacturing process. GWP measures the global warming impact of a chemical relative to that of carbon dioxide, whose GWP is equal to 1 [265]. For example, emitting $1 \mathrm{~kg}$ of methane is equivalent to emitting $28 \mathrm{~kg}$ of $\mathrm{CO}_{2}$ over the course of 100 years according to the Intergovernmental Panel on Climate Change [265]. As shown in Figure 44, the GWP in this LCA accounts for the global warming impact of the greenhouse gases emitted in all the life cycle stages. Moreover, the water footprint in this LCA accounts for direct water consumption in the integrated shale gas processing and chemical manufacturing process, indirect water consumption in shale gas extraction and utility production, and indirect water production in wastewater treatment. The data for direct water consumption in the superstructure are directly extracted from the simulation results. The data for indirect water consumption associated with shale gas extraction and utility production are taken from the literature [293]. The data for indirect water production in the wastewater treatment process are taken from the Ecoinvent database [295]. 
The techno-economic analysis accounts for the CAPEX and the OPEX of the integrated shale gas processing and chemical manufacturing process. CAPEX includes the capital costs of reactors, columns, compressors, drums, and heat exchangers as well as land cost and working capital [46]. OPEX includes feedstock cost, utility cost, operations \& maintenance cost, property tax \& insurance, general expense, and income tax. The annual cash flow is equal to the difference between the annual revenue and the annual OPEX. The NPV is defined by subtracting the CAPEX from the sum of the discounted annual cash flows over a project lifetime of 20 years at an interest rate of $10 \%$. Zero salvage value is considered when calculating the NPV.

The general model formulation of the life cycle optimization model is given below, and the detailed models are presented in Appendix C. The proposed model employs functional unit based objective functions for several reasons. First, the functional unit in LCA provides a reference to which the inputs and outputs of a system can be related. By applying the same functional unit, the performance of different process systems that generate distinct final products at various scales can be compared in a fair manner. Second, the optimal performance of the entire process system may correspond to significantly low productivities. Thus, the resulting optimal process design may be less sustainable and the final products may 
be less competitive in the markets. In contrast, the functional unit based objective functions account for the entire process system's performance and the quantity of the final products simultaneously. The corresponding optimal solutions are found to be more sustainable and more competitive $[197,296]$.

$$
\begin{aligned}
& \max \quad O B J_{\text {economic }}=\frac{\sum_{i, j}\left[X_{i, j}+a_{i, j}\left(Z_{i, j}\right)^{n_{i, j}}\right]}{l t \cdot h \cdot T E C} \text { in Eq. (1.C36) } \\
& \min O B J_{G W P}=\frac{(E I F+E I U+E I W+E I D)_{G W P}}{T E C} \text { in Eq. (1.C41) } \\
& \min O B J_{\text {water }}=\frac{(E I F+E I U+E I W)_{\text {water }}}{T E C} \text { in Eq. (1.C42) }
\end{aligned}
$$

s.t. $\quad$ superstructure network configuration constraints (1.C43)-(1.C56) mass balance constraints (1.C1)-(1.C16) energy balance constraints (1.C17)-(1.C20) techno-economic evaluation constraints (1.C21)-(1.C36) environmental impact assessment constraints (1.C37)-(1.C42)

where $O B J_{\text {economic, }} O B J_{G W P}$, and $O B J_{\text {water }}$ denote the unit NPV, unit GWP and unit water footprint, respectively. The numerator of $O B J_{\text {economic }}$ is the NPV, which sums up several linear terms for the revenue and the OPEX (denoted by $X_{i, j}$ ) and several separable concave terms for the CAPEX (denoted by $a_{i, j}\left(Z_{i, j}\right)^{n_{i, j}}$ ). The numerator of $O B J_{\text {economic }}$ accounts for the total energy content generated over the project lifetime. $l t, h$, and $T E C$ are the project lifetime, the operating time per year, and the total energy content per hour, respectively. The numerators of $O B J_{G W P}$ and 
$O B J_{\text {water }}$ are the total GWP per hour and the total water footprint per hour, respectively. EIF, EIU, EIW, and EID represent the environmental impacts associated with feedstocks, utilities, wastewater treatment, and direct emissions, respectively. The constraints for mass and energy balance relationships are developed for the technology/process alternatives, instead of the equipment units In the life cycle optimization model, integer variables are introduced to select the technology/process alternatives and facilitate the process designs. The other decision variables, such as process flow rates, are continuous variables. Additionally, separable concave terms are used to calculate the CAPEX in the techno-economic evaluation constraints. The objective functions include mixedinteger fractional terms. Given the problem structure, the resulting problem is a mixed-integer nonlinear fractional programming (MINFP) problem and the global optimization of this problem is nontrivial.

\subsubsection{Tailored global optimization strategy}

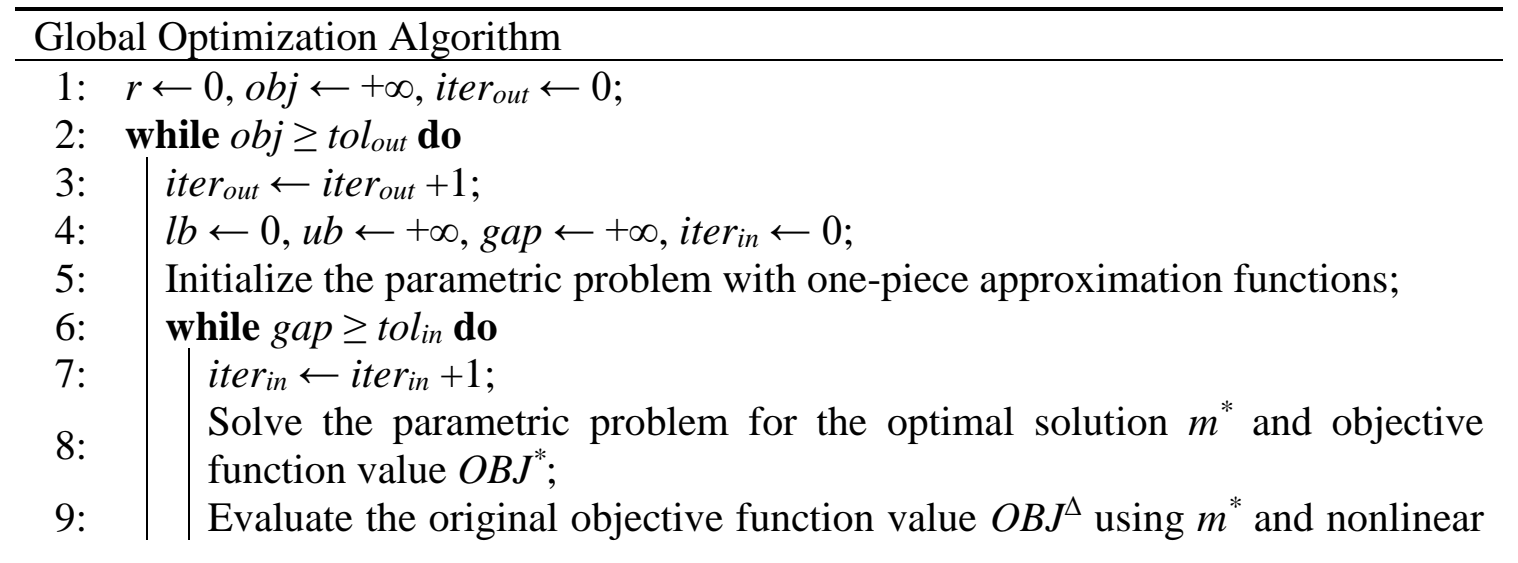




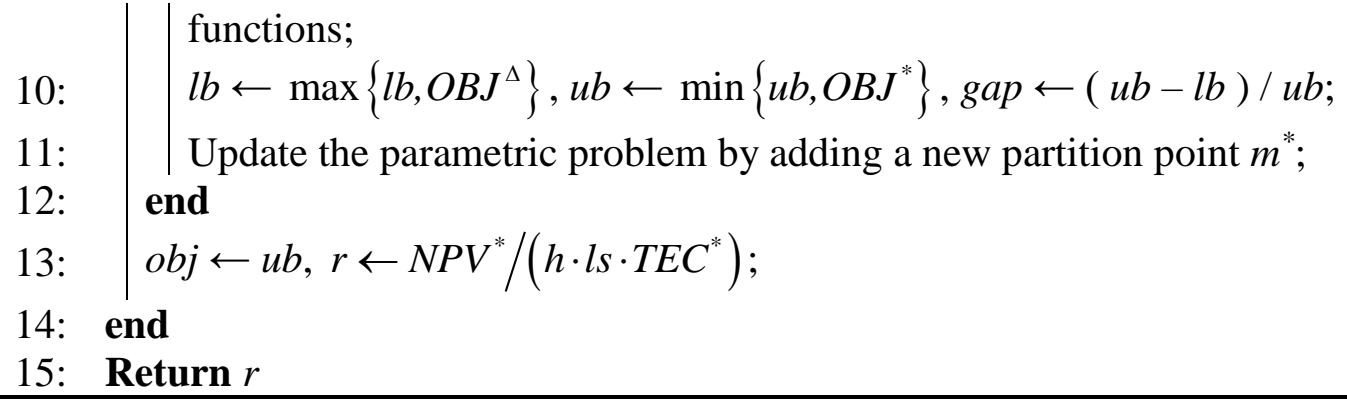

Figure 45 . The pseudo code of the tailored global optimization algorithm that integrates the inexact parametric algorithm and the branch-and-refine algorithm.

As mentioned above, the multiobjective MINFP problem consists of both integer and continuous variables as well as multiple nonlinear nonconvex functions. Due to the combinatorial nature and nonconvexity, global optimization of this MINFP problem can be computationally intractable for general-purpose global solvers [21]. To tackle the computational challenge, we employ a tailored global optimization algorithm to efficiently solve this MINFP problem [21]. Specifically, the global optimization algorithm integrates two state-of-the-art algorithms, including the inexact parametric algorithm [202] and the branch-and-refine algorithm [200].

The computational challenge stemming from the fractional objective functions is tackled by the inexact parametric algorithm. Instead of solving the original MINFP problem directly, we introduce an auxiliary parameter $r$ and an auxiliary parametric problem $F(r)$. The original optimal solution is identical to the optimal solution of the auxiliary parametric problem with the parameter $r^{*}$ such that $F\left(r^{*}\right)=0$ [202]. In each iteration of the inexact parametric algorithm, we need to 
globally optimize an MINLP problem $F(r)$ with separable concave terms in the objective function. To efficiently solve these MINLP problems, we replace the nonlinear terms with successive piecewise linear approximation functions and solve the relaxed mixed-integer linear programming (MILP) problems iteratively following the branch-and-refine algorithm [200]. This tailored solution algorithm is guaranteed to converge within finite iterations. The pseudo code of the tailored global optimization algorithm is shown in Figure 26. The iteration counters in the outer loop and the inner loop are denoted as iterout and iterin, respectively. The optimality tolerance in the outer loop and the inner loop are denoted as tolout and tolin, respectively. obj represents the objective function value in the outer loop, while $u b$ and $l b$ represent the upper bound and the lower bound in the inner loop, respectively. gap denotes the optimality gap in the inner loop. The global optimal solution in the inner loop is $m$. The optimal objective function value and the evaluated objective function value are $O B J$ and $O B{ }^{\AA}$, respectively. The detailed model formulation of the auxiliary parametric problem is given in Appendix D.

\subsection{Application to a Marcellus shale gas feed}

The proposed framework is applied to the design and synthesis of an integrated shale gas processing and chemical manufacturing process for handling a raw shale gas feed of 200 million standard cubic feet per day from Marcellus shale [297]. The 
raw shale gas feed is collected from about 200 wells after being preprocessed on wellsites [298]. All computational experiments are performed on a DELL OPTIPLEX 7040 desktop with Intel(R) Core(TM) i7-6700 CPU @ 3.40GHz and 32 GB RAM. The process simulation models are developed in ASPEN HYSYS [299]. We use the amine thermodynamic package in ASPEN HYSYS for the processes that involve amines [300], the glycol thermodynamic package for the processes that involve glycols [300], the Wilson thermodynamic package for the C4 separation process [301], and the Peng-Robinson thermodynamic package for the remaining processes [300]. The capital costs are evaluated using ASPEN Process Economic Analyser [302]. The product distribution optimization problems for steam cracking are coded and solved in MATLAB 2016a [303]. Specifically, the fmincon solver is employed to solve the product distribution optimization problems for steam cracking reactions, and the ode15s solver is used to handle the ODEs [304]. The superstructure optimization problem and its solution procedure are coded in GAMS 24.8.3 [203], with CPLEX 12.7. used as the MILP solver and BARON 17.1 [204] and SCIP 3.2 [305] being the MINLP solvers. The relative optimality tolerances for the inexact parametric algorithm and the branch-andrefine algorithm are $10^{-6}$. 


\subsubsection{Case study 1: Minimizing the unit GWP of shale gas processing}

A shale gas processing system receives raw shale gas and separates undesired components to produce pipeline quality gas. For gas quality and transmission safety purposes, shale gas processing is usually required regardless of the amount of NGLs in a raw shale gas feed. Therefore, many LCA studies for shale gas production and utilization calculate the environmental impacts associated with shale gas processing. In the first case study, we focus on the shale gas processing part of the superstructure, which consists of the acid gas removal section, the dehydration section, and the NGLs recovery section. As no hydrogen sulfide is reported in the considered raw shale gas feed, the acid gas disposal section is bypassed. Moreover, the sections involving chemical reactions are not considered in this case study, so product distribution optimiztion for chemical reactions is bypassed. To compare with existing LCA results, we focus on minimizing the GWP per GJ of the products from shale gas processing.

As shown in Table 9, the minimum GWP per GJ of the products from shale gas processing is $1.12 \mathrm{~kg} \mathrm{CO}-\mathrm{eq} / \mathrm{GJ}$. It is reported that the GWP per GJ of the products from shale gas processing from raw Marcellus shale gas is $1.20 \mathrm{~kg} \mathrm{CO}_{2}-\mathrm{eq} / \mathrm{GJ}$ [293], while the corresponding value for average raw shale gas in the U.S. is $3.66 \mathrm{~kg} \mathrm{CO}_{2-}$ eq/GJ [306]. A comparison shows that the minimum unit GWP of shale gas 
processing in the proposed superstructure is lower than the literature values. It is noted that different works may apply different assumptions and rely on data from various sources. Therefore, the goal of comparing the unit GWP values is to validate that the superstructure optimization model can return reasonable estimates of environmental impacts relative to those in the literature.

Table 9. Comparison of the GWP per GJ of the products from shale gas processing in this chapter and in the literature.

\begin{tabular}{ccc}
\hline Source & Unit GWP $\left(\mathrm{kg} \mathrm{CO}_{2}-\mathrm{eq} / \mathrm{GJ}\right)$ & Raw shale gas source \\
\hline This work & 1.12 & Marcellus \\
Laurenzi and Jersey & 1.20 & Marcellus \\
[293] & 4.30 & Marcellus \\
Jiang et al. [294] & 3.66 & National average \\
Burnham et al. [306] &
\end{tabular}

The selection of the optimal technology/process alternatives is the major reason for resulting in a lower unit GWP in this chapter than in the literature. In the cited literature [293], a TEG absorption process and a GS process are employed in the dehydration section and the NGLs recovery section, respectively. Although the TEG abosrption process does not require a dedicated heater or consume a large amount of fuel gas for heating, it demonstrates a lower hydrocarbon recovery rate, resulting in a higher unit GWP. The GS process, however, is less energy-efficient than the other process alternatives. In contrast, the most environmentally friendly shale gas processing design in this chapter selects the MEA absorption process, the 
molecular sieve adsorption process, and the IR process as shown in Figure 46. The molecular sieve adsorption process has a higher hydrocarbon recovery, resulting in more hydrocarbon products than the other alternatives. The IR process is energyefficient and shows a lower GWP associated with utility generation. Overall, the selection of a different set of technologies/processes leads to the minimum unit GWP.
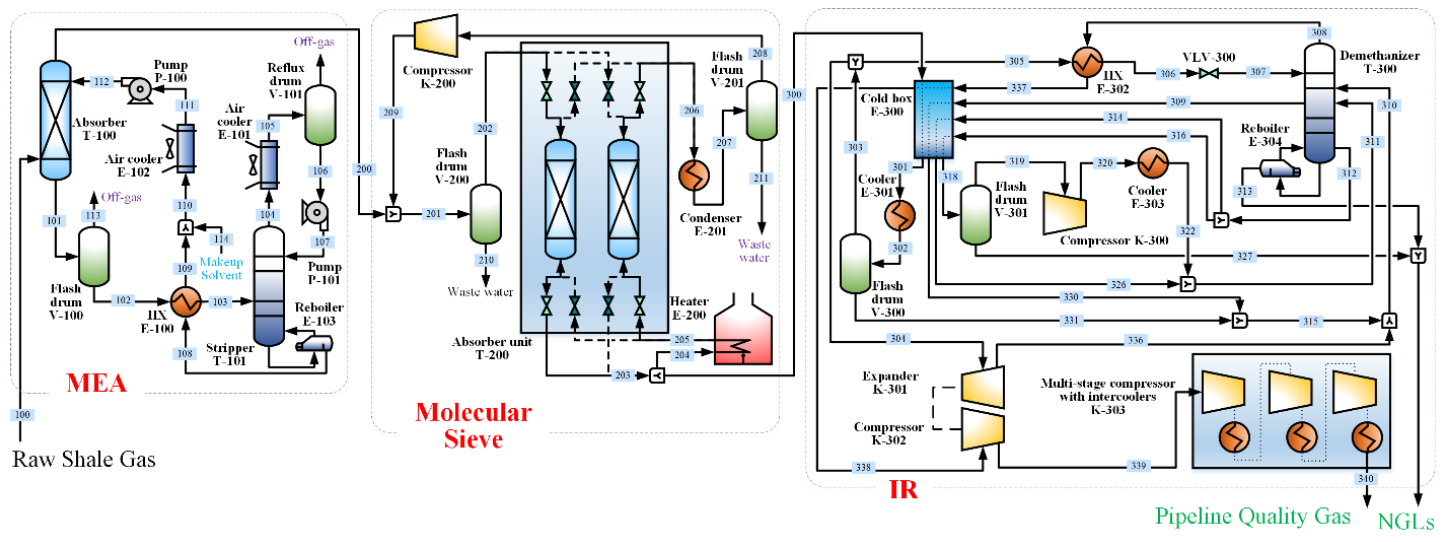

Figure 46. Optimal process flowsheet corresponding to the minimum GWP per GJ of the products from the shale gas processing part of the integrated shale gas processing and chemical manufacturing process.

\subsubsection{Case study 2: Economic and environmental optimization of the entire superstructure}

In the second case study, we consider the entire superstructure for the integrated shale gas processing and chemical manufacturing process. To better understand the trade-offs between the economic and environmental objectives, the first one maximizes the NPV per GJ of raw shale gas and minimizes the GWP per GJ of raw 
shale gas simultaneously; the second one maximizes the NPV per GJ of raw shale gas and minimizes the water footprint per GJ of raw shale gas simultaneously. The optimal solutions of these multiobjective optimization problems can be plotted as Pareto-optimal curves. The Pareto-optimal curves in Figure 47 demonstrate the trade-offs between the unit NPV and the unit GWP, while those in Figure 48 demonstrate the trade-offs between the unit NPV and the unit water footprint.

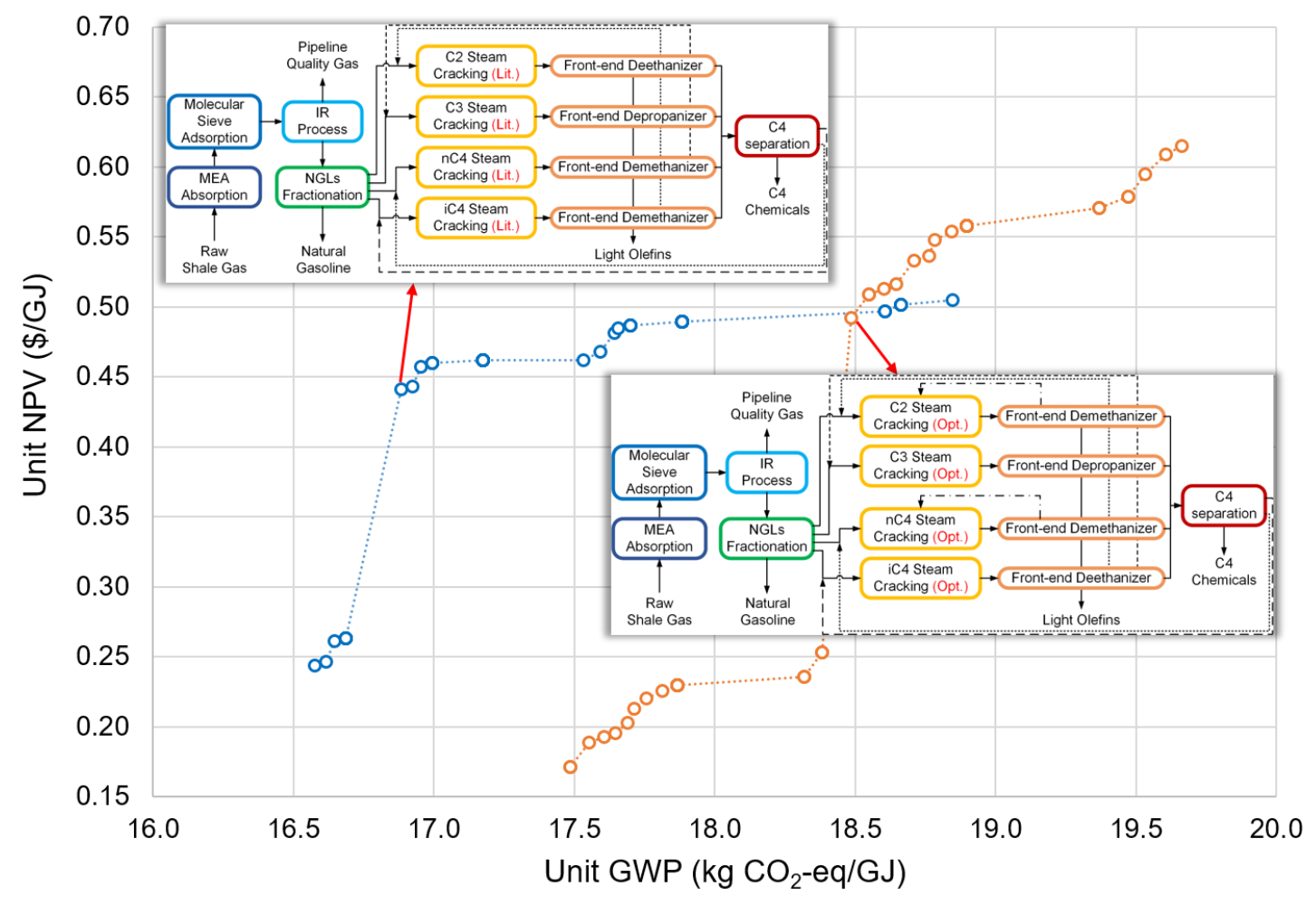

...... Product distributions taken from the literature $\cdots . . .$. Optimal product distributions

Figure 47. Pareto-optimal curves showing the trade-offs between the NPV per GJ of raw shale gas and the GWP per GJ of raw shale gas. Two good-choice optimal solutions and the selected technologies/processes are also presented. 
Each point on the Pareto-optimal curve corresponds to an optimal solution of the superstructure optimization problem. The highest unit NPV with the optimal product distributions of steam cracking is $\$ 0.62 / G$ J (corresponding NPV: $\$ 1.12$ billion), which is $22 \%$ higher than the highest unit NPV with the product distributions of steam cracking taken from the literature $[283,285]$. This improvement comes from the product distribution optimization of steam cracking, which considerably increases the yield of the profitable 1,3-butadiene. With the optimal product distributions of steam cracking, the economic performance of the optimal process designs can be enhanced. Moreover, the minimum unit GWP and minimum unit water footprint with the product distributions of steam cracking taken from the literature are $16.58 \mathrm{~kg} \mathrm{CO}_{2}-\mathrm{eq} / \mathrm{GJ}$ and $33.11 \mathrm{~kg} \mathrm{H}_{2} \mathrm{O} / \mathrm{GJ}$, respectively. In contrast, the minimum environmental impact indicators with the optimal product distributions of steam cracking are $17.49 \mathrm{~kg} \mathrm{CO}$-eq/GJ and 34.57 $\mathrm{kg} \mathrm{H}_{2} \mathrm{O} / \mathrm{GJ}$, respectively. To increase the yields of more profitable products, the temperatures and pressures of the steam cracking reactions need to be raised. As a result, the optimal process designs with the optimal product distributions consume more utilities, contributing to relatively higher unit environmental impacts. 


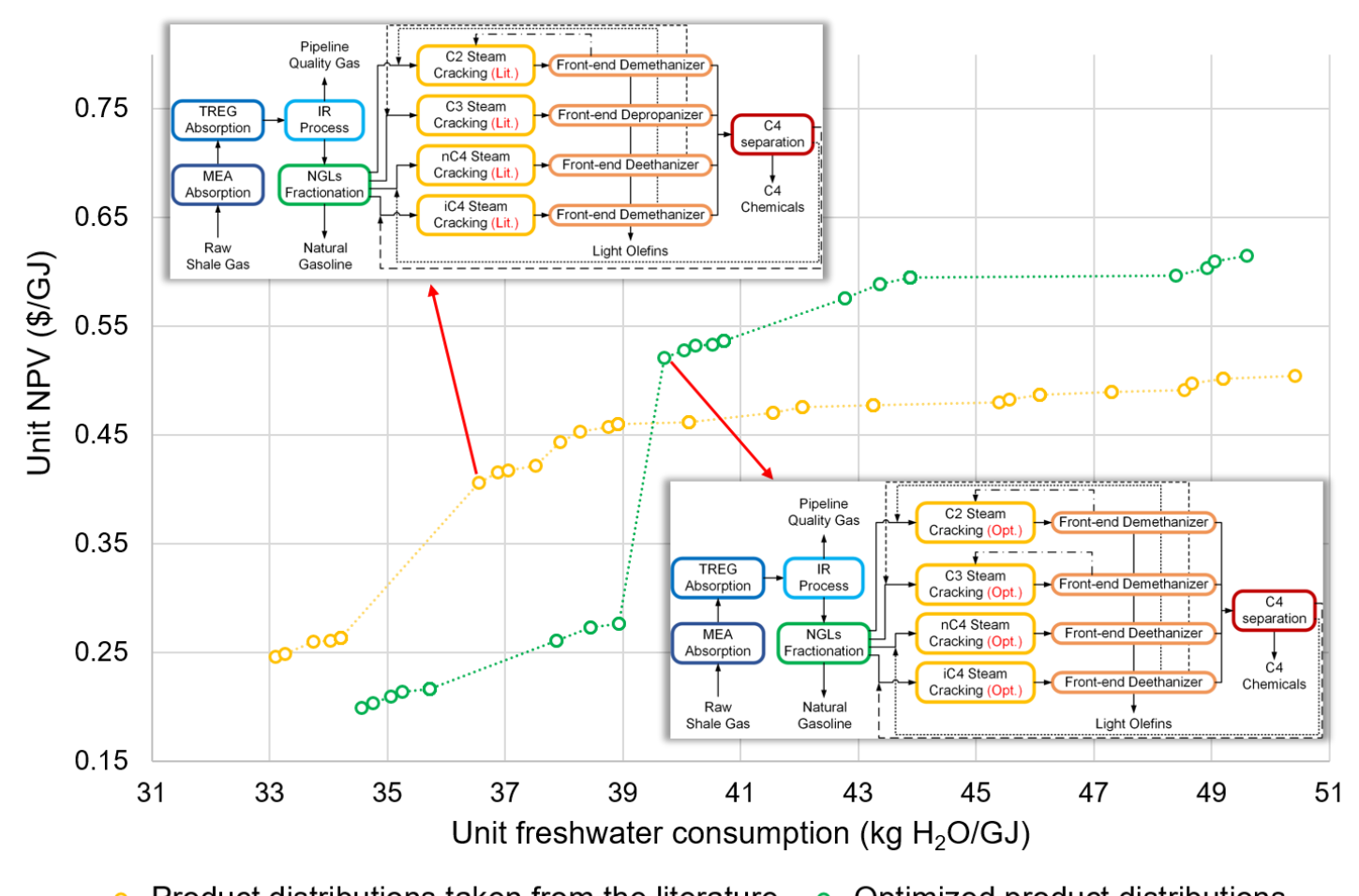

Figure 48. Pareto-optimal curves showing the trade-offs between the NPV per GJ of raw shale gas and the water footprint per GJ of raw shale gas. Two good-choice optimal solutions and the selected technologies/processes are also presented.

Each point on the Pareto-optimal curves corresponds to an optimal process design.

In addition to the Pareto-optimal curves, Figure 47 and Figure 48 exhibit the selected technologies/processes of four good-choice optimal solutions. The goodchoice optimal solutions in Figure 47 select a molecular sieve absorption process in the dehydration section, while those in Figure 48 select a TREG absorption process. In the glycol based dehydration processes, a portion of the dry shale gas product must be separated and utilized as stripping gas to reduce the water content in the recycled glycol solvent. Later, the stripping gas coming from the top of the 
stripper column is burnt in a furnace to generate heating utilities. Therefore, the gas yields of the glycol based dehydration processes are lower than that of the molecular sieve absorption process, which sends all the dry shale gas product to the next section. Despite this drawback, the TREG absorption process corresponds to much less water footprint than the molecular sieve absorption process, because the latter process relies on a significant amount of water to cool the gas product. Therefore, the optimal solution with the minimum GWP per GJ of raw shale gas favors molecular sieve absorption, while the optimal solution with the minimum water footprint per GJ of raw shale gas favors TREG absorption. Moreover, since steam cracking strikes a good balance between the economic and environmental performance, the good-choice optimal solutions select only steam cracking technologies in the hydrocarbons conversion section.

\subsubsection{Case study 3: Simultaneously maximizing the unit NPV, minimizing the unit GWP, and minimizing the unit water footprint of the entire superstructure}

In the third case study, we consider the entire superstructure and solve the superstructure optimization problem that maximizes the NPV per GJ of raw shale gas, minimizes the GWP per GJ of raw shale gas, and minimizes the water footprint per GJ of raw shale gas simultaneously. The optimal solutions of this 
multiobjective optimization problem can be plotted as 3D Pareto-optimal surfaces. Figure 49 shows the Pareto-optimal surface with the product distributions of steam cracking taken from the literature and Figure 50 shows the Pareto-optimal surface with the optimal product distributions of steam cracking.

\subsubsection{Optimal process designs}

The selected technologies/processes of a good-choice optimal solution are highlighted on each Pareto-optimal surface (A in Figure 49 and B in Figure 50). The optimal process flowsheets of the good-choice optimal solutions are explicitly demonstrated in Figure 51 and Figure 52. The unit NPV, the unit GWP, and the unit water footprint of the optimal solution A are $\$ 0.46 / \mathrm{GJ}$ (corresponding NPV: \$0.83 billion), $16.99 \mathrm{~kg} \mathrm{CO} 2-\mathrm{eq} / \mathrm{GJ}$, and $38.92 \mathrm{~kg} \mathrm{H} \mathrm{O} / \mathrm{GJ}$, respectively. In contrast, the unit NPV, the unit GWP, and the unit water footprint of the optimal solution B are $\$ 0.53 / \mathrm{GJ}$ (corresponding NPV: $\$ 0.96$ billion), $18.71 \mathrm{~kg} \mathrm{CO}$-eq/GJ, and 40.42 $\mathrm{kg} \mathrm{H}_{2} \mathrm{O} / \mathrm{GJ}$, respectively. Therefore, the optimal product distributions of steam cracking lead to better economic performance but worse environmental performance than the product distributions of steam cracking taken from the literature. 


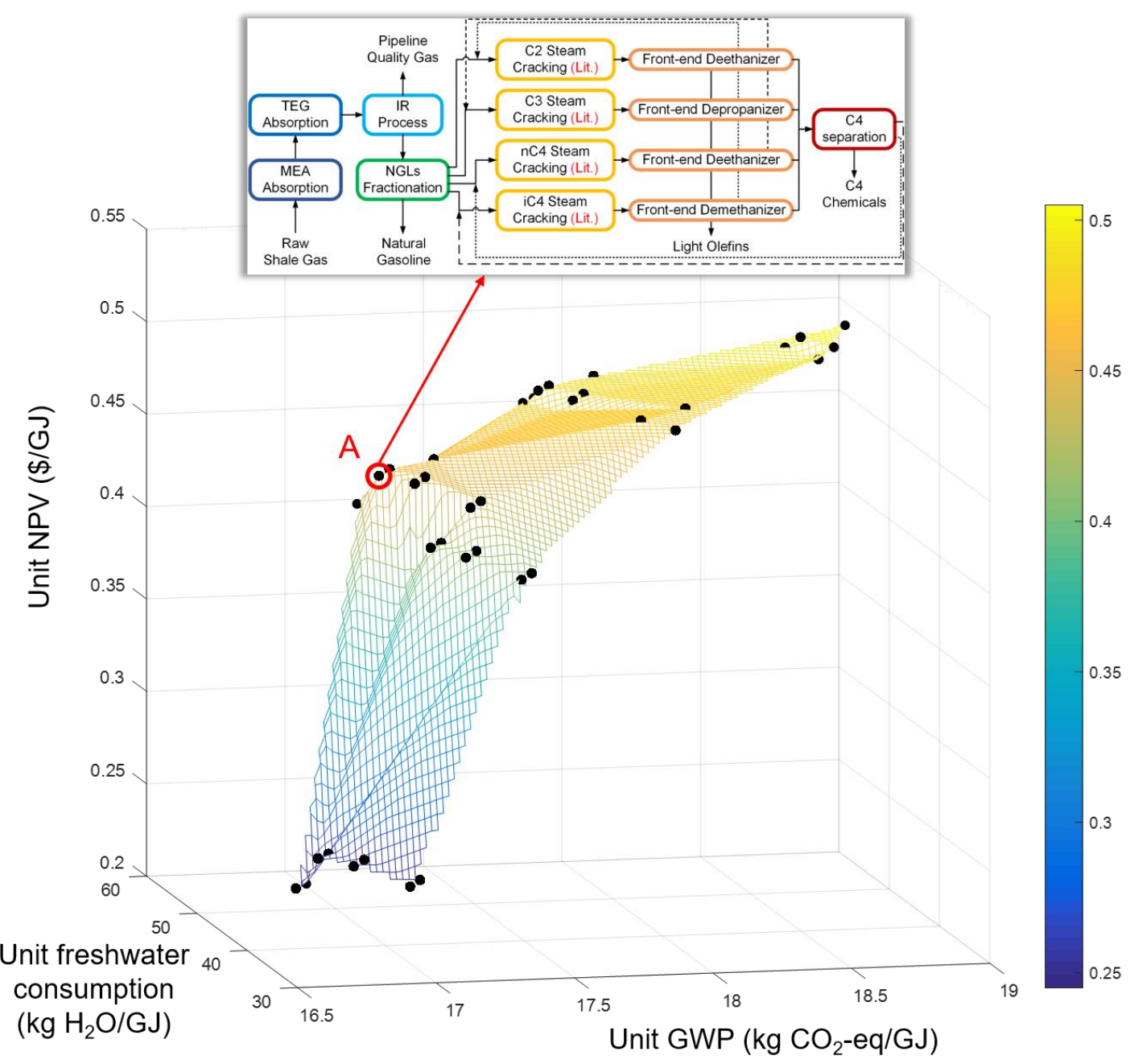

Figure 49. Pareto-optimal surface with the product distributions of steam cracking taken from the literature, showing the trade-offs among the NPV per GJ of raw shale gas, the GWP per GJ of raw shale gas, and the water footprint per GJ of raw shale gas. A good-choice optimal solution and the selected technologies/processes are also presented.

The good-choice optimal solutions A and B consider a TEG absorption process in the dehydration section. Demonstrating a moderate GWP and a moderate water footprint rate simultaneously, the TEG absorption process becomes a balanced option when the unit GWP and the unit water footprint are minimized simultaneously. 


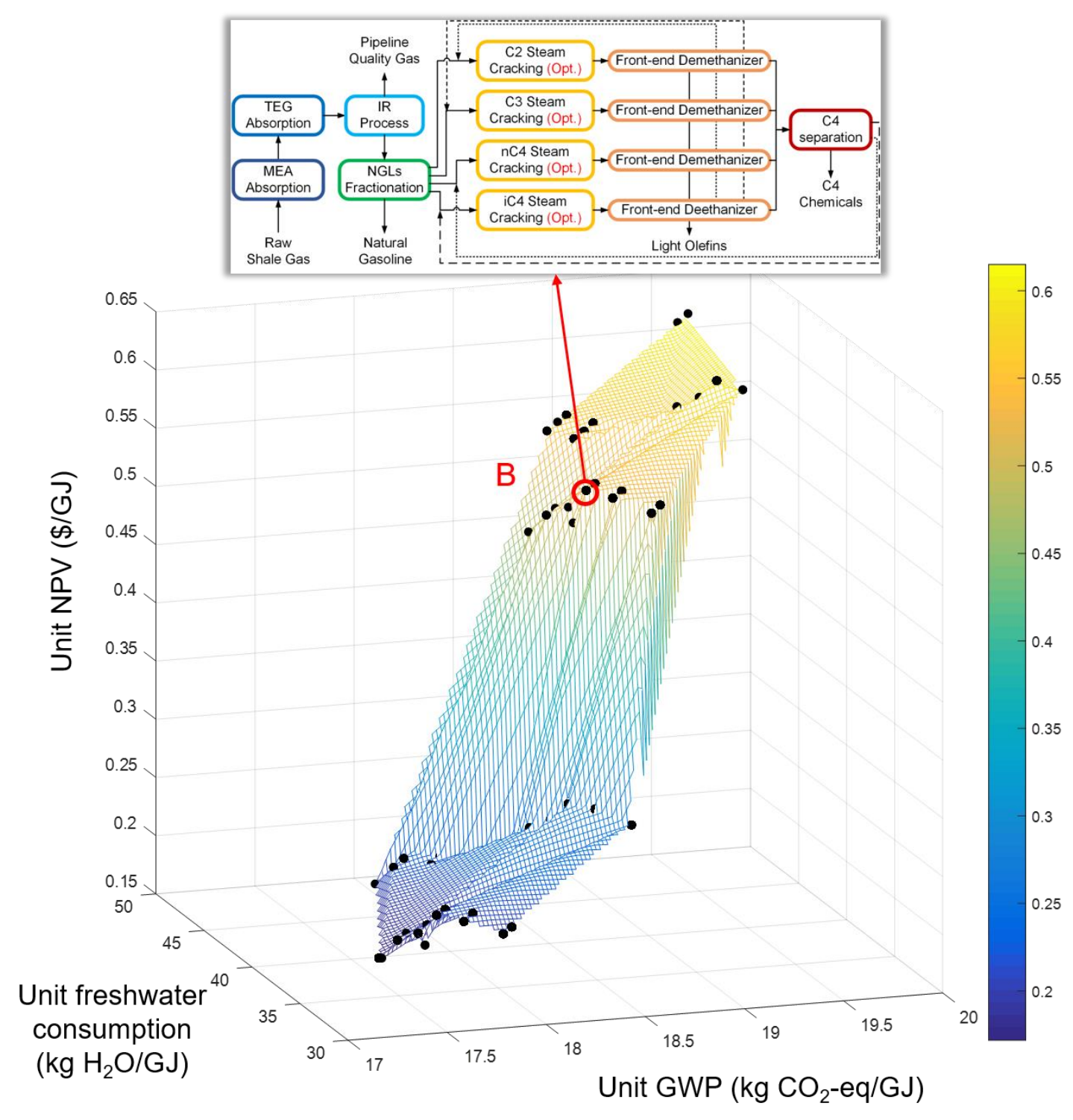

Figure 50. Pareto-optimal surface with the optimal product distributions of steam cracking, showing the trade-offs among the NPV per GJ of raw shale gas, the GWP per GJ of raw shale gas, and the water footprint per GJ of raw shale gas. A good-choice optimal solution and the selected technologies/processes are also presented.

In the hydrocarbons conversion section, only steam cracking processes are selected by the good-choice solutions. However, the other hydrocarbons conversion technologies/processes can be favorable if only one objective function is 
considered. To maximize the unit NPV, the optimal process flowsheet selects catalytic dehydrogenation of propane and $n$-butane, because the product distributions of these reactions are more profitable than those of the corresponding steam cracking reactions. 


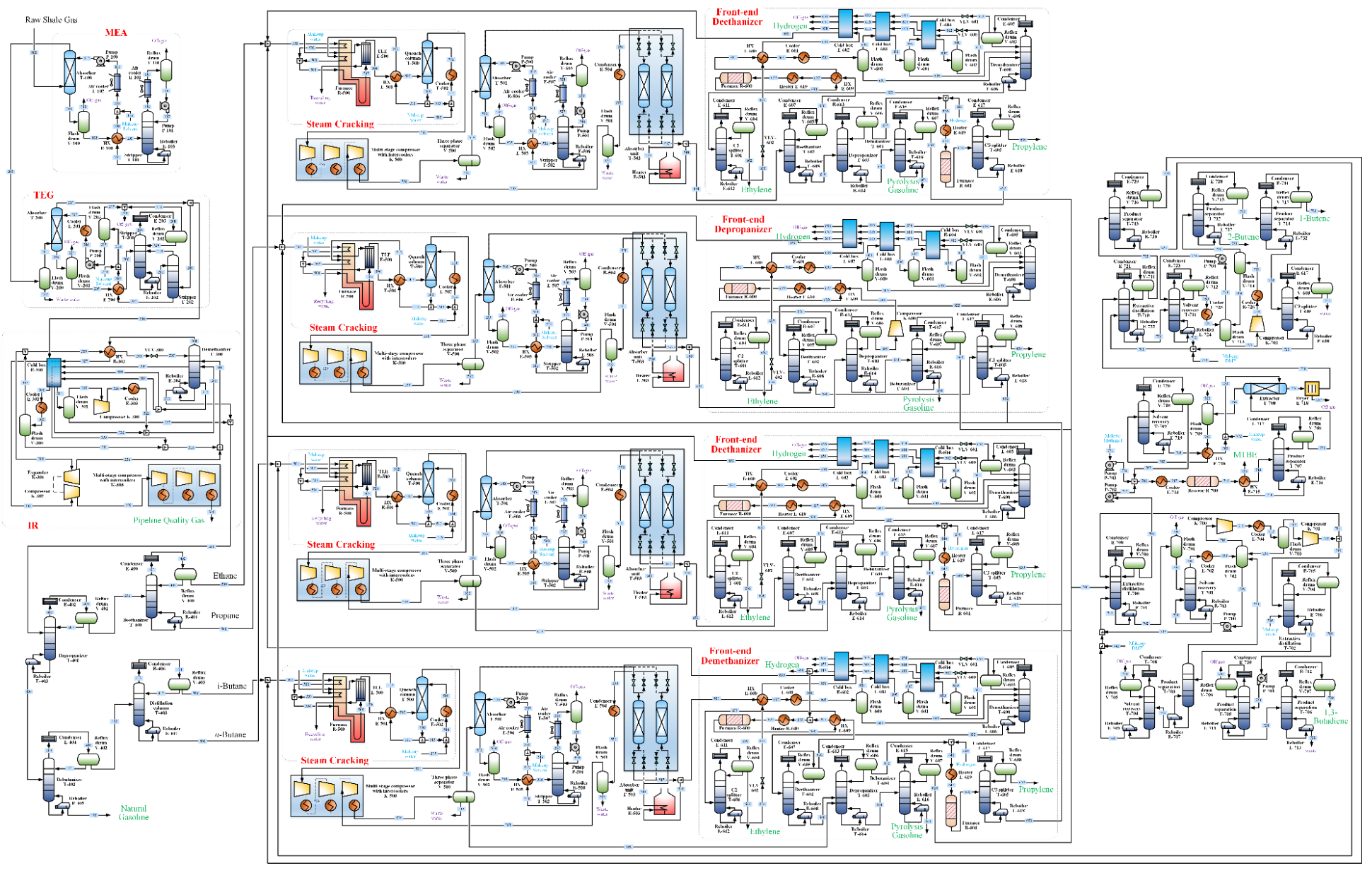

Figure 51. Optimal process flowsheet of the good-choice optimal solution A in Figure 49. 


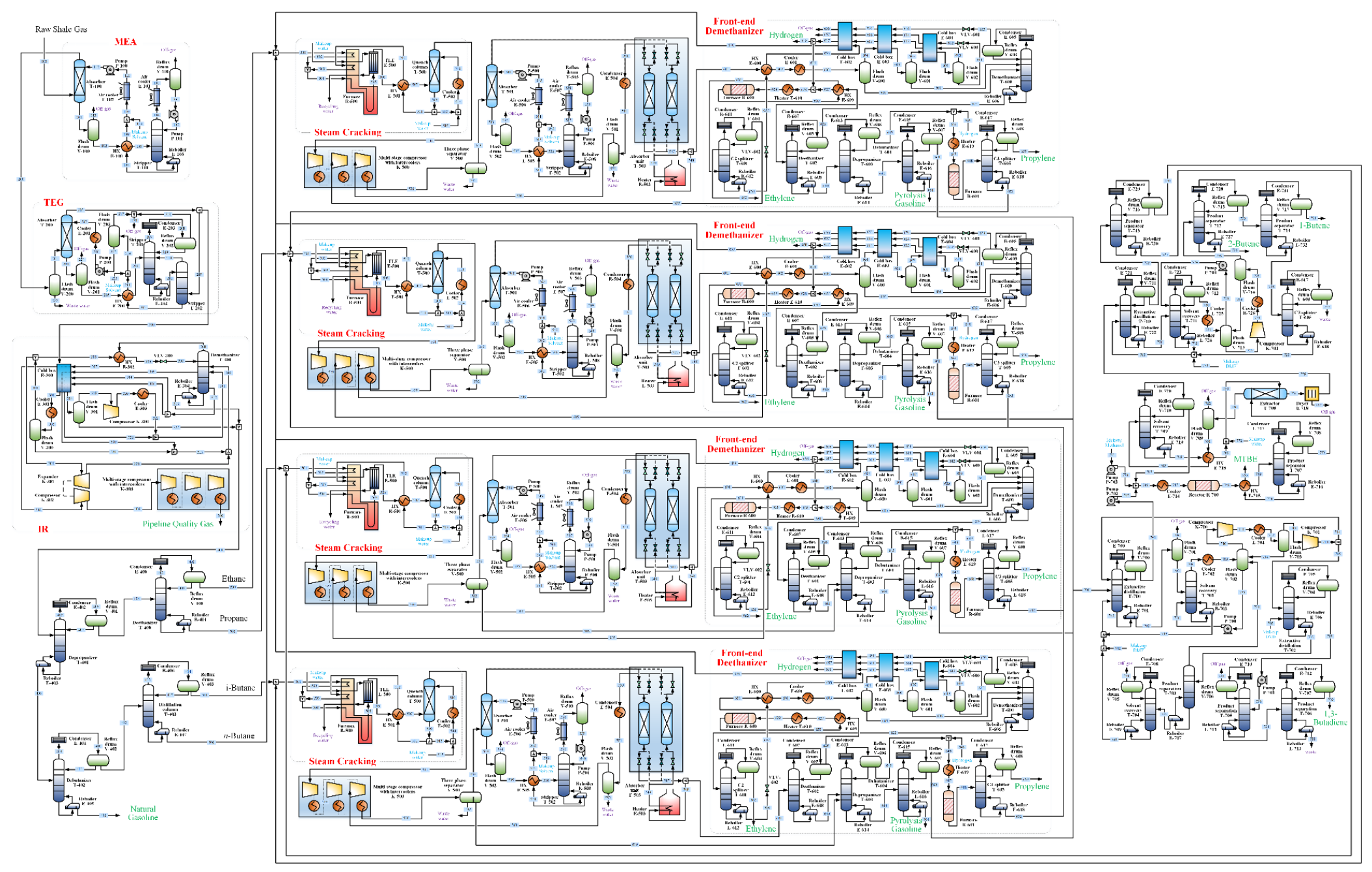

Figure 52. Optimal process flowsheet of the good-choice optimal solution B in Figure 50. 


\subsubsection{Cost and environmental impacts breakdowns}

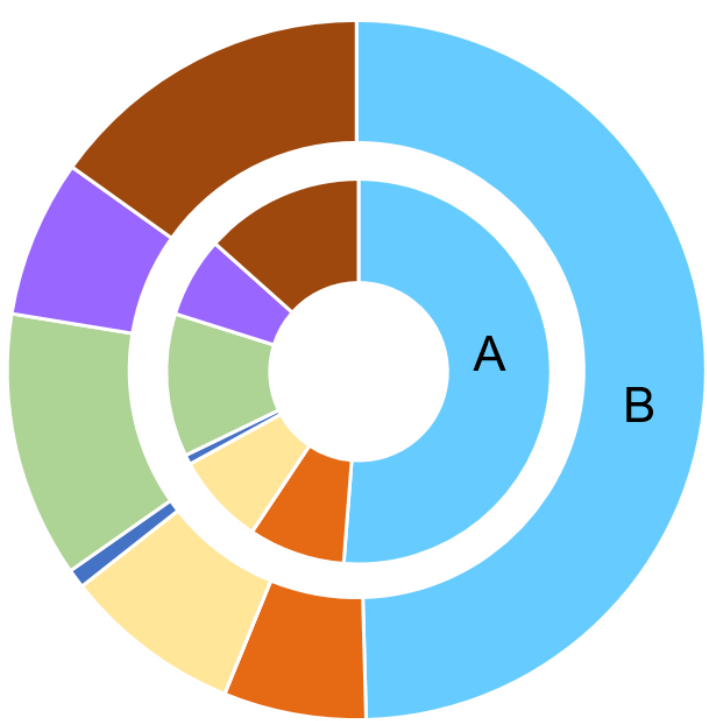

$\square$ Feedstock cost

$\square$ Wastewater treatment cost

- PT\&I

$\square$ Annualized total capital investment
- Utility cost

O\&M cost

General expense

- Income tax

Figure 53. Breakdowns of the OPEX and the annualized CAPEX for the good-choice optimal solution A in Figure 49 and the good-choice optimal solution B in Figure 50.

To better understand the optimal process designs, we present the breakdowns of the OPEX and the annualized CAPEX of the good-choice optimal solutions A and $\mathrm{B}$ in Figure 53. The annualized CAPEX is calculated with a project lifetime of 20 years and a discount rate of $10 \%[14,19,307]$. The capital cost of each technology of the good-choice optimal solutions A and B is presented in Table 10. The dominant contributor in Figure 53 is the feedstocks cost and around 97\% of the feedstock cost is spent in purchasing the raw shale gas. An OPEX item that 
deserves additional attention is the income tax, which is evaluated as a percentage of the difference between the revenue and all other OPEX items. If a fixed amount of raw shale gas, for example $1 \mathrm{GJ}$, is fed to the integrated shale gas processing and chemical manufacturing process, the feedstock cost will be almost the same for all the optimal process designs. Therefore, a higher ratio of the income tax to the feedstock cost indicates a higher profit per year and finally a higher unit NPV. The ratio of the income tax to the feedstock cost for the optimal solution $B$ is 0.31 , while the ratio for the optimal solution A is 0.26 . Therefore, the optimal process design for the optimal solution B is more economically competitive.

Table 10. Breakdown of CAPEX for optimal solution A and optimal solution B.

\begin{tabular}{|c|c|c|c|}
\hline & Technologies/Processes & $\begin{array}{r}\text { Costs for } \\
\text { optimal } \\
\text { solution A } \\
(\mathrm{MM} \$)\end{array}$ & $\begin{array}{r}\text { Costs for } \\
\text { optimal } \\
\text { solution B } \\
(\mathrm{MM} \$)\end{array}$ \\
\hline \multirow{12}{*}{$\begin{array}{l}\text { Direct } \\
\text { cost }\end{array}$} & MEA Absorption & 1.17 & 1.17 \\
\hline & TEG Absorption & 1.78 & 1.78 \\
\hline & IR Process & 18.58 & 18.58 \\
\hline & NGLs Fractionation & 5.20 & 5.20 \\
\hline & C2 Steam Cracking & 54.31 & 54.31 \\
\hline & C3 Steam Cracking & 30.68 & 30.68 \\
\hline & $n C 4$ Steam Cracking & 11.29 & 11.29 \\
\hline & ¡C4 Steam Cracking & 5.84 & 5.84 \\
\hline & Light olefins separation for C2 & 16.93 & 23.78 \\
\hline & Light olefins separation for C3 & 20.23 & 24.29 \\
\hline & $\begin{array}{l}\text { Light olefins separation for } \\
n \mathrm{C} 4\end{array}$ & 4.16 & 9.07 \\
\hline & $\begin{array}{l}\text { Light olefins separation for } \\
\text { iC4 }\end{array}$ & 2.55 & 2.03 \\
\hline
\end{tabular}


C4 Separation

Steam Turbine

Total direct cost

Indirect cost

Fixed capital investment

Working capital

Total capital investment
11.59

11.24

195.55

68.44

263.99

13.20

277.19
14.56

12.62

215.20

75.32

290.52

14.53

305.05

The breakdowns of the GWP and the water footprint of the good-choice optimal solutions A and B are presented in Figure 54. The GWP in the superstructure optimization model can be classified by the sources of emissions. The GWP associated with direct emissions account for the off-gas from the integrated shale gas processing and chemical manufacturing process, while the GWP associated with indirect emissions account for the off-gas from feedstock extraction, heating utilities production, cooling utilities production, electricity production, and wastewater treatment. Since the GWP associated with wastewater treatment is several orders of magnitute smaller than the GWP associated with other sources, wastewater treatment is not included in the breakdowns of the GWP. For both optimal solution A and optimal solution B, the largest share of GWP comes from feedstocks extraction. Moreover, since producing heating utilities is relatively more energy-intensive than producing cooling utilities and electricity, heating utilities production causes more GWP than the other two utilities-related contributors. The GWP associated with heating utilities production in the optimal 
solution B is higher than that of the optimal solution A. Therefore, future development of the integrated shale gas processing and chemical manufacturing process can focus on improving the energy utilitzaiton efficiency to reduce the total GWP.

The breakdowns of the water footprint are also displayed in Figure 54. The water footprint in this chapter consists of direct water consumption in the integrated shale gas processing and chemical manufacturing process, indirect water consumption in feedstock extraction and utilities production, and indirect water production in wastewater treatment. However, as the water footprint of the wastewater treatment process is negative, it is excluded in the breakdowns. Among the considered contributors, cooling utilities consume much more water than others, because a substantial amount of water evaporates to reduce the temperature of the remaining cooling water. To ensure the cooling quality, makeup water is added to the cooling system. Given that cooling is needed for all the reactors and distillation columns, makeup water becomes the dominant consumer of water within the entire system. The water footprint for utilities production in the optimal process design of the optimal solution A is smaller than that of the optimal solution $\mathrm{B}$, but the direct water consumption in the optimal process design of optimal solution A is higher than that of the optimal solution B. To save more 
water, future research may be focused on improving the efficiency of the cooling water system.
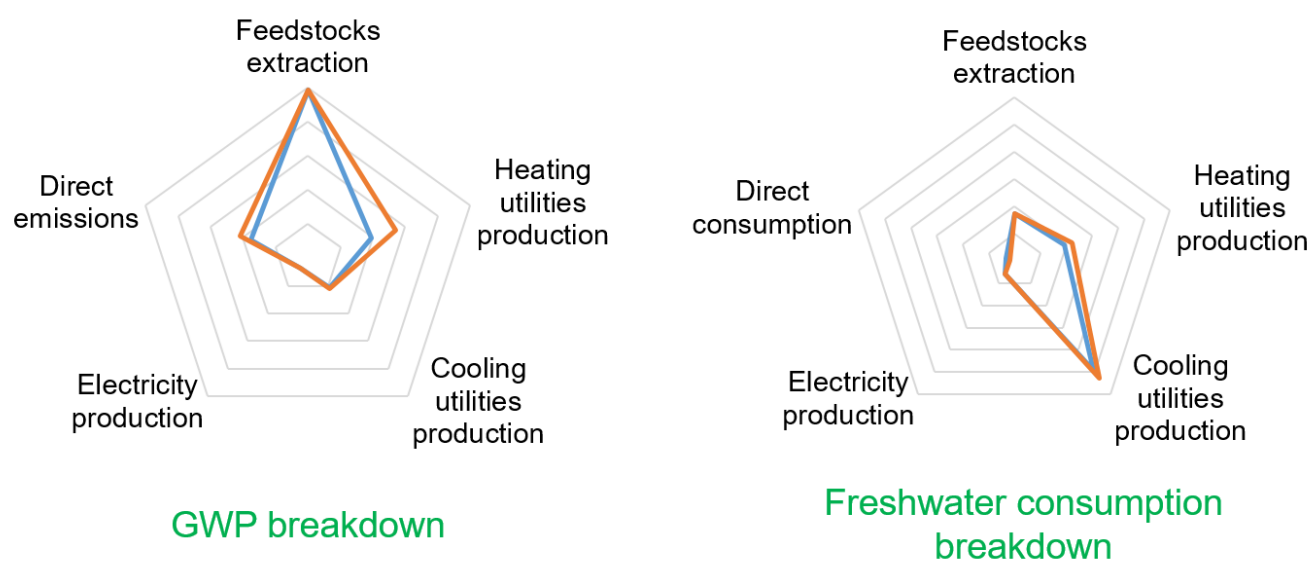

- Solution A - Solution B

Figure 54. Breakdowns of the GWP and the water footprint of the good-choice optimal solution A in Figure 49 and the good-choice optimal solution B in Figure 50.

\subsubsection{Result validation by a whole-process simulation}

A key assumption of the superstructure model is that the mass and energy balance relationships scale linearly. To develop the superstructure optimization model for process synthesis of the integrated shale gas processing and chemical manufacturing process, a set of simulation models of the involved processes are first developed separately with fixed operating conditions. Based on the simulation results, linear mass balance and energy balance constraints are developed and included in the superstructure optimization model. However, this assumption is not stringent in a whole-process simulation, where the connected unit operations are powered by nonlinear mass and energy relationships and complex phase 
equilibrium constraints. Therefore, it is worth examining how much the flow rates in the optimal solution deviate from corresponding simulation results. Based on the optimal process design of the optimal solution $\mathrm{B}$, we perform a whole-process simulation and summarize the flow rates of the nine products from both methods in Figure 55. The relative differences between the optimization results and the simulation results range from $0.05 \%$ to $5.78 \%$, while the absolute differences range from $0 \mathrm{t} / \mathrm{h}$ to $0.07 \mathrm{t} / \mathrm{h}$. Higher relative differences correspond to the products with considerably small absolute differences.

Overall, the optimization results agree well with the whole-process simulation results. Note that the validation results do not suggest that the linear process models represent good approximations to the complex models in all circumstances. In fact, it is important to set the operating conditions of the simulation models for different alternatives processes in the superstructure as close to the optimal solution as possible, and validate the solution iteratively with whole-process simulations. As mentioned previously, the optimization results can converge to the simulation results in finite iterations, because there are finite process alternatives/designs in the superstructure, and the parameters of one process design are updated in each iteration. By enumerating the process alternatives/designs, the 
optimization can finally return the same results with the corresponding wholeprocess simulation.
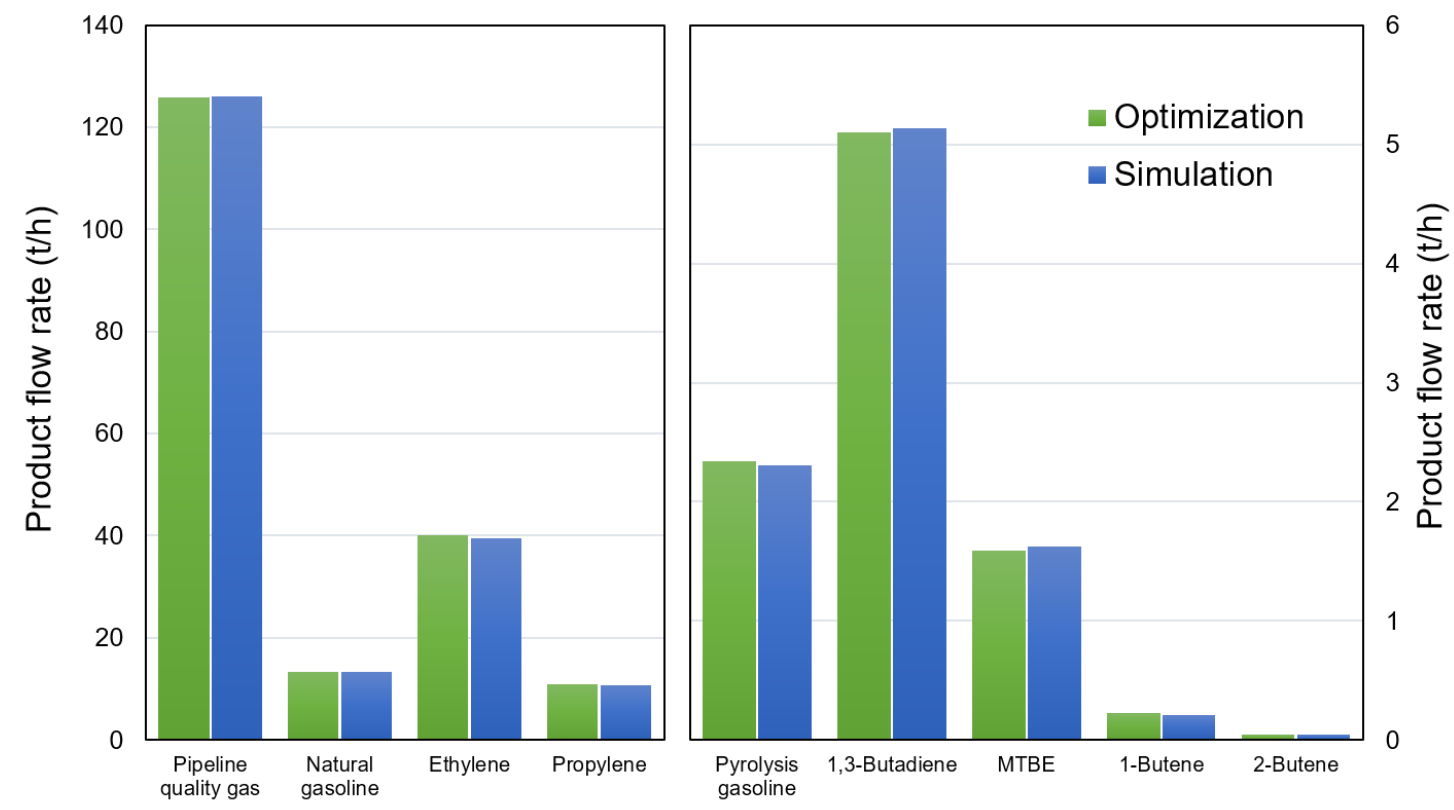

Figure 55. Comparison of the product flow rates for the optimal solution B based on optimization and the corresponding whole-process simulation.

\subsubsection{Sensitivity analysis}

The parameters considered in the superstructure optimization problem can be classified into two groups: the parameters relevant to markets and the parameters irrelevant to markets. The market related parameters, such as prices, are volatile in most circumstances. Therefore, even though we apply the most recent values of these parameters for the Pareto-optimal surfaces, it is worth investigating how these parameters influence the optimization results. Figure 56 and Figure 57 present how the optimal economic objective function values of the most 
economically competitive optimal solutions change when the market related parameters are allowed to deviate $20 \%$ from their current values. It is noted that for the product distributions of steam cracking taken from the literature, the most influential market related parameter is the price of raw shale gas. A $20 \%$ increase in the price of raw shale gas reduces $29 \%$ of the highest NPV, while a $20 \%$ decline raises $29 \%$ of the highest NPV. The prices of feedstocks and utilities are in a negative correlation with the highest NPV, while the prices of products are in a positive correlation with the highest NPV. One exception is the price of electricity, because the optimal design generates surplus electricity and treats electricity as a product instead of a feedstock/utility. The influence of market related parameters is similar when the optimal product distributions of steam cracking are considered. In Figure 57, the price of raw shale gas causes the largest change in the highest NPV. Compared with Figure 56, the influence of the price of pipeline quality gas and the price of ethylene is less significant, while the influence of the price of 1,3butadiene is more pronounced in Figure 57. 


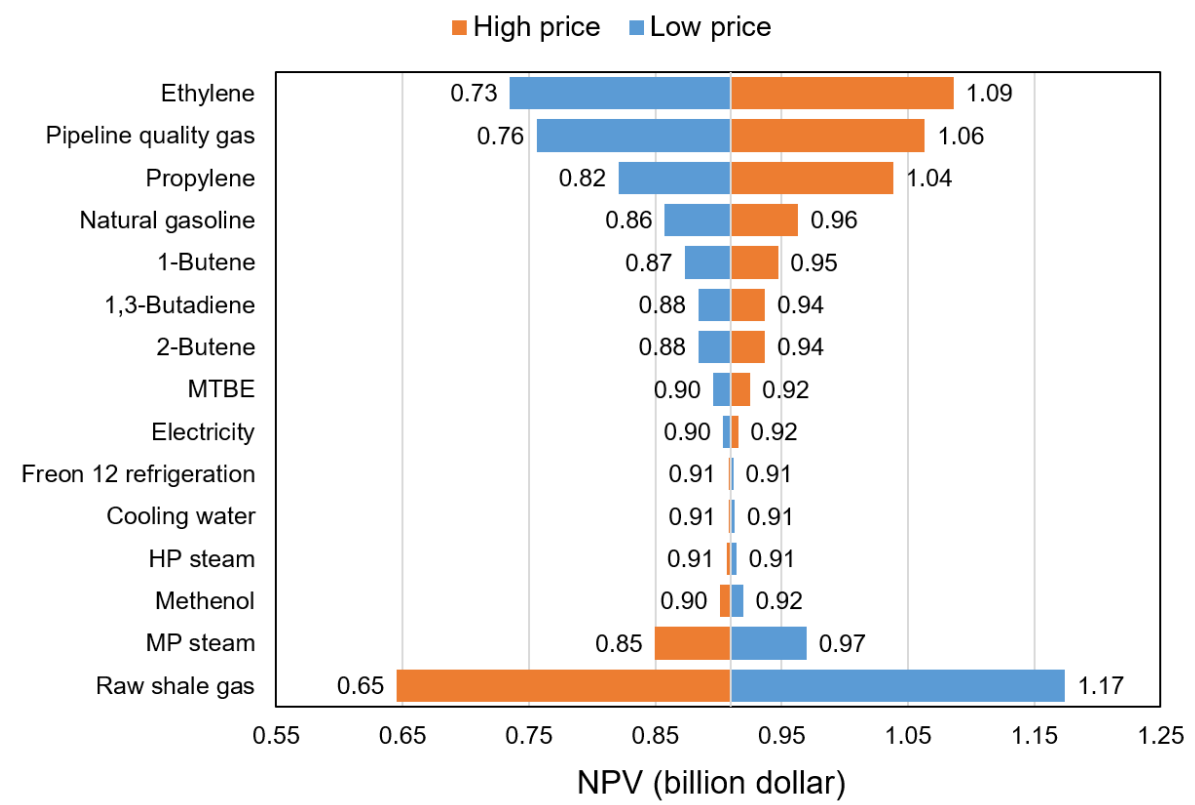

Figure 56. Sensitivity analysis results for the most economically competitive optimal solution with the product distributions of steam cracking taken from the literature. The high price and the low price correspond to $120 \%$ and $80 \%$ of the current price, respectively.

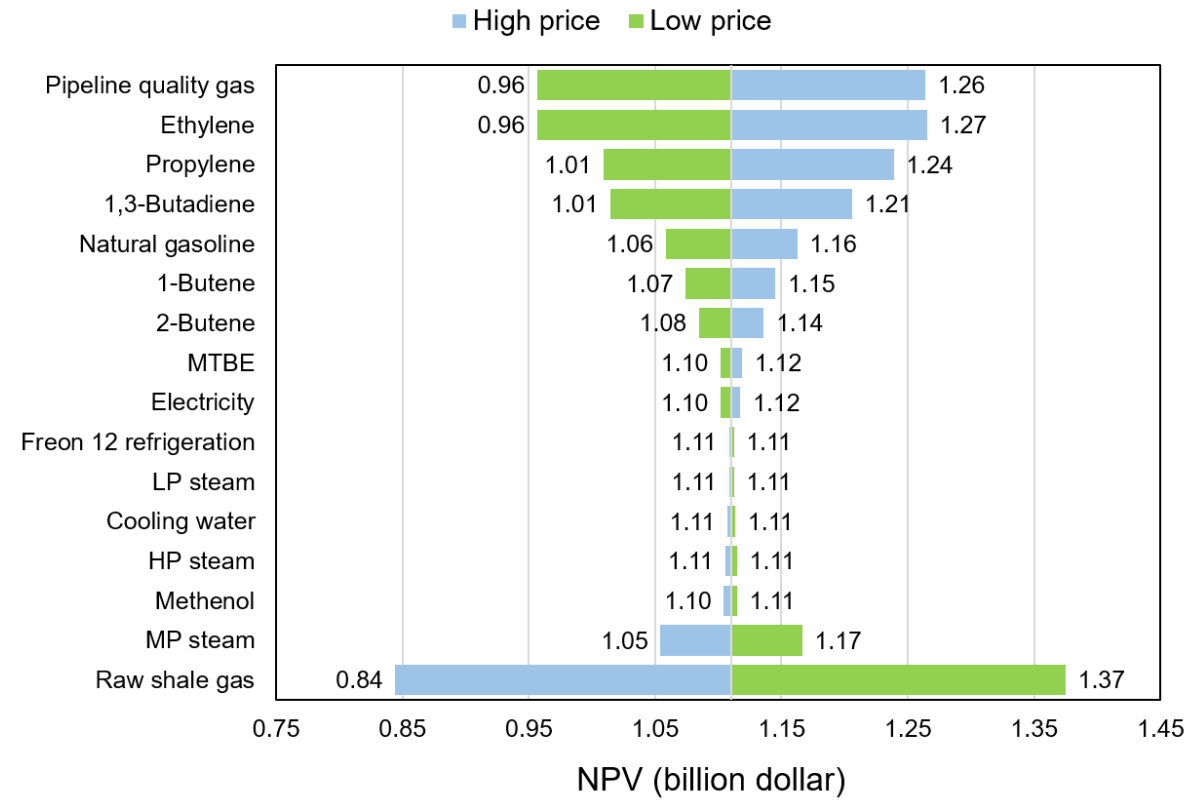

Figure 57. Sensitivity analysis results for the most economically competitive optimal solution with the optimal product distributions of steam cracking. The high price and the low price correspond to $120 \%$ and $80 \%$ of the current price, respectively. 


\subsubsection{Computational performance}

The product distribution optimization model for steam cracking of ethane consists of 31 continuous variables and 46 constraints. These constraints include 11 ODEs for the continuity equations. A few more variables, constraints, and ODEs are included in the product distribution optimization models for steam cracking of heavier hydrocarbons because more species and kinetic equations are involved. When solving the ODEs, the solver is set to return the solutions evaluated at every $0.1 \mathrm{~m}$. All the product distribution problems for steam cracking can be solved efficiently in a few seconds.

The superstructure optimization model consists of 43 integer variables, 164,248 continuous variables, and 290,987 constraints. The global optimization algorithm provides an efficient tool for solving the large-scale MINLP problem for the process synthesis of the integrated shale gas processing and chemical manufacturing process.

Table 11. Computation performance for 6 instances on the Pareto optimal curve with the optimal product distributions of steam cracking using BARON 17.1, SCIP 3.2, and the tailored global optimization algorithm.

\begin{tabular}{|c|c|c|c|c|c|c|c|}
\hline \multirow[b]{2}{*}{$\begin{array}{c}\text { No } \\
\text {. }\end{array}$} & \multicolumn{2}{|c|}{ BARON 17.1} & \multicolumn{2}{|c|}{ SCIP 3.2} & \multicolumn{3}{|c|}{ Global optimization algorithm } \\
\hline & $\begin{array}{c}\text { Unit NPV } \\
(\$ / G J)\end{array}$ & $\begin{array}{c}\text { CPU } \\
\text { time }(s)\end{array}$ & $\begin{array}{c}\text { Unit NPV } \\
(\$ / G J)\end{array}$ & $\begin{array}{c}\text { CPU time } \\
(\mathrm{s})\end{array}$ & $\begin{array}{c}\text { Unit NPV } \\
(\$ / G J)\end{array}$ & Iter. & $\begin{array}{c}\text { CPU time } \\
(\mathrm{s})\end{array}$ \\
\hline 1 & $\mathrm{~N} / \mathrm{A}^{\mathrm{a}}$ & 3,600 & $\begin{array}{c}\text { Loc. } \\
\text { Infeas. }{ }^{\mathrm{b}}\end{array}$ & 3 & 0.24 & 2 & 2 \\
\hline 2 & $\mathrm{~N} / \mathrm{A}^{\mathrm{a}}$ & 3,600 & $0.26^{c}$ & 3,600 & 0.44 & 4 & 7 \\
\hline 3 & $\mathrm{~N} / \mathrm{A}^{\mathrm{a}}$ & 3,600 & 0.50 & 9 & 0.50 & 4 & 6 \\
\hline
\end{tabular}




\begin{tabular}{llllclll}
4 & $\mathrm{~N} / \mathrm{A}^{\mathrm{a}}$ & 3,600 & 0.17 & 5 & 0.17 & 2 & 4 \\
5 & $\mathrm{~N} / \mathrm{A}^{\mathrm{a}}$ & 3,600 & 0.49 & 9 & 0.49 & 2 & 3 \\
6 & $\mathrm{~N} / \mathrm{A}^{\mathrm{a}}$ & 3,600 & 0.61 & 33 & 0.61 & 4 & 7 \\
\hline
\end{tabular}

a. No upper and low bound information is returned before the program reaches the computation time limit of 1 hour.

b. "Loc. Infeas." denotes "locally infeasible".

c. A feasible solutions is returned before the program reaches the computation time limit.

To demonstrate the computational performance, the proposed superstructure optimization problem is solved by BARON 17.1, SCIP 3.2, and the global optimization algorithm, respectively. Table 11 summarizes the computational results of solving six MINLP problems with the optimal product distributions of steam cracking. The optimal solutions of these instances correspond to six points on the Pareto-optimal curve with the optimal product distributions of steam cracking. In all the instances, BARON 17.1 proceeds to the local search phase in a few seconds and fails to return any feasible solution within 1 hour. Another global optimizer SCIP 3.2 shows better computational performance than BARON 17.1, because it returns the optimal solutions of Instances 3-6 and a suboptimal solution of Instance 2. However, SCIP 3.2 fails to converge in Instances 1 within 1 hour. In contrast, the tailored global optimization algorithm returns the optimal solutions in all the instances within 5 iterations and 10 CPUs. Overall, the global optimization algorithm is more efficient than general-purpose MINLP solvers for solving the resulting MINLP problems. 


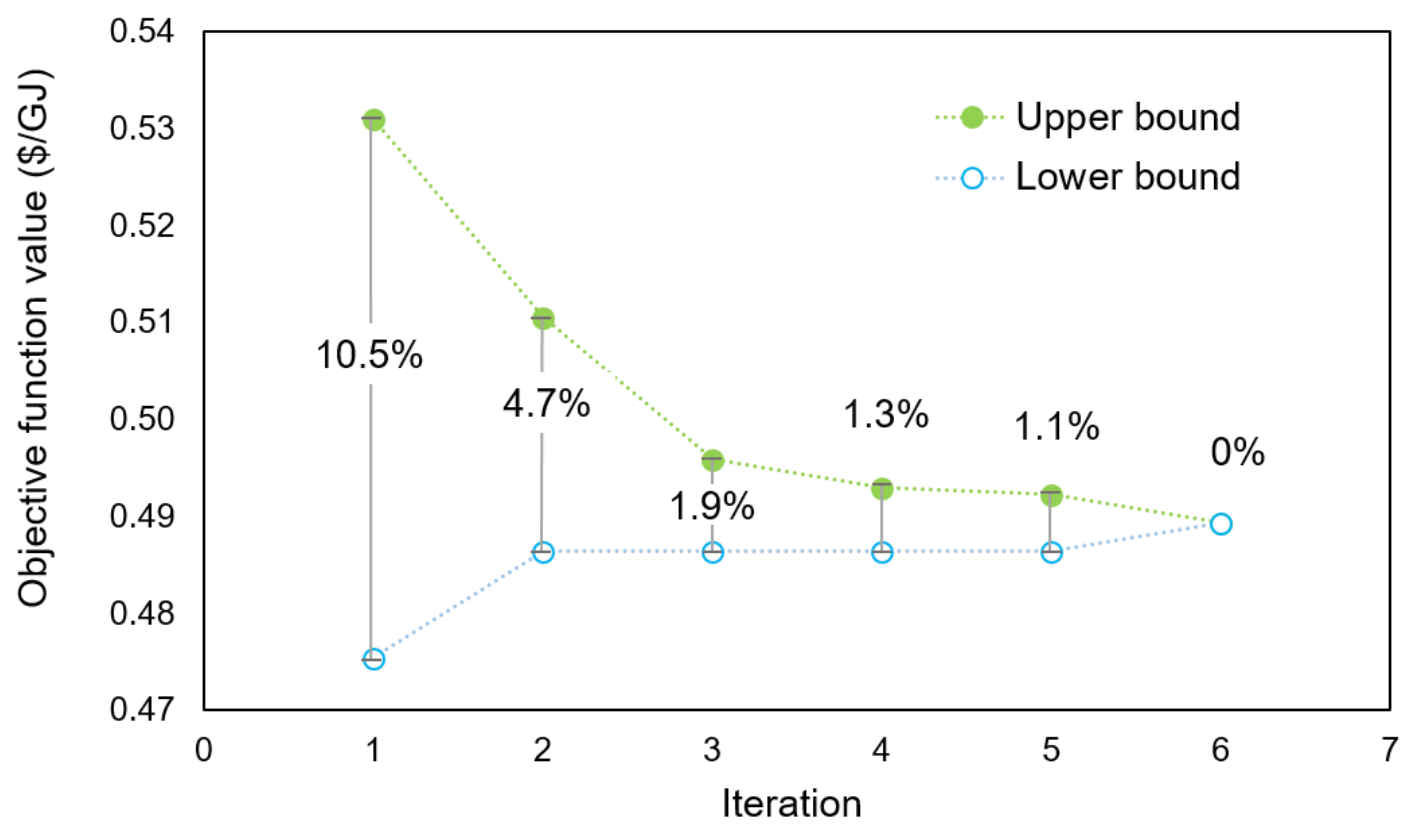

Figure 58. Upper and lower bounds in each iteration of the global optimization algorithm for solving the superstructure optimization problem in Instance 5 in Table 11.

In Figure 58, we present the lower and upper bounds of each iteration of the global optimization algorithm when solving the MINLP problem in Instance 5 in Table 11. Only 6 iterations are needed to reduce the relative optimality gap to $0 \%$. The best upper bound decreases as the number of iterations increases, and more feasible solutions are added to the piecewise linear approximation functions. The best lower bound keeps increasing until the upper bound and lower bound are close enough. It is noted that the relative optimality gap between the best upper bound and lower bound reduces rapidly in the first three iterations, showing that the algorithm can add new partition points automatically and efficiently for improving the accuracy of the approximation functions. 


\subsection{Summary}

We proposed a novel process synthesis framework that combines product distribution optimization of chemical reactions and superstructure optimizaiton of the process flowsheet. The product distributions of critical chemical reactions in a superstructure were first optimized to maximize the profits of the effluent products. With the optimal product distributions of the chemical reactions, extensive process simulations were performed to collect high-fidelity process data. Next, the simulation results were used to develop an MINLP model for superstructure optimization. In the last step, the optimal process design was validated by a whole-process simulation. The proposed framework was illustrated by an application to an integrated shale gas processing and chemical manufacturing process. The proposed superstructure included 51,840 alternative possible process designs and consisted of eight sections, namely acid gas removal, dehydration, NGLs recovery, NGLs separation, hydrocarbons conversion, light olefins separation, C4 separation, and acid gas disposal. For the steam cracking processes in the hydrocarbons conversion section, several dynamic optimization models were developed to maximize the profits of producing various chemicals from hydrocarbons. Based on the optimal product distributions of steam cracking, a set of simulation models were developed for all the involved processes in the 
superstructure. A multiobjective MINLP model was developed to address the sustainable design and synthesis of the integrated shale gas processing and chemical manufacturing process. To solve the resutling MINLP problem efficiently, we employeed a tailored global optimization algorithm that integrates the inexact parametric algorithm and the branch-and-refine algorithm. A goodchoice solution was identified on the resulting Pareto-optimal surface with the optimal product distributions of steam cracking. The process design selected MEA absorption, TEG absorption, IR process, NGLs separation, steam cracking of ethane with front-end demethanizer, steam cracking of propane with front-end demethanizer, steam cracking of $n$-butane with front-end demethanizer, steam cracking of $i$-butane with front-end deethanizer, and C4 separation. The highest NPV per GJ of raw shale gas was higher when the optimal product distributions of steam cracking were used, while the minimum GWP and water footprint per GJ of raw shale gas were lower when the product distributions of steam cracking taken from the literature were used. A sensitivity analysis showed that the most influencial market related parameter in the superstructure optimization problem was the price of raw shale gas. The tailored global optimization algorithm was more efficient than general-purpose solvers when handling the proposed MINLP model. 


\subsection{Appendix A: Model formulation of the product distribution optimization problem for steam cracking of ethane}

$$
\begin{aligned}
& i=1: \mathrm{C}_{2} \mathrm{H}_{6} \rightleftharpoons \mathrm{C}_{2} \mathrm{H}_{4}+\mathrm{H}_{2} \\
& i=2: \quad 2 \mathrm{C}_{2} \mathrm{H}_{6} \rightarrow \mathrm{C}_{3} \mathrm{H}_{8}+\mathrm{CH}_{4} \\
& i=3: \mathrm{C}_{3} \mathrm{H}_{6} \rightleftharpoons \mathrm{C}_{2} \mathrm{H}_{2}+\mathrm{CH}_{4} \\
& i=4: \mathrm{C}_{2} \mathrm{H}_{2}+\mathrm{C}_{2} \mathrm{H}_{4} \rightarrow \mathrm{C}_{4} \mathrm{H}_{6} \\
& i=5: \mathrm{C}_{2} \mathrm{H}_{4}+\mathrm{C}_{2} \mathrm{H}_{6} \rightarrow \mathrm{C}_{3} \mathrm{H}_{6}+\mathrm{CH}_{4}
\end{aligned}
$$

According to a kinetic model [272], the above five reactions take place simultaneously during steam cracking of ethane. In the corresponding product distribution optimization problem, the reactions are indexed by $i$ and the species are indexed by j. $\mathrm{C}_{2} \mathrm{H}_{6}, \mathrm{C}_{2} \mathrm{H}_{4}, \mathrm{H}_{2}, \mathrm{CH}_{4}, \mathrm{C}_{3} \mathrm{H}_{6}, \mathrm{C}_{3} \mathrm{H}_{8}, \mathrm{C}_{2} \mathrm{H}_{2}$, and $\mathrm{C}_{4} \mathrm{H}_{6}$ correspond to $j=1-8$, respectively. The rates of reaction $R_{i}$ are defined in Eqs. (1.A24)-(1.A28), respectively. $K_{i}$ is the reaction constant of reaction $i, F_{j}$ is the flow rate of species $j$, $F t$ is the total flow rate of the gas mixture, $P$ is pressure, $T$ is temperature, $r c 1$ is the gas constant, $k_{c l}$ and $k_{c 5}$ are two equilibrium constants.

$$
\begin{aligned}
& R_{l}=K_{1} \cdot\left[\frac{F_{1}}{F t} \cdot \frac{P}{r c l \cdot T}-\frac{F_{2} \cdot F_{3}}{F t^{2} \cdot k_{C_{1}}}\left(\frac{P}{r c l \cdot T}\right)^{2}\right] \\
& R_{2}=K_{2} \cdot \frac{F_{1}}{F t} \cdot \frac{P}{r c l \cdot T} \\
& R_{3}=K_{3} \cdot\left[\frac{F_{5}}{F t} \cdot \frac{P}{r c l \cdot T}-\frac{F_{7} \cdot F_{4}}{F t^{2} \cdot k_{C_{5}}}\left(\frac{P}{r c l \cdot T}\right)^{2}\right]
\end{aligned}
$$




$$
\begin{aligned}
& R_{4}=K_{4} \cdot \frac{F_{7} \cdot F_{2}}{F t^{2}} \cdot\left(\frac{P}{r c l \cdot T}\right)^{2} \\
& R_{5}=K_{5} \cdot \frac{F_{1} \cdot F_{2}}{F t^{2}} \cdot\left(\frac{P}{r c l \cdot T}\right)^{2}
\end{aligned}
$$

The reaction constant $K_{i}$ is defined as a function of temperature in Eq. (1.A29), where $a_{i}$ and $e c_{i}$ are the Arrhenius parameters of reaction $i$, and $r c 2$ is the gas constant (differing with $r c 1$ in the unit).

$$
K_{i}=a_{i} \cdot \exp \left(-\frac{e c_{i}}{r c 2 \cdot T}\right), \forall i
$$

The total flow rate $F t$ is defined by Eq. (1.A30), where $F s$ is the flow rate of steam.

$$
F t=\sum_{j} F_{j}+F s
$$

A set of continuity equations for one-dimensional plug flow reactors are used in this model because they can accurately calculate the product yields of steam cracking reactions under a wide range of operating conditions [308]. The mass balance is described by Eq. (1.A31), where $Z$ is the length of the tube, $d$ is the diameter of the tube, and $s_{i, j}$ is the stoichiometric coefficient of species $j$ in reaction i.

$$
\frac{d F_{j}}{d Z}=\frac{\pi \cdot d^{2}}{4} \sum_{i}\left(s_{i, j} \cdot R_{i}\right), \forall j
$$


The energy balance is described by Eq. (1.A32), where $u$ is the overall heat transfer coefficient, $t w$ is the wall temperature of the tube reactor, $d h_{i}$ is the heat of reaction $i, c p_{j}$ is the heat capacity of species $j$ and $c p s$ is the heat capacity of steam.

$$
\frac{d T}{d Z}=\frac{\left[\pi \cdot d \cdot u \cdot(t w-T)-\frac{\pi \cdot d^{2}}{4} \sum_{i}\left(R_{i} \cdot d h_{i}\right)\right]}{\sum_{i}\left(F_{j} \cdot c p_{j}\right)+F s \cdot c p s}
$$

To ensure the safety of the reactor, the temperature of the gas mixture must be bound in $[t l, t u]$, as enforced by Constraint (1.A33).

$$
t l \leq T \leq t u
$$

The momentum balance is described by Eq. (1.A34), where $M t$ is molecular weight of the gas mixture as defined in Eq. (1.A35), $F r$ is the friction factor as defined in Eq. (1.A36), and $G$ is the total mass flux as defined in Eq. (1.A37). Reynolds number $R e$ is defined in Eq. (1.A38), where $\rho$ and $\mu$ are the density and viscosity of the gas mixture, respectively.

$$
\begin{aligned}
& \frac{d P}{d Z}=\frac{\frac{d}{d Z}\left(\frac{1}{M t}\right)+\frac{1}{M t}\left(\frac{1}{T} \cdot \frac{d T}{d Z}+F r\right)}{\frac{1}{M t \cdot P}-\frac{P}{\alpha \cdot G^{2} \cdot r c l \cdot T}} \\
& M t=\frac{\sum_{j}\left(F_{j} \cdot m_{j}\right)+F s \cdot m s}{F t} \\
& F r=0.092 \times \frac{R e^{-0.2}}{d}
\end{aligned}
$$




$$
\begin{aligned}
& G=\frac{4}{\pi \cdot d^{2}}\left[\sum_{j}\left(F_{j} \cdot m_{j}\right)+F s \cdot m s\right] \\
& R e=4 \times \frac{F t \cdot \rho \cdot r c l \cdot T}{\pi \cdot d \cdot \mu \cdot P}
\end{aligned}
$$

Similar to the safety requirement for temperature, the pressure of the gas mixture must be bound in $[p l, p u]$, as enforced by Constraint (1.A39).

$$
p l \leq P \leq p u
$$

The initial values of the flow rates, temperature, and pressure are provided by Eqs. (1.A40)-(1.A42). The ratio of the flow rate of ethane to that of steam is bound in $[r l, r u]$, as enforced by Constraint (1.A43).

$$
\begin{aligned}
& F_{j, Z=0}=f O_{j}, \forall j \\
& T_{Z=0}=t O \\
& P_{Z=0}=p O \\
& F s_{Z=0} \cdot r l \leq F_{l, Z=0} \leq F s_{Z=0} \cdot r u
\end{aligned}
$$

The revenue is calculated using Eq. (1.A44), where $p r_{j}$ is the price of species $j, J P$ is the set of products, and $Z^{*}$ is the length of the reactor. In Eqs. (1.A45)-(1.A47), investment cost $I C$, feedstock cost $F C$, and utility cost $U C$ are calculated, respectively. ict represents the investment cost per unit length of the reactor; $J F$ is the set of feedstocks; prs is the price of steam; prh is the price of the heating utility.

$$
R E V=\sum_{j \in J P} p r_{j} \cdot F_{j, Z=Z^{*}}
$$




$$
\begin{aligned}
& I C=i c t \cdot Z^{*} \\
& F C=\sum_{j \in J F} p r_{j} \cdot\left(F_{j, Z=0}-F_{j, Z=Z^{*}}\right)+p r s \cdot F s \\
& U C=p r h \cdot \int_{0}^{Z^{*}} \pi \cdot d \cdot u \cdot(t w-T) \cdot d Z
\end{aligned}
$$

Finally, the profit is defined as the difference between the revenue and all the costs in Eq. (1.A48).

$$
P R O=R E V-I C-F C-U C
$$

\subsection{Appendix B: The optimal results of the product distribution optimization problems for steam cracking}

The optimal flow rates of the involved species along the reactors for steam cracking of ethane, propane, $n$-butane, and $i$-butane are presented in Figure B1, Figure B2, Figure B3, and Figure B4, respectively. The optimal product distributions of steam cracking and the product distributions of all the reactions taken from the literature are given in Table B1. The single-pass conversions of the optimal product distributions of steam cracking are lower than those of the corresponding product distributions of steam cracking taken from the literature. However, the optimal product distributions of steam cracking show higher yields of more profitable products. For example, more 1,3-butadiene can be produced from steam cracking of ethane. In contrast, the single-pass conversion of oxidative dehydrogenation of ethane is higher than that of steam cracking of ethane, but a portion of the ethane 
feed is converted to undesired carbon oxides, lowering the ultimate yields of valueadded products. The catalytic dehydrogenation technologies demonstrate higher yields of a certain olefin product and can potentially benefit from a surge in the market price of that product. However, this feature of catalytic dehydrogenation can sometimes become a drawback when the corresponding market undergoes a down turn.

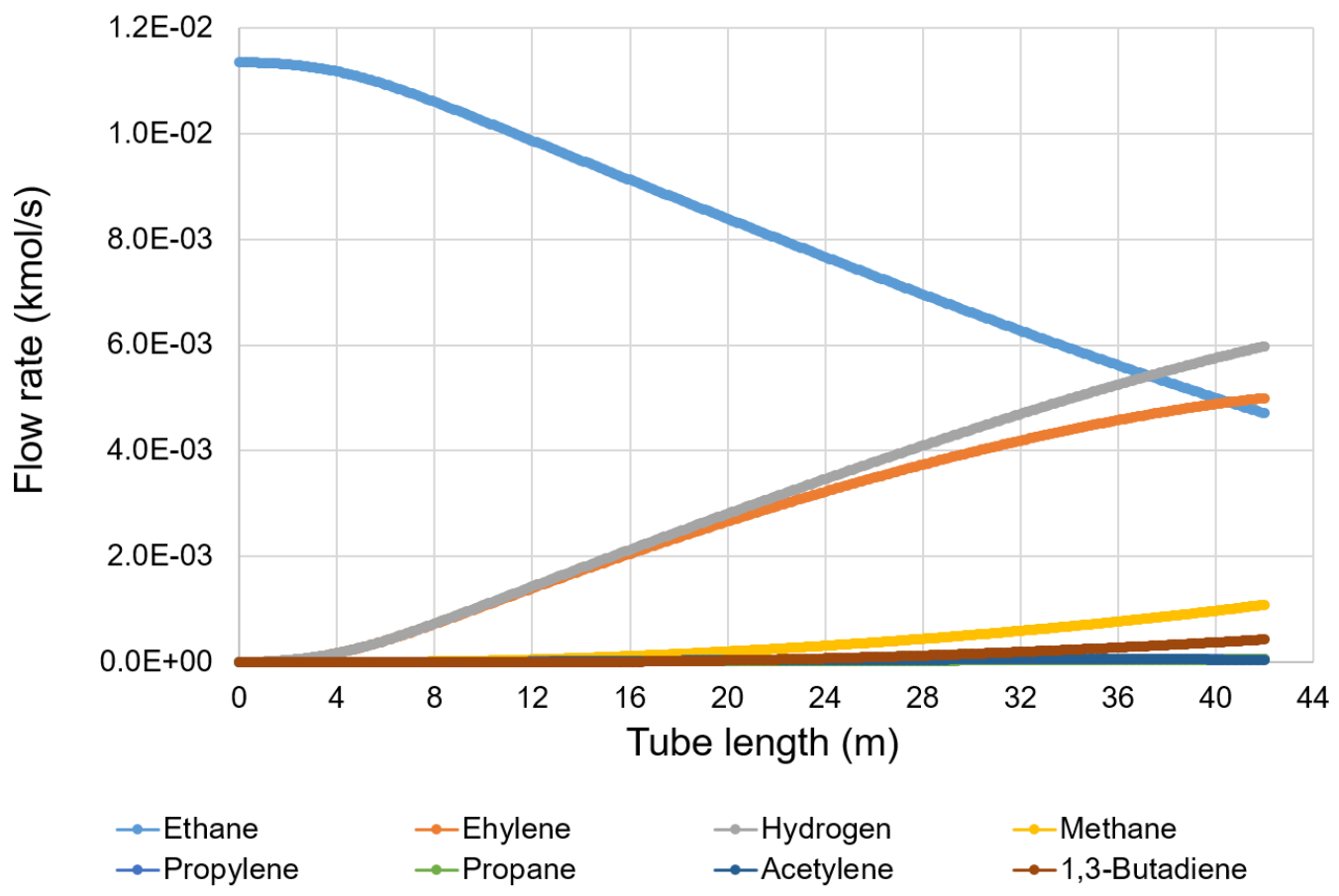

Figure B1. Flow rates of all species along the reactor tube of steam cracking of ethane. 


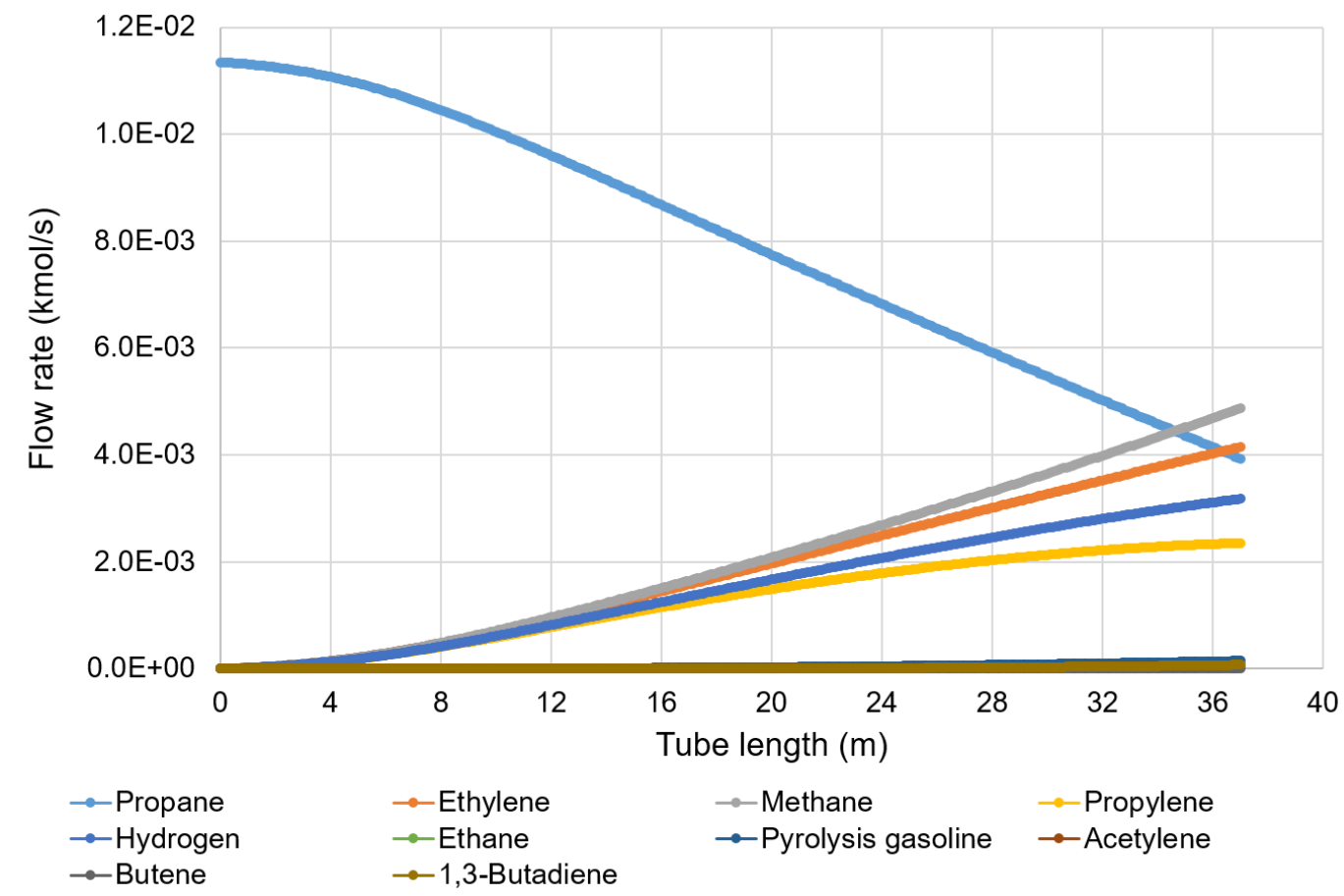

Figure B2. Flow rates of all species along the reactor tube of steam cracking of propane.

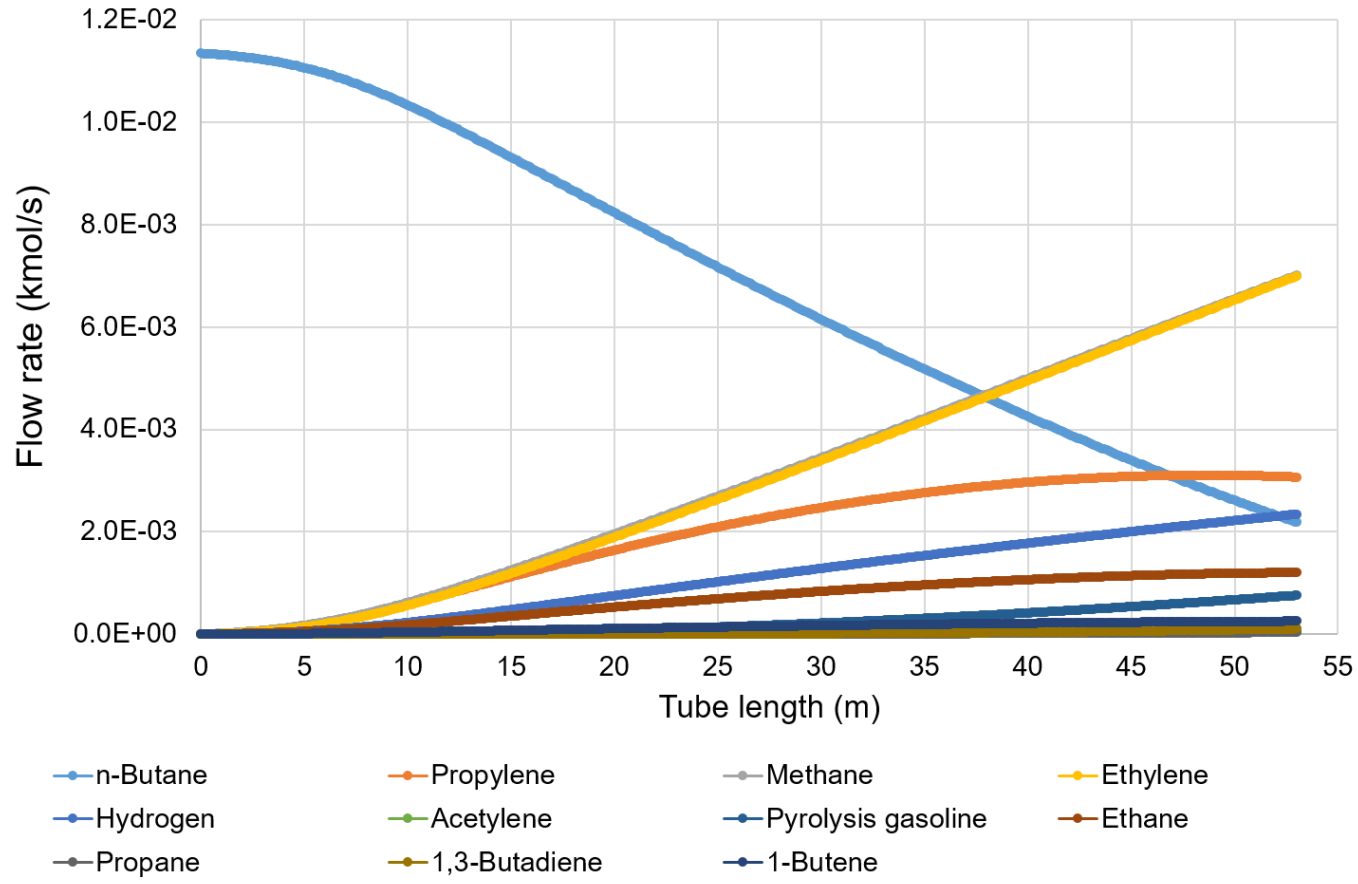

Figure B3. Flow rates of all species along the reactor tube of steam cracking of $n$-butane. 


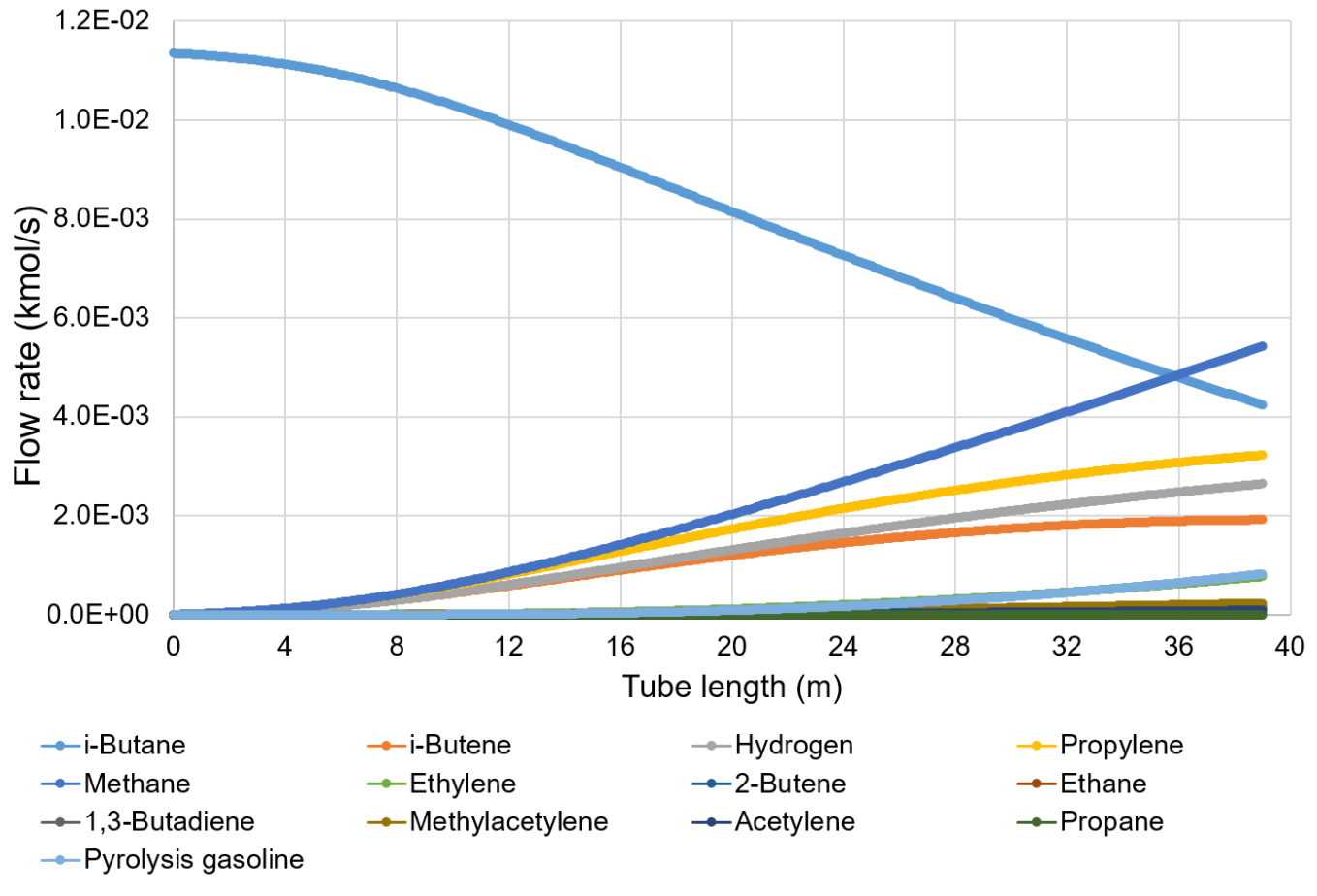

Figure B4. Flow rates of all species along the reactor tube of steam cracking of $i$-butane. 
Table B1. Product distributions of the considered reactions in the hydrocarbons conversion section [283-285, 309]. (unit: wt \%)

\begin{tabular}{|c|c|c|c|c|c|c|c|c|c|c|c|c|}
\hline & \multirow{2}{*}{\multicolumn{2}{|c|}{$\begin{array}{l}\text { C2 Steam } \\
\text { Cracking }\end{array}$}} & \multicolumn{4}{|c|}{ C3 Steam } & \multirow{2}{*}{\multicolumn{2}{|c|}{$\begin{array}{c}n \text {-C4 Steam } \\
\text { Cracking }\end{array}$}} & \\
\hline & & & \multirow{2}{*}{$\begin{array}{c}\text { C2 Oxidative } \\
\text { Dehydrogenation }\end{array}$} & \multicolumn{2}{|c|}{ Cracking } & \multirow{2}{*}{$\begin{array}{c}\mathrm{C} 3 \\
\text { Dehydrogenation }\end{array}$} & & & \multirow{2}{*}{$\begin{array}{c}n-\mathrm{C} 4 \\
\text { Dehydrogenation }\end{array}$} & \multicolumn{2}{|c|}{ Cracking } & \multirow{2}{*}{$\begin{array}{c}i-\mathrm{C} 4 \\
\text { Dehydrogenation }\end{array}$} \\
\hline & $\begin{array}{c}\text { Literatu } \\
\text { re }\end{array}$ & $\begin{array}{c}\text { Optima } \\
1 \\
\end{array}$ & & $\begin{array}{c}\text { Literatu } \\
\text { re }\end{array}$ & $\begin{array}{c}\text { Optima } \\
1 \\
\end{array}$ & & $\begin{array}{c}\text { Literatu } \\
\text { re }\end{array}$ & $\begin{array}{c}\text { Optim } \\
\text { al }\end{array}$ & & $\begin{array}{l}\text { Literat } \\
\text { ure }\end{array}$ & $\begin{array}{c}\text { Optim } \\
\text { al }\end{array}$ & \\
\hline $\mathrm{H}_{2}$ & 4.0 & 3.5 & 1.2 & 1.5 & 1.3 & 4.7 & 1.9 & 0.7 & 1.5 & 1.9 & 0.8 & 1.5 \\
\hline $\mathrm{CH}_{4}$ & 3.8 & 5.1 & 4.5 & 23.4 & 15.9 & 2.8 & 6.6 & 16.4 & 2.5 & 1.8 & 12.5 & 1.0 \\
\hline $\mathrm{C}_{2} \mathrm{H}_{2}$ & 0.4 & 0.4 & 0.1 & 0.5 & 0.2 & 0.0 & 0.0 & 0.2 & 0.0 & 0.0 & 0.3 & 0.0 \\
\hline $\mathrm{C}_{2} \mathrm{H}_{4}$ & 51.9 & 41.1 & 42.9 & 37.2 & 23.7 & 0.2 & 32.9 & 28.6 & 1.0 & 18.8 & 3.1 & 0.0 \\
\hline $\mathrm{C}_{2} \mathrm{H}_{6}$ & 35.0 & 41.5 & 10.6 & 3.1 & 0.5 & 2.2 & 1.5 & 5.3 & 0.6 & 0.4 & 0.6 & 0.2 \\
\hline $\mathrm{C}_{3} \mathrm{H}_{4}$ & 0.0 & 0.0 & 0.0 & 0.5 & 0.0 & 0.0 & 0.0 & 0.0 & 0.0 & 0.0 & 1.3 & 0.0 \\
\hline $\mathrm{C}_{3} \mathrm{H}_{6}$ & 1.2 & 0.7 & 1.6 & 14.8 & 20.1 & 28.0 & 26.3 & 18.8 & 1.0 & 28.2 & 19.5 & 1.5 \\
\hline $\mathrm{C}_{3} \mathrm{H}_{8}$ & 0.1 & 0.7 & 0.2 & 10.0 & 35.2 & 62.0 & 0.1 & 0.2 & 0.0 & 0.0 & 0.0 & 0.0 \\
\hline $\mathrm{C}_{4} \mathrm{H}_{4}$ & 0.1 & 0.0 & 0.0 & 0.1 & 0.0 & 0.0 & 0.0 & 0.0 & 0.0 & 0.0 & 0.0 & 0.0 \\
\hline $\mathrm{C}_{4} \mathrm{H}_{6}$ & 1.8 & 6.9 & 1.5 & 2.9 & 0.8 & 0.0 & 3.8 & 0.8 & 1.0 & 0.9 & 0.1 & 0.2 \\
\hline$n-\mathrm{C}_{4} \mathrm{H}_{8}$ & 0.1 & 0.0 & 0.7 & 0.4 & 0.0 & 0.0 & 17.9 & 2.1 & 39.5 & 0.9 & 0.8 & 4.5 \\
\hline$i-\mathrm{C}_{4} \mathrm{H}_{8}$ & 0.1 & 0.0 & 0.9 & 0.6 & 0.0 & 0.0 & 0.9 & 0.0 & 1.0 & 39.5 & 15.5 & 40.0 \\
\hline$n-\mathrm{C}_{4} \mathrm{H}_{10}$ & 0.1 & 0.0 & 0.0 & 0.0 & 0.0 & 0.0 & 6.0 & 18.5 & 50.0 & 0.9 & 0.0 & 0.9 \\
\hline$i-\mathrm{C}_{4} \mathrm{H}_{10}$ & 0.1 & 0.0 & 0.0 & 0.0 & 0.0 & 0.0 & 0.0 & 0.0 & 1.0 & 6.0 & 35.4 & 50.0 \\
\hline $\mathrm{C} 5+$ & 1.3 & 0.0 & 2.3 & 5.1 & 2.2 & 0.2 & 2.2 & 8.5 & 0.9 & 0.6 & 10.0 & 0.3 \\
\hline $\mathrm{CO}$ & 0.0 & 0.0 & 2.9 & 0.0 & 0.0 & 0.0 & 0.0 & 0.0 & 0.0 & 0.0 & 0.0 & 0.0 \\
\hline $\mathrm{CO}_{2}$ & 0.0 & 0.0 & 4.5 & 0.0 & 0.0 & 0.0 & 0.0 & 0.0 & 0.0 & 0.0 & 0.0 & 0.0 \\
\hline $\mathrm{H}_{2} \mathrm{O}$ & 0.0 & 0.0 & 26.0 & 0.0 & 0.0 & 0.0 & 0.0 & 0.0 & 0.0 & 0.0 & 0.0 & 0.0 \\
\hline
\end{tabular}




\subsection{Appendix C: Model formulation of the superstructure optimization problem}

\subsubsection{Mass balance constraints}

The mass balance relationships in the superstructure are developed following a general mass balance model in previous works [21, 267]. Nine types of flows (“makeup", “up", "reuse”, “in”, “out", “emission”, “down”, "product”, and "recycle” flows) are introduced to describe the mass balance relationships for technology/process $j$ in section $i$. The species are indexed by $k$.

$$
\begin{aligned}
& 0 \leq M_{i, j, k}^{\text {makeup }} \leq Y_{i, j} \cdot u b m u_{i, j, k}, \quad \forall i \in I, j \in J, k \in K \\
& 0 \leq M_{i, j, k}^{\text {reuse }} \leq Y_{i, j} \cdot \text { ubre }_{i, j, k}, \quad \forall i \in I, j \in J, k \in K
\end{aligned}
$$

Constraints (1.C1) and (1.C2) are linking constraints that set the upper and lower bounds of the flow rates of the "makeup" and "reuse" flows. If the technology/process is selected, the corresponding binary variable $Y_{i, j}$ will be equal to 1 and the corresponding flow rates will be allowed to change between the lower and upper bounds. Otherwise, the corresponding binary variable will be 0 and the corresponding flow rates will be forced to be 0 as well.

$$
\sum_{j \in J} M_{l, j, k}^{u p}=w f_{k}^{\text {feed }} \cdot m^{\text {rawshalegas }}, \quad \forall k \in K
$$


There are three steps in the general mass balance model: an inlet converging step, a conversion step, and an outlet separation step. In the first section, the flow rate of each species of the "up" flow is defined using the total feed gas flow rate and its composition as shown Eq. (1.C3).

$$
\sum_{\left(i^{\prime}, j\right) \in U P_{i, l}} M_{i^{\prime}, j, k}^{u p}=\sum_{\left(j, l^{\prime}\right) \in D O W N_{i, l}} M_{i, j, l^{\prime}, k}^{\text {down }}, \quad \forall i \in K, l \in L, k \in K
$$

In Sections 2-8, the flow rates of the "up" flows are equal to the flow rates of the "down" flows in previous sections as shown in Eq. (1.C4). A new index $l$ is introduced to differentiate multiple sub-flows in the same flow category.

$$
\sum_{j \in R E U S E_{i, l}} M_{i, j, k}^{\text {reuse }}=\sum_{\left(i^{\prime}, j, l^{\prime}\right) \in R E C Y C L E_{i, l}} M_{i^{\prime}, j, l^{\prime}, k}^{\text {recyle }}, \quad \forall i \in I, l \in L, k \in K
$$

As shown in Eq. (1.C5), the "reuse" flows sum up a set of "recycle" flows.

$$
M_{i, j, k}^{i n}=M_{i, j, k}^{u p}+M_{i, j, k}^{\text {reuse }}+M_{i, j, k}^{\text {makeup }}, \quad \forall i \in I, j \in J, k \in K
$$

The inlet converging step is modeled by Eq. (1.C6), where the "in" flow of technology/process $j$ in section $i$ combines the "up", the "reuse", and the "makeup" flows that are fed to the same process.

$$
M_{i, j, k}^{i n}=\sum_{k^{\prime} \in K}\left(p_{i, j, k, k^{\prime}}^{i n} \cdot M_{i, j, k^{\prime}}^{i n}\right), \quad \forall i \in I, j \in J, k \in K
$$

The flow rates of various species in the "in" flows are proportional to the key components in the same flows according to the feed composition of the 
corresponding technology/process. The relationships are modeled using parameter $p_{i, j, k, k^{\prime}}^{i n}$ in Eq. (1.C7).

$$
M_{i, j, k}^{\text {out }}=M_{i, j, k}^{i n}+\sum_{k^{\prime} \in K}\left(s c_{i, j, k, k^{\prime}} \cdot M_{i, j, k^{\prime}}^{i n}\right), \quad \forall i \in I, j \in J, k \in K
$$

The conversion step of each technology/process is modeled using Eq. (1.C8), where $s C_{i, j, k, k^{\prime}}$ denotes the product distribution of the chemical reaction.

$$
M_{i, j, k}^{\text {out }}=\sum_{l \in L} M_{i, j, l, k}^{\text {down }}+\sum_{l \in L} M_{i, j, l, k}^{\text {recycle }}+\sum_{l \in L} M_{i, j, l, k}^{\text {product }}+M_{i, j, k}^{\text {waste }}, \quad \forall i \in I, j \in J, k \in K
$$

The "out" flow of a section is split into a set of "down", "recycle", "product", and "waste", flows as shown in Eq. (1.C9).

$$
\begin{aligned}
& M_{i, j, l, k}^{\text {down }}=s f_{i, j, l, k}^{\text {down }} \cdot M_{i, j, k}^{\text {out }}, \quad \forall i \in I, j \in J, l \in L, k \in K \\
& M_{i, j, l, k}^{\text {recycle }}=s f_{i, j, l, k}^{\text {recyle }} \cdot M_{i, j, k}^{\text {out }}, \quad \forall i \in I, j \in J, l \in L, k \in K \\
& M_{i, j, l, k}^{\text {product }}=s f_{i, j, l, k}^{\text {product }} \cdot M_{i, j, k}^{\text {out }}, \quad \forall i \in I, j \in J, l \in L, k \in K
\end{aligned}
$$

Following a common assumption in the literature [38, 310], the mass and energy balance relationships of each technology/process scale linearly. In Eq. (1.C10), the "down" flow comes from the "out" flow based on split fraction $s f_{i, j, l, k}^{\text {down }}$. Similar to the "down" flow, the "recycle" and "product" flows are directly split from the "out" flow as shown in Eqs. (1.C11) and (1.C12). The remaining materials in the "out" flows are automatically treated as the "waste" flow.

$$
M_{k}^{f m}=p^{f m} \cdot \sum_{j \in J, l \in L}\left(M_{2, j, l, k}^{\text {product }}+M_{3, j, l, k}^{\text {product }}\right), \quad \forall k \in K
$$




$$
\begin{aligned}
& M_{i, j, k}^{\text {fuel }}=M_{i, j, k}^{\text {waste }}, \quad \forall i \in I, j \in J, k \in K G \\
& M_{i, j, k}^{\text {wastewater }}=M_{i, j, k}^{\text {waste }}-M_{i, j, k}^{\text {fuel }}, \quad \forall i \in I, j \in J, k \in K \\
& M_{i, j, k}^{\text {fuel }}+\sum_{k^{\prime} \in K}\left(s c_{k, k^{\prime}}^{\text {utility }} \cdot M_{i, j, k^{\prime}}^{\text {fuel }}\right)=M_{i, j, k}^{\text {offgas }}, \quad \forall i \in I, j \in J, k \in K
\end{aligned}
$$

In Eq. (1.C13), a small amount of the pipeline quality gas product is leaked to the atmosphere [291]. The "waste" flows in the superstructure are separated into a fuel gas flow and a wastewater flow as in Eqs. (1.C14) and (1.C15). Finally, Eq. (1.C16) is used to calculate the amount of emissions after the fuel gas is combusted to supply heat and generate electricity onsite.

\subsubsection{Energy balance constraints}

$$
H C_{i, j}=u h c_{i, j} \cdot \sum_{k \in K} M_{i, j, k}^{u p}, \quad \forall i \in I, j \in J
$$

Heating utility consumption is calculated in Eq. (1.C17), where $u_{h} c_{i, j}$ is the unit heating utility consumption rate of technology/process $j$ in section $i$. Four heating utilities are considered, namely low-pressure steam, mid-pressure steam, highpressure steam, and heater.

$$
C C_{i, j}=u c c_{i, j} \cdot \sum_{k \in K} M_{i, j, k}^{u p}, \quad \forall i \in I, j \in J
$$

Cooling utility consumption is calculated in Eq. (1.C18), where ucci,j is the unit cooling utility consumption rate of technology/process $j$ in section $i$. Six cooling 
utilities are considered, namely cooling water, ethane, ethylene, propane, propylene, and Freon 12.

$$
P C_{i, j}=u p c_{i, j} \cdot \sum_{k \in K} M_{i, j, k}^{u p}, \quad \forall i \in I, j \in J
$$

Power consumption is calculated in Eq. (1.C19), where $u p c_{i, j}$ is the unit power consumption rate of technology/process $j$ in section $i$.

$$
P P=\eta^{S T} \cdot\left[\sum_{i \in I, j \in J, k \in K}\left(h h v_{k} \cdot M_{i, j, k}^{\text {fuel }}\right)-\sum_{i \in I, j \in J, k \in K} H C_{i, j, k}^{\text {heater }}\right]
$$

The fuel gas is combusted in the furnace to satisfy a part of the energy requirement in the hydrocarbons conversion section. The off-gas from the furnace is then used to generate electricity via a steam turbine system as modeled in Eq. (1.C20).

\subsubsection{Techno-economic evaluation constraints}

$$
\begin{aligned}
& D C_{i, j}=c c b_{i, j} \cdot\left(\frac{\sum_{k \in K} M_{i, j, k}^{u p}}{m b_{i, j}}\right)^{s c c_{i, j}} \cdot \frac{c e p c i r}{\text { cepcib }_{i, j}}, \quad \forall i \in I, j \in J \\
& D C_{s t}=c c b_{s t} \cdot\left(\frac{P P}{p p b_{s t}}\right)^{s c c_{s t}} \cdot \frac{c e p c i r}{\text { cepcib }_{s t}}
\end{aligned}
$$

Following existing superstructure optimization models $[14,16,310]$, the direct cost of each technology/process is evaluated using power functions as shown in Eq. (1.C21), where $c c b_{i, j}$ is the base-case capital cost, $m b_{i, j}$ is the base-case flow rate,

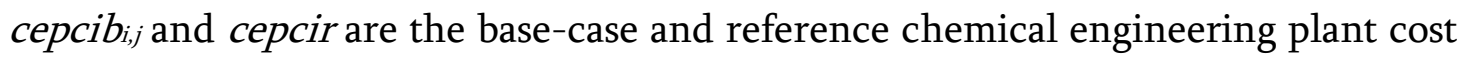


index, respectively. The direct cost of the steam turbine system is evaluated using Eq. (1.C22).

$$
F C I=f c i c \cdot\left(\sum_{i \in I, j \in J} D C_{i, j}+D C_{s t}\right)
$$

The fixed capital investment $F C I$ is evaluated as a percentage of the direct costs as shown in Eq. (1.C23).

$$
W C=w c c \cdot F C I
$$

Based on the fixed capital investment, the working capital $W C$ is defined in Eq. (1.C24).

$$
T C I=F C I+W C
$$

The total capital investment $T C I$ sums up the fixed capital investment and the working capital in Eq. (1.C25).

$$
F C=h \cdot\left[p r_{\text {rawshalegas }} \cdot \sum_{k \in K}\left(e c_{k} \cdot w f_{k}^{\text {feed }} \cdot m^{\text {rawshalegas }}\right)+\sum_{i \in I, j \in J, k \in K}\left(p r_{k} \cdot M_{i, j, k}^{\text {makeup }}\right)\right]
$$

The feedstock cost $F C$ is calculated using Eq. (1.C26), where $h$ is the total hours of operation in a year, $p r_{r a w s h a l e g a s}$ is the price of raw shale gas, $e c_{k}$ is the energy content of species $k$, and $p r_{k}$ is the market price of species $k$.

$$
\begin{aligned}
U C= & h \cdot p r_{\text {heat }} \cdot \sum_{i \in I, j \in J}\left(H C_{i, j}-H C_{i, j, k}^{\text {heater }}\right)+h \cdot p r_{\text {cool }} \cdot \sum_{i \in l, j \in J} C C_{i, j} \\
& +h \cdot p r_{\text {power }} \cdot\left(\sum_{i \in I, j \in J} P C_{i, j}-P P\right)
\end{aligned}
$$


The utility cost $U C$ consists of the costs for purchasing heating utilities, cooling utilities, and electricity as detailed in Eq. (1.C27).

$$
O M C=n o \cdot h s \cdot h \cdot o c c+n s \cdot a s+m c c \cdot\left(\sum_{i \in I, j \in J} D C_{i, j}+D C_{s t}\right)
$$

Eq. (1.C28) defines the operations and maintenance cost $O M C$. no is the number of operators, $h s$ is the operator's salary per hour, occ is the coefficient of operations cost based on the operators' salary, ns is the number of scientists, as is the scientist's salary per year, and mcc is the percentage of the direct costs to account for the maintenance cost.

$$
P T I=p t i c \cdot\left(\sum_{i \in I, j \in J} D C_{i, j}+D C_{s t}\right)
$$

The property tax and insurance $P T I$ is estimated as a percentage of the total direct costs as shown in Eq. (1.C29).

$$
S A_{i, l}=h \cdot p r_{i, l} \cdot \sum_{j \in J, k \in K} m_{i, j, l, k}^{\text {product }}
$$

$S A_{i, l}$ is the sales of product $l$ in section $i$ in Eq. (1.C30).

$$
G E=g e c \cdot \sum_{i \in l, l \in L} S A_{i, l}
$$

The general expense $G E$ is defined by Eq. (1.C31), where gec is the percentage of the general expense of the total sales.

$$
D P C=d p c c \cdot\left(\sum_{i \in I, j \in J} D C_{i, j}+D C_{s t}\right)
$$


For tax purposes, a depreciation cost $D P C$ is calculated using Eq. (1.C32).

$$
I T=\operatorname{tr} \cdot\left(\sum_{i \in l, l \in L} S A_{i, l}-F C-U C-O M C-P T I-G E-D P C\right)
$$

The income tax $I T$ is equal to a percentage of sales less several costs as in Eq. (1.C33).

$$
A C F=\sum_{i \in I, l \in L} S A_{i, l}-F C-U C-O M C-P T I-G E-I T
$$

The annual cash flow $A C F$ account for the sales and the OPEX in Eq. (1.C34).

$$
N P V=\frac{i r \cdot\left(1-i r^{l t}\right)}{1-i r} \cdot A C F-T C I
$$

The net present value $N P V$ is defined in Eq. (1.C35), where ir is the interest rate and $l t$ is the project lifetime.

$$
O B J_{\text {economic }}=\frac{N P V}{l t \cdot h \cdot T E C}
$$

Finally, in Eq. (1.C36), the economic objective function is defined as the NPV per GJ of raw shale gas processed over the entire project lifetime.

\subsubsection{Environmental impact assessment constraints}

$$
E I F=c f_{\text {rawshalegas }} \cdot m^{\text {rawshalegas }}+\sum_{i \in I, j \in J, k \in K}\left(c f_{k}^{\text {makeup }} \cdot M_{i, j, k}^{\text {makeup }}\right)
$$

The environmental impact for raw shale gas extraction and feedstock production $E I F$ is evaluated by Eq. (1.C37), where the $c f$ stands for the characterization factor. 


$$
\begin{aligned}
E I U= & c c f_{\text {heat }} \cdot \sum_{i \in I, j \in J}\left(H C_{i, j}-H C_{i, j, k}^{\text {heater }}\right)+c f_{\text {cool }} \cdot \sum_{i \in I, j \in J} C C_{i, j} \\
& +c f_{\text {power }} \cdot\left(\sum_{i \in I, j \in J} P C_{i, j}-P P\right) \\
E I W= & c f_{w w t} \cdot \sum_{i \in I, j \in J, k \in K} M_{i, j, k}^{\text {wastewater }} \\
E I D= & \sum_{k \in K}\left(c f_{k} \cdot M_{k}^{f m}\right)+\sum_{i \in I, j \in J, k \in K}\left(c f_{k} \cdot M_{i, j, k}^{\text {offgas }}\right)
\end{aligned}
$$

The environmental impacts associated with utility production EIU, wastewater treatment $E I W$, and direct emissions EID are defined by Eqs. (1.C38)-(1.C40), respectively.

$$
\begin{aligned}
& O B J_{G W P}=\frac{(E I F+E I U+E I W+E I D)_{G W P}}{T E C} \\
& O B J_{\text {water }}=\frac{(E I F+E I U+E I W)_{\text {water }}}{T E C}
\end{aligned}
$$

One of the environmental objective functions is defined in Eq. (1.C41) to minimize the unit GWP. In the first case study, only the shale gas processing part of the superstructure is considered and the objective function is to minimize the GWP per 1 GJ of shale gas processing products. Therefore, the numerator of the fractional term of Eq. (1.C41) accounts for the direct and indirect emissions associated with the shale gas processing part of the superstructure, while the denominator TEC is defined as $\sum_{i \in I P, j \in J, l \in L, k \in K}\left(e c_{k} \cdot M_{i, j, l, k}^{\text {product }}\right)$. In the other case studies, the entire superstructure is considered and the objective function is defined as the 
GWP per $1 \mathrm{GJ}$ of raw shale gas. The numerator of the fractional term of Eq. (1.C41) accounts for the direct and indirect emissions associated with the entire integrated shale gas processing and chemical manufacturing process and the corresponding $T E C$ is defined as $\sum_{k \in K}\left(e c_{k} \cdot w f_{k}^{f e e d} \cdot m^{\text {rawshalegas }}\right)$. The other environmental objective function in Eq. (1.C42) minimizes the unit water footprint. The total water footprint sums up the direct water consumption in the integrated shale gas processing and chemical manufacturing process, indirect water consumption in feedstock extraction and utility consumption, and indirect water production in wastewater treatment.

\subsubsection{Superstructure network configuration constraints}

$$
\begin{aligned}
& \sum_{j \in J} Y_{i, j}=1, \quad \forall i \in\{1,2,3,4,8\} \\
& Y_{2,1}=Y_{3,1} \\
& Y_{5,1}+Y_{5,2}=1 \\
& Y_{5,3}+Y_{5,4}=1 \\
& Y_{5,5}+Y_{5,6}=1 \\
& Y_{5,7}+Y_{5,8}=1 \\
& Y_{5,1}=Y_{6,1}+Y_{6,2}+Y_{6,3} \\
& Y_{5,2}=Y_{6,4} \\
& Y_{5,3}=Y_{6,5}+Y_{6,6}+Y_{6,7}
\end{aligned}
$$




$$
\begin{aligned}
& Y_{5,4}=Y_{6,8}+Y_{6,9}+Y_{6,10} \\
& Y_{5,5}=Y_{6,11}+Y_{6,12}+Y_{6,13} \\
& Y_{5,6}=Y_{6,14}+Y_{6,15}+Y_{6,16} \\
& Y_{5,7}=Y_{6,17}+Y_{6,18}+Y_{6,19} \\
& Y_{5,8}=Y_{6,20}+Y_{6,21}
\end{aligned}
$$

To describe the network configuration, the sections of acid gas removal, dehydration, NGLs recovery, NGLs separation, hydrocarbons conversion, light olefins separation, C4 separation, and acid gas disposal are indexed by $i=1-8$, respectively. Furthermore, the first technology/process in each section is indexed by $j=1$ and index of any other technology/process is equal to the index of the technology/process above it plus 1 . Consequently, each technology/process is assigned with a unique pair of indices $(i, j)$. Then, binary variable $Y_{i, j}$ is introduced for each technology/process. When $Y_{i, j}$ is equal to 1 , the corresponding technology/process is selected, and 0 otherwise $[14,16,19,307]$. Eq. (1.C43) requires that one and only one technology/process is selected in the sections of acid gas removal, dehydration, NGLs recovery, NGLs separation, and acid gas disposal. Since the condensation process in the dehydration section is integrated with a tailored NGLs recovery, they are selected or rejected simultaneously as enforced by Eq. (1.C44). In Eqs. (1.C45)-(1.C48), only one from the two technologies is selected when upgrading the hydrocarbons. In Eqs. (1.C49)-(1.C56) 
, one from the three separation technologies is selected for each product from the hydrocarbons conversion section.

\subsection{Appendix D: Model formulation of the auxiliary parametric}

\section{problem}

$$
\begin{aligned}
& D C_{i, j}=\sum_{p \in P}\left(a c_{i, j, p} \cdot X C_{i, j, p}+b c_{i, j, p} \cdot Y C_{i, j, p}\right), \quad \forall i \in I, j \in J \\
& \sum_{p \in P} Y C_{i, j, p}=1, \quad \forall i \in I, j \in J \\
& \sum_{p \in P} X C_{i, j, p}=\sum_{k \in K} M_{i, j, k}^{u p}, \quad \forall i \in I, j \in J \\
& m c_{i, j, p-1} \cdot Y C_{i, j, p} \leq X C_{i, j, p} \leq m c_{i, j, p} \cdot Y C_{i, j, p}, \quad \forall i \in I, j \in J, p \in P \\
& \quad \frac{c c b_{i, j}}{\left(m b_{i, j}\right)^{s c f_{i, j}} \cdot \frac{c e p c i r}{c e p c i b_{i, j}} \cdot\left[\left(m c_{i, j, p}\right)^{s c f_{i, j}}-\left(m c_{i, j, p-1}\right)^{s c c_{i, j}}\right]} \quad m c_{i, j, p}-m c_{i, j, p-1} \\
& b c_{i, j, p}=c c b_{i, j} \cdot\left(\frac{m c_{i, j, p}}{m b_{i, j}}\right)^{s c f_{i, j}} \cdot \frac{c e p c i r}{c e p c i b_{i, j}}-a c_{i, j, p} \cdot m c_{i, j, p}, \quad \forall i, j, p \\
& D C_{s t}=\sum_{p \in P}\left(a s t_{p} \cdot X S T_{p}+b s t_{p} \cdot Y S T_{p}\right) \\
& \sum_{p \in P} Y S T_{p}=1 \\
& \sum_{p \in P} X S T_{p}=P P \\
& m s t_{p-1} \cdot Y S T_{p} \leq X S T_{p} \leq m s t_{p} \cdot Y S T_{p}, \\
& \forall p \in P
\end{aligned}
$$




$$
\begin{gathered}
a s t_{p}=\frac{\frac{c c b_{s t}}{(p p b)^{s f_{s t}} \cdot \frac{c e p c i r}{c e p c i b_{s t}} \cdot\left[\left(m s t_{p}\right)^{s c c_{s t}}-\left(m s t_{p-1}\right)^{s c c_{s t}}\right]}}{m s t_{p}-m s t_{p-1}}, \quad \forall p \in P \\
b s t_{p}=c c b_{s t} \cdot\left(\frac{m s t_{p}}{p p b}\right)^{s c f_{s t}} \cdot \frac{c e p c i r}{c e p c i b_{s t}}-a s t_{p} \cdot m s t_{p}, \quad \forall p \in P
\end{gathered}
$$

The separable concave terms in Eqs. (1.C21) and (1.C22) can be approximated by piecewise linear approximation functions (1.D1)-(1.D12). $P$ represents a set of partition points; continuous variables $X C_{i, j p}$ and $X S T$ are introduced to connect with the original input variables of the nonlinear functions in Eqs. (1.D3) and (1.D9); integer variables $Y C_{i, j p}$ and $Y S T$ are introduced to select a piece of the piecewise linear functions.

$$
\begin{aligned}
& (E I F+E I U+E I W+E I D)_{G W P} \leq T E C \cdot \varepsilon l \\
& (E I F+E I U+E I W)_{\text {water }} \leq T E C \cdot \varepsilon 2 \\
& O B J_{\text {algorithm }}=N P V-r \cdot h \cdot l s \cdot T E C
\end{aligned}
$$

Based on the piecewise linear approximation functions, we introduce an auxiliary parameter $r$ and an auxiliary parametric problem with respect to $r$. The general model formulation of this parametric objective function is shown below.

$\max \quad O B J_{\text {algorithm }}$ in (1.D15)

s.t. original constraints (1.C1)-(1.C20), (1.C23)-(1.C35), (1.C37)-(1.C40), (1.C43) $-(1 . \mathrm{C} 56)$

piecewise linear approximation functions (1.D1)-(1.D12) additional constraints for environmental objective functions (1.D13)-(1.D15) 
CHAPTER 6

RESILIENT DESIGN AND OPERATIONS OF ENERGY SYSTEMS

\subsection{Introduction}

In this chapter, we address the resilient design and operations of process systems in response to disruption events. An improved quantitative measure of resilience is proposed as the ratio of the quantity of products manufactured with disruptive events to that without disruptive events. Five resilience enhancement strategies are then introduced, including selecting the most resilient technology/process alternatives, increasing the capacities of operating processes, employing parallel operating processes, building backup processes, and optimizing the operating levels after the occurrence of disruptive events. A general framework for resilience optimization is further proposed to incorporate the quantitative measure of resilience and the resilience enhancement strategies into process design and operations. In the first step of the proposed resilience optimization framework, a preliminary risk assessment is performed for a given system to identify the disruptive events that are worth considering in process design and operations. The numbers of failed processes for the identified disruptive events and the recovery time of each process are used as input parameters for resilient design and operations. In the second step, a multiobjective two-stage adaptive robust mixed- 
integer fractional programming (ARMIFP) model is formulated. There are two objective functions: the first objective function is to maximize the resilience under the worst-case realization of disruptive events, and the second objective function is to minimize the total capital cost. Both objective functions are independent of external processes and volatile markets, thus reflecting the intrinsic properties of the given system. As external processes and volatile markets would influence the operating expenditures (OPEX) after the occurrence of disruptive events, OPEX is not included in the economic objective function. The resulting optimization model has a three-level structure: the first level determines the optimal network configuration, equipment capacities, and capital costs; the second level determines the worst-case realization of disruptive events; the third level determines the optimal number of available processes and operating levels in each time period. To tackle the computational challenges stemming from the multilevel structure and the nonlinear objective function, a tailored global optimization method is proposed by integrating the inexact parametric algorithm and the column-and-constraint generation algorithm. The applicability of the proposed resilience optimization framework is illustrated through two applications on process design and planning of a chemical process network and superstructure optimization of shale gas 
processing and natural gas liquids (NGL) recovery processes, respectively. The novelties of this work are summarized below:

- A novel multiobjective ARMIFP model for resilient design and operations;

- A tailored solution algorithm for efficient global optimization of the resulting ARMIFP problems;

- Novel applications to resilient design and planning of a chemical process network and superstructure optimization of shale gas processing and NGLs recovery processes.

The rest of this paper is organized as follows. The quantitative measure of resilience and a set of resilience enhancement strategies are described in the next two sections, followed by the Problem Statement. We then present the general framework for resilience optimization. Two applications of the proposed resilience optimization framework are presented in the following sections. Conclusion is provided at the end.

\subsection{Quantitative measure of resilience}

Common disruptive events include natural disasters (such as tornados, earthquakes, and hurricanes), process accidents (such as faulty operations), and intentional man-made attacks (such as terrorism and sabotage) [154]. Many system performance indicators, such as the flow rates of key streams and the number of normally functioning processes, respond to disruptive events dynamically and reflect the impacts of disruptive events to a given system. Figure 59 illustrates the 
three sequential phases of the performance of a system performance function $F(t)$. The disruptive events occur at to and cause initial failures in the process system. The first phase, or the impact propagation phase, lasts from to to $t$. During this period, the initial failures cause additional failures in other normally functioning processes. Therefore, the system performance function keeps declining until $t$, which corresponds to the lowest operating level. In the system recovery phase from $t_{1}$ to $t_{2}$, the failed processes in the given system recovers gradually and the system performance function increases to its original operating level. In the last phase from $t_{2}$ to $t_{n}$, the system maintains the operating level. 


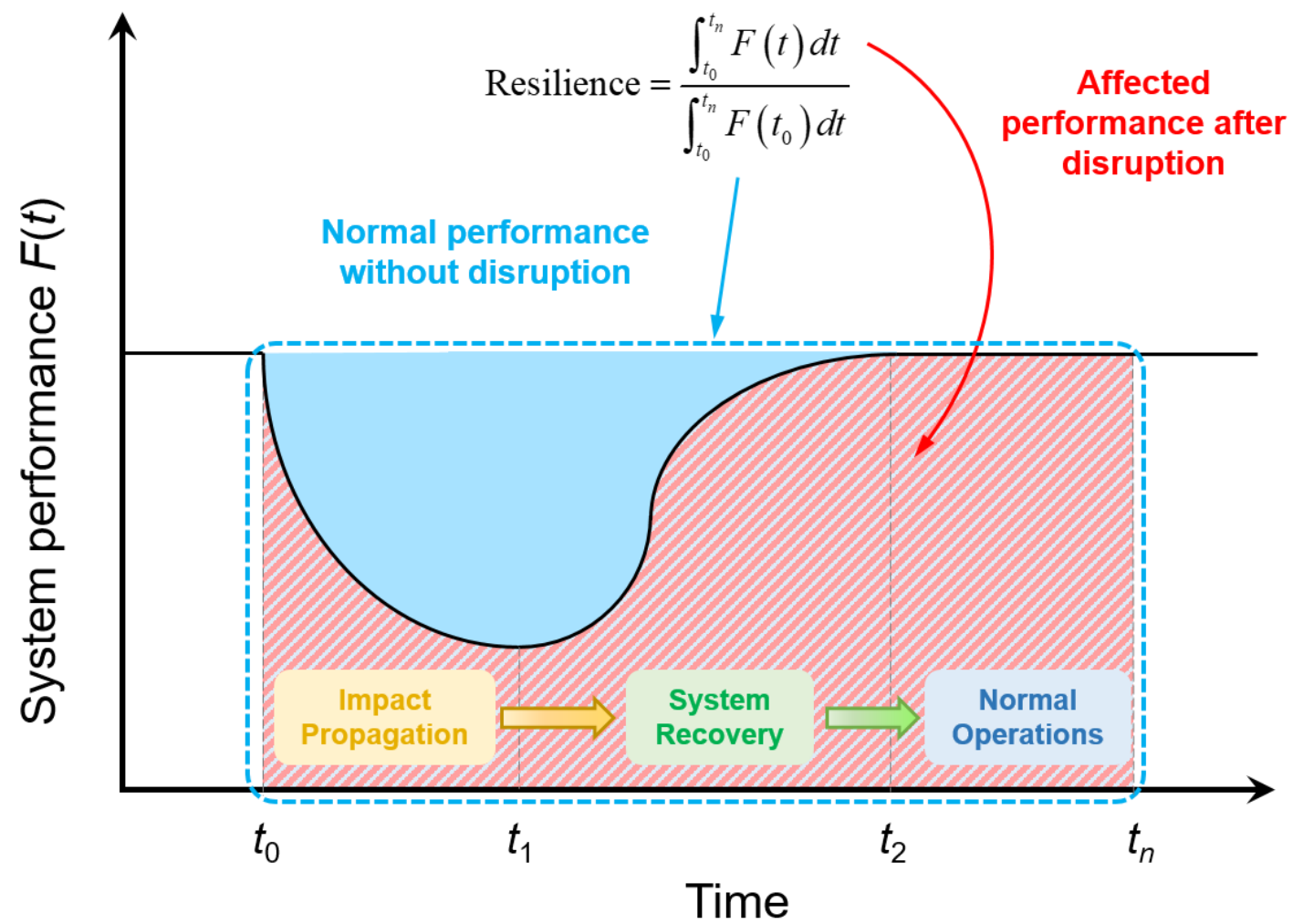

Figure 59. Definition of resilience.

There are several ways to measure the impacts of disruptive events and quantify the resilience. One way is to measure the recovery time, or $t_{2}-t$, which indicates how long the given system can regain the original operating level [120]. Another way is to measure the performance degradation, or $F\left(t_{1}\right)-F\left(t_{0}\right)$, which calculates the maximum decline in the operating level after the disruptive events [120]. Alternatively, resilience was calculated as the mean ratio of absolute quantities $[311,312]$, the mean ratio of areas under performance curves [121], the ratio of durations [313], and the ratio of recovery to loss at each time point [314]. 
Resilience was also defined indirectly as the probability given the maximum acceptable performance loss and recovery time [315], the conditional probability of a satisfactory state [316], the sum of factors for system safety [317], and the weighted sum of resilience costs [318]. Among these definitions, ratio measures are straightforward to understand and to compare with different systems. However, the existing ratio measures are not suitable for process systems and can cause computation difficulties. In the definition by Bruneau et al. [128], the maximum operating level of the performance function was known and set as 100 . Nevertheless, this maximum operating level in a process design and operations problem is unknown before the optimization problem is solved. Therefore, the maximum operating level should be treated as a variable. In another definition by Ouyang and Duenas-Osorio [319], resilience was measured as a time-dependent ratio of the accumulated performance with disruptive events to that without disruptive events. Since this measure was proposed for infrastructure systems with continuous performance functions, the resulting optimization model consists of integral terms in both numerator and denominator of the fractional objective function. As a result, global optimization of the resulting dynamic optimization problem would be quite challenging. An improved quantitative measure of resilience is proposed in this chapter to address the above issues. 


$$
\text { Resilience }=\frac{\int_{t_{0}}^{t_{n}} F(t) d t}{\int_{t_{0}}^{t_{n}} F\left(t_{0}\right) d t}
$$

The denominator of Eq. (1.16) calculates the accumulated system performance from to to $t_{\mathrm{n}}$ and the numerator of Eq. (1.16) calculates the accumulated system performance in the same period but assumes that no disruptive event occurs. A key advantage of this quantitative measure is that it accounts for recovery time and performance degradation simultaneously. Moreover, as the quantitative measure is normalized by the accumulated system performance without disruptions, the resilience analysis results can be compared among distinct process systems. The failed processes of a given system can be reused only if they are fully repaired and tested. Therefore, system performance remains the same in each time period, and the system performance functions are step-wise functions. Based on this property, the integral terms in Eq. (1.16) are equal to the corresponding summation terms in Eq. (1.17), where $t i$ is the length of each time period. The time horizon is divided into a set of time periods. The performance function stays unchanged when a process is under recovery, and the performance function increases at the end of a time period when recovery completes. Therefore, the step-wise performance function for process systems can be naturally discretized. 


$$
\begin{aligned}
& \text { Resilience }=\frac{\sum_{w=0}^{\frac{t_{n}-t_{0}}{t i}-1}\left[F\left(t_{0}+w \cdot t i\right) \cdot t i\right]}{F\left(t_{0}\right) \cdot\left(t_{n}-t_{0}\right)} \\
& F(t)=\sum_{k}\left[\omega_{k} \cdot M(t)_{k}\right]
\end{aligned}
$$

An important component of the proposed measure in Eq. (1.17) is the system performance function $F(t)$. Since resilience is an intrinsic property of the system, a system performance indicator should be independent of external processes or volatile markets, and should respond to the disruptive events dynamically. In this chapter, the system performance function is defined in Eq. (1.18), where $M(t)_{k}$ and $\omega_{k}$ are the flow rate and the weight of product $k$, respectively. The weights can be defined by decision makers, or using the intrinsic properties of products, such as energy contents.

The proposed quantitative measure of resilience is different from those in the existing literature. In the definition by Bruneau et al. [128], the maximum operating level of the performance function was known and set as 100 . However, this maximum operating level in a process design and operations problem is unknown before the optimization problem is solved. Therefore, the maximum operating level is treated as a variable in the denominator of the proposed resilience measure. In another relevant definition by Ouyang and Duenas-Osorio [319], resilience was measured as a time-dependent ratio of the accumulated 
performance with disruptive events to that without disruptive events. There are several differences between the proposed measure and the one in the literature. First, the proposed measure considers the same time horizon for different process designs in order to make a fair comparison, while the one in the literature is timedependent. Second, the operating level without disruptive events in the proposed resilience measure is equal to the operating level before the occurrence of disruptive events, while the literature one is modeled as a function of time. Third, the product flow rates of a process system are step-wise functions and the measure in Eq. (1.16) can be reformulated to the one in Eq. (1.17). Given that the measure in the literature is used for infrastructure systems with continuous performance functions, a similar reformulation would not be rigorous. Therefore, the proposed resilience is more suitable for process systems than those reported in the literature.

\subsection{Resilience enhancement strategies}

In the existing studies for resilience, many strategies have been developed to mitigate the impacts of disruptive events $[121,136,146]$. For example, the average time of a customer with no power access could be reduced effectively if the optimal post-earthquake recovery schedule of a power system was employed [136]. However, only a few resilience enhancement strategies have been considered in each study and a summary of resilience enhancement strategies is still not available 
in the literature. In this chapter, we introduce five resilience enhancement strategies for process systems. The first four strategies are related to process design and can be implemented before the disruptive events occur, while the last strategy is related to process operations and can be implemented only after the disruptive events occur. The details of the resilience enhancement strategies are provided as follows.

- The first strategy is to select the most resilient process design from a set of alternative process designs. This strategy corresponds to determining the optimal design decisions of a network system [320]. If several technology/process alternatives can complete the same task in a process system, the one that demonstrates the shortest time to recover from damages caused by disruptive events would be preferred. A combination of different processes for manufacturing the same intermediate or final product can also reduce the impacts of disruptive events.

- The second strategy is to increase the capacities of the selected operating processes beyond their nominal operating levels. This strategy was also referred to as the absorptive capacity in infrastructure network problems [146]. In the case where several different processes can manufacture the same intermediate or final product, the increased capacity of one process can make up the loss of another one's.

- The third strategy is to employ multiple parallel operating units in place of a single operating unit with the same total capacity. This strategy was highlighted in the 
optimization models for reliable process designs $[152,153]$. Process units with smaller capacities tend to recover (including shutting down, repair, and starting up) in a shorter time than the corresponding ones with larger capacities. Moreover, once one of the failed parallel operating units is recovered, the system can resume the production instantly.

- The fourth strategy is to build backup processes in addition to operating processes. This strategy was also referred to as redundancy in the literature [128]. Unlike operating processes, backup processes are not used during normal operations. A major advantage of backup processes is that a portion of or even the entire capacity can be instantly recovered after the occurrence of disruptive events.

- The fifth strategy is to optimize the operating levels after the occurrence of disruptive events. This strategy corresponds to determining the optimal recovery scheduling decisions and the optimal operational decisions after the occurrence of disruptive events [136]. During system recovery, the available capacity of each process keeps changing. To maximize resilience, it is important to determine the maximum operating levels of each process and the maximum product rate in each time period.

The aforementioned strategies aim to enhance the resilience of a process system, but they come at a cost that may overwhelm the enhancement in resilience. In practice, there is typically a trade-off between capital investment and systems resilience. Therefore, it is necessary to address the resilience and the total capital 
cost simultaneously, which motivates the use of a bi-criterion optimization approach in the proposed resilience optimization framework.

\subsection{Problem statement for resilience optimization}

In this chapter, we address the resilient design and operations of a process system in response to disruptive events, based on the proposed quantitative measure of resilience and five resilience enhancement strategies. We are given a process system with many interconnected processes. Alternatively, we are given a superstructure that incorporates a number of process design alternatives. A set of disruptive events may impact part of or even the entire system. The following parameters are also known:

- The physical properties of the involved species;

- The upper and lower bounds of the capacity of each technology/process alternative;

- The base-case capital costs, flow rates, scaling factors, and chemical engineering plant cost indices of all the processes;

- The recovery time of each process;

- The composition and the upper bound of the feedstock flow rate;

- The composition of the feed to each process;

- The product distributions of the involved reactions;

- The split fractions of each species to various flows;

- The demand of various products; 
The problem is formulated in discrete time with a finite time horizon. The occurrence of disruptive events gives rise to failures in a set of process units at the beginning of the first time period. After a disruptive event occurs, a process influenced by the disruptive event can be denoted as either failed or functioning normally. However, the availability of each process is unknown until the system is thoroughly inspected and tested. Therefore, the availability of each process after the occurrence of disruptive events is considered as uncertain, which can be quantitatively described using an uncertainty set. Two additional parameters are introduced in the uncertainty set to enforce an upper bound of the total availability of the processes: (1) The numbers of failed processes after the occurrence of disruptive events, which can be estimated from historical records to reflect the impacts of the disruptive events; (2) A tolerance level, which is introduced to control the conservatism of the robust optimal solution.

A resilient design of the process system may correspond to a significantly high capital cost, which is undesirable from an economic perspective. Therefore, the goal of this problem is to determine the optimal process design by maximizing the resilience under the worst-case realization of disruptive events and minimizing the total capital cost simultaneously. OPEX is not considered in the economic objective function for two reasons: (1) As the operating levels keep changing after the 
occurrence of disruptive events, a low OPEX after the occurrence of disruptive events does not necessarily suggest a low OPEX with normal operating conditions; (2) Given that the resilience objective function reflects an intrinsic property of the process system, the economic objective function excludes OPEX to avoid the influence from external processes and volatile markets.

In the two-stage adaptive robust optimization problem, decisions are made sequentially in two stages: (1) In the first stage, the "here-and-now" decisions are made before disruptive events occur; (2) In the second stage, the "wait-and-see" decisions are made after the failed processes in the given system are revealed. The first-stage decision variables include:

- Technology/process selection in the optimal process design;

- The capacities of the operating processes and backup processes;

- The capital costs of the operating processes and backup processes.

The second-stage decision variables include:

- The number of available operating processes and backup processes in each time period after the occurrence of disruptive events;

- The operating levels of various flows in each process in each time period.

\subsection{General framework for resilience optimization}

To mitigate the impacts of disruptive events, it is critical to account for resilience systematically in process design and operations. We propose a general framework 
for resilience optimization that incorporates the quantitative measure of resilience and the five resilience enhancement strategies. As shown in Figure 60, there are three steps in the proposed resilience optimization framework. In the first step, a preliminary risk assessment is performed for a given system. A set of disruptive events are identified, and the related information, such as the number of failed processes in the given system and the recovery time of each process, is used as input parameters in the second step to formulate a multiobjective two-stage ARMIFP model. The model includes two objective functions: maximizing the resilience under the worst-case realization of disruptive events and minimizing the total capital cost. Moreover, the model consists of three levels: the first level determines the optimal network configuration, equipment capacities, and capital costs; the second level determines the worst-case realization of disruptive events; the third level determines the optimal number of available processes and operating levels in each time period. 


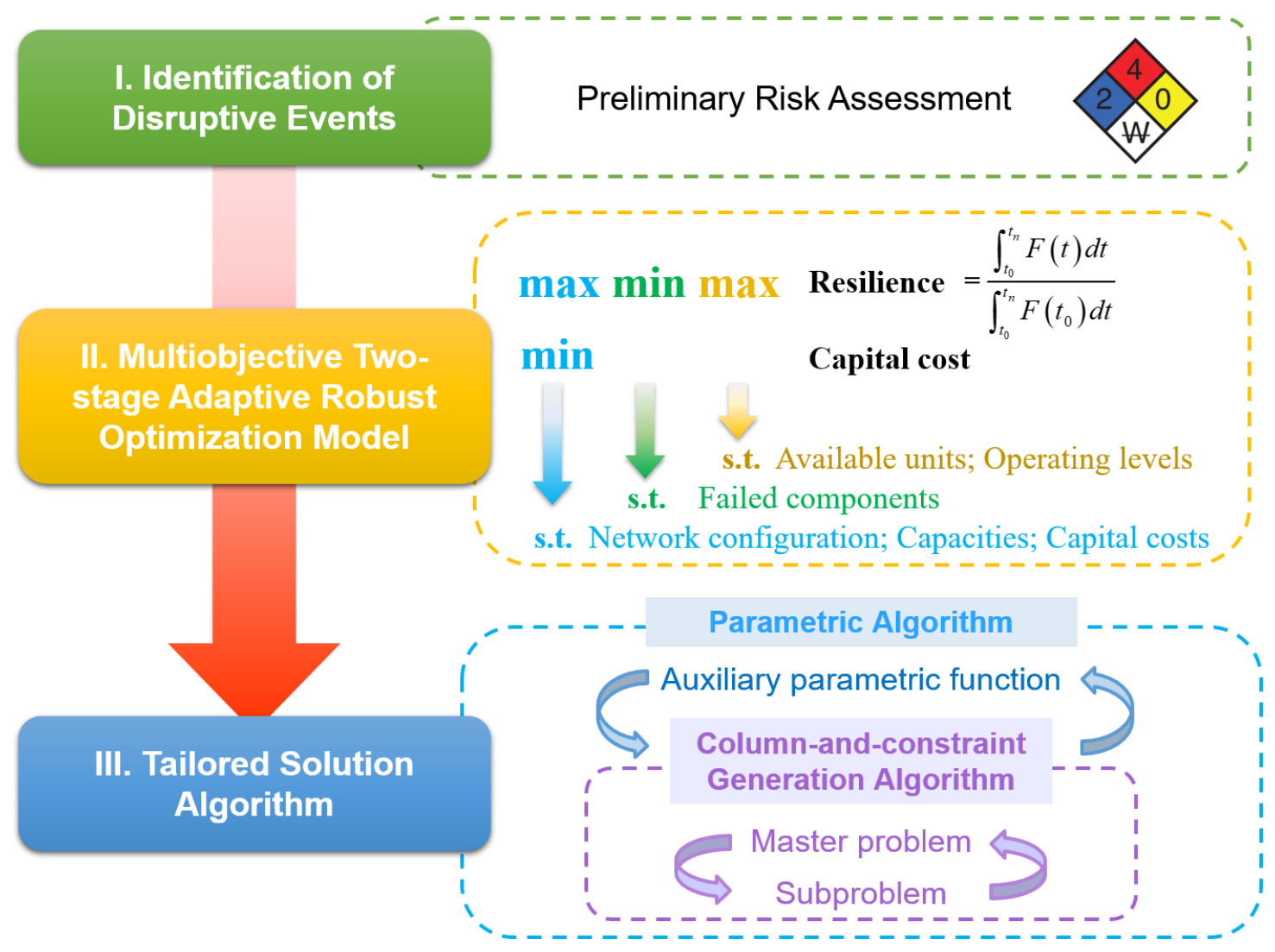

Figure 60. General framework of resilience optimization.

In the third step of the proposed resilience optimization framework, the multiobjective two-stage ARMIFP problem is solved by a tailored global optimization method that integrates the parametric algorithm and the columnand-constraint generation algorithm. The details of the proposed resilience optimization framework are presented in the following subsections.

\subsubsection{Identification of disruptive events}

The first step of the proposed resilience optimization framework is to identify the disruptive events that should be considered in the resilient design and operations. 
For a given system, there can be a number of potential disruptive events with varying probabilities of occurrence and varying levels of severity. The disruptive events that are more frequent and severe tend to be more destructive, while others may not deserve much attention in the design and operations. Therefore, there is a need of conducting a preliminary risk assessment to screen the potential disruptive events and identify the destructive ones that are worth considering in process design and operations. This preliminary risk assessment can be conducted using a risk matrix method [321]. This method characterizes a disruptive event with a probability score and a severity score. The combinations of the probability score and the severity score are then translated to multiple risk levels and displayed in a matrix. As a result of the preliminary risk assessment, priorities should be given to high-level and moderate-level disruptive events.

An example of the risk matrix method is given as follows. First, five probability intervals are matched with 5 probability scores: a probability lying in $[0,20 \%)$, $[20 \%, 40 \%),[40 \%, 60 \%),[60 \%, 80 \%)$, and $[80 \%, 100 \%]$ corresponds to a probability score of 1, 2, 3, 4, and 5, respectively. Second, five cost intervals are matched with 5 severity scores: an increase in the production cost within $[0,1 \%)$, $[1 \%, 5 \%),[5 \%, 10 \%),[10 \%, 20 \%)$, and $[20 \%,+\infty)$ corresponds to a severity score of $1,2,3,4$, and 5 , respectively. Next, three risk levels are determined based on the 
scores: (1) A low-risk level if the probability score is equal to 1 , or if the probability score is greater than 1 and the sum of the probability score and the severity score is smaller than or equal to 5; (2) A moderate-risk level if the probability score is greater than 1 and the sum of the probability score and the severity score is greater than 5 but smaller than 8; (3) A high-risk level if the sum of the probability score and the severity score is greater than or equal to 8 . Finally, the disruptive events with a moderate-risk level or a high-risk level are considered in the next step. Several key parameters of the identified disruptive events, such as the number of failed processes in the given system and the recovery time of each process, are used as input parameters of the optimization model for resilient design and operations.

\subsubsection{Multiobjective two-stage adaptive robust optimization model}

Given the identified disruptive events from the first step, a multiobjective twostage ARMIFP model is developed for resilient design and operations. The decisions are determined sequentially in two stages. The first-stage decisions, including network configuration, equipment capacities, and capital costs, are determined before the occurrence of the disruptive events; the second-stage decisions, including the number of available processes and operating levels in each time period, are determined after the occurrence of the disruptive events. 


$$
\begin{aligned}
& \max \min _{S I \in U} \max _{(Z, Z R, M) \in O} \frac{\sum_{i \in I, j \in J, k \in K, t \in T} M_{i, j, k, t}^{\text {product }}}{\sum_{i \in I, j \in J, m \in M, k \in K}\left(n u_{m} \cdot C A_{i, j, m, k}^{\text {product }}\right) \cdot t t} \\
& \min \sum_{i \in I, j \in J}\left[\sum_{m \in M}\left(n u_{m} \cdot C C_{i, j, m}\right)+C C R_{i, j}\right]
\end{aligned}
$$

s.t. network configuration constraints $(1.21)-(1.22)$

equipment capacity constraints $(1.23)-(1.25)$

capital cost evaluation constraints $(1.26)-(1.41)$

Where $U=\left\{S I \in\{0,1\}^{|I| \times|D|}:\right.$ constraints (4) $\left.-(5)\right\}$,

$O=\{$ recovery constraints $(27)-(32)$; operating level constraints $(33)-(47)\}$.

In robust optimization, the worst-case uncertainty realization should be determined before making the decisions that are affected by uncertainty. In this proposed ARMIFP model, the availability of process units is uncertain after the occurrence of disruptive events. Therefore, a minimization problem must be solved to determine the worst-case realization of the availability of process units before the second-stage decisions are made. Following the existing two-stage adaptive robust optimization models $[56,155,322,323]$, the proposed ARMIFP model has a three-level structure: the first-level optimization problem determines the optimal first-stage decisions, including network configuration, equipment capacities, and capital costs; the second-level optimization problem determines the worst-case realization of the availability of process units; the third-level optimization problem determines the optimal second-stage decisions, including the number of available processes and operating levels in each time period. Unlike the existing models 
$[155,156,227]$, the proposed model includes two objectives. The first objective is to maximize the resilience under the worst-case realization of disruptive events, and the second objective is to minimize the total capital cost of the given system. In the proposed model, indices $i, j, k, m$, and $v$ are for sections, technology and process alternatives, parallel operating processes, species, and network connection constraints, respectively. A list of indices/sets, parameters and variables is given in the Nomenclature, where all parameters are denoted in lower-case symbols or Greek letters, and all variables are denoted with a capitalized first letter. The details of the constraints are presented in the following subsections.

\subsubsection{Uncertainty set}

Depending on many external and internal factors, a disruptive event can impact

only one process or multiple processes in the given system. External factors include, but not limit to, the type, scale, and severity of the disruptive event, the start time of the disruptive event, the duration of the disruptive event, and the environment of the given system. Internal factors include, but not limit to, the use of advanced sensors, monitors, and process control technologies, the precautions against the disruptive event, and the contingency plans for the disruptive event. However, due to the uncertain nature of disruptive events, it is impossible to 
predict precisely whether a process will be affected by a specific disruptive event or not.

$$
\begin{aligned}
& \sum_{i \in I D_{d}} S I_{i, d} \leq \Gamma_{d} \cdot\left(1-\varepsilon_{d}\right), \quad \forall d \in D \\
& \sum_{i \notin D_{d}} S I_{i, d}=0, \quad \forall d \in D
\end{aligned}
$$

In the proposed multiobjective two-stage ARMIFP model, the uncertain availability of section $i$ after the occurrence of disruptive event $d$ is modeled as a binary variable $S I_{i, d .} S I_{i, d}$ is equal to one if the operating processes in section $i$ fail after the occurrence of disruptive event $d$, and 0 otherwise. Given the same operating conditions, parallel operating processes are assumed to show the same availability after the occurrence of disruptive event $d$. Additionally, constraints (1.19) and (1.20) are introduced to provide an upper bound of the number of failed processes in the given system. $\Gamma_{d}$ denotes the number of failed processes of disruptive event $d$ based on historical records or simulation results. As a decisionmaker may consider it too conservative to hedge against the realization where $\Gamma_{d}$ processes fail, a tolerance level $\varepsilon_{d}$ is introduced to adjust the degree of conservatism of the optimal solution. Since a disruptive event may influence only a set of processes, the summation in constraint (1.19) are limited to a subset of sections $I D_{d .}$ The processes not in subset $I D_{d}$ are fixed to be 0 as enforced by constraint (1.20). 
In the traditional robust optimization approach, the total deviation of uncertain parameters from their nominal values are not restricted, so the corresponding variables in the uncertainty set can take any value in their intervals. Therefore, it is unnecessary to include a second-level optimization problem in the model, because the worst-case realization can be easily predetermined by taking the worst-case value of each variable. In the proposed adaptive robust optimization problem, it is practically unnecessary to build resilient processes to hedge against all possible disruptive events. Similar to budge of uncertainty [155], we constrain the total deviation of uncertainty by introducing an acceptable total number of failed processes, which is the product of a historical total number of failed processes $\Gamma_{d}$ and decision makers' tolerance level $\varepsilon_{d}$. Therefore, it becomes nontrivial to determine the worst-case realization of the uncertain parameters and a secondlevel optimization problem is integrated into the adaptive robust optimization problem to determine the worst-case realization.

\subsubsection{First-stage constraints}

$$
\begin{gathered}
\sum_{j \in J, m \in M} Y_{i, j, m}=1, \quad \forall i \in I \\
F_{v}\left(Y_{i, j, m}\right)=0, \quad \forall v \in V
\end{gathered}
$$

Binary variable $Y_{i, j, m}$ is introduced to model the selection of technology/process alternatives. $Y_{i, j, m}$ is equal to 1 if technology/process alternative $j$ in section $i$ with 
$m$ parallel operating processes is selected, and 0 otherwise. Constraint (1.21) enforces that one and only one technology/process alternative with a certain number of parallel operating processes is considered in the optimal design. In Eq. (1.22), function $F_{\eta}\left(Y_{i, j, m}\right)$ denotes a set of logic constraints to describe the connections within the network system. By enforcing the logic constraints (1.21) and (1.22), the integrated technology/process alternatives across the network can be selected or rejected simultaneously.

$$
\begin{aligned}
& n u_{m} \cdot C A_{i, j, m} \leq Y_{i, j, m} \cdot u b_{i, j}^{i n}, \quad \forall i \in I, j \in J, m \in M \\
& C A R_{i, j} \leq p r_{i, j} \cdot \sum_{m \in M}\left(n u_{m} \cdot C A_{i, j, m}\right), \quad \forall i \in I, j \in J \\
& n u_{m} \cdot C A_{i, j, m, k}^{\text {product }} \leq Y_{i, j, m} \cdot u b_{i, j}^{\text {product }}, \quad \forall i \in I, j \in J, m \in M, k \in K
\end{aligned}
$$

The upper bound of the capacity of each parallel operating process is given by constraint (1.23), where $n u_{m}$ is the number of parallel operating processes of index $m$ and $u b_{i, j}^{i n}$ is the upper bound of the "in" flow capacity of technology/process alternative $j$ in section $i$. In constraint (1.24), the capacity of each backup process is bounded above by the product of $p r_{i, j}$ and the capacity of all the parallel operating processes for the corresponding technology/process alternative. $p r_{i, j}$ represents the ratio of the allowed capacity of the backup process in technology/process alternative $j$ in section $i$ to that of the corresponding operating process. Constraint (1.25) enforces the upper bound of the normal production level before the 
disruptive events occur. Overall, constraints (1.23)-(1.25) describe the equipment capacities.

$$
\begin{aligned}
& \sum_{n \in N}\left(u_{i, j, n} \cdot W I_{i, j, m, n}\right)=C A_{i, j, m}, \quad \forall i \in I, j \in J, m \in M \\
& \sum_{n \in N} W l_{i, j, m, n}=1, \quad \forall i \in I, j \in J, m \in M \\
& \sum_{n=1}^{|N|-1} E 1_{i, j, m, n}=1, \quad \forall i \in I, j \in J, m \in M \\
& W 1_{i, j, m, 1} \leq E 1_{i, j, m, 1}, \quad \forall i \in I, j \in J, m \in M \\
& W 1_{i, j, m, n} \leq E 1_{i, j, m, n-1}+E 1_{i, j, m, n}, \forall i \in I, j \in J, m \in M, n \in N^{\prime}=\{n: 2 \leq n \leq|N|-1\} \\
& W 1_{i, j, m,|N|} \leq E 1_{i, j, m,|N|-1}, \quad \forall i \in I, j \in J, m \in M \\
& W I_{i, j, m, n} \leq 1, \quad \forall i \in I, j \in J, m \in M, n \in N \\
& \sum_{n \in N}\left(d c p_{i, j, n} \cdot W 1_{i, j, m, n}\right)=C C_{i, j, m}, \quad \forall i \in I, j \in J, m \in M \\
& \sum_{n \in N}\left(u_{i, j, n} \cdot W 2_{i, j, n}\right)=C A R_{i, j}, \quad \forall i \in I, j \in J, \\
& \sum_{n \in N} W 2_{i, j, n}=1, \quad \forall i \in I, j \in J \\
& \sum_{n=1}^{|N|-1} E 2_{i, j, n}=1, \quad \forall i \in I, j \in J \\
& W 2_{i, j, 1} \leq E 2_{i, j, 1}, \quad \forall i \in I, j \in J \\
& W 2_{i, j, n} \leq E 2_{i, j, n-1}+E 2_{i, j, n}, \quad \forall i \in I, j \in J, n \in N^{\prime}=\{n: 2 \leq n \leq|N|-1\} \\
& W 2_{i, j|| N \mid} \leq E 2_{i, j||||-1}, \quad \forall i \in I, j \in J \\
& W 2_{i, j, n} \leq 1, \quad \forall i \in I, j \in J, n \in N
\end{aligned}
$$




$$
\sum_{n \in N}\left(d c p_{i, j, n} \cdot W 2_{i, j, n}\right)=C C R_{i, j}, \quad \forall i \in I, j \in J
$$

In the proposed resilience optimization model, capital costs are evaluated using SOS1 (specially ordered set of type 1) based piecewise linear formulations [268]. Parameters $u_{i, j, n}$ and $d c p_{i, j, n}$ denote the capacity and capital cost of technology/process alternative $j$ in section $i$ for partition points $n$, respectively. By enforcing Eq. (1.28), one and only one SOS1 variable $E 1_{i, j, m, n}$ can be 1 among all the partition points. Based on constraints (1.29)-(1.32), variable $W 1_{i, j, m, n}$ is allowed to vary from 0 to 1 for partition point $n$ and $n+1$, but it must remain 0 for the other partition points. With nonnegative variables $W 1_{i, j, m, n}$ and $W 1_{i, j, m, n+1}$, Eqs. (1.26), (1.27), and (1.33) calculate the capital cost as a linear combination of the capital costs of the two adjacent partition points $n$ and $n+1$. Constraints (1.26)-(1.33) evaluate the capital costs of the operating processes. Similarly, the capital costs of the backup processes are evaluated in a similar way in constraints (1.34)-(1.41).

\subsubsection{Second-stage constraints}

The second-stage constraints describe the recovery model and the mass balance relationships of the operating levels in each time period based on the first-stage decisions and the realization of failed processes after the occurrence of disruptive events.

$$
Z_{i, j, m, t} \leq n u_{m} \cdot Y_{i, j, m}, \quad \forall i \in I, j \in J, m \in M, t \in T
$$




$$
\begin{aligned}
& Z_{i, j, m, 1} \leq n u_{m} \cdot Y_{i, j, m} \cdot\left(1-S I_{i, d}\right), \quad \forall i \in I, j \in J, m \in M, d \in D \\
& Z_{i, j, m, t}=Z_{i, j, m, 1}, \quad \forall i \in I, j \in J, m \in M, t \in T B_{i, j, m} \\
& Z_{i, j, m, t-1} \leq Z_{i, j, m, t}, \quad \forall i \in I, j \in J, m \in M, t \in T A_{i, j, m} \\
& Z_{i, j, m, t} \leq Z_{i, j, m, t^{\prime}}+1, \quad \forall i \in I, j \in J, m \in M,\left(t, t^{\prime}\right) \in\left(T A_{i, j, m}, T L_{i, j, m, t}\right) \\
& Z R_{i, j} \leq \sum_{d \in D}\left(r i_{i, d} \cdot S I_{i, d}\right), \quad \forall i \in I, j \in J
\end{aligned}
$$

$Z_{i, j, m, t}$ denotes the number of available operating processes for technology/process alternative $j$ in section $i$ with $m$ parallel operating processes in time period $t . Z_{i, j, m, t}$ is bounded above by the number of operating processes in each section in constraint (1.42). The number of available operating processes in the first time period $Z_{i, j, m, 1}$ relies on the availability of each process after the occurrence of disruptive events. If the operating processes in section $i$ fail, or $S I_{i, d}$ is equal to one, $Z_{i, j, m, 1}$ should be 0 ; otherwise, $Z_{i, j, m, 1}$ should be bounded above by the number of operating processes in the corresponding section as enforced by constraint (1.43). The time before and after the first operating process is recovered in technology/process alternative $j$ in section $i$ with $m$ parallel processes is $T B_{i, j, m}$ and $T A_{i, j, m}$, respectively. Therefore, the number of available operating processes during $T B_{i, j, m}$ is the same as that in the first time period as described by constraint (1.44). During $T A_{i, j, m}$, the number of available operating processes in time period $t$ is bounded below by the number of available operating processes in time period $t-1$, 
and bounded above by the number of available operating processes in time period $T L_{i, j, m, t}$ plus 1 as described by constraints (1.45) and (1.46), respectively. $Z R_{i, j}$ denotes whether the backup process for technology/process alternative $j$ in section $i$ is available, and $Z R_{i, j}$ is bounded above by the sum of the product of correlation parameter $r i i_{i, d}$ and $S I_{i, d}$ over all disruptive events in constraint (1.47). If the availability of a backup process follows that of the corresponding operating process, the correlation parameter rii,d is equal to 1 ; otherwise, it is equal to 0 . Although $Z_{i, j, m, t}$ and $Z R_{i, j}$ are defined as continuous variables, the optimal solutions of $Z_{i, j, m, t}$ and $Z R_{i, j}$ are integers and the optimal objective function value is not affected by whether $Z_{i, j, m, t}$ and $Z R_{i, j}$ are continuous or integer variables. Constraints (1.42)-(1.47) form the recovery model in the second stage.

$$
\begin{aligned}
& \sum_{k \in K} M_{i, j, k, t}^{i n} \leq \sum_{m \in M}\left(n u_{m} \cdot C A_{i, j, m}\right), \quad \forall i \in I, j \in J, t \in T \\
& \sum_{k \in K} M_{i, j, k, t}^{i n} \leq \sum_{m \in M}\left(Z_{i, j, m, t} \cdot C A_{i, j, m}\right)+Z R_{i, j} \cdot C A R_{i, j}, \quad \forall i \in I, j \in J, t \in T \\
& \sum_{k \in K} M_{i, j, k, t}^{\text {product }} \leq \sum_{m \in M, k \in K}\left(n u_{m} \cdot C A_{i, j, m, k}^{\text {product }}\right), \quad \forall i \in I, j \in J, t \in T
\end{aligned}
$$




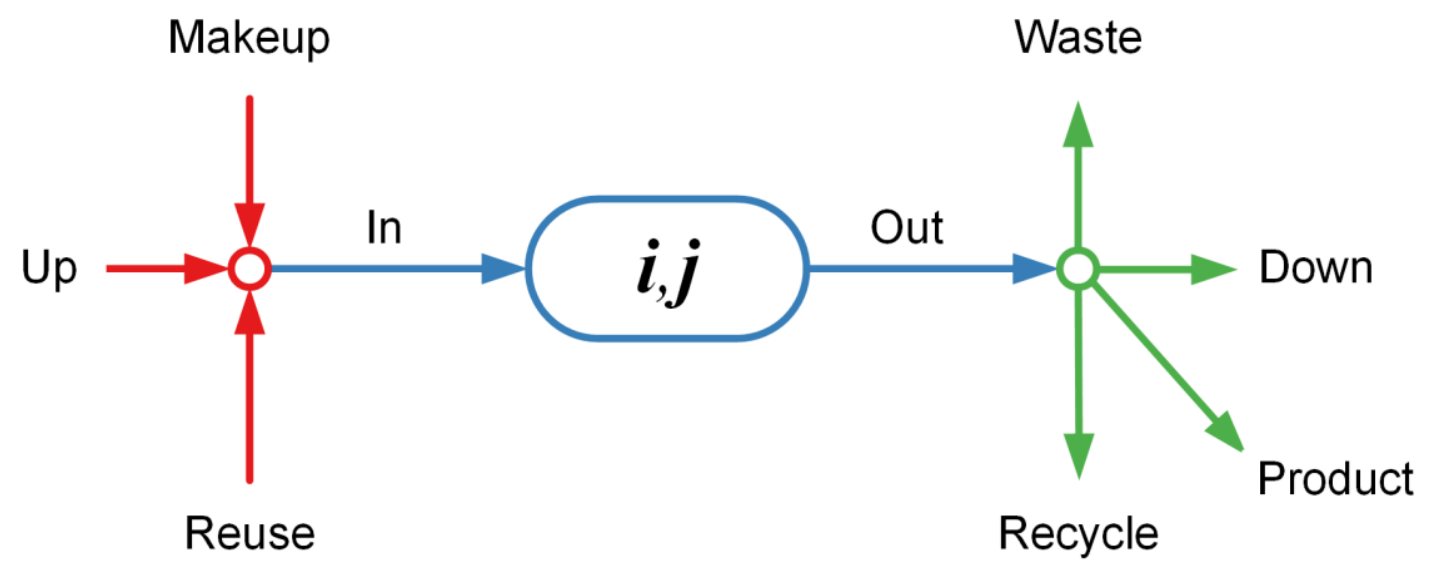

Figure 61. General mass balance model for technology/process alternative $j$ in section $i$.

Following a general mass balance model in Figure 61, nine types of flows ("up", “makeup", "reuse", "in”, “out”, “down”, "recycle", "product”, and “waste” flows) are introduced for each technology/process alternative [21]. In constraint (1.48), the operating levels of the "in" flows are bounded above by the capacity of all the operating processes for technology/process alternative $j$ in section $i$. Additionally, it is bounded above by the sum of the capacities of the available operating processes and backup process as enforced by constraint (1.49). It is noted that variables $C A_{i, j, m}$ and $C A R_{i, j}$ are first-stage decision variables and they are known parameters in the third-level optimization problem. In constraint (1.50), the operating levels of the "product" flows are bounded above by the total production level without disruptive events for technology/process alternative $j$ in section $i$.

$$
M_{t}^{\text {feed }} \leq u b^{\text {feed }}, \quad \forall t \in T
$$




$$
\begin{aligned}
& \sum_{j \in J} M_{1, j, k, t}^{u p}=w f_{k}^{\text {feed }} \cdot M_{t}^{\text {feed }}, \quad \forall k \in K, t \in T \\
& \sum_{j \in J} M_{i, j, k, t}^{u p}=\sum_{j \in J} M_{i-1, j, k, t}^{\text {down }}, \quad \forall i \geq 2, k \in K, t \in T \\
& \sum_{j \in J} M_{i, j, k, t}^{\text {reuse }}=\sum_{i^{\prime} \in R E_{i}, j \in J} M_{i^{\prime}, j, k, t}^{\text {recycle }}, \quad \forall i \in I, k \in K, t \in T \\
& M_{i, j, k, t}^{u p}+M_{i, j, k, t}^{\text {reuse }}+M_{i, j, k, t}^{\text {makeup }}=\sum_{k^{\prime} \in K}\left[p_{i, j, k, k^{\prime}}^{\text {makeup }} \cdot\left(M_{i, j, k^{\prime}, t}^{\text {up }}+M_{i, j, k^{\prime}, t}^{\text {reuse }}\right)\right], \quad \forall i, j, k, t \\
& M_{i, j, k, t}^{\text {in }}=M_{i, j, k, t}^{u p}+M_{i, j, k, t}^{\text {reuse }}+M_{i, j, k, t}^{\text {makeup }}, \quad \forall i \in I, j \in J, k \in K, t \in T
\end{aligned}
$$

The feedstock flow rate $M_{t}^{\text {feed }}$ is bounded above by $u b^{\text {feed }}$. The "up" flows in the first section of the network are from the feedstock of the entire network. In contrast, the "up" flows in the remaining sections are from the "down" flows in the previous section. The "reuse" flows gather relevant "recycle" flows from other sections of the system. The "makeup" flows account for the consumption of other materials in addition to the feedstock of the network system. $p_{i, j, k, k^{\prime}}^{\text {makeup }}$ denotes the ratio of the flow rate of species $k$ to the flow rate of species $k$ ' in the "in" flow of technology/process alternative $j$ in section $i$. The "up", "reuse" and "makeup" flows in each section form the corresponding "in" flow. Constraints (1.51)-(1.56) describe the inlet converging step of the general mass balance model.

$$
M_{i, j, k, t}^{i n}+\sum_{k^{\prime} \in K}\left(s c_{i, j, k, k^{\prime}} \cdot M_{i, j, k^{\prime}, t}^{i n}\right)=M_{i, j, k, t}^{\text {out }}, \quad \forall i \in I, j \in J, k \in K, t \in T
$$


The conversion step of the general mass balance model is enforced by constraint (1.57), where $s c_{i, j, k, k^{\prime}}$ denotes the stoichiometric coefficient of species $k$ based on species $k^{\prime}$ in technology/process alternative $j$ in section $i$.

$$
\begin{aligned}
& M_{i, j, k, t}^{\text {out }}=M_{i, j, k, t}^{\text {down }}+M_{i, j, k, t}^{\text {recyle }}+M_{i, j, k, t}^{\text {product }}+M_{i, j, k, t}^{\text {waste }}, \quad \forall i \in I, j \in J, k \in K, t \in T \\
& M_{i, j, k, t}^{\text {down }}=s f_{i, j, k}^{\text {down }} \cdot M_{i, j, k, t}^{\text {out }}, \quad \forall i \in I, j \in J, k \in K, t \in T \\
& M_{i, j, k, t}^{\text {recyle }}=s f_{i, j, k}^{\text {recycle }} \cdot M_{i, j, k, t}^{\text {out }}, \quad \forall i \in I, j \in J, k \in K, t \in T \\
& M_{i, j, k, t}^{\text {product }}=s f_{i, j, k}^{\text {product }} \cdot M_{i, j, k, t}^{\text {out }}, \quad \forall i \in I, j \in J, k \in K, t \in T \\
& \sum_{i \in I, j \in J} M_{i, j, k, t_{n}}^{\text {product }} \geq p_{k}^{\text {demand }}, \quad \forall k \in K
\end{aligned}
$$

In constraint (1.58), the "out" flow of each technology/process alternative $j$ in section $i$ is separated into a "down" flow, a "recycle" flow, a "product" flow, and a "waste" flow. In constraints (1.59)-(1.61), sf down,$s f_{i, j, k}^{\text {recyle }}$ and $s f_{i, j, k}^{\text {product }}$ are split fractions of the "down", "product" and the "waste" flow, respectively. Constraint (1.62) requires that the operating level of each product at the end of the time horizon is no less than the demand of that product. Overall, constraints (1.58)(1.61) describe the outlet separation step of the general mass balance model.

The proposed multiobjective two-stage ARMIFP model has a three-level structure. Integer variables are introduced in the first-level problem to select the technology/process alternatives and facilitate the process designs, as well as in the second-level problem to determine the worst-case realization of failed processes 
after the occurrence of disruptive events. The other decision variables, such as equipment capacities, capital costs, and operating levels in each time period, are continuous variables. Additionally, the only nonlinear term in the optimization model is the fractional objective function for the proposed quantitative measure of resilience. Therefore, the resulting problem is a multiobjective multilevel nonconvex mixed-integer nonlinear programming (MINLP) problem.

\subsubsection{Tailored solution algorithm}

Since the proposed multiobjective two-stage ARMIFP model has a multilevel structure, it cannot be handled directly by any off-the-shelf optimization solvers. Additionally, due to the combinatorial nature and nonconvexity stemming from the mixed-integer terms and the fractional objective function, the optimization problem is challenging to solve. To tackle the computational challenge, we employ a tailored optimization algorithm to efficiently solve this ARMIFP problem [267]. Specifically, the optimization algorithm integrates two state-of-the-art algorithms, including the inexact parametric algorithm for handling the fractional objective function and the column-and-constraint generation algorithm for solving an auxiliary two-stage adaptive robust mixed-integer linear programming (MILP) problems [202, 228]. 
ARMIFP:

$$
\begin{aligned}
& \max _{X l \in C l} \min _{S I \in U} \max _{X 2 \in O} \frac{f n(X 2)}{f d(X 1)} \\
& \min _{X l \in C l} f_{C}(X 1)
\end{aligned}
$$

Conventional design and operations problem: $\min _{X l \in C l} f_{c}(X 1)$

Auxiliary problem:

$$
P(r)=\max _{X I \in C l}-r \cdot f d(X 1)+\min _{S I \in U} \max _{X 2 \in O} f n(X 2)
$$

Reformulated master problem: $\quad \max _{(X I, X 2, \eta) \in C M}-r \cdot f d(X 1)+\eta$

\section{Reformulated subproblem: $\min _{(S I, P) \in C S} S O$}

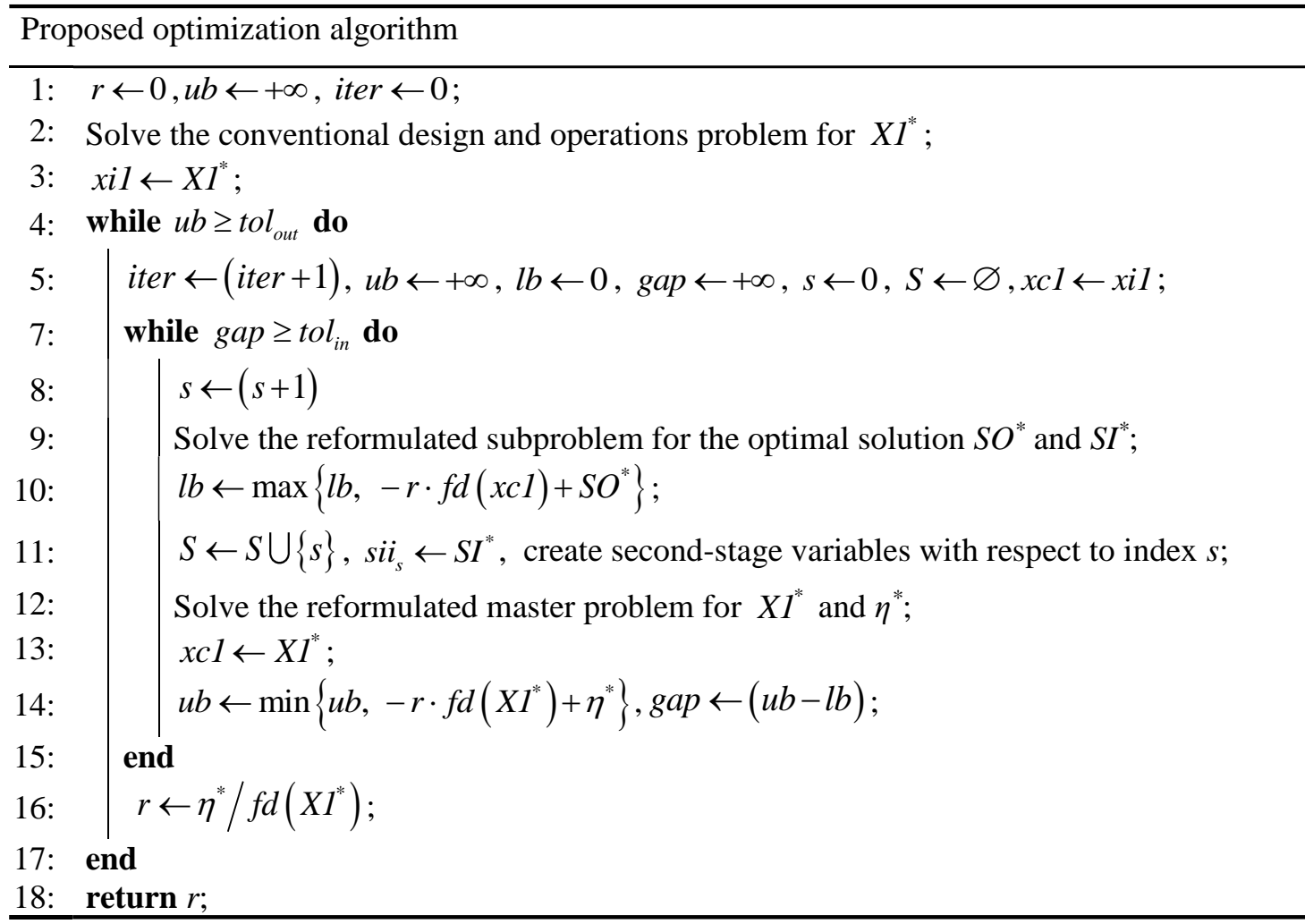

Figure 62. The pseudo code of the proposed optimization method that integrates the inexact parametric algorithm and the column-and-constraint generation algorithm. 
For illustration purposes, the compact forms of involved models are introduced. $X 1$ and $X 2$ denote the first-stage and second-stage variables, respectively. $f_{c}(X 1)$, $f d(X 1)$, and $f n(X 2)$ denote the economic objective function, the denominator of the resilience measure, and the numerator of the resilience measure, respectively. $\eta$ represents the second-stage objective function in the reformulated master problem, and $S O$ represents the objective function of the subproblem. $C 1, U, O, C M$, and $C S$ denote the feasible regions of the first-level optimization problem, second-level optimization problem, third-level optimization problem, reformulated master problem, and reformulated subproblem, respectively. The detailed model formulations of the conventional design and operations problem, the auxiliary parametric problem, the master problem, and the subproblem are given in Appendices A, B, C, and D, respectively.

The proposed optimization algorithm employs the inexact parametric algorithm in the outer loop to tackle the computational challenge stemming from the fractional objective function. Instead of solving the original optimization problem with the fractional objective function directly, we introduce an auxiliary parameter $r$ and an auxiliary parametric problem $P(r)$. Following the $\varepsilon$-constraint method [199], the economic objective function in the original optimization problem is converted to Constraint (1.B2) (details are given in Appendix B). The optimal solution of the 
orginal optimization problem is identical to the optimal solution of the auxiliary parametric problem with the parameter $r^{*}$ such that $P\left(r^{*}\right)=0$ [202]. In each iteration of the inexact parametric algorithm, we need to solve a two-stage adaptive robust MILP problem $P(r)$, which cannot be tackled directly by any off-the-shelf optimization solvers. In the inner loop of the proposed optimization algorithm, a master problem and a subproblem of $P(r)$ are developed and solved iteratively following the column-and-constraint generation algorithm [228]. This solution algorithm is guaranteed to converge within finite iterations [267]. A conventional design and operations problem that minimizes the total capital cost without disruptive events is solved after the initialization step, and the optimal first-stage solutions are used by the first subproblem in each inner loop. The pseudo code of the optimization algorithm is shown above, and the flowchart of the algorithm is presented later. The iteration counters in the outer loop and the inner loop are denoted as iter and $s$, respectively. The optimality tolerance in the outer loop and the inner loop are denoted as tolout and tolin, respectively. $S O^{*}$ and $M O^{*}$ represent the optimal objective function values of the subproblem and the master problem, respectively, while $u b$ and $l b$ represent the upper bound and the lower bound in the inner loop, respectively. gap denotes the optimality gap in the inner loop. It is noted that $u b=+\infty$ is presented in the initialization step of the pseudo code but not 
in the flowchart because the loop condition of the pseudo code comes ahead of solving any optimization problems.

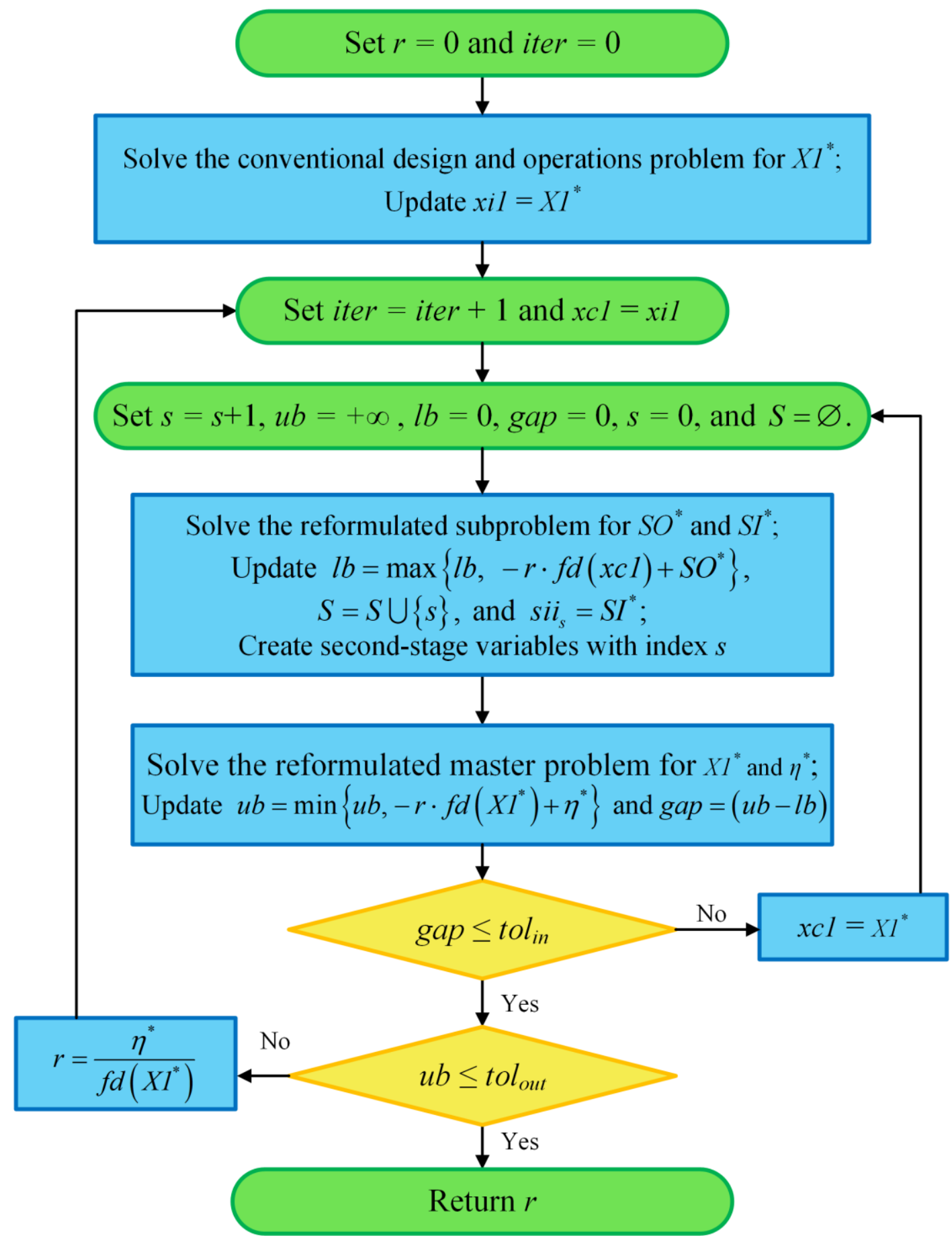


Figure 63. Flowchart of the proposed optimization algorithm.

\subsection{Applications of the general framework for resilience optimization}

\subsubsection{Application 1: Chemical process network design and planning}

The application of the proposed resilience optimization framework is first illustrated thorough the resilient design and planning of a chemical process network with ten chemicals and six processes as shown in Figure 64 [200]. The feedstock materials include acetylene, propylene, benzene, and nitric acid; the products include acetaldehyde, acrylonitrile, isopropanol, phenol, acetone, and cumene. The correlation parameter ri,d is equal to 1 for all processes and disruptive events. The tolerance level $\varepsilon_{d}$ is equal to $5 \%$ for all disruptive events. We consider 4 case studies of this application. Case studies 1-3 involve only one disruptive event, and case study 4 involves two disruptive events. The numbers of failed processes in case studies $1-3$ are 2,4 , and 6 , respectively. In case study 4 , the numbers of failed processes of the first and second disruptive events are 3 and 2, respectively. The data on recovery time are provided in Appendix E.

All computational experiments are performed on a DELL OPTIPLEX 7040 desktop with Intel(R) Core(TM) i7-6700 CPU @ 3.40GHz and 32 GB RAM. The solution procedure is coded in GAMS 24.8.5 [203], with CPLEX 12.7 used as the MILP 
solver. The relative optimality tolerances for the inexact parametric algorithm and the column-and-constraint generation algorithm are $10^{-6}$.

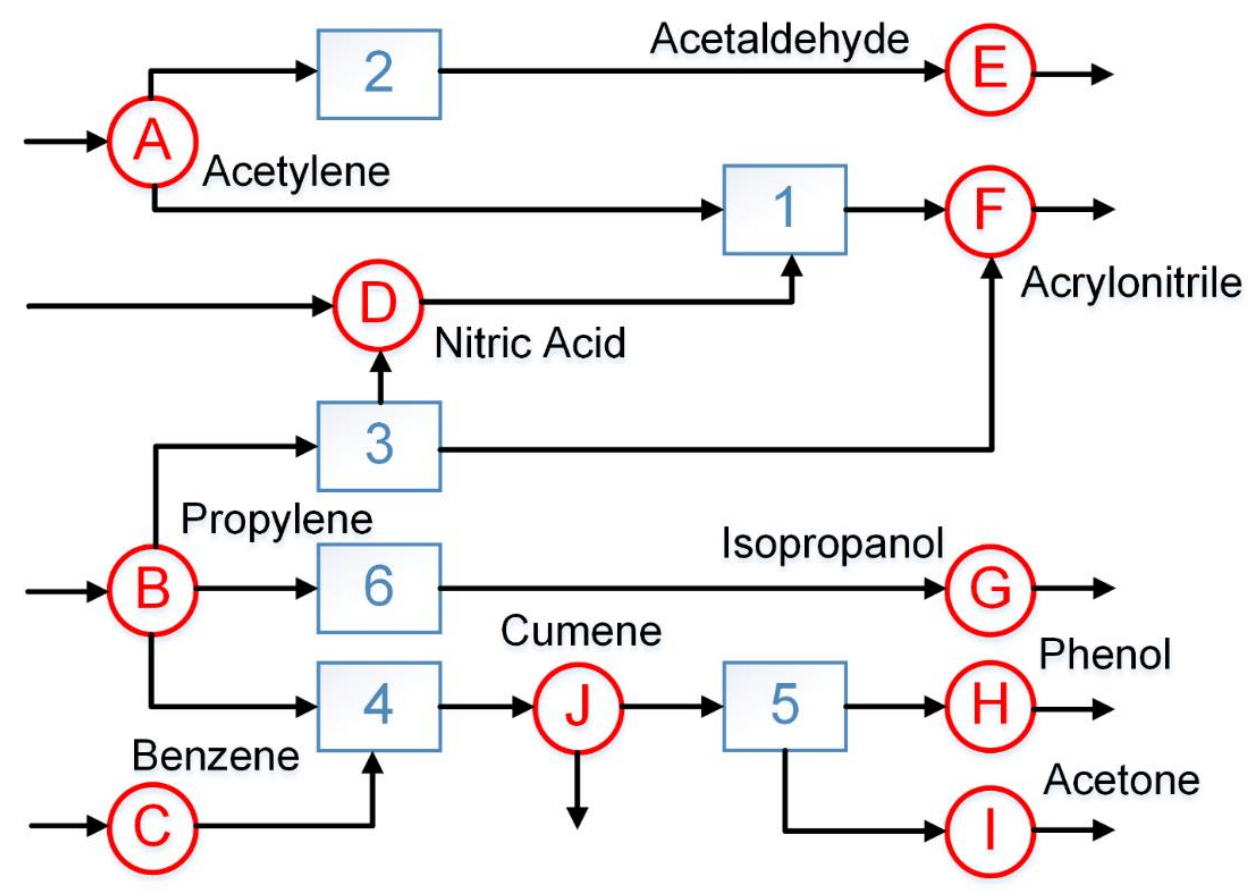

Figure 64. Chemical process network in the first application.

Table 12. Computational times for the instances of the optimal solutions A, B, C, and D.

\begin{tabular}{cccc}
\hline Instances & $\begin{array}{c}\text { Number of outer } \\
\text { iterations }\end{array}$ & $\begin{array}{c}\text { Computational } \\
\text { times for solving } \\
\text { master problems } \\
\text { (CPUs) }\end{array}$ & $\begin{array}{c}\text { Computational } \\
\text { times for solving } \\
\text { subproblems } \\
\text { (CPUs) }\end{array}$ \\
\hline A & 2 & 31 & 1 \\
B & 2 & 13 & 1 \\
C & 2 & 27 & 1 \\
D & 2 & 79 & 2 \\
\hline
\end{tabular}

In this application, the subproblem of all case studies and instances consist of 6 integer variables, 1,766 continuous variables, and 1,690 constraints. Depending on 
the number of inner iterations, the size of the master problem can increase from 132 integer variables, 3,547 continuous variables, and 3,243 constraints, to 732 integer variables, 12,182 continuous variables, and 14,468 constraints. As shown in Table 12, four instances in the first application can be solved in less than $2 \mathrm{~min}$. The four instances are all based on case study 1 . The computational times for solving the master problems are significantly longer than the computational times for solving the corresponding subproblems. The short overall computational times indicate that the proposed optimization algorithm is very efficient for the proposed bi-criterion two-stage ARMIFP problems. 


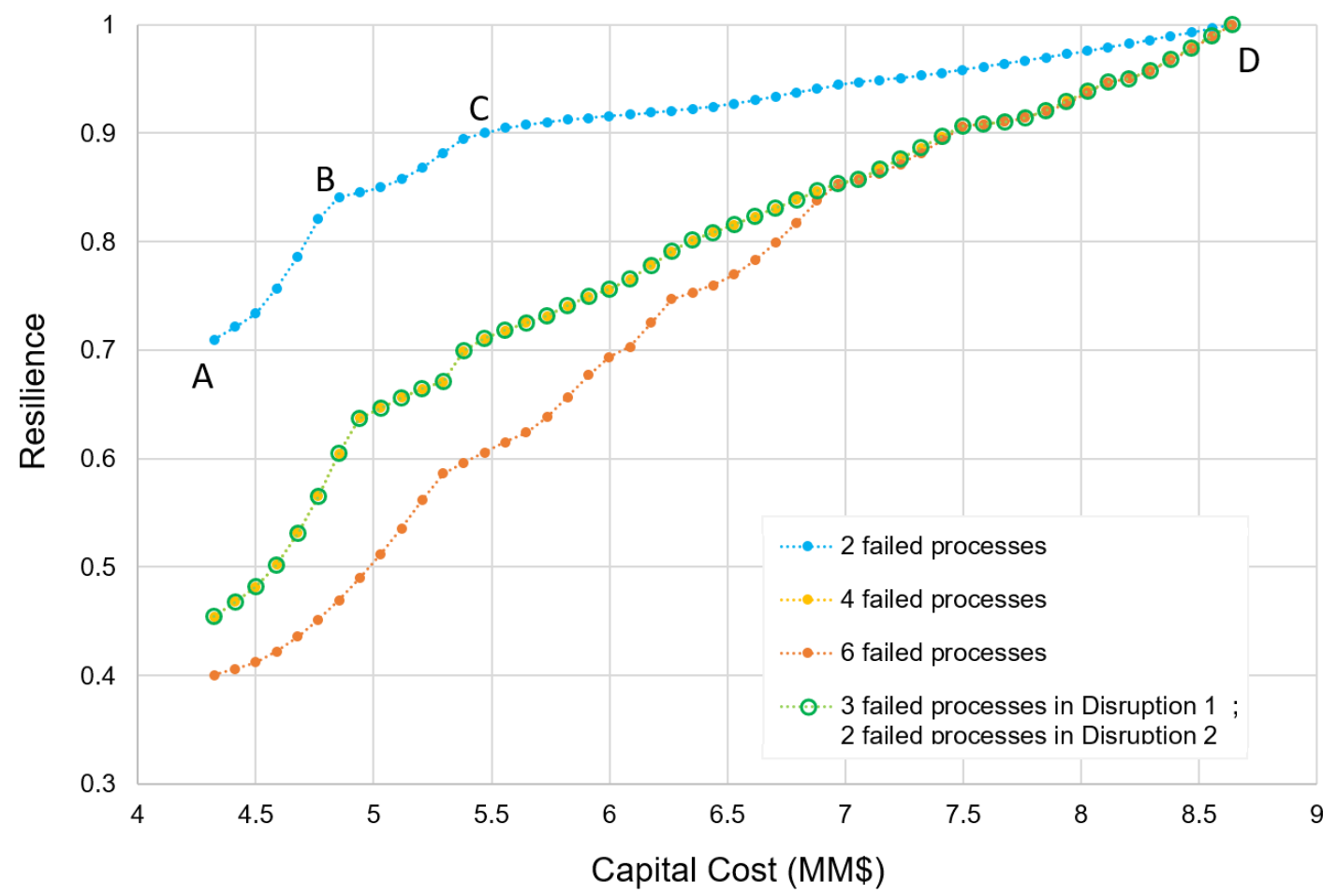

Figure 65. Pareto-optimal curves of the application for chemical process network design and planning.

The optimal solutions of the multiobjective two-stage ARMIFP problems can be plotted as Pareto-optimal curves in Figure 65. Each point on the Pareto-optimal curves corresponds to an optimal solution of the optimization problems. On the Pareto-optimal curves, an increase in resilience corresponds to an increase in the total capital cost. For case study 1 , the most resilient solution demonstrates a resilience of 1 and a capital cost of $\$ 8.64 \mathrm{MM}$, while the most cost-effective solution demonstrates a resilience of 0.71 and a capital cost of $\$ 4.32 \mathrm{MM}$. Both optimal solutions employ the same operating processes, but the minimum capital 
cost of the most resilient solution is twice of that of the most cost-effective solution. It is noted that the most resilient solution includes a set of backup processes with the same capacities of the corresponding operating processes, while no backup process is built for the most cost-effective solution. The optimal capital cost ranges from $\$ 4.32 \mathrm{MM}$ to $\$ 8.64 \mathrm{MM}$ across all the case studies. If the optimal capital cost is fixed, the optimal resilience decreases as more failed processes are considered in the optimization problem. The Pareto-optimal curve in case study 2 overlaps with that in case study 4 , because the total numbers of failed processes adjusted by the tolerance level are the same in these two case studies. Similar to budge of uncertainty, the total number of failed processes and the tolerance level are adjustable parameters to control the level of conservatism of the robust optimal solution. In Figure 66, when the capital cost is fixed at $\$ 6 \mathrm{MM}$, a larger number of failed processes and a smaller tolerance level correspond to a smaller and more conservative resilience result. 


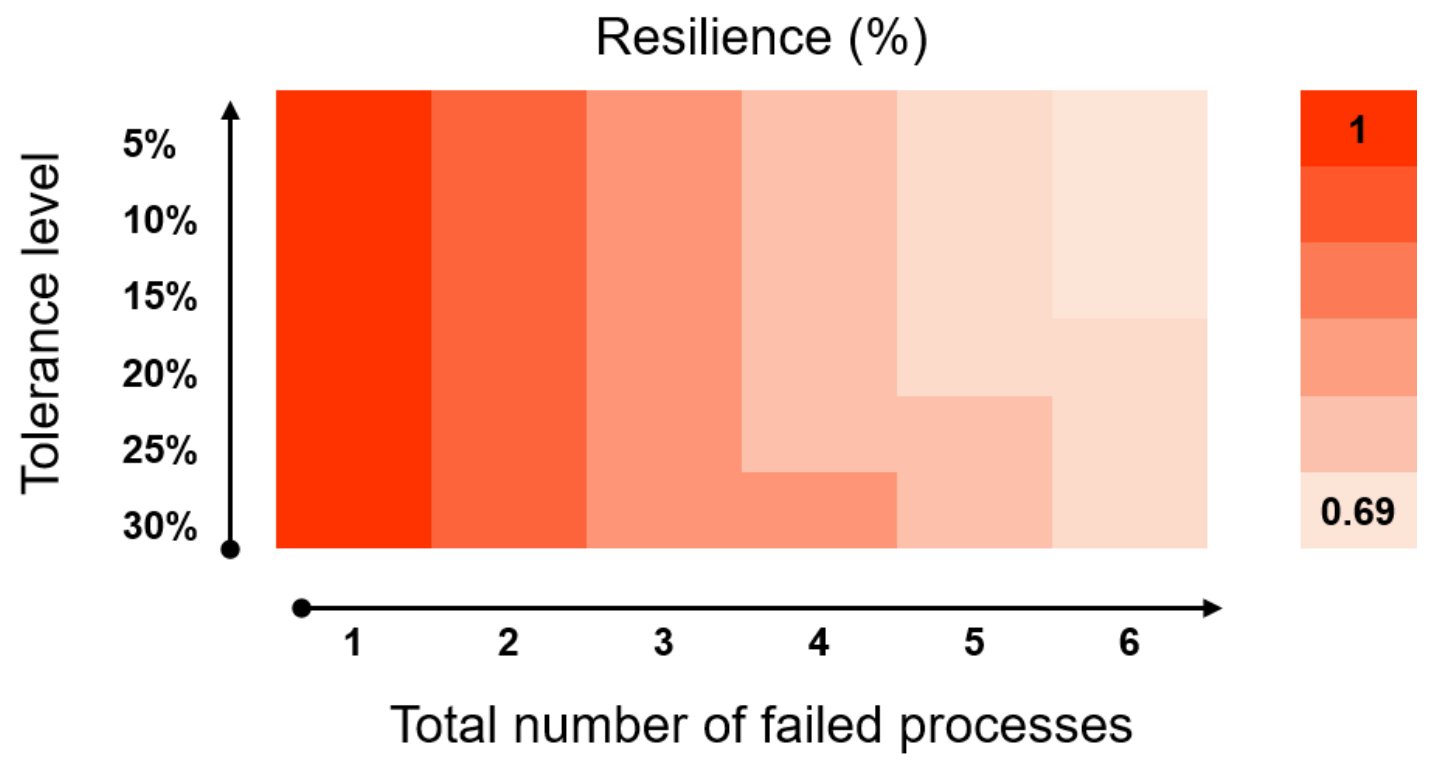

Figure 66. Heatmap of the optimal resilience results with a capital cost of $\$ 6 \mathrm{MM}$, various numbers of failed processes, and various tolerance levels.

Each point on a Pareto-optimal curve corresponds to an optimal process design.

Figure 67 presents the optimal capital costs as well as the worst-case realization of the optimal solutions A, B, C, and D in Figure 65. To satisfy the product demands, the optimal solutions $\mathrm{A}, \mathrm{B}$, and $\mathrm{D}$ select the same processes with the same equipment capacities of the operating processes. The difference lies in the capacities of the backup processes. There is no backup process in the optimal solution A in order to minimize the total capital cost. With a higher capital investment, a backup process is built for process 4 in the optimal solution $B$. Although the capacity of this backup process is smaller than that of the corresponding operating process, it effectively increases the worst-case operating 
levels from 230 ton/day to 330 ton/day in time periods $1-3$. As an extreme case of the optimal solution $\mathrm{B}$, the optimal solution D establishes backup processes for all the operating processes and the capacities of the backup processes are the same as the corresponding operating processes. In the optimal network designs of solutions A, B, and D, only process 1 is employed to produce acrylonitrile. A different process design is selected for the optimal solution $\mathrm{C}$ and both process 1 and process 3 are employed to produce acrylonitrile. Accordingly, the capacity of process 1 in the optimal solution C is lower than those in the optimal solutions A, B, and D. 


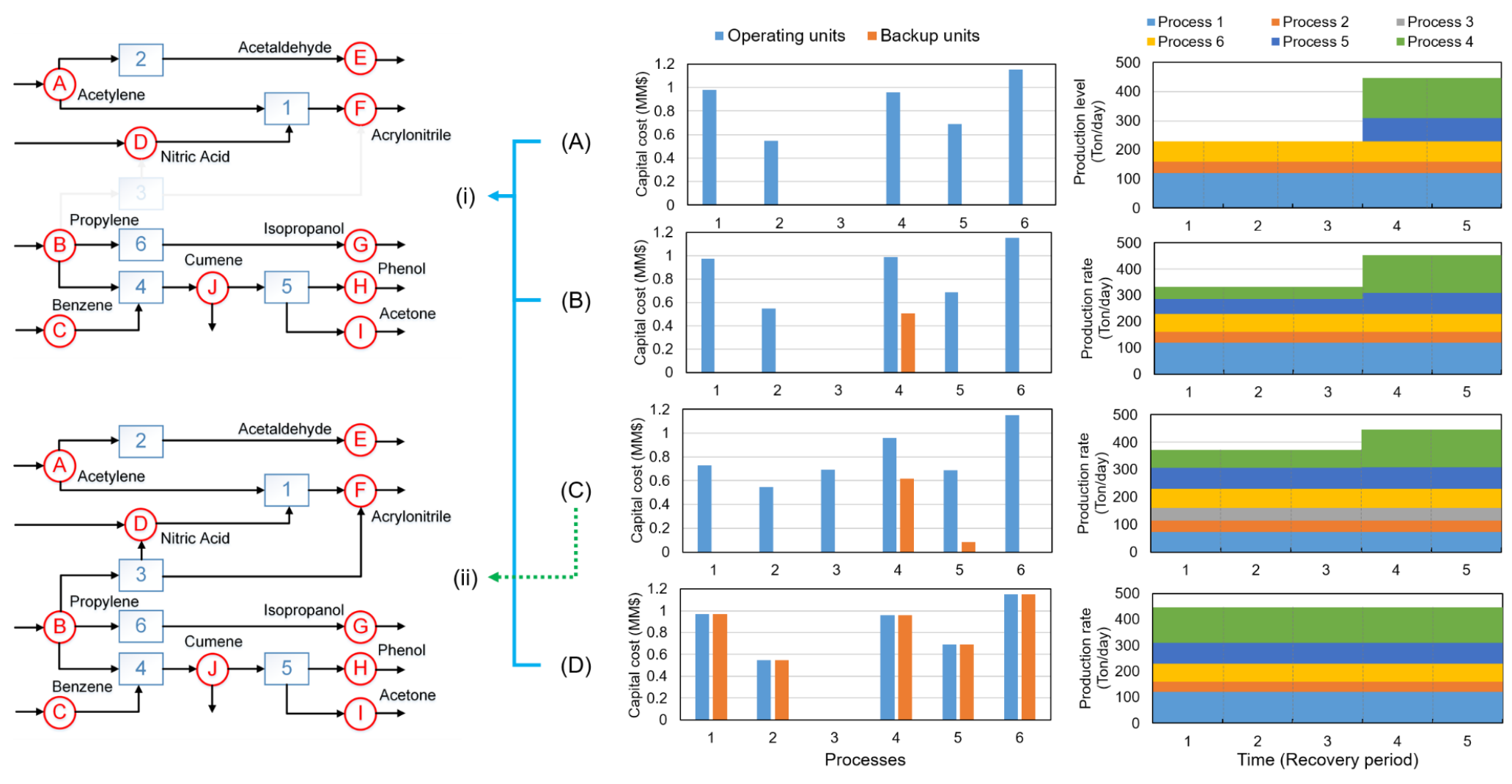

Figure 67. Capital cost breakdowns and worst-case realizations of four optimal solutions. Network design (i) corresponds to the optimal solutions A, B, and D in Figure 65. Network design (ii) corresponds to the optimal solutions C in Figure 65. 
Figure 67 also displays the worst-case realizations for the corresponding optimal solutions. Process 4 is identified as the failed process in the optimal solutions A, B, and C. Because the product of process 4 is the feed of process 5 and no backup process is built in the optimal solution A, both processes produce nothing in time periods 1-3. As a backup process is established for process 4 in the optimal solution $\mathrm{B}$, it instantly recovers a portion of the lost capacities. Cumene from process 4 is then converted to phenol and acetone in process 5 . Therefore, the gap between the production rate and the capacity in the optimal solution B is smaller than that in the optimal solution A. As the capacity of the backup process in the optimal solution $\mathrm{C}$ is higher than that in the optimal solution $\mathrm{B}$, the production rate in process 5 becomes higher and the resilience is also higher. The worst-case realization of the optimal solution $\mathrm{D}$ shows that no matter what process is affected by the disruptive event, the demand can be always satisfied.

\subsubsection{Application 2: Superstructure optimization of shale gas processing and NGLs recovery processes}

The application of the proposed resilience optimization framework is then illustrated through the superstructure optimization of shale gas processing and NGLs recovery processes $[26,110,111,116]$. We develop a superstructure that integrates 108 possible process designs for shale gas processing and natural gas liquids recovery processes. The superstructure consists of four sections, namely 
acid gas removal (green), dehydration (yellow), NGLs recovery (blue), and sulfur recovery (purple). The considered technology and process alternatives in each section are presented in Figure 68. The process flowsheet of the superstructure is shown in Figure 69.

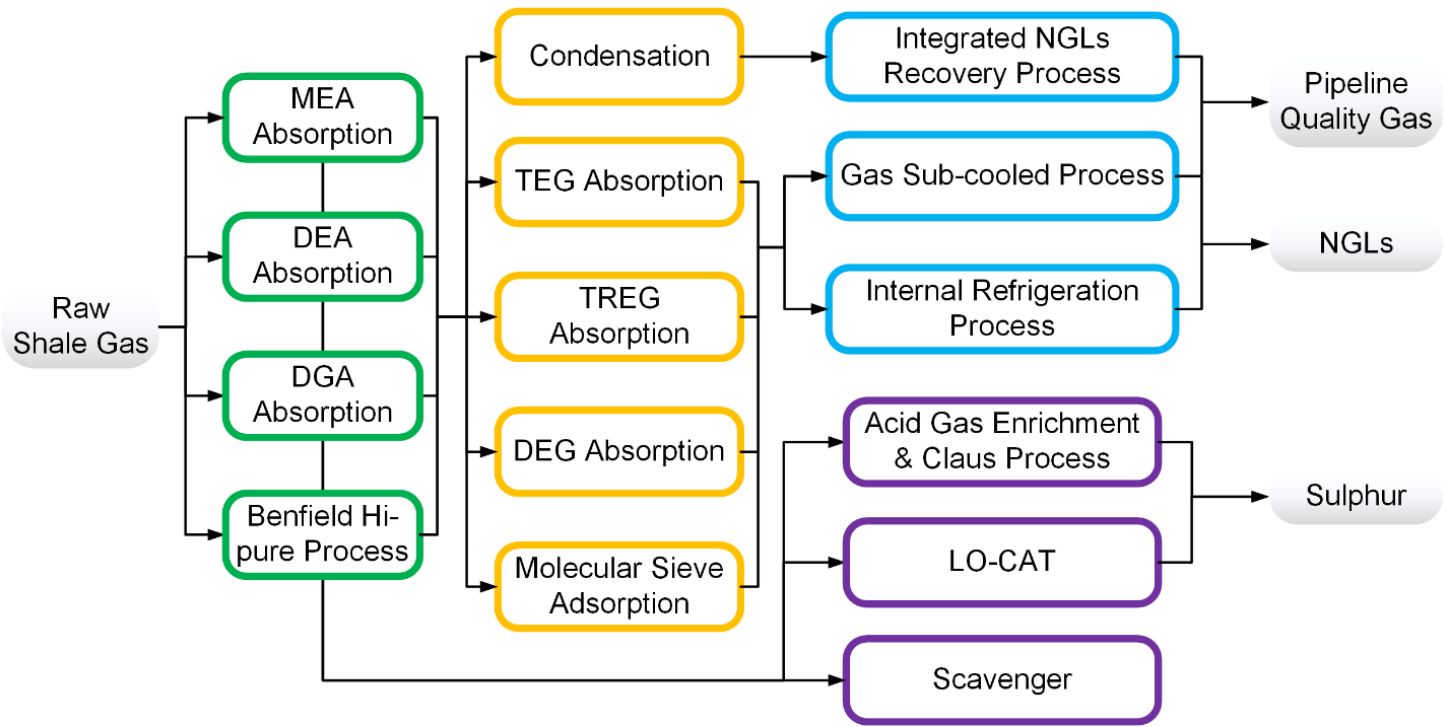

Figure 68. Technology and process alternatives in the superstructure of shale gas processing and NGLs recovery processes.

Similar to the settings in the first application, the correlation parameter $r i, d$ is equal to 1 and the tolerance level $\varepsilon_{d}$ is equal to $5 \%$ for all disruptive events. A total of 4 case studies are considered: Case studies 1-3 involve only one disruptive event with 2, 4, and 6 failed processes, respectively, while Case study 4 involves two disruptive events with 3 and 2 failed processes in the first and second disruptive events, respectively. The resulting subproblem consists of 4 integer variables, 8,581 continuous variables, and 9,232 constraints. The size of the master problem can increase from 330 integer variables, 17,523 continuous variables, and 15,889 
constraints, to 1,530 integer variables, 55,163 continuous variables, and 55,013 constraints. In all the instances, it takes at most 10 inner iterations and 5 outer iterations for the proposed optimization algorithm to converge to the global optimal solution. The upper bound and the lower bound of each iteration for the instance of the optimal solution $G$ is presented in Figure 70. It takes 2 outer iterations for the inexact parametric algorithm to find the root of $P(r)=0$, and in each inner loop, the gap between the upper and lower bounds closes in 5 iterations. Figure 70 illustrates the algorithm features which integrate the parametric algorithm in the outer loop and the column-and-constraint generation algorithm in the inner loop. 


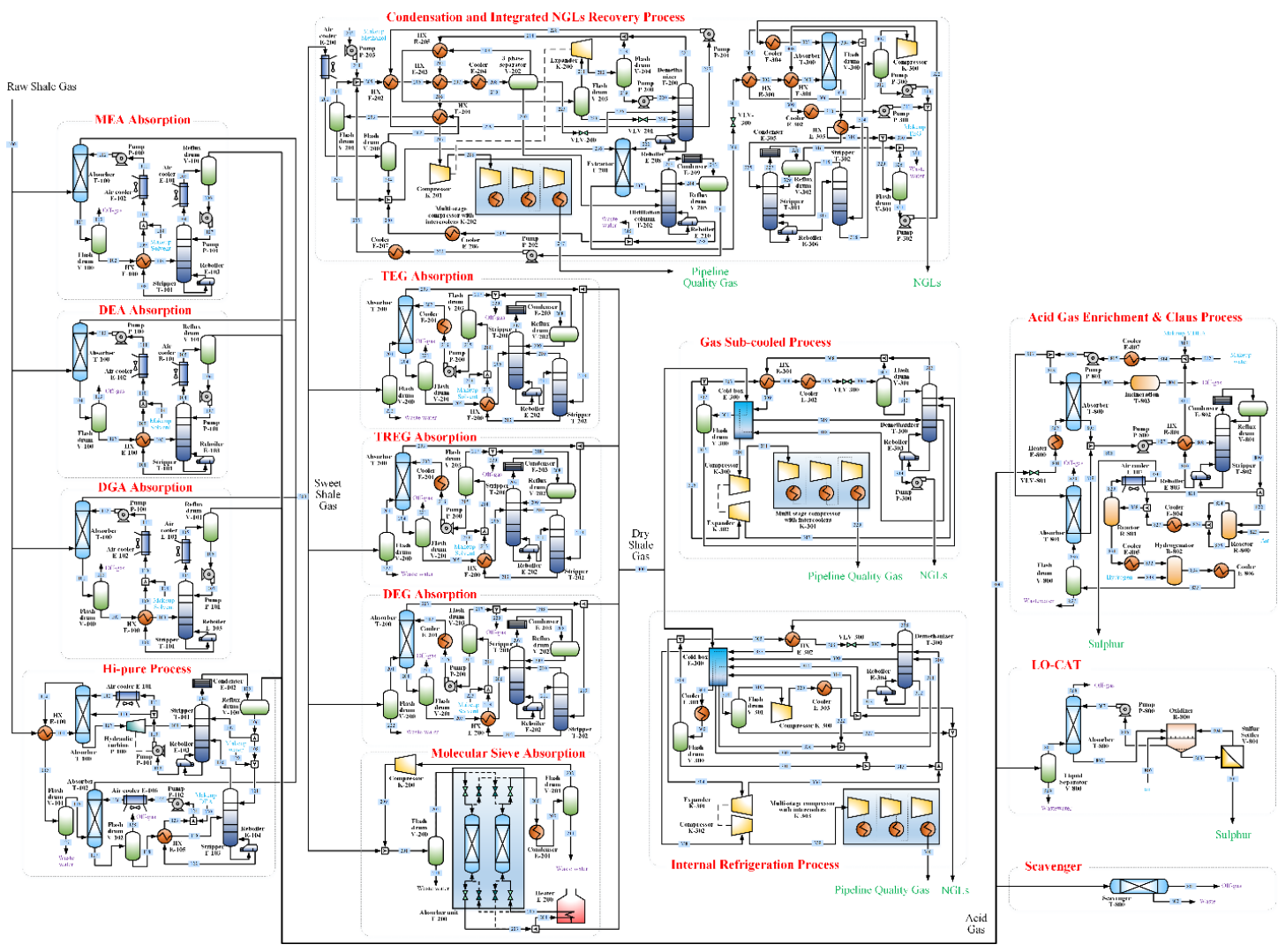

Figure 69. Process flowsheet of the superstructure for shale gas processing and NGLs recovery processes. 


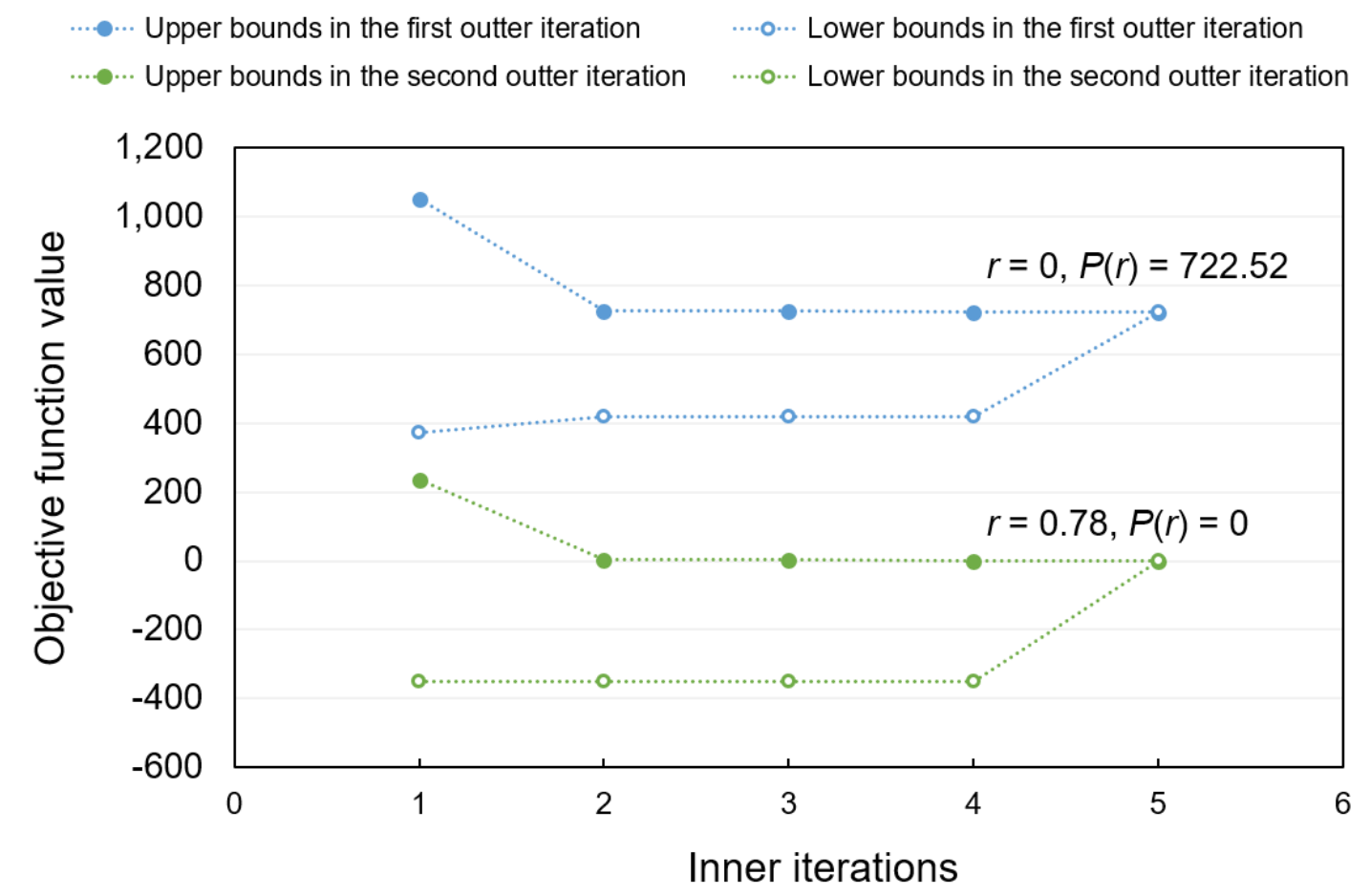

Figure 70. Upper and lower bounds of each iteration for the instance of the optimal solution G.

Table 13. Computational times for the instances of the optimal solutions E, F, G, and $\mathrm{H}$.

\begin{tabular}{ccc}
\hline Instances & $\begin{array}{c}\text { Computational times for solving } \\
\text { master problems (CPUs) }\end{array}$ & $\begin{array}{c}\text { Computational times for solving } \\
\text { subproblems (CPUs) }\end{array}$ \\
\hline E & 238 & 2 \\
F & 716 & 2 \\
G & 571 & 1 \\
H & 494 & 1 \\
\hline
\end{tabular}

The computational times for the instances of the optimal solutions E, F, G, and H are presented in Table 13. The optimization problem in the second application can be solved in less than $12 \mathrm{~min}$. Given that the proposed problems cannot be handled directly by any off-the-shelf optimization solvers, the reasonable computational 
times and the small number of iterations illustrate the computational efficiency of the proposed optimization algorithm.

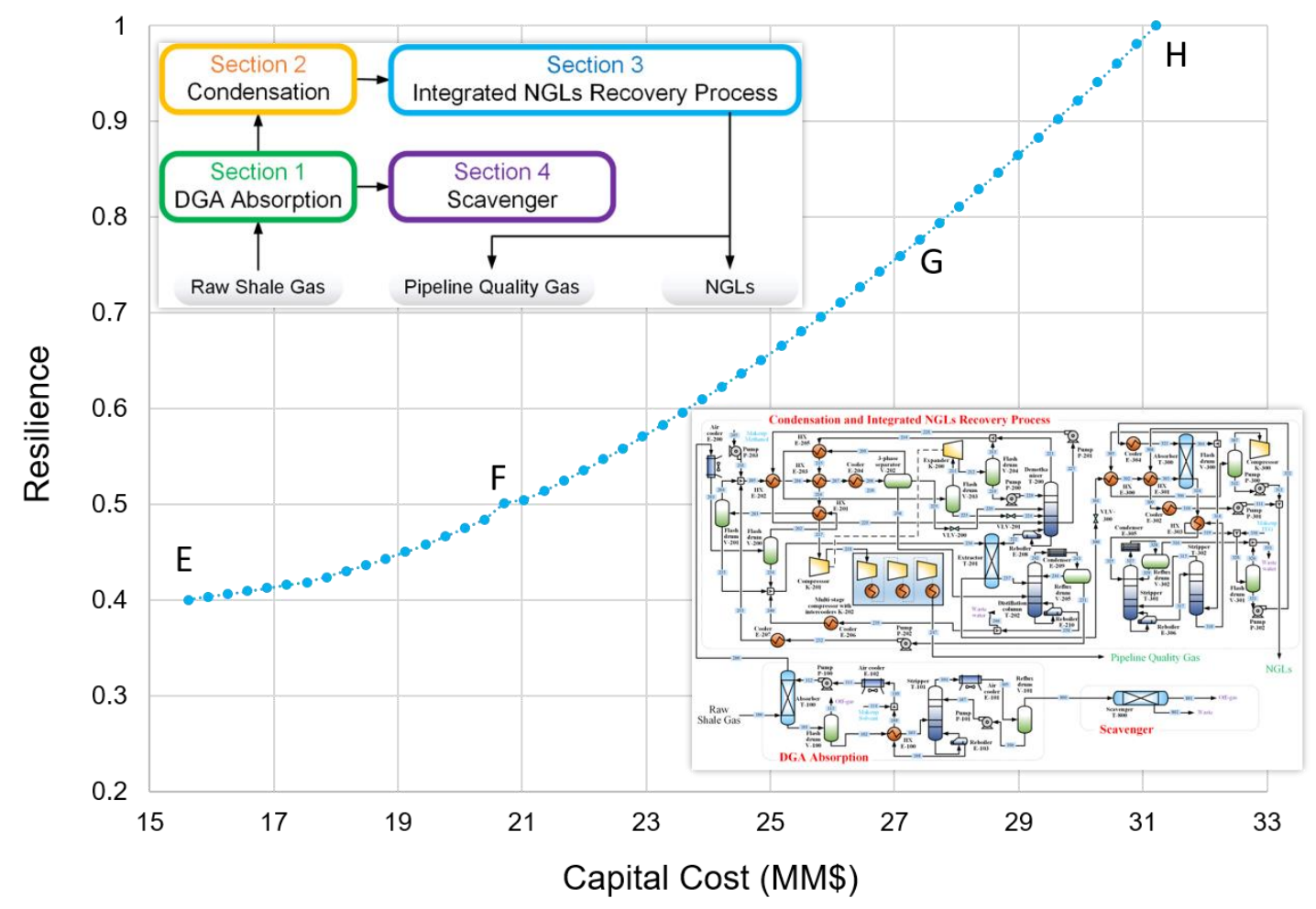

Figure 71. Pareto-optimal curve and optimal process design of the superstructure optimization application.

The optimal solutions are plotted in the Pareto-optimal curve in Figure 71. Since the shale gas processing and NGLs recovery processes form a tandem system, the entire system will be disrupted even if only one process in the system fails. In order to increase the resilience, backup processes must be built for all the operating processes simultaneously regardless of the number of failed processes. As a result, the optimal solutions and optimal objective function values are the same across all the cases studies, and only one Pareto-optimal curve is exhibited in Figure 71. The 
most resilient solution demonstrates a resilience of 1 and a capital cost of $\$ 31.22$ MM, while the most cost-effective solution demonstrates a resilience of 0.4 and a capital cost of $\$ 15.61 \mathrm{MM}$. A tradeoff can be seen between the two competing objective functions. A resilience of 1 for the most resilient solution means that the production rate is not affected by the disruptive events, but such an ideal performance corresponds to the highest capital cost, which is twice the cost of the most cost-effective solution.

All the optimal solutions on the Pareto-optimal curve select the same technologies/processes, namely diglycolamine (DGA) adsorption in the acid gas removal section, condensation in the dehydration section, integrated NGLs recovery process in the NGLs recovery section, and scavenger in the sulfur recovery section. The selected technologies and the corresponding flowsheet are also presented in Figure 71. This result provides a good example of selecting the most suitable technology to maximize resilience. On the same basis, the resilience would be higher if additional products can be generated in the same process system. However, the technology alternatives that generate additional sulfur in the sulfur recovery section are not selected by the optimal process design, because of the relatively high capital costs of these technology alternatives. Although the selected scavenger process does not generate a sulfur product, the capital cost of this process is substantially lower. Therefore, the process resilience will be higher 
if the reduced capital cost is invested in alternative resilience enhancement strategies, such as employing multiple parallel processes. Overall, the optimal process design selects technologies that enhance resilience by either directly generating products or by saving capital investment for other resilience enhancement strategies.

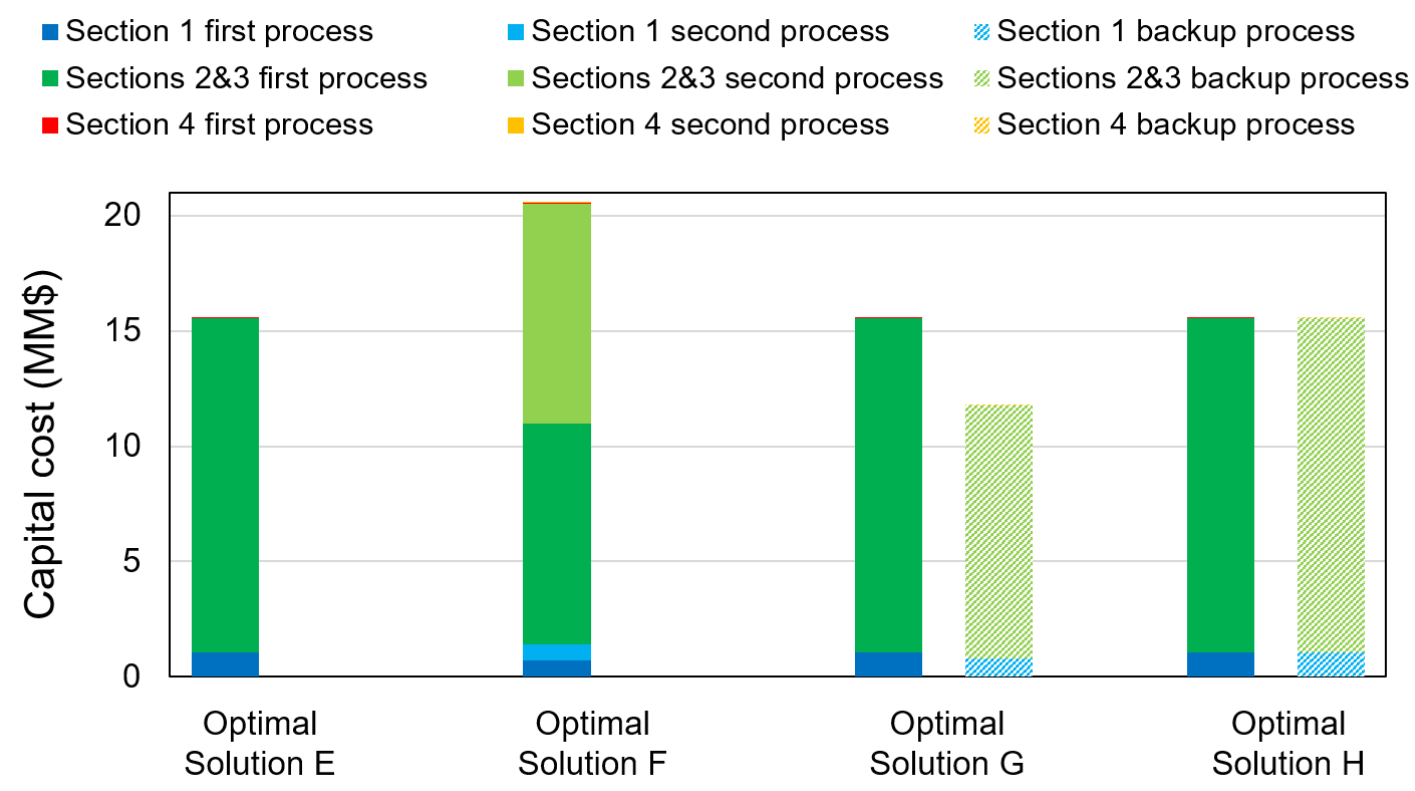

Figure 72. Capital cost breakdowns of the optimal solutions E, F, G, and H on the Paretooptimal curve in Figure 71.

The breakdowns of capital costs of the optimal solutions E, F, G, and $\mathrm{H}$ are presented in Figure 72. The integrated dehydration and NGLs recovery process contribute to over $90 \%$ of the total capital cost. It is noted that two resilience enhancement strategies are implemented. The optimal solution F employs two parallel operating processes for the integrated dehydration and NGLs recovery process, while backup processes are built for all the sections in the optimal 
solutions $\mathrm{G}$ and $\mathrm{H}$. The results also indicate that employing parallel processes can lead to a higher resilience with a low capital investment, but building backup processes is desirable if the capital investment is high.

(E)

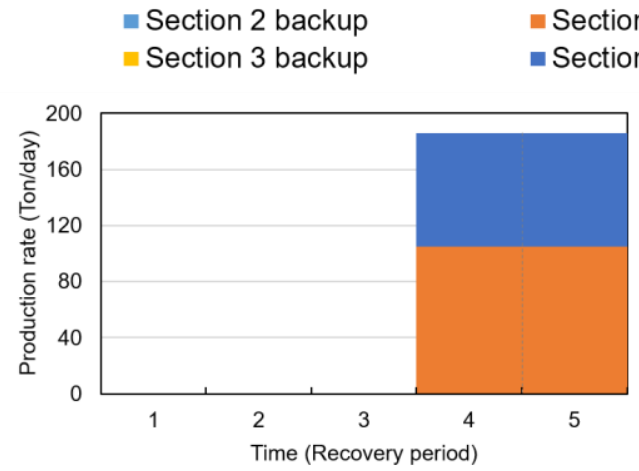

(F)

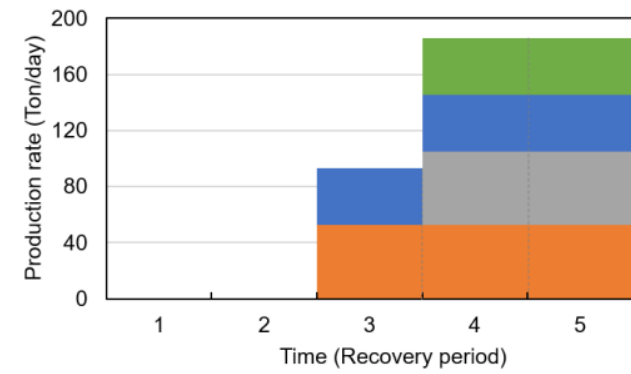

(G)
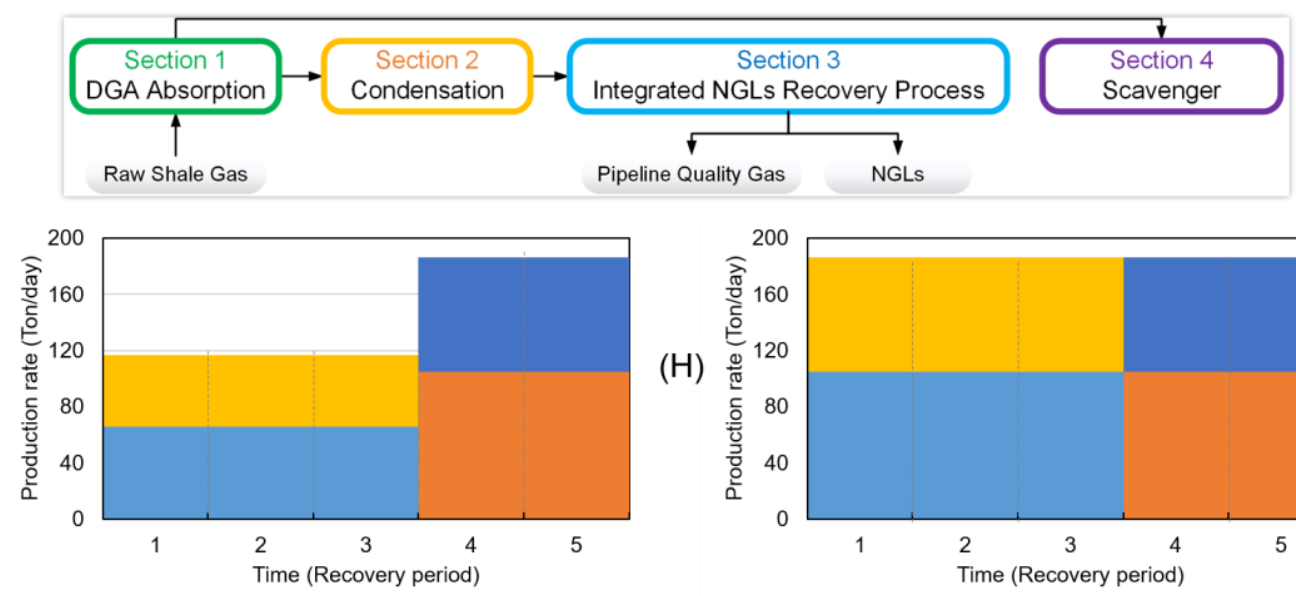

$(\mathrm{H})$

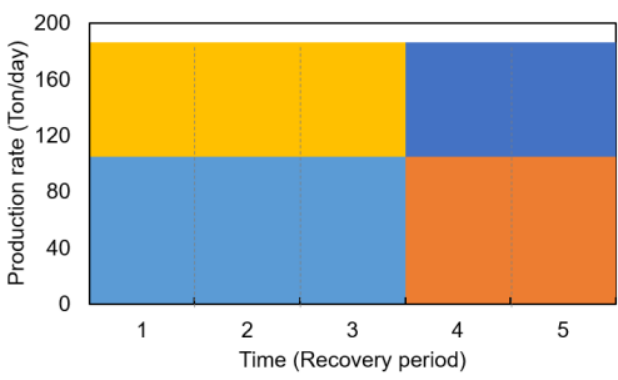

Figure 73. Worst-case realizations of the optimal solutions E, F, G, and H on the Paretooptimal curve in Figure 71. The selected technology/process alternatives in all the sections are shown in the middle.

The worst-case realizations of the optimal solutions $\mathrm{E}, \mathrm{F}, \mathrm{G}$, and $\mathrm{H}$ are shown in Figure 73. The worst-case realization of the optimal solution $\mathrm{F}$ is distinct from the others, because it employs two parallel operating processes in the dehydration section and the NGLs recovery section. As a process with a smaller capacity can be repaired and tested more efficiently, a half of the capacity of the operating 
processes is recovered in time period 3 . In time period 4 , the remaining operating processes are recovered, and the production rates in time periods 4 and 5 are the same as those in the optimal solution E. Overall, a higher capital investment enables more products to be manufactured in time period 3. When the capital investment keeps increasing, building backup processes can further reduce the loss of products after the occurrence of disruptive events as in the optimal solutions $\mathrm{G}$ and $\mathrm{H}$.

\subsection{Summary}

In this chapter, we addressed the resilient design and operations of process systems in response to disruptive events. We proposed a quantitative measure of resilience for process systems as a ratio of the quantity of products manufactured with disruptive events to that without disruptive events. Moreover, we introduced five resilience enhancement strategies for process systems, including selecting the most resilient technology/process alternatives, increasing the capacities of operating processes, employing parallel operating processes, building backup processes, and optimizing the operating levels after the occurrence of disruptive events. We further proposed a general framework for resilience optimization to incorporate the quantitative measure and the resilience enhancement strategies. The framework consisted of three steps, namely preliminary risk assessment, developing a multiobjective two-stage ARMIFP model, and solving the 
optimization problem with a tailored solution algorithm. The objective functions of the multiobjective two-stage ARMIFP model were to maximize the resilience under the worst-case realization of disruptive events and to minimize the total capital cost. The model determined the optimal network configuration, equipment capacities, and capital costs in the first stage, and the optimal number of available processes and operating levels in each time period in the second stage.

The applicability of the proposed resilience optimization framework was illustrated through the design and planning of a chemical process network and the superstructure optimization of shale gas processing and NGLs recovery processes. The maximum resilience of the chemical process network ranged from 0.71 to 1 if two out of six processes in the network failed after the occurrence of a disruptive event, while the corresponding minimum capital cost ranged from $\$ 4.32 \mathrm{MM}$ to \$8.64 MM. In the second application, the optimal process design selected DGA adsorption, condensation, integrated NGLs recovery process, and scavenger. The maximum resilience ranged from 0.4 to 1 , while the corresponding minimum capital cost ranged from $\$ 15.61 \mathrm{MM}$ to $\$ 31.22 \mathrm{MM}$. The tailored solution algorithm could efficiently solve the resulting multiobjective two-stage ARMIFP problems that could not be handled by any off-the-shelf optimization solvers directly. 
A practical limitation of the proposed framework is that OPEX has not been considered in the economic objective function of the proposed ARMIFP model. As OPEX involves the second-stage decisions, the economic objective function for both CAPEX and OPEX would consist of a three-level structure, which cannot be converted to a constraint as in the proposed tailored solution algorithm. It may involve more sophisticated multiobjective optimization method to handle two three-level objective functions.

\subsection{Appendix A: The conventional design and operations problem}

The conventional design and operations problem minimizes total capital cost without disruptive events.

$$
N O=\sum_{i \in I, j \in J}\left[\sum_{m \in M}\left(n u_{m} \cdot C C_{i, j, m}\right)+C C R_{i, j}\right]
$$

The objective function in this problem is provided in constrain (1.A1). We show the model formulation of the conventional design and operations problem as follows.

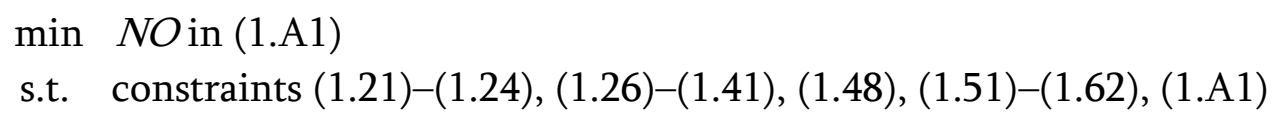

\subsection{Appendix B: The auxiliary parametric problem}

The auxiliary parametric problem $P(r)$ is a function of the auxiliary parameter $r$. The optimal solution of the orginal optimization problem is identical to the 
optimal solution of the auxiliary parametric problem with the parameter $r^{*}$ such that $P\left(r^{*}\right)=0$.

$$
\sum_{i \in I, j \in J}\left[\sum_{m \in M}\left(n u_{m} \cdot C C_{i, j, m}\right)+C C R_{i, j}\right] \leq t c c
$$

The objective function of minimizing the total capital cost is converted to constraint (1.B2). We present the formulation of auxiliary parametric problem as follows.

$$
P(r)=\max -r \cdot t t \cdot \sum_{i \in I, j \in J, m \in M, k \in K}\left(n u_{m} \cdot C A_{i, j, m, k}^{\text {product }}\right)+\min _{S I \in U} \max _{(Z, Z R, M) \in O} \sum_{i \in I, j \in J, k \in K, t \in T} M_{i, j, k, t}^{\text {product }}
$$

s.t. first-stage constraints (1.21)-(1.41), (1.B2)

Where $U=\left\{S I \in\{0,1\}^{|I| \times|D|}:\right.$ constraints (4) $\left.-(5)\right\}$,

$O=\{$ recovery constraints $(27)-(32)$; operating level constraints $(33)-(47)\}$.

\subsection{Appendix C: The master problem of the auxiliary parametric problem}

\subsection{1 (C1) Master problem}

The master problem is a relaxation of the auxiliary parametric problem. It includes all the first-stage constraints of the auxiliary parametric problem, and a set of modified second-stage constraints that are limited to a subset of the realizations of the uncertainty parameters.

$$
\begin{aligned}
& \eta \leq \sum_{i \in I, j \in J, k \in K, t \in T} M_{i, j, k, t, s}^{\text {product }}, \quad \forall s \in S \\
& Z_{i, j, m, t, s} \leq n u_{m} \cdot Y_{i, j, m}, \quad \forall i \in I, j \in J, m \in M, t \in T, s \in S
\end{aligned}
$$




$$
\begin{aligned}
& Z_{i, j, m, 1, s} \leq n u_{m} \cdot Y_{i, j, m} \cdot\left(1-s i i_{i, d, s}\right), \quad \forall i \in I, j \in J, m \in M, d \in D, s \in S \\
& Z_{i, j, m, t, s}=Z_{i, j, m, 1, s}, \quad \forall i \in I, j \in J, m \in M, t \in T B_{i, j, m}, s \in S \\
& Z_{i, j, m, t-1, s} \leq Z_{i, j, m, t, s}, \quad \forall i \in I, j \in J, m \in M, t \in T A_{i, j, m}, s \in S \\
& Z_{i, j, m, t, s} \leq Z_{i, j, m, t^{\prime}, s}+1, \quad \forall i \in I, j \in J, m \in M,\left(t, t^{\prime}\right) \in\left(T A_{i, j, m}, T L_{i, j, m, t}\right), s \in S \\
& Z R_{i, j, s} \leq \sum_{d \in D}\left(r i_{i, d} \cdot s i i_{i, d, s}\right), \quad \forall i \in I, j \in J, s \in S \\
& \sum_{k \in K} M_{i, j, k, t, s}^{i n} \leq \sum_{m \in M, k \in K}\left(n u_{m} \cdot C A_{i, j, m}\right), \quad \forall i \in I, j \in J, t \in T, s \in S \\
& \sum_{k \in K} M_{i, j, k, t, s}^{i n} \leq \sum_{m \in M, k \in K}\left(Z_{i, j, m, t, s} \cdot C A_{i, j, m}\right)+Z R_{i, j, s} \cdot C A R_{i, j}, \forall i, j, t, s \\
& \sum_{k \in K} M_{i, j, k, t, s}^{\text {product }} \leq \sum_{m \in M, k \in K}\left(n u_{m} \cdot C A_{i, j, m, k}^{\text {product }}\right), \quad \forall i \in I, j \in J, t \in T, s \in S \\
& M_{t, s}^{\text {feed }} \leq u b^{\text {feed }}, \quad \forall t \in T, s \in S \\
& \sum_{j \in J} M_{1, j, k, t, s}^{u p}=w f_{k}^{\text {feed }} \cdot M_{t, s}^{\text {feed }}, \quad \forall k \in K, t \in T, s \in S \\
& \sum_{j \in J} M_{i, j, k, t, s}^{u p}=\sum_{j \in J} M_{i-1, j, k, t, s}^{d o w n}, \quad \forall i \geq 2, k \in K, t \in T, s \in S \\
& \sum_{j \in J} M_{i, j, k, t, s}^{\text {reuse }}=\sum_{i^{\prime} \in R E_{i}, j \in J} M_{i^{\prime}, j, k, t, s}^{\text {recycle }}, \quad \forall i \in I, k \in K, t \in T, s \in S \\
& M_{i, j, k, t, s}^{u p}+M_{i, j, k, t, s}^{\text {reuse }}+M_{i, j, k, t, s}^{\text {makeup }}=\sum_{k^{\prime} \in K}\left[p_{i, j, k, k^{\prime}}^{\text {makeup }} \cdot\left(M_{i, j, k^{\prime}, t, s}^{u p}+M_{i, j, k^{\prime}, t, s}^{\text {reuse }}\right)\right], \\
& \forall i \in I, j \in J, k \in K, t \in T, s \in S \\
& M_{i, j, k, t, s}^{i n}=M_{i, j, k, t, s}^{u p}+M_{i, j, k, t, s}^{\text {reuse }}+M_{i, j, k, t, s}^{\text {makeup }}, \quad \forall i \in I, j \in J, k \in K, t \in T, s \in S \\
& M_{i, j, k, t, s}^{i n}+\sum_{k^{\prime} \in K}\left(s c_{i, j, k, k^{\prime}} \cdot M_{i, j, k^{\prime}, t, s}^{i n}\right)=M_{i, j, k, t, s}^{\text {out }}, \quad \forall i \in I, j \in J, k \in K, t \in T, s \in S \\
& M_{i, j, k, t, s}^{\text {out }}=M_{i, j, k, t, s}^{\text {down }}+M_{i, j, k, t, s}^{\text {recycle }}+M_{i, j, k, t, s}^{\text {product }}+M_{i, j, k, t, s}^{\text {waste }}, \quad \forall i, j, k, t, s \\
& M_{i, j, k, t, s}^{\text {down }}=s f_{i, j, k}^{\text {down }} \cdot M_{i, j, k, t, s}^{\text {out }}, \quad \forall i \in I, j \in J, k \in K, t \in T, s \in S
\end{aligned}
$$




$$
\begin{aligned}
& M_{i, j, k, t, s}^{\text {recycle }}=s f_{i, j, k}^{\text {recycle }} \cdot M_{i, j, k, t, s}^{\text {out }}, \quad \forall i \in I, j \in J, k \in K, t \in T, s \in S \\
& M_{i, j, k, t, s}^{\text {product }}=s f_{i, j, k}^{\text {product }} \cdot M_{i, j, k, t, s}^{\text {out }}, \quad \forall i \in I, j \in J, k \in K, t \in T, s \in S \\
& \sum_{i \in I, j \in J} M_{i, j, k, t_{n}, s}^{\text {product }} \geq p_{k}^{\text {demand }}, \quad \forall k \in K, s \in S \\
& M O=-r \cdot t t \cdot \sum_{i \in I, j \in J, m \in M, k \in K}\left(n u_{m} \cdot C A_{i, j, m, k}^{\text {product }}\right)+\eta
\end{aligned}
$$

The second-stage objective function of the auxiliary parametric problem is replaced by variable $\eta$, which is bounded above by the second-stage objective function values in all the identified realizations in constraint (1.C1). The objective function of the master problem is defined in constraint (1.C23). We show the formulation of the master problem as follows.

$\max M O$ in (1.C23)

s.t. $\quad$ constraints (1.21)-(1.41), (1.B2), (1.C1)-(1.C23)

\subsection{2 (C2) Linearization of bilinear terms}

As a set of bilinear terms $Z_{i, j, m, t, s^{*}} C A_{i, j, m}$ are included in constraint (1.C9), the master problem is an MINLP problem, which can be nontrivial to solve if a large number of realizations are added to the master problem. To solve the master problem efficiently, we exploit the properties of the bilinear terms and formulate an MILP master problem that has the same optimal solution and can be tackled by MILP solvers.

Although $Z_{i, j, m, t, s}$ and $Z R_{i, j, s}$ are defined as a continuous variable, the relevant constraints only enforce their integer upper and lower bounds. As the objective 
function of the master problem is maximized, $Z_{i, j, m, t, s}$ and $Z R_{i, j, s}$ should take the upper bound value as the optimal solution. This property is proved as follows.

Lemma. The optimal solutions of $Z_{i, j, m, t, s}$ and $Z R_{i, j, s}$ in the master problem are integers.

Proof. Let $G\left(M_{i, j, k, t, s}^{i n}\right)=M_{i, j, k, t, s}^{\text {product }}$ denote the linear relationship that maps the flow rate of the "in" flow to the "product" flow by constraints (1.C12)-(1.C21). $\forall i \in I, j \in J, m \in M, t \in T A_{i, j, m}, s \in S \quad, \quad \exists Z_{i, j, m, t, s}^{1} \leq Z_{i, j, m, t, s}^{2} \leq n u_{m} \cdot Y_{i, j, m}$ $Z R_{i, j, s}^{1} \leq Z R_{i, j, s}^{2} \leq \sum_{d \in D}\left(r i_{i, d} \cdot s i i_{i, d, s}\right) \quad, \quad\left(\eta^{1}, M_{i, j, k, t, s}^{\text {product }, 1}, M_{i, j, k, t, s}^{i n, 1}, Z_{i, j, m, t, s}^{1}, Z R_{i, j, s}^{1}\right) \quad$ and $\left(\eta^{2}, M_{i, j, k, t, s}^{p r o d u c t, 2}, M_{i, j, k, t, s}^{i n, 2}, Z_{i, j, m, t, s}^{2}, Z R_{i, j, s}^{2}\right)$ are two feasible solutions of the master problem, where the first-stage decisions $Y, C A$, and $C A R$ are fixed. We have $M_{i, j, k, t, s}^{i n, 1} \leq M_{i, j, k, t, s}^{i n, 2}$ from constraint (1.C9) and then based on the linear mass balance relationship, $M_{i, j, k, t, s}^{\text {product,1 }}=G\left(M_{i, j, k, t, s}^{i n, 1}\right) \leq G\left(M_{i, j, k, t, s}^{i n, 2}\right)=M_{i, j, k, t, s}^{\text {product }, 2}$. Next, from constraint (1.C1), we have $\eta^{1} \leq \eta^{2}$, which leads to the following relationship.

$$
\begin{aligned}
& M O^{1}=-r \cdot t t \cdot \sum_{i \in I, j \in J, m \in M, k \in K}\left(n u_{m} \cdot C A_{i, j, m, k}^{\text {product }}\right)+\eta^{1} \\
& \leq M O^{2}=-r \cdot t t \cdot \sum_{i \in I, j \in J, m \in M, k \in K}\left(n u_{m} \cdot C A_{i, j, m, k}^{\text {product }}\right)+\eta^{2}
\end{aligned}
$$

In order to maximize the objective function $M O, Z_{i, j, m, t, s}$ is pushed to its integer upper bound at $t \in T A_{i, j, m}$, and $Z R_{i, j, s}$ is pushed to its upper bound $\sum_{d \in D}\left(r i_{i, d} \cdot s i i_{i, d, s}\right)$. 
As $Z_{i, j, m, t, s}$ is an integer at $t \in T B_{i, j, m}$ by constraints (1.C3) and (1.C4), the optimal solutions of $Z_{i, j, m, t, s}$ and $Z R_{i, j, s}$ are integers.

$$
\begin{aligned}
& Z_{i, j, m, t, s}=\sum_{l \in L B_{i, j, m}} b v_{l} \cdot Z B_{i, j, m, t, s, l}, \quad \forall i \in I, j \in J, m \in M, t \in T, s \in S \\
& \sum_{l \in L B_{i, j, m}} Z B_{i, j, m, t, s, l} \leq 1, \quad \forall i \in I, j \in J, m \in M, t \in T, s \in S
\end{aligned}
$$

Therefore, the feasible region of $Z_{i, j, m, t, s}$ can be restricted to a set of integer values by constraints (1.C24) and (1.C25) without affecting the optimal objective function value. $b_{V l}$ denotes a possible integer value of $Z_{i, j, m, t, s}$ and $Z B_{i, j, m, t, s, l}$ is a binary variable.

$$
\begin{aligned}
& B T_{i, j, m, t, s, l} \leq C A_{i, j, m}, \quad \forall i \in I, j \in J, m \in M, t \in T, s \in S, l \in L B_{i, j, m} \\
& B T_{i, j, m, t, s, l} \leq \frac{u b_{i, j}^{i n} \cdot Z B_{i, j, m, t, s, l}}{n u_{m}}, \quad \forall i \in I, j \in J, m \in M, t \in T, s \in S, l \in L B_{i, j, m} \\
& B T_{i, j, m, t, s, l} \geq C A_{i, j, m}-\frac{u b_{i, j}^{i n}}{n u_{m}} \cdot\left(1-Z B_{i, j, m, t, s, l}\right), \quad \forall i, j, m, t, s, l \in L B_{i, j, m}
\end{aligned}
$$

The bilinear term $Z_{i, j, m, t, s^{*}} C A_{i, j, m}$ is then equal to the sum of multiple bilinear terms $b_{v /} \cdot Z B_{i, j, m, t, s, l} C A_{i, j, m}$. Each of the new bilinear terms can be equivalently substituted by a nonnegative variable $B T_{i, j, m, t, s, l}$ and a set of mixed-integer linear constraints (1.C26)-(1.C28) following the Glover's linearization scheme [217].

$$
\begin{aligned}
& Z R_{i, j, s}=Z R B_{i, j, s}, \quad \forall i \in I, j \in J, s \in S \\
& B T R_{i, j, s} \leq C A R_{i, j}, \quad \forall i \in I, j \in J, s \in S \\
& B T R_{i, j, s} \leq u b_{i, j}^{i n} \cdot Z R B_{i, j, s}, \quad \forall i \in I, j \in J, s \in S
\end{aligned}
$$




$$
B T R_{i, j, s} \geq C A R_{i, j}-u b_{i, j}^{i n} \cdot\left(1-Z R B_{i, j, s}\right), \quad \forall i \in I, j \in J, s \in S
$$

Similarly, continuous variable $Z R_{i, j, s}$ can be replaced by a binary variable $Z R B_{i, j, s}$ without affecting the optimal objective function value. Then, bilinear term $Z R B_{i, j, s} C A R_{i, j, m}$ can be substituted by a nonnegative variable $B T R_{i, j, s}$ and a set of mixed-integer linear constraints (1.C30)-(1.C32).

$$
\sum_{k \in K} M_{i, j, k, t, s}^{i n} \leq \sum_{m \in M, l \in L L_{i, j}}\left(b v_{l} \cdot B T_{i, j, m, t, s, l}\right)+B T R_{i, j, s}, \quad \forall i \in I, j \in J, t \in T, s \in S
$$

Finally, constraint (1.C9) is replaced by constraint (1.C33), and the master problem is reformulated as follows.

$\max \quad M O$ in (1.C23)

s.t. $\quad$ constraints (1.21)-(1.41), (1.B2), (1.C1)-(1.C8), (1.C10)-(1.C33)

\subsection{Appendix D: The subproblem of the auxiliary problem}

\subsection{1 (D1) Subproblem}

The goal of the subproblem is to determine the optimal second-stage decisions with fixed first-stage decisions. However, the second-stage min-max problem cannot be handled directly by any off-the-shelf optimization solvers. To tackle this computational challenge, the inner maximization problem is dualized and incorporated into the outer minimization problem.

$$
P 1_{i, j, m, 1}+\sum_{d \in D} P 2_{i, j, m, d}-\sum_{t \in T B_{i, j, m}} P 3_{i, j, m, t}-P 8_{i, j, 1} \cdot c a i_{i, j, m} \geq 0, \quad \forall i \in I, j \in J, m \in M
$$




$$
\begin{aligned}
& P 1_{i, j, m, t}+P 3_{i, j, m, t}-P 8_{i, j, t} \cdot c a i_{i, j, m} \geq 0 \text {, } \\
& \forall i \in I, j \in J, m \in M, t \in T B_{i, j, m}, t \notin T L T_{i, j, m}, t+1 \notin T A_{i, j, m} \\
& P 1_{i, j, m, t}+P 3_{i, j, m, t}-\sum_{t^{\prime} \in T L I_{i, j, m, t}} P 5_{i, j, m, t^{\prime}}-P 8_{i, j, t} \cdot c a i_{i, j, m} \geq 0, \\
& \forall i \in I, j \in J, m \in M, t \in T B_{i, j, m}, t \in T L T_{i, j, m}, t+1 \notin T A_{i, j, m} \\
& P 1_{i, j, m, t}+P 3_{i, j, m, t}+P 4_{i, j, m, t+1}-\sum_{t^{\prime} \in T L I_{i, j, m, t}} P 5_{i, j, m, t^{\prime}}-P 8_{i, j, t} \cdot c a i_{i, j, m} \geq 0 \\
& \forall i \in I, j \in J, m \in M, t \in T B_{i, j, m}, t \in T L T_{i, j, m}, t+1 \in T A_{i, j, m} \\
& P 1_{i, j, m, t}-P 4_{i, j, m, t}+P 4_{i, j, m, t+1}+P 5_{i, j, m, t}-\sum_{t^{\prime} \in T L I_{i, j m, t}} P 5_{i, j, m, t^{\prime}}-P 8_{i, j, t} \cdot c a i_{i, j, m} \geq 0, \\
& \forall i \in I, j \in J, m \in M, t \in T A_{i, j, m} \\
& P 6_{i, j}-\sum_{i \in T}\left(P 8_{i, j, t} \cdot \operatorname{cari}_{i, j}\right) \geq 0, \quad \forall i \in I, j \in J \\
& P 10_{t}-\sum_{k \in K}\left(P 11_{k, t} \cdot w f_{k}^{\text {feed }}\right) \geq 0, \quad \forall t \in T \\
& P 11_{k, t}+P 14_{1, j, k, t}-\sum_{k^{\prime} \in K}\left(P 14_{1, j, k^{\prime}, t} \cdot p_{1, j, k^{\prime}, k}^{\text {makeup }}\right)-P 15_{i, j, k, t} \geq 0, \quad \forall j \in J, k \in K, t \in T \\
& P 12_{i, k, t}+P 14_{i, j, k, t}-\sum_{k^{\prime} \in K}\left(P 14_{i, j, k^{\prime}, t} \cdot p_{i, j, k^{\prime}, k}^{\text {makeup }}\right)-P 15_{i, j, k, t} \geq 0, \forall i \geq 2, j, k, t \\
& P 13_{i, k, t}+P 14_{i, j, k, t}-\sum_{k^{\prime} \in K}\left(P 14_{i, j, k^{\prime}, t} \cdot p_{i, j, k^{\prime}, k}^{\text {makeup }}\right)-P 15_{i, j, k, t} \geq 0, \forall i, j, k, t \\
& P 14_{i, j, k, t}-P 15_{i, j, k, t} \geq 0, \quad \forall i \in I, j \in J, k \in K, t \in T \\
& P 7_{i, j, t}+P 8_{i, j, t}+P 15_{i, j, k, t}-P 16_{i, j, k, t}-\sum_{k^{\prime} \in K}\left(P 16_{i, j, k^{\prime}, t} \cdot s c_{i, j, k^{\prime}, k}\right) \geq 0, \forall i, j, k, t \\
& -P 16_{i, j, k, t}+P 17_{i, j, k, t}-P 18_{i, j, k, t} \cdot s f_{i, j, k}^{\text {down }}-P 19_{i, j, k, t} \cdot s f_{i, j, k}^{\text {recycle }} \\
& -P 2 O_{i, j, k, t} \cdot s f_{i, j, k}^{\text {product }} \geq 0, \quad \forall i \in I, j \in J, k \in K, t \in T \\
& -P 12_{i, k, t+1}-P 17_{i, j, k, t}+P 18_{i, j, k, t} \geq 0, \quad \forall i \in I, j \in J, k \in K, t \in T \\
& -\sum_{R E I_{i}} P 13_{i^{\prime}, k, t}-P 17_{i, j, k, t}+P 19_{i, j, k, t} \geq 0, \quad \forall i \in I, j \in J, k \in K, t \in T
\end{aligned}
$$




$$
\begin{aligned}
& P 9_{i, j, t}-P 17_{i, j, k, t}+P 2 O_{i, j, k, t} \geq 1, \quad \forall i \in I, j \in J, k \in K, t<t_{n} \\
& P 9_{i, j, t_{n}}-P 17_{i, j, k, t_{n}}+P 2 O_{i, j, k, t_{n}}-P 21_{k} \geq 1, \quad \forall i \in I, j \in J, k \in K \\
& -P 17_{i, j, k, t} \geq 0, \quad \forall i \in I, j \in J, k \in K, t \in T \\
& S O=\sum_{i \in I, j \in J, m \in M, t \in T}\left(P 1_{i, j, m, t} \cdot n u_{m} \cdot y i_{i, j, m}\right) \\
& \quad+\sum_{i \in I, j \in J, m \in M, d \in D}\left[P 2_{i, j, m, d} \cdot n u_{m} \cdot y i_{i, j, m} \cdot\left(1-S I_{i, d}\right)\right] \\
& \quad+\sum_{i \in I, j \in J, m \in M, t \in T A_{i, j, m}} P 5_{i, j, m, t}+\sum_{i \in I, j \in J, d \in D}\left(P 6_{i, j} \cdot r i_{i, d} \cdot S I_{i, d}\right) \\
& \quad+\sum_{i \in I, j \in J, m \in M, t \in T}\left(P 7_{i, j, t} \cdot n u_{m} \cdot c a i_{i, j, m}\right) \\
& \quad+\sum_{i \in l, j \in J, m \in M, k \in K, t \in T}\left(P 9_{i, j, t} \cdot n u_{m} \cdot c a i_{i, j, m, k}^{p r o d u c t}\right) \\
& \quad+\sum_{t \in T}\left(P 10_{t} \cdot u b^{\text {feed }}\right)-\sum_{k \in K}\left(P 21_{k} \cdot p_{k}^{\text {demand }}\right)
\end{aligned}
$$

The formulation of the subproblem is presented as follows.

$$
\begin{array}{cl}
\min & S O \text { in (1.D19) } \\
\text { s.t. } & \text { constraints (1.19)-(1.20), (1.D1)-(1.D19) }
\end{array}
$$

\subsection{2 (D2) Linearization of bilinear terms}

Since bilinear terms $P 2_{i, j, m, d} \cdot\left(1-S I_{i, d}\right)$ and $P 6_{i, j} S I_{i, d}$ are included in the objective function $S O$ in constraint (1.D19), the resulting subproblem must be solved by MINLP solvers. Given that $S I_{i, d}$ is a binary variable, we replace the bilinear terms by $B T 1_{i, j, m, d}$ and $B T 2_{i, j, d}$, respectively, and add constraints (1.D20)-(1.D25) following the Glover's linearization scheme [217]. Moreover, the objective function is reformulated by constraint (1.D26).

$$
B T 1_{i, j, m, d} \leq P 2_{i, j, m, d}, \quad \forall i \in I, j \in J, m \in M, d \in D
$$




$$
\begin{aligned}
& B T 1_{i, j, m, d} \leq u b b t 1 \cdot\left(1-S I_{i, d}\right), \quad \forall i \in I, j \in J, m \in M, d \in D \\
& B T 1_{i, j, m, d} \geq P 2_{i, j, m, d}-u b b t 1 \cdot S I_{i, d}, \quad \forall i \in I, j \in J, m \in M, d \in D \\
& B T 2_{i, j, d} \leq P 6_{i, j}, \quad \forall i \in I, j \in J, d \in D \\
& B T 2_{i, j, d} \leq u b b t 2 \cdot S I_{i, d}, \quad \forall i \in I, j \in J, d \in D \\
& B T 2_{i, j, d} \geq P 6_{i, j}-u b b t 2 \cdot\left(1-S I_{i, d}\right), \quad \forall i \in I, j \in J, d \in D \\
& S O=\sum_{i \in I, j \in J, m \in M, t \in T}\left(P 1_{i, j, m, t} \cdot n u_{m} \cdot y i_{i, j, m}\right) \\
& +\sum_{i \in I, j \in J, m \in M, d \in D}\left(B T 1_{i, j, m, d} \cdot n u_{m} \cdot y i_{i, j, m}\right) \\
& +\sum_{i \in I, j \in J, m \in M, t \in T A_{i, j, m}} P 5_{i, j, m, t}+\sum_{i \in I, j \in J, d \in D}\left(B T 2_{i, j, d} \cdot r i_{i, d}\right) \\
& +\sum_{i \in I, j \in J, m \in M, t \in T}\left(P 7_{i, j, t} \cdot n u_{m} \cdot c a i_{i, j, m}\right) \\
& +\sum_{i \in I, j \in J, m \in M, k \in K, t \in T}\left(P 9_{i, j, t} \cdot n u_{m} \cdot c a i_{i, j, m, k}^{p r o d u c t}\right) \\
& +
\end{aligned}
$$

The reformulated subproblem is presented as follows and can be handled by MILP solvers.

$\min S O$ in (1.D26)

s.t. constraints (1.19)-(1.20), (1.D1)-(1.D18), (1.D20)-(1.D26)

\subsection{Appendix E: Parameters in the recovery constraints in the applications}

In both applications, a total of five time intervals are considered, or $T=\{1,2,3,4,5\}$.

By enforcing $M=\{1,2\}$, at most two parallel units are allowed for each process. If $m=1$, one process with a relatively larger capacity would be built and each 
process/section would need two time intervals to recover from disruption. Therefore, the availability of a process in $t=2$ and $t=3$ would be the same with that in $t=1$, or $T B_{i, j, 1}=\{2,3\}$. The availability in the following time intervals or $T A_{i, j, 1}=\{4,5\}$ can stay the same with that two time intervals before, or it would become available given the recovery effort. That said, $T L_{i, j, 1,4}=\{2\}$ and $T L_{i, j, 1,5}=\{3\}$. If $m=2$, two parallel units would be built for each process and each process/section would need one time interval to recover from disruption. The corresponding subsets are given as follows: $T B_{i, j, 2}=\{2\}$, $T A_{i, j, 2}=\{3,4,5\}, T L_{i, j, 2,3}=\{2\}, T L_{i, j, 2,4}=\{3\}, T L_{i, j, 2,5}=\{4\}$. 


\section{CHAPTER 7}

\section{CONCLUSIONS}

Two energy systems, namely the algal biofuel and bioproduct manufacturing system and the shale gas processing and chemical manufacturing system, have been studied in this chapter. We believe that the research presented in this dissertation lays a foundation for designing the algal biofuel and bioproduct manufacturing system and the shale gas processing and chemical manufacturing system. Moreover, the proposed modeling frameworks and optimization algorithms can be readily employed in the sustainable design and operations of other energy systems. The summary of the dissertation and future work are provided in this section.

\subsection{Summary of the contributions}

We proposed by far the most comprehensive superstructure for algal biofuel and bioproduct manufacturing processes. A key contribution of the proposed superstructure was the bioproduct manufacturing production processes to utilize the byproducts in biofuel production processes and generate carbon dioxide feed for microalgae growth. We developed a multiobjective mixed-integer fractional programming problem to optimize the functional unit based economic and environmental performance simultaneously. To effectively solve the proposed optimization problem, we developed a tailored global optimization algorithm to integrate the inexact parametric algorithm for the fractional objective function and branch-and-refine algorithm for the separable concave terms. We found that the environmental impacts of the resulting bioproducts were notably lower than those in 
the literature. The computational times by the proposed global optimization algorithm were much shorter than those by global optimization solvers.

We developed a two-stage adaptive robust optimization model to handle market related uncertainty in energy system design problems. Unlike conventional robust optimization models, the decisions in the proposed model were made in two stages according to whether they could be determined before or after uncertainty was realized. The objective function of the proposed optimization model was to maximize the return on investment, which was defined as a fraction term. Due to the multilevel structure and the fractional objective function, the problem could not be solved by any off-the-shelf optimization solvers or by existing solution methods. We proposed a tailored optimization algorithm to integrate the inexact parametric algorithm for the fractional objective function and the column-and-constraint generation algorithm for handling the multilevel optimization problems. The obtained optimal solution could effective hedge against the worst realization of uncertainty and the proposed optimization algorithm was found to efficiently return the optimal solutions.

We developed a novel consequential life cycle optimization (LCO) framework that simultaneously optimizes consequential environmental impacts and economic performance. We proposed a general system boundary for consequential LCA and a multiobjective optimization model by integrating process models, partial equilibrium market models with the tenets of consequential LCA and technoeconomic analysis methodologies. In the application to algal renewable diesel production processes, we performed thorough market analyses to identify 
consequences and found that the environmental impacts of the optimal process designs based on the proposed consequential LCO framework are significantly lower than those based on the existing attributional LCO framework.

We developed a novel process synthesis framework that combined product distribution optimization of chemical reactions and superstructure optimization of process flowsheets. The framework consisted of four steps. The product distributions of critical chemical reactions in a superstructure were first optimized to maximize the profits of the effluent products. In the second step, extensive process simulations were performed based on the optimal product distributions to collect high-fidelity process data. Next, an MINLP model was developed for superstructure optimization. In the last step, the optimal process design was validated by a whole-process simulation. The proposed framework was illustrated by an application to an integrated shale gas processing and chemical manufacturing process. The proposed superstructure consisted of 51,840 alternative process designs. We optimized the product distributions of steam cracking and developed a set of simulation models for all the involved processes in the superstructure. A multiobjective MINLP model was then formulated to address the sustainable design and synthesis of the integrated shale gas processing and chemical manufacturing process. The maximum net present value per GJ of raw shale gas was higher when the optimal product distributions of steam cracking were used, 
while the minimum globla warming potential and water footprint per GJ of raw shale gas were lower when the product distributions of steam cracking taken from the literature were used.

We proposed a novel quantitative measure of resilience for process systems as a ratio of the quantity of products manufactured with disruptive events to that without disruptive events. To improve system performance in face of disruptive events, we introduced five resilience enhancement strategies. Moreover, we proposed a general framework for resilience optimization to incorporate the quantitative measure and the resilience enhancement strategies. The framework consisted of three steps, namely preliminary risk assessment, developing a multiobjective two-stage ARMIFP model, and solving the optimization problem with a tailored solution algorithm. The objective functions of the multiobjective two-stage ARMIFP model were to maximize the resilience under the worst-case realization of disruptive events and to minimize the total capital cost. The model determined the optimal network configuration, equipment capacities, and capital costs in the first stage, and the optimal number of available processes and operating levels in each time period in the second stage. The applications showed that the optimal process design could effectively enhance the performance after the occurrence of disruptive events at the expense of higher capital expenditures. 


\subsection{Future work}

The general consequential LCO framework is first of its kind to integrate the concept of consequential LCA with optimization techniques. For practical purposes, a major assumption of the optimization model is to limit the system boundary within two levels of markets, resulting in underestimated environmental consequences. As we discussed in Chapter 4, there are several systematic tools available to evaluate consequential environmental impacts in the entire economy, such as computable general equilibrium models (CGE). Despite more markets included, these systematic tools do not differentiate products in the same industry and rely on relatively low-quality and outdated data. A future direction of consequential LCO is to improve the coverage of environmental consequences by leveraging the existing tools. As has been developed in attributional LCO [239], a hybrid LCO approach could make the most of the detailed but boundary-limited process-based LCO, and the broad but low-resolution input-output LCO. Similarly, we may integrate the proposed tailored market analysis for currently considered markets, and CGE models for other markets in the economy. There may be computational challenges due to the use of nonlinear functions in the CGE models and more sophisticated computational algorithms should be developed to efficiently solve the resulting optimization problems. 
We develop a holistic framework that combines product distribution optimization of chemical reactions and superstructure optimization of the process flowsheet. The two optimization tasks are conducted in a sequential manner, because simultaneous product distribution optimization and superstructure optimization can be computationally challenging. The future direction of combined superstructure optimization and product distribution optimization is to develop solution algorithms that can handle the resulting mixed-integer nonlinear dynamic optimization model efficiently. A promising strategy is to treat the product distribution optimization as a black box and approximate the mass and energy relationships with less computationally demanding surrogate models. It requires further efforts to balance the complexity and computational difficulty of the surrogate models.

The framework on resilient process design and operations of energy systems provides a new perspective into energy systems. There are a few directions to improve the optimization model so that it is able to capture more details of a process system after the occurrence of disruptive events. (1) The first is to include operating expenditures (OPEX) in the economic objective function. In the proposed model, the economic objective function considers only capital expenditures (CAPEX), because both CAPEX and resilience reflect the intrinsic properties of a process system. However, OPEX and CAPEX are an integral part in 
evaluating the economic performance of a process system. If OPEX is included in the objective function, it would be determined as the second-stage decisions in the third-level optimization problem. As a result, the economic objective function consists of a three-level structure that is the same with the resilience objective function. The resulting optimization model must be handled by tailored multiobjective optimization tools. (2) The second direction is to introduce externalities in the second-stage recovery model. Recovery resources are usually limited after the occurrence of disruptive events because professionals and relevant materials are expected to become demanding in the market. Recovery at the full speed may be too costly or even infeasible. Moreover, the disruptive events may also cause failures in downstream processes, so that the product demand may recover gradually. Therefore, it is sometimes unnecessary for the process system to recover at the full speed. Since it requires integer variables to account for externalities in the second-stage recovery model, more sophisticated solution algorithm should be proposed to handle the resulting two-stage adaptive robust optimization problem with integer recourse decisions. 


\section{REFERENCES}

[1] J. J. Klemes, E. N. Pistikopoulos, M. C. Georgiadis, and H. Lund, "Energy systems engineering," Energy, vol. 44, pp. 2-5, 2012.

[2] P. Liu, M. C. Georgiadis, and E. N. Pistikopoulos, "Advances in Energy Systems Engineering," Industrial \& Engineering Chemistry Research, vol. 50, pp. 4915-4926, 2011.

[3] EIA, "International Energy Outlook 2017," U.S. Department of Energy, Washington, DC, 2017.

[4] IPCC, Climate Change 2014, Mitigation of Climate Change. Contribution of Working Group III to the Fifth Assessment Report of the Intergovernmental Panel on Climate Change: Cambridge University Press, 2014.

[5] EPA. (2017). Renewable Fuel Standard Program. Available: https://www.epa.gov/renewable-fuel-standard-program

[6] U. Congress, "Energy independence and security act of 2007," Public Law, p. 2, 2007.

[7] Algal Biofuels and Federal Policy: RFS2 and Other Policy Issues. Available: http://www.wsgr.com/publications/PDFSearch/braff-042512.pdf

[8] B. R. Bakshi, "Methods and tools for sustainable process design," Curr. Opin. Chem. Eng., vol. 6, pp. 69-74, 2014.

[9] J. Gong and F. You, "Sustainable design and synthesis of energy systems," Curr. Opin. Chem. Eng., vol. 10, pp. 77-86, 2015.

[10] B. R. Bakshi and J. Fiksel, "The quest for sustainability: Challenges for process systems engineering," AIChE J., vol. 49, pp. 1350-1358, 2003.

[11] M. A. Duran and I. E. Grossmann, "Simultaneous-Optimization and Heat Integration of Chemical Processes," AIChE J., vol. 32, pp. 123-138, 1986.

[12] F. You and B. Wang, "Life Cycle Optimization of Biomass-to-Liquid Supply Chains with Distributed-Centralized Processing Networks," Ind. Eng. Chem. Res., vol. 50, pp. 10102-10127, 2011.

[13] M. Gassner and F. Marechal, "Methodology for the optimal thermoeconomic, multi-objective design of thermochemical fuel production from biomass," Computers \& Chemical Engineering, vol. 33, pp. 769-781, 2009.

[14] B. Wang, B. H. Gebreslassie, and F. You, "Sustainable design and synthesis of hydrocarbon biorefinery via gasification pathway: Integrated life cycle assessment and technoeconomic analysis with multiobjective superstructure optimization," Comput. Chem. Eng., vol. 52, pp. 55-76, 2013.

[15] B. H. Gebreslassie, M. Slivinsky, B. L. Wang, and F. Q. You, "Life cycle optimization for sustainable design and operations of hydrocarbon 
biorefinery via fast pyrolysis, hydrotreating and hydrocracking," Computers \& Chemical Engineering, vol. 50, pp. 71-91, 2013.

[16] Q. Zhang, J. Gong, M. Skwarczek, D. Yue, and F. You, "Sustainable Process Design and Synthesis of Hydrocarbon Biorefinery through Fast Pyrolysis and Hydroprocessing," AIChE J., vol. 60, pp. 980-994, 2014.

[17] V. Pham and M. El-Halwagi, "Process synthesis and optimization of biorefinery configurations," AIChE J., vol. 58, pp. 1212-1221, 2012.

[18] J. M. Ponce-Ortega, V. Pham, M. M. El-Halwagi, and A. A. El-Baz, "A Disjunctive Programming Formulation for the Optimal Design of Biorefinery Configurations," Ind. Eng. Chem. Res., vol. 51, pp. 3381-3400, 2012.

[19] B. H. Gebreslassie, R. Waymire, and F. You, "Sustainable design and synthesis of algae-based biorefinery for simultaneous hydrocarbon biofuel production and carbon sequestration," AIChE J., vol. 59, pp. 1599-1621, 2013.

[20] J. Gong and F. You, "Optimal Design and Synthesis of Algal Biorefinery Processes for Biological Carbon Sequestration and Utilization with Zero Direct Greenhouse Gas Emissions: MINLP Model and Global Optimization Algorithm," Ind. Eng. Chem. Res., vol. 53, pp. 1563-1579, 2014.

[21] J. Gong and F. You, "Global Optimization for Sustainable Design and Synthesis of Algae Processing Network for CO2 Mitigation and Biofuel Production Using Life Cycle Optimization," AIChE J., vol. 60, pp. 31953210, 2014.

[22] J. Gong and F. You, "Value-Added Chemicals from Microalgae: Greener, More Economical, or Both?," ACS Sustain. Chem. Eng., vol. 3, pp. 82-96, 2015.

[23] P. Liu, E. N. Pistikopoulos, and Z. Li, "A Multi-Objective Optimization Approach to Polygeneration Energy Systems Design," AIChE, vol. 56, pp. 1218-1234, 2010.

[24] C. He, F. Q. You, and X. Feng, "A Novel Hybrid Feedstock to Liquids and Electricity Process: Process Modeling and Exergoeconomic Life Cycle Optimization," AIChE, vol. 60, pp. 3739-3753, 2014.

[25] Y. Chen, T. A. Adams, and P. I. Barton, "Optimal Design and Operation of Flexible Energy Polygeneration Systems," Industrial \& Engineering Chemistry Research, vol. 50, pp. 4553-4566, 2011.

[26] C. He and F. You, "Toward more cost-effective and greener chemicals production from shale gas by integrating with bioethanol dehydration: Novel process design and simulation-based optimization," AIChE J., vol. 61, pp. 1209-1232, 2015. 
[27] C. He and F. You, "Shale Gas Processing Integrated with Ethylene Production: Novel Process Designs, Exergy Analysis, and Techno-Economic Analysis," Ind. Eng. Chem. Res., vol. 53, pp. 11442-11459, 2014.

[28] K. J. Gabriel, M. Noureldin, M. M. El-Halwagi, P. Linke, A. JiménezGutiérrez, and D. Y. Martínez, "Gas-to-liquid (GTL) technology: Targets for process design and water-energy nexus," Curr. Opin. Chem. Eng., vol. 5, pp. 49-54, 2014.

[29] D. J. Garcia and F. You, "Multiobjective optimization of product and process networks: General modeling framework, efficient global optimization algorithm, and case studies on bioconversion," AIChE J., vol. 61, pp. 530554, 2015.

[30] R. Lal, "Carbon sequestration," Philos T R Soc B, vol. 363, pp. 815-830, 2008.

[31] J. Ferrell and V. Sarisky-Reed, "National algal biofuels technology roadmap," US Department of Energy College Park, MD, 2010.

[32] T. Werpy, G. Petersen, A. Aden, J. Bozell, J. Holladay, J. White, et al., "Top value added chemicals from biomass. Volume 1-Results of screening for potential candidates from sugars and synthesis gas," DTIC Document, 2004.

[33] M. Pagliaro, R. Ciriminna, H. Kimura, M. Rossi, and C. Della Pina, "From glycerol to value-added products," Angew Chem Int Edit, vol. 46, pp. 44344440, 2007.

[34] C. H. Zhou, H. Zhao, D. S. Tong, L. M. Wu, and W. H. Yu, "Recent Advances in Catalytic Conversion of Glycerol," Catal Rev, vol. 55, pp. 369453, 2013.

[35] W. Bollmeier, S. Sprague, and F. No, "Aquatic Species Program," Solar Energy Research Inst., Golden, CO (USA), 1989.

[36] "Renewable Diesel from Algal Lipids: An Integrated Baseline for Cost, Emissions, and Resource Potential from a Harmonized Model," Argonne National Laboratory, National Renewable Energy Laboratory, Pacific Northwest National Laboratory, Argonne, IL, Golden, CO, and Richland, WA, U.S.A ANL/ESD/12-4, NREL/TP-5100-55431, PNNL-21437.

[37] M. Martin and I. E. Grossmann, "Simultaneous Optimization and Heat Integration for the Coproduction of Diesel Substitutes: Biodiesel (FAME and FAEE) and Glycerol Ethers from Algae Oil," Industrial \& Engineering Chemistry Research, vol. 53, pp. 11371-11383, 2014.

[38] M. Rizwan, J. H. Lee, and R. Gani, "Optimal processing pathway for the production of biodiesel from microalgal biomass: A superstructure based approach," Comput. Chem. Eng., vol. 58, pp. 305-314, 2013.

[39] A. Quaglia, B. Sarup, G. Sin, and R. Gani, "Integrated business and engineering framework for synthesis and design of enterprise-wide processing networks," Comput. Chem. Eng., vol. 38, pp. 213-223, 2012. 
[40] C. G. Gutierrez-Arriaga, M. Serna-Gonzalez, J. M. Ponce-Ortega, and M. M. El-Halwagi, "Sustainable Integration of Algal Biodiesel Production with Steam Electric Power Plants for Greenhouse Gas Mitigation," ACS Sustain. Chem. Eng., vol. 2, pp. 1388-1403, 2014.

[41] C. Silva, E. Soliman, G. Cameron, L. A. Fabiano, W. D. Seider, E. H. Dunlop, et al., "Commercial-Scale Biodiesel Production from Algae," Ind. Eng. Chem. Res., vol. 53, pp. 5311-5324, 2014.

[42] G. Pokoo-Aikins, A. Nadim, M. M. El-Halwagi, and V. Mahalec, "Design and analysis of biodiesel production from algae grown through carbon sequestration," Clean Technol Envir, vol. 12, pp. 239-254, 2010.

[43] Y. Chen, X. Li, T. A. Adams, and P. I. Barton, "Decomposition strategy for the global optimization of flexible energy polygeneration systems," AIChE, vol. 58, pp. 3080-3095, 2012.

[44] R. C. Baliban, J. A. Elia, C. A. Floudas, X. Xiao, Z. J. Zhang, J. Li, et al., "Thermochemical Conversion of Duckweed Biomass to Gasoline, Diesel, and Jet Fuel: Process Synthesis and Global Optimization," Industrial \& Engineering Chemistry Research, vol. 52, pp. 11436-11450, 2013.

[45] L. F. Lira-Barragán, J. M. Ponce-Ortega, M. Serna-González, and M. M. ElHalwagi, "Optimal design of process energy systems integrating sustainable considerations," Energy,

DOI: http://dx.doi.org/10.1016/j.energy.2014.04.111.

[46] W. D. Seider, J. D. Seader, and D. R. Lewin, Product \& process design principles: synthesis, analysis and evaluation, Hoboken, NJ, U.S.A.: John Wiley \& Sons, 2009.

[47] R. Davis, A. Aden, and P. T. Pienkos, "Techno-economic analysis of autotrophic microalgae for fuel production," Appl. Energy, vol. 88, pp. 3524-3531, 2011.

[48] A. O. Alabi, E. Bibeau, M. Tampier, and B. C. I. Council, Microalgae Technologies \& Processes for Biofuels-bioenergy Production in British Columbia: Current Technology, Suitability \& Barriers to Implementation: Final Report. British Columbia Innovation Council, 2009.

[49] M. Martín and I. E. Grossmann, "Optimal engineered algae composition for the integrated simultaneous production of bioethanol and biodiesel," AIChE J, vol. 59, pp. 2872-2883, 2013.

[50] E. H. Dunlop, A. K. Coaldrake, C. S. Silva, and W. D. Seider, "An Energylimited Model of Algal Biofuel Production: Toward the Next Generation of Advanced Biofuels," AIChE J., vol. 59, pp. 4641-4654, 2013.

[51] D. Bertsimas, D. B. Brown, and C. Caramanis, "Theory and Applications of Robust Optimization," SIAM Re., vol. 53, pp. 464-501, 2011. 
[52] D. J. Garcia and F. You, "Supply chain design and optimization: Challenges and opportunities," Comput. Chem. Eng., vol. 81, pp. 153-170, 2015.

[53] A. Ben-Tal, L. El Ghaoui, and A. Nemirovski, Robust optimization, Princeton, NJ, USA: Princeton University Press, 2009.

[54] V. Gabrel, C. Murat, and A. Thiele, "Recent advances in robust optimization: An overview," Eur. J. Oper. Res., vol. 235, pp. 471-483, 2014.

[55] P. Kouvelis and G. Yu, Robust discrete optimization and its applications: Springer Science \& Business Media, 2013.

[56] H. Shi and F. You, "A computational framework and solution algorithms for two-stage adaptive robust scheduling of batch manufacturing processes under uncertainty," AIChE J., vol. 62, pp. 687-703, 2016.

[57] G. Finnveden, M. Z. Hauschild, T. Ekvall, J. Guinee, R. Heijungs, S. Hellweg, et al., "Recent developments in Life Cycle Assessment," J. Environ. Manage., vol. 91, pp. 1-21, 2009.

[58] J. B. Guinée, R. Heijungs, G. Huppes, A. Zamagni, P. Masoni, R. Buonamici, et al., "Life Cycle Assessment: Past, Present, and Future†," Environ. Sci. Technol., vol. 45, pp. 90-96, 2010.

[59] A. Azapagic, "Life cycle assessment and its application to process selection, design and optimisation," Chem. Eng. J., vol. 73, pp. 1-21, 1999.

[60] A. Azapagic and R. Clift, "The application of life cycle assessment to process optimisation," Comput. Chem. Eng., vol. 23, pp. 1509-1526, 1999.

[61] J. B. Guinée, R. Heijungs, G. Huppes, A. Zamagni, P. Masoni, R. Buonamici, et al., "Life Cycle Assessment: Past, Present, and Future," Environ. Sci. Technol., vol. 45, pp. 90-96, 2011.

[62] L. F. Lira-Barragán, J. M. Ponce-Ortega, M. Serna-González, and M. M. ElHalwagi, "Optimal design of process energy systems integrating sustainable considerations," Energy, vol. 76, pp. 139-160, 2014.

[63] L. M. Julian-Duran, A. P. Ortiz-Espinoza, M. M. El-Halwagi, and A. Jimenez-Gutierrez, "Techno-Economic Assessment and Environmental Impact of Shale Gas Alternatives to Methanol," ACS Sustain. Chem. Eng., vol. 2, pp. 2338-2344, 2014.

[64] D. Yue, F. You, and S. W. Snyder, "Biomass-to-bioenergy and biofuel supply chain optimization: Overview, key issues and challenges," Comput. Chem. Eng., vol. 66, pp. 36-56, 2014.

[65] "Global Guidance Principles for Life Cycle Assessment Databases A Basis for Greener Processes and Products," UNEP/SETAC Life Cycle Initiative, Paris, France DTI/1410/PA, 2011.

[66] A. Marvuglia, E. Benetto, S. Rege, and C. Jury, "Modelling approaches for consequential life-cycle assessment (C-LCA) of bioenergy: Critical review 
and proposed framework for biogas production," Renew. Sustainable Energy Rev., vol. 25, pp. 768-781, 2013.

[67] R. J. Plevin, M. A. Delucchi, and F. Creutzig, "Using Attributional Life Cycle Assessment to Estimate Climate-Change Mitigation Benefits Misleads Policy Makers," J. Ind. Ecol., vol. 18, pp. 73-83, 2014.

[68] J. M. Earles and A. Halog, "Consequential life cycle assessment: a review," Int. J. Life Cycle Assess., vol. 16, pp. 445-453, 2011.

[69] A. Zamagni, J. Guinee, R. Heijungs, P. Masoni, and A. Raggi, "Lights and shadows in consequential LCA," Int. J. Life Cycle Assess., vol. 17, pp. 904918, 2012.

[70] B. P. Weidema, "Market aspects in product life cycle inventory methodology," J. Clean. Prod., vol. 1, pp. 161-166, 1993.

[71] M. A. Curran, M. Mann, and G. Norris, "The international workshop on electricity data for life cycle inventories," J. Clean. Prod., vol. 13, pp. 853862, 2005.

[72] R. Dalgaard, J. Schmidt, N. Halberg, P. Christensen, M. Thrane, and W. A. Pengue, "LCA of soybean meal," Int. J. Life Cycle Assess., vol. 13, pp. 240254, 2008.

[73] J. Reinhard and R. Zah, "Global environmental consequences of increased biodiesel consumption in Switzerland: consequential life cycle assessment," J. Clean. Prod., vol. 17, pp. S46-S56, 2009.

[74] J. H. Schmidt and B. P. Weidema, "Shift in the marginal supply of vegetable oil," Int. J. Life Cycle Assess., vol. 13, pp. 235-239, 2008.

[75] T. Ekvall, "A market-based approach to allocation at open-loop recycling," Resour. Conserv. Recy., vol. 29, pp. 91-109, 2000.

[76] T. Ekvall and A. S. G. Andrae, "Attributional and consequential environmental assessment of the shift to lead-free solders," Int. J. Life Cycle Assess., vol. 11, pp. 344-353, 2006.

[77] D. Rajagopal, "A Step Towards a General Framework for Consequential Life Cycle Assessment," J. Ind. Ecol., DOI: 10.1111/jiec.12433, 2016.

[78] T. Searchinger, R. Heimlich, R. A. Houghton, F. Dong, A. Elobeid, J. Fabiosa, et al., "Use of U.S. Croplands for Biofuels Increases Greenhouse Gases Through Emissions from Land-Use Change," Science, vol. 319, pp. 1238-1240, 2008.

[79] J. M. Earles, A. Halog, P. Ince, and K. Skog, "Integrated Economic Equilibrium and Life Cycle Assessment Modeling for Policy-based Consequential LCA," J. Ind. Ecol., vol. 17, pp. 375-384, 2013.

[80] J. Kløverpris, H. Wenzel, and P. H. Nielsen, "Life cycle inventory modelling of land use induced by crop consumption," Int. J. Life Cycle Assess., vol. 13, p. 13, 2007. 
[81] E. Igos, B. Rugani, S. Rege, E. Benetto, L. Drouet, and D. S. Zachary, "Combination of equilibrium models and hybrid life cycle-input-output analysis to predict the environmental impacts of energy policy scenarios," Appl. Energy, vol. 145, pp. 234-245, 2015.

[82] Y. Yang, "Two sides of the same coin: consequential life cycle assessment based on the attributional framework," J. Clean. Prod., vol. 127, pp. 274-281, 2016.

[83] M. Spielmann, P. de Haan, and R. W. Scholz, "Environmental rebound effects of high-speed transport technologies: a case study of climate change rebound effects of a future underground maglev train system," J. Clean. Prod., vol. 16, pp. 1388-1398, 2008.

[84] J. Thiesen, T. S. Cluistensen, T. G. Kristensen, R. D. Andersen, B. Brunoe, T. K. Gregersen, et al., "Rebound effects of price differences," Int. J. Life Cycle Assess., vol. 13, pp. 104-114, 2008.

[85] B. A. Sandén and M. Karlström, "Positive and negative feedback in consequential life-cycle assessment," J. Clean. Prod., vol. 15, pp. 1469-1481, 2007.

[86] A. Kätelhön, N. von der Assen, S. Suh, J. Jung, and A. Bardow, "IndustryCost-Curve Approach for Modeling the Environmental Impact of Introducing New Technologies in Life Cycle Assessment," Environ. Sci. Technol., vol. 49, pp. 7543-7551, 2015.

[87] A. Kätelhön, A. Bardow, and S. Suh, "Stochastic Technology Choice Model for Consequential Life Cycle Assessment," Environ. Sci. Technol., vol. 50, pp. 12575-12583, 2016.

[88] M. Thrane, "LCA of Danish fish products - New methods and insights," Int. J. Life Cycle Assess., vol. 11, pp. 66-74, 2006.

[89] M. A. Thomassen, R. Dalgaard, R. Heijungs, and I. de Boer, "Attributional and consequential LCA of milk production," Int. J. Life Cycle Assess., vol. 13, pp. 339-349, 2008.

[90] R. Dalgaard, J. Schmidt, and A. Flysjo, "Generic model for calculating carbon footprint of milk using four different life cycle assessment modelling approaches," J. Clean. Prod., vol. 73, pp. 146-153, 2014.

[91] J. H. Schmidt, "Comparative life cycle assessment of rapeseed oil and palm oil," Int. J. Life Cycle Assess., vol. 15, pp. 183-197, 2010.

[92] J. H. Schmidt, "Life cycle assessment of five vegetable oils," J. Clean. Prod., vol. 87, pp. 130-138, 2015.

[93] T. Silalertruksa, S. H. Gheewala, and M. Sagisaka, "Impacts of Thai bioethanol policy target on land use and greenhouse gas emissions," Appl. Energy, vol. 86, pp. S170-S177, 2009. 
[94] B. M. Smyth and J. D. Murphy, "The indirect effects of biofuels and what to do about them: the case of grass biomethane and its impact on livestock," Biofuel. Bioprod. Bior., vol. 5, pp. 165-184, 2011.

[95] D. Tonini, L. Hamelin, H. Wenzel, and T. Astrup, "Bioenergy Production from Perennial Energy Crops: A Consequential LCA of 12 Bioenergy Scenarios including Land Use Changes," Environ. Sci. Technol., vol. 46, pp. 13521-13530, 2012.

[96] N. Escobar, J. Ribal, G. Clemente, and N. Sanjuan, "Consequential LCA of two alternative systems for biodiesel consumption in Spain, considering uncertainty," J. Clean. Prod., vol. 79, pp. 61-73, 2014.

[97] L. Hamelin, I. Naroznova, and H. Wenzel, "Environmental consequences of different carbon alternatives for increased manure-based biogas," Appl. Energy, vol. 114, pp. 774-782, 2014.

[98] M. E. Hums, R. A. Cairncross, and S. Spatari, "Life-Cycle Assessment of Biodiesel Produced from Grease Trap Waste," Environ. Sci. Technol., vol. 50, pp. 2718-2726, 2016.

[99] D. Styles, E. M. Dominguez, and D. Chadwick, "Environmental balance of the UK biogas sector: An evaluation by consequential life cycle assessment," Sci. Total Environ., vol. 560-561, pp. 241-253, 2016.

[100] D. Tonini, L. Hamelin, M. Alvarado-Morales, and T. F. Astrup, "GHG emission factors for bioelectricity, biomethane, and bioethanol quantified for 24 biomass substrates with consequential life-cycle assessment," Bioresour. Technol., vol. 208, pp. 123-133, 2016.

[101] T. Rehl, J. Lansche, and J. Muller, "Life cycle assessment of energy generation from biogas-Attributional vs. consequential approach," Renew. Sustainable Energy Rev., vol. 16, pp. 3766-3775, 2012.

[102] R. A. Kerr, "Natural Gas From Shale Bursts Onto the Scene," Science, vol. 328, pp. 1624-1626, 2010.

[103] J. J. Siirola, "The Impact of Shale Gas in the Chemical Industry," AIChE J., vol. 60, pp. 810-819, 2014.

[104] "Annual energy outlook 2017," The U.S. Energy Information Administration, Washington, D.C., U.S.A. AEO2017.

[105] J. F. Goellner. (2012) Expanding the shale gas infrastructure. Chem Eng Prog. 49-59.

[106] J. G. Speight, "Chapter 4 - Shale Gas Properties and Processing," in Shale Gas Production Processes, J. G. Speight, Ed., ed Boston: Gulf Professional Publishing, 2013, pp. 101-119.

[107] J. Gao and F. You, "Shale Gas Supply Chain Design and Operations toward Better Economic and Life Cycle Environmental Performance: MINLP 
Model and Global Optimization Algorithm," ACS Sustain. Chem. Eng., vol. 3, pp. 1282-1291, 2015.

[108] J. Martinez-Gomez, F. Nápoles-Rivera, J. M. Ponce-Ortega, and M. M. ElHalwagi, "Optimization of the production of syngas from shale gas with economic and safety considerations," Appl Therm Eng, vol. 110, pp. 678685, 2017.

[109] "Modern Shale Gas Development in the United States: An Update " The National Renewable Energy Laboratory, Golden, CO, U.S.A.

[110] M. B. Yang and F. Q. You, "Comparative Techno-Economic and Environmental Analysis of Ethylene and Propylene Manufacturing from Wet Shale Gas and Naphtha," Ind. Eng. Chem. Res., vol. 56, pp. 4038-4051, 2017.

[111] C. He and F. You, "Deciphering the true life cycle environmental impacts and costs of the mega-scale shale gas-to-olefins projects in the United States," Energ. Environ. Sci., vol. 9, pp. 820-840, 2016.

[112] D. C. Cafaro and I. E. Grossmann, "Strategic planning, design, and development of the shale gas supply chain network," AIChE J., vol. 60, pp. 2122-2142, 2014.

[113] J. Gao and F. You, "Optimal Design and Operations of Supply Chain Networks for Water Management in Shale Gas Production: MILFP Model and Algorithms for the Water-Energy Nexus," AIChE J., vol. 61, pp. 11841208, 2015.

[114] L. F. Lira-Barragan, J. M. Ponce-Ortega, G. Guillen-Gosalbez, and M. M. ElHalwagi, "Optimal Water Management under Uncertainty for Shale Gas Production," Ind. Eng. Chem. Res., vol. 55, pp. 1322-1335, 2016.

[115] K. Arredondo-Ramírez, J. M. Ponce-Ortega, and M. M. El-Halwagi, "Optimal planning and infrastructure development for shale gas production," Energ. Convers. Manage., vol. 119, pp. 91-100, 2016.

[116] J. Gong, M. Yang, and F. You, "A systematic simulation-based process intensification method for shale gas processing and NGLs recovery process systems under uncertain feedstock compositions," Comput. Chem. Eng., vol. 105, pp. 259-275, 2017.

[117] J. Gao and F. You, "Deciphering and handling uncertainty in shale gas supply chain design and optimization: Novel modeling framework and computationally efficient solution algorithm," AIChE J., vol. 61, pp. 37393755, 2015.

[118] M. Martín and I. E. Grossmann, "Process Optimization of FT-Diesel Production from Lignocellulosic Switchgrass," Ind. Eng. Chem. Res., vol. 50, pp. 13485-13499, 2011. 
[119] S. Hosseini, K. Barker, and J. E. Ramirez-Marquez, "A review of definitions and measures of system resilience," Reliab. Eng. Syst. Safe., vol. 145, pp. 4761, 2016.

[120] R. Bhamra, S. Dani, and K. Burnard, "Resilience: the concept, a literature review and future directions," Int. J. Prod. Res., vol. 49, pp. 5375-5393, 2011.

[121] M. Ouyang, L. Duenas-Osorio, and X. Min, "A three-stage resilience analysis framework for urban infrastructure systems," Struct. Saf., vol. 36-37, pp. 23-31, 2012.

[122] B. Allenby and J. Fink, "Toward Inherently Secure and Resilient Societies," Science, vol. 309, pp. 1034-1036, 2005.

[123] E. Hollnagel, D. D. Woods, and N. Leveson, Resilience engineering: Concepts and precepts, Farnham, UK: Ashgate Publishing, Ltd., 2007.

[124] P. Martin-Breen and J. M. Anderies, "Resilience: A literature review," IDS, Brighton, UK, 2011.

[125] J. Fiksel, "Designing resilient, sustainable systems," Environ. Sci. Technol., vol. 37, pp. 5330-5339, 2003.

[126] R. Francis and B. Bekera, "A metric and frameworks for resilience analysis of engineered and infrastructure systems," Reliab. Eng. Syst. Safe., vol. 121, pp. 90-103, 2014.

[127] C. S. Holling, "Resilience and Stability of Ecological Systems," Annu. Rev. Ecol. Evol. Syst., vol. 4, pp. 1-23, 1973.

[128] M. Bruneau, S. E. Chang, R. T. Eguchi, G. C. Lee, T. D. O'Rourke, A. M. Reinhorn, et al., "A framework to quantitatively assess and enhance the seismic resilience of communities," Earthq. Spectra, vol. 19, pp. 733-752, 2003.

[129] D. A. Reed, K. C. Kapur, and R. D. Christie, "Methodology for Assessing the Resilience of Networked Infrastructure," IEEE Syst. J., vol. 3, pp. 174-180, 2009.

[130] P. Bocchini and D. M. Frangopol, "Optimal Resilience- and Cost-Based Postdisaster Intervention Prioritization for Bridges along a Highway Segment," J. Bridge Eng., vol. 17, pp. 117-129, 2012.

[131] S. E. Chang and M. Shinozuka, "Measuring improvements in the disaster resilience of communities," Earthq. Spectra, vol. 20, pp. 739-755, 2004.

[132] M. Bruneau and A. Reinhorn, "Exploring the concept of seismic resilience for acute care facilities," Earthq. Spectra, vol. 23, pp. 41-62, 2007.

[133] G. P. Cimellaro, A. M. Reinhorn, and M. Bruneau, "Seismic resilience of a hospital system," Struct. Infrastruct. E., vol. 6, pp. 127-144, 2010.

[134] M. Morari, "Design of resilient processing plants-III: A general framework for the assessment of dynamic resilience," Chem. Eng. Sci., vol. 38, pp. 1881-1891, 1983. 
[135] E. Vugrin, D. Warren, M. Ehlen, and R. C. Camphouse, "A Framework for Assessing the Resilience of Infrastructure and Economic Systems," in Sustainable and Resilient Critical Infrastructure Systems, K. Gopalakrishnan and S. Peeta, Eds., ed: Springer Berlin Heidelberg, 2010, pp. 77-116.

[136] N. Xu, S. D. Guikema, R. A. Davidson, L. K. Nozick, Z. Çağnan, and K. Vaziri, "Optimizing scheduling of post-earthquake electric power restoration tasks," Earthq. Eng. Struct. D, vol. 36, pp. 265-284, 2007.

[137] N. Sahebjamnia, S. A. Torabi, and S. A. Mansouri, "Integrated business continuity and disaster recovery planning: Towards organizational resilience," Eur. J. Oper. Res., vol. 242, pp. 261-273, 2015.

[138] E. D. Vugrin, M. Turnquist, and N. Brown, "Optimal recovery sequencing for critical infrastructure resilience assessment," The Sandia National Laboratories, Albuquerque, NM, USA SAND2010-6237, 2010.

[139] J. R. Birge and F. Louveaux, Introduction to stochastic programming. Springer Science \& Business Media, 2011.

[140] D. Bertsimas and M. Sim, "The price of robustness," Oper Res, vol. 52, pp. 35-53, 2004.

[141] X. X. Lin, S. L. Janak, and C. A. Floudas, "A new robust optimization approach for scheduling under uncertainty: I. Bounded uncertainty," Comput. Chem. Eng., vol. 28, pp. 1069-1085, 2004.

[142] Y. Yuan, Z. Li, and B. Huang, "Robust optimization under correlated uncertainty: Formulations and computational study," Comput. Chem. Eng., vol. 85, pp. 58-71, 2016.

[143] P. Garcia-Herreros, J. M. Wassick, and I. E. Grossmann, "Design of Resilient Supply Chains with Risk of Facility Disruptions," Ind. Eng. Chem. Res., vol. 53, pp. 17240-17251, 2014.

[144] S. R. Cardoso, A. Paula Barbosa-Póvoa, S. Relvas, and A. Q. Novais, "Resilience metrics in the assessment of complex supply-chains performance operating under demand uncertainty," Omega, vol. 56, pp. 5373, 2015.

[145] W. Yuan, J. Wang, F. Qiu, C. Chen, C. Kang, and B. Zeng, "Robust Optimization-Based Resilient Distribution Network Planning Against Natural Disasters," IEEE Trans. Smart Grid, vol. 7, pp. 2817-2826, 2016.

[146] M. Turnquist and E. Vugrin, "Design for resilience in infrastructure distribution networks," Environ. Syst. Deci., vol. 33, pp. 104-120, 2013.

[147] J. G. Jin, L. C. Tang, L. Sun, and D.-H. Lee, "Enhancing metro network resilience via localized integration with bus services," Transport. Res. E Logistics Transport. Rev., vol. 63, pp. 17-30, 2014. 
[148] I. E. Grossmann, B. A. Calfa, and P. Garcia-Herreros, "Evolution of concepts and models for quantifying resiliency and flexibility of chemical processes," Comput. Chem. Eng., vol. 70, pp. 22-34, 2014.

[149] D. A. Straub and I. E. Grossmann, "Integrated stochastic metric of flexibility for systems with discrete state and continuous parameter uncertainties," Comput. Chem. Eng., vol. 14, pp. 967-985, 1990.

[150] S. Rajagopalan, N. V. Sahinidis, S. Amaran, A. Agarwal, S. J. Bury, B. Sharda, et al., "Risk analysis of turnaround reschedule planning in integrated chemical sites," Comput. Chem. Eng., DOI: 10.1016/j.compchemeng.2017.04.017.

[151] T. V. Thomaidis and E. N. Pistikopoulos, "Optimal design of flexible and reliable process systems," IEEE Transactions on Reliability, vol. 44, pp. 243250, 1995.

[152] Y. Ye, I. E. Grossmann, and J. M. Pinto, "MINLP models for optimal design of reliable chemical plants," in FOCAPO/CPC 2017, Tucson, Arizona, 2017.

[153] S. Terrazas-Moreno, I. E. Grossmann, J. M. Wassick, and S. J. Bury, "Optimal design of reliable integrated chemical production sites," Comput. Chem. Eng., vol. 34, pp. 1919-1936, 2010.

[154] L. T. T. Dinh, H. Pasman, X. D. Gao, and M. S. Mannan, "Resilience engineering of industrial processes: Principles and contributing factors," $J$. Loss Prevent. Proc., vol. 25, pp. 233-241, 2012.

[155] D. Bertsimas, E. Litvinov, X. A. Sun, J. Zhao, and T. Zheng, "Adaptive Robust Optimization for the Security Constrained Unit Commitment Problem," IEEE Trans. Power Syst., vol. 28, pp. 52-63, 2013.

[156] N. H. Lappas and C. E. Gounaris, "Multi-stage adjustable robust optimization for process scheduling under uncertainty," AIChE J., vol. 62, pp. 1646-1667, 2016.

[157] I. E. Grossmann, "Mixed-integer nonlinear programming techniques for the synthesis of engineering systems," Research in Engineering Design, vol. 1, pp. 205-228, 1990.

[158] N. Hanagata, T. Takeuchi, Y. Fukuju, D. J. Barnes, and I. Karube, "Tolerance of Microalgae to High Co-2 and High-Temperature," Phytochemistry, vol. 31, pp. 3345-3348, 1992.

[159] E. Frank, J. Han, I. Palou-Rivera, A. Elgowainy, and M. Wang, "Life-Cycle Analysis of Algal Lipid Fuels with the GREET Model," U.S. Department of Energy, Office of Scientific and Technical Information, Oak Ridge, TN, USA ANL/ESD/11-5, 2011.

[160] C. S. Q. Association, "California Stormwater BMP Handbook-New Development and Redevelopment," California Stormwater Quality Association, Menlo Park, CA, 2003. 
[161] M. R. Granados, F. G. Acien, C. Gomez, J. M. Fernandez-Sevilla, and E. M. Grima, "Evaluation of flocculants for the recovery of freshwater microalgae," Bioresour. Technol., vol. 118, pp. 102-110, 2012.

[162] L. K. Wang, N. K. Shammas, and Y. T. Hung, Biosolids Treatment Processes: Volume 6: Humana Press, 2007.

[163] N. Uduman, Y. Qi, M. K. Danquah, G. M. Forde, and A. Hoadley, "Dewatering of microalgal cultures: A major bottleneck to algae-based fuels," J Renew Sustain Ener, vol. 2, 2010.

[164] J. Y. Lee, C. Yoo, S. Y. Jun, C. Y. Ahn, and H. M. Oh, "Comparison of several methods for effective lipid extraction from microalgae," Bioresour. Technol., vol. 101, pp. S75-S77, 2010.

[165] R. Halim, M. K. Danquah, and P. A. Webley, "Extraction of oil from microalgae for biodiesel production: A review," Biotechnol. Adv., vol. 30, pp. 709-732, 2012.

[166] A. Plus, "Aspen Plus user guide," Aspen Technology Limited, Cambridge, Massachusetts, United States, 2003.

[167] R. L. Smith, H. Inomata, M. Kanno, and K. Arai, "Energy analysis of supercritical carbon dioxide extraction processes," J Supercrit Fluid, vol. 15, pp. 145-156, 1999.

[168] M. Bravi, R. Bubbico, F. Manna, and N. Verdone, "Process optimisation in sunflower oil extraction by supercritical CO2," Chem. Eng. Sci., vol. 57, pp. 2753-2764, 2002.

[169] F. R. Ma and M. A. Hanna, "Biodiesel production: a review," Bioresour. Technol., vol. 70, pp. 1-15, 1999.

[170] L. C. Meher, D. V. Sagar, and S. N. Naik, "Technical aspects of biodiesel production by transesterification - a review," Renew. Sustainable Energy Rev., vol. 10, pp. 248-268, 2006.

[171] A. A. Apostolakou, I. K. Kookos, C. Marazioti, and K. C. Angelopoulos, "Techno-economic analysis of a biodiesel production process from vegetable oils," Fuel Process Technol, vol. 90, pp. 1023-1031, 2009.

[172] A. H. West, D. Posarac, and N. Ellis, "Assessment of four biodiesel production processes using HYSYS. Plant," Bioresour. Technol., vol. 99, pp. 6587-6601, 2008.

[173] L. F. Sotoft, B. G. Rong, K. V. Christensen, and B. Norddahl, "Process simulation and economical evaluation of enzymatic biodiesel production plant," Bioresour. Technol., vol. 101, pp. 5266-5274, 2010.

[174] A. Ebshish, Z. Yaakob, Y. H. Taufiq-Yap, A. Bshish, and S. M. Tasirin, "Review of hydrogen production via glycerol reforming," P I Mech Eng a-J Pow, vol. 226, pp. 1060-1075, 2012. 
[175] S. B. Jones, C. Valkenburg, C. W. Walton, D. C. Elliott, J. E. Holladay, D. J. Stevens, et al., Production of gasoline and diesel from biomass via fast pyrolysis, hydrotreating and hydrocracking: a design case: Pacific Northwest National Laboratory Richland, WA, 2009.

[176] G. W. Huber, S. Iborra, and A. Corma, "Synthesis of transportation fuels from biomass: Chemistry, catalysts, and engineering," Chem Rev, vol. 106, pp. 4044-4098, 2006.

[177] N. Hajjaji, A. Chahbani, Z. Khila, and M. N. Pons, "A comprehensive energy-exergy-based assessment and parametric study of a hydrogen production process using steam glycerol reforming," Energy, vol. 64, pp. 473-483, 2014.

[178] T. Valliyappan, D. Ferdous, N. N. Bakhshi, and A. K. Dalai, "Production of hydrogen and syngas via steam gasification of glycerol in a fixed-bed reactor," Top Catal, vol. 49, pp. 59-67, 2008.

[179] Y. Choi and H. G. Stenger, "Water gas shift reaction kinetics and reactor modeling for fuel cell grade hydrogen," J Power Sources, vol. 124, pp. 432439, 2003.

[180] K. H. Lin, A. C. C. Chang, W. H. Lin, S. H. Chen, C. Y. Chang, and H. F. Chang, "Autothermal steam reforming of glycerol for hydrogen production over packed-bed and $\mathrm{Pd} / \mathrm{Ag}$ alloy membrane reactors," Int $J$ Hydrogen Energ, vol. 38, pp. 12946-12952, 2013.

[181] H. Kooijman. (2006, 8/18/2014). Air Separation Unit with Pure Argon Recovery (Simplified). Available: http://chemsep.org/downloads/data/CScasebook ASU.pdf

[182] Y. Guo, X. H. Liu, M. U. Azmat, W. J. Xu, J. W. Ren, Y. Q. Wang, et al., "Hydrogen production by aqueous-phase reforming of glycerol over Ni-B catalysts," Int J Hydrogen Energ, vol. 37, pp. 227-234, 2012.

[183] M. Pagliaro and M. Rossi, The future of glycerol, Cambridge: Royal Society of Chemistry, 2010.

[184] P. Bloom, "Generating high purity, biobased propylene glycol; by product inhibition; reactor system," ed: Google Patents, 2011.

[185] M. W. M. Tuck, "Process for the hydrogenation of glycerol to propylene glycol," ed: Google Patents, 2012.

[186] E. Vlad, C. S. Bildea, and G. Bozga, "Robust Optimal Design of a Glycerol Etherification Process," Chem Eng Technol, vol. 36, pp. 251-258, 2013.

[187] J. I. Choi and S. Y. Lee, "Process analysis and economic evaluation for poly(3-hydroxybutyrate) production by fermentation," Bioprocess Eng, vol. 17, pp. 335-342, 1997. 
[188] J. A. Posada, J. M. Naranjo, J. A. Lopez, J. C. Higuita, and C. A. Cardona, "Design and analysis of poly-3-hydroxybutyrate production processes from crude glycerol," Process Biochem, vol. 46, pp. 310-317, 2011.

[189] K. H. Rostkowski, C. S. Criddle, and M. D. Lepech, "Cradle-to-Gate Life Cycle Assessment for a Cradle-to-Cradle Cycle: Biogas-to-Bioplastic (and Back)," Environ. Sci. Technol., vol. 46, pp. 9822-9829, 2012.

[190] G.-K. Plattner, T. Stocker, P. Midgley, and M. Tignor, "IPCC expert meeting on the science of alternative metrics," 2009.

[191] K. Sander and G. S. Murthy, "Life cycle analysis of algae biodiesel," Int. J. Life Cycle Assess., vol. 15, pp. 704-714, 2010.

[192] H. Passell, H. Dhaliwal, M. Reno, B. Wu, A. Ben Amotz, E. Ivry, et al., "Algae biodiesel life cycle assessment using current commercial data," $J$. Environ. Manage., vol. 129, pp. 103-111, 2013.

[193] P. K. Campbell, T. Beer, and D. Batten, "Life cycle assessment of biodiesel production from microalgae in ponds," Bioresour. Technol., vol. 102, pp. 50-56, 2011.

[194] DOT. (2013, September 2014). Table 6 Shipment Characteristics by TwoDigit Commodity for the Unied States: 2012. Available: http://www.rita.dot.gov/bts/sites/rita.dot.gov.bts/files/publications/commod ity flow_survey/2012/united_states/table6.html

[195] (2016, November 2016). Ecoinvent database v3.3 (2016 ed.). Available: http://www.ecoinvent.ch/

[196] B. S. Institution, "ISO 14040:2006 Environmental Management-Life Cycle Assessment-Principles and Framework," ed. London, 2006.

[197] D. Yue, M. A. Kim, and F. You, "Design of Sustainable Product Systems and Supply Chains with Life Cycle Optimization Based on Functional Unit: General Modeling Framework, Mixed-Integer Nonlinear Programming Algorithms and Case Study on Hydrocarbon Biofuels," ACS Sustain. Chem. Eng., vol. 1, pp. 1003-1014, 2013.

[198] L. Lardon, A. Helias, B. Sialve, J. P. Steyer, and O. Bernard, "Life-Cycle Assessment of Biodiesel Production from Microalgae," Environ. Sci. Technol., vol. 43, pp. 6475-6481, 2009.

[199] C. L. Hwang and A. S. M. Masud, Multiple objective decision makingmethods and applications, Heidelberg, Germany: Springer-Verlag Berlin Heidelberg, 1979.

[200] F. You and I. E. Grossmann, "Stochastic Inventory Management for Tactical Process Planning Under Uncertainties: MINLP Models and Algorithms," AIChE J., vol. 57, pp. 1250-1277, 2011. 
[201] F. You, J. M. Pinto, I. E. Grossmann, and L. Megan, "Optimal DistributionInventory Planning of Industrial Gases. II. MINLP Models and Algorithms for Stochastic Cases," Ind. Eng. Chem. Res., vol. 50, pp. 2928-2945, 2011.

[202] Z. Zhong and F. You, "Globally convergent exact and inexact parametric algorithms for solving large-scale mixed-integer fractional programs and applications in process systems engineering," Comput. Chem. Eng., vol. 61, pp. 90-101, 2014.

[203] R. E. Rosenthal, "GAMS - a user's guide," GAMS Development Corporation, Washington, DC, USA, 2016.

[204] M. Tawarmalani and N. V. Sahinidis, "A polyhedral branch-and-cut approach to global optimization," Math Program, vol. 103, pp. 225-249, 2005.

[205] J. Dufour, D. P. Serrano, J. L. Galvez, J. Moreno, and C. Garcia, "Life cycle assessment of processes for hydrogen production. Environmental feasibility and reduction of greenhouse gases emissions," Int J Hydrogen Energ, vol. 34, pp. 1370-1376, 2009.

[206] C. Koroneos, A. Dompros, G. Roumbas, and N. Moussiopoulos, "Life cycle assessment of hydrogen fuel production processes," Int J Hydrogen Energ, vol. 29, pp. 1443-1450, 2004.

[207] ADM. (2014, September 2014). Life Cycle Analysis. Available: http://www.adm.com/en-US/products/evolution/PropyleneGlycol/Pages/Life Cycle Analysis.aspx

[208] Zemea. (September 2014). Purity and Renewable Performance. Available: http://www.duponttateandlyle.com/sites/default/files/ZemeaHI\%26I lifeCy cleAnalysis.pdf

[209] C. Beatrice, G. Di Blasio, M. Lazzaro, C. Cannilla, G. Bonura, F. Frusteri, et al., "Technologies for energetic exploitation of biodiesel chain derived glycerol: Oxy-fuels production by catalytic conversion," Appl. Energy, vol. 102, pp. 63-71, 2013.

[210] S. Kim and B. E. Dale, "Life cycle assessment study of biopolymers (Polyhydroxyalkanoates) derived from no-tilled corn," Int. J. Life Cycle Assess., vol. 10, pp. 200-210, 2005.

[211] A. Roes, M. Pietrini, E. Chiellini, and M. Patel, "Environmental life cycle studies of poly (hydroxybutyrate)-and polypropylene-based composites," Journal of nanostructured polymers and nanocomposites, vol. 3, pp. 22-32, 2007.

[212] DOE. (2015, January 2016). Fuel Prices. Available: http://www.afdc.energy.gov/fuels/prices.html

[213] D. Yue, G. Guillen-Gosalbez, and F. You, "Global Optimization of LargeScale Mixed-Integer Linear Fractional Programming Problems: A 
Reformulation-Linearization Method and Process Scheduling Applications," AIChE J., vol. 59, pp. 4255-4272, 2013.

[214] B. H. Gebreslassie, M. Slivinsky, B. L. Wang, and F. You, "Life cycle optimization for sustainable design and operations of hydrocarbon biorefinery via fast pyrolysis, hydrotreating and hydrocracking," Comput Chem Eng, vol. 50, pp. 71-91, 2013.

[215] O. M. Hernández-Calderón, J. M. Ponce-Ortega, J. R. Ortiz-del-Castillo, M. E. Cervantes-Gaxiola, J. Milán-Carrillo, M. Serna-González, et al., "Optimal Design of Distributed Algae-Based Biorefineries Using CO2Emissions from Multiple Industrial Plants," Ind. Eng. Chem. Res., DOI: 10.1021/acs.iecr.5b01684, 2016.

[216] J. Kim, M. J. Realff, and J. H. Lee, "Optimal design and global sensitivity analysis of biomass supply chain networks for biofuels under uncertainty," Comput. Chem. Eng., vol. 35, pp. 1738-1751, 2011.

[217] F. Glover, "Improved Linear Integer Programming Formulations of Nonlinear Integer Problems," Manage Sci, vol. 22, pp. 455-460, 1975.

[218] S. Zionts, "Programming with Linear Fractional Functionals," Nav Res Logist $Q$ vol. 15, pp. 449-\&, 1968.

[219] Y. F. Chu and F. Q. You, "Integration of Scheduling and Dynamic Optimization of Batch Processes under Uncertainty: Two-Stage Stochastic Programming Approach and Enhanced Generalized Benders Decomposition Algorithm," Ind. Eng. Chem. Res., vol. 52, pp. 16851-16869, 2013.

[220] A. Ben-Tal, A. Goryashko, E. Guslitzer, and A. Nemirovski, "Adjustable robust solutions of uncertain linear programs," Math Program, vol. 99, pp. 351-376, 2004.

[221] P. J. Goulart, E. C. Kerrigan, and J. A. Maciejowski, "Optimization over state feedback policies for robust control with constraints," Automatica, vol. 42, pp. 523-533, 2006.

[222] A. Ben-Tal, B. Golany, A. Nemirovski, and J.-P. Vial, "Retailer-Supplier Flexible Commitments Contracts: A Robust Optimization Approach," Manufacturing \& Service Operations Management, vol. 7, pp. 248-271, 2005.

[223] D. Bertsimas, D. A. Iancu, and P. A. Parrilo, "A Hierarchy of Near-Optimal Policies for Multistage Adaptive Optimization," IEEE T Automat Contr, vol. 56, pp. 2803-2818, 2011.

[224] X. Chen, M. Sim, P. Sun, and J. W. Zhang, "A linear decision-based approximation approach to stochastic programming," Oper Res, vol. 56, pp. 344-357, 2008.

[225] D. Bertsimas and C. Caramanis, "Finite Adaptability in Multistage Linear Optimization," IEEE T Automat Contr, vol. 55, pp. 2751-2766, 2010. 
[226] A. Thiele, T. Terry, and M. Epelman, "Robust Linear Optimization With Recourse," University of Michigan TR09-01, 2010.

[227] R. W. Jiang, J. H. Wang, and Y. P. Guan, "Robust Unit Commitment With Wind Power and Pumped Storage Hydro," IEEE Trans. Power Syst., vol. 27, pp. 800-810, 2012.

[228] B. Zeng and L. Zhao, "Solving two-stage robust optimization problems using a column-and-constraint generation method," Oper. Res. Lett., vol. 41, pp. 457-461, 2013.

[229] D. Yue and F. You, "Optimal supply chain design and operations under multi-scale uncertainties: Nested stochastic robust optimization modeling framework and solution algorithm," AIChE J., vol. 62, pp. 3041-3055, 2016.

[230] J. Gong, D. J. Garcia, and F. You, "Unraveling Optimal Biomass Processing Routes from Bioconversion Product and Process Networks under Uncertainty: An Adaptive Robust Optimization Approach," ACS Sustain. Chem. Eng., vol. 4, pp. 3160-3173, 2016.

[231] EIA. (2015, January 2016). ELECTRICITY DATA BROWSER. Available: http://www.eia.gov/electricity/data/browser/\#/topic/7?agg=0,1\&geo=g\&ends $\mathrm{ec}=\mathrm{vg} \&$ linechart=ELEC.PRICE.US-ALL.M $\sim$ ELEC.PRICE.US-

RES.M $\sim$ ELEC.PRICE.US-COM.M $\sim$ ELEC.PRICE.US-

IND.M\&columnchart=ELEC.PRICE.US-ALL.M ${ }^{\sim}$ ELEC.PRICE.USRES.M $\sim$ ELEC.PRICE.US-COM.M $\sim$ ELEC.PRICE.US-

IND.M\&map=ELEC.PRICE.US-

ALL.M\&freq=M\&start=201411\&end=201511\&ctype=linechart\&ltype=pin\&r type $=$ \&\&maptype $=0 \&$ rse $=0 \&$ pin $=$

[232] ycharts.com. (2016, January 2016). US Producer Price Index: Chemicals and Allied Products: Oxygen. Available: https://ycharts.com/indicators/us_producer_price_index_chemicals_and_all ied products oxygen

[233] indexmundi.com. (2016, January 2016). Urea Monthly Price. Available: http://www.indexmundi.com/commodities/?commodity=urea\&months $=12$

[234] indexmundi.com. (2016, January 2016). DAP fertilizer Monthly Price. Available: $\quad \underline{\text { http://www.indexmundi.com/commodities/?commodity=dap- }}$ fertilizer\&months $=12$

[235] platts.com. (2015, January 2016). HEXANE: EUROPEAN TRUCK PRICES UP; US SPOT PRICES REMAIN STABLE. Available: http://www.platts.com/news-feature/2015/petrochemicals/global-solventsoverview/solvents-hexane-prices

[236] M. Rizwan, M. Zaman, J. H. Lee, and R. Gani, "Optimal processing pathway selection for microalgae-based biorefinery under uncertainty," Comput. Chem. Eng., vol. 82, pp. 362-373, 2015. 
[237] N. G. Mankiw, Principles of Microeconomics, 7th ed. Boston, MA, U.S.A.: Cengage Learning.

[238] B. P. Weidema, C. Bauer, R. Hischier, C. Mutel, T. Nemecek, J. Reinhard, et al., "Overview and methodology: Data quality guideline for the ecoinvent database version 3," Swiss Centre for Life Cycle Inventories, St. Gallen, Switzerland.

[239] D. J. Yue, S. Pandya, and F. Q. You, "Integrating Hybrid Life Cycle Assessment with Multiobjective Optimization: A Modeling Framework," Environ. Sci. Technol., vol. 50, pp. 1501-1509, 2016.

[240] S. Suh and Y. Yang, "On the uncanny capabilities of consequential LCA," Int. J. Life Cycle Assess., vol. 19, pp. 1179-1184, 2014.

[241] I. Vazquez-Rowe, S. Rege, A. Marvuglia, J. Thenie, A. Haurie, and E. Benetto, "Application of three independent consequential LCA approaches to the agricultural sector in Luxembourg," Int. J. Life Cycle Assess., vol. 18, pp. 1593-1604, 2013.

[242] R. Davis, J. Markham, C. Kinchin, N. Grundl, E. C. D. Tan, and D. Humbird, "Process Design and Economics for the Production of Algal Biomass: Algal Biomass Production in Open Pond Systems and Processing Through Dewatering for Downstream Conversion," The National Renewable Energy Laboratory, Golden, CO, U.S.A. NREL/TP-5100-64772.

[243] R. E. Davis, D. B. Fishman, E. D. Frank, M. C. Johnson, S. B. Jones, C. M. Kinchin, et al., "Integrated Evaluation of Cost, Emissions, and Resource Potential for Algal Biofuels at the National Scale," Environ. Sci. Technol., vol. 48, pp. 6035-6042, 2014.

[244] (May 2017). U.S. Energy Mapping System. Available: https://www.eia.gov/state/maps.php

[245] (February 2017). Yara North America opens new Louisiana urea terminal. Available: https://www.icis.com/resources/news/2011/09/28/9496004/yaranorth-america-opens-new-louisiana-urea-terminal/

[246] CFIndustries. (November 2016). Investor Presentation Spring 2016. Available:

http://www.snl.com/Cache/1001207780.PDF?O=PDF\&T=\&Y=\&D=\&FID=10 $\underline{01207780 \& \text { iid }=4533245}$

[247] S. Sun, M. S. Delgado, and J. P. Sesmero, "Dynamic adjustment in agricultural practices to economic incentives aiming to decrease fertilizer application," J. Environ. Manage., vol. 177, pp. 192-201, 2016.

[248] B. Knorr. (2016, November 2016). Weekly Fertilizer Review. Available: http://farmfutures.com/story-weekly-fertilizer-review-0-30765

[249] J. H. Meessen, "Urea," in Ullmann's Encyclopedia of Industrial Chemistry, ed Weinheim, Germany: Wiley-VCH Verlag GmbH \& Co. KGaA, 2000. 
[250] S. E. Amenumey and P. D. Capel, "Fertilizer Consumption and Energy Input for 16 Crops in the United States," Natural Resources Research, vol. 23, pp. 299-309, 2014.

[251] J. E. Sawyer, "Nitrogen Use in Iowa Corn Production," Iowa State University Extension and Outreach, Ames, IA, U.S.A. CROP 3073.

[252] "Crop Production 2015 Summary," The U.S. Department of Agriculture, National Agricultural Statistics Service, Washington, D.C., U.S.A., 2016.

[253] Mosaic. (2014, November 2016). The State of Agriculture and Plant Nutrient Industries. Available: http://www.mosaicco.com/Rahm_Piper_Jaffray_Presentation_8.5.14.pdf

[254] PotashCorp. (2014, November 2016). World Phosphate Acid Uses. Available:

http://www.potashcorp.com/overview/nutrients/phosphate/overview/world -phosphate-acid-uses

[255] D. E. Kaiser, J. A. Lamb, and R. Eliason. (2011, November 2016). Fertilizer Recommendations for Agronomic Crops in Minnesota-Corn. Available: http://www.extension.umn.edu/agriculture/nutrient-management/nutrientlime-guidelines/fertilizer-recommendations-for-agronomic-crops-inminnesota/corn/

[256] D. E. Kaiser, J. A. Lamb, and R. Eliason. (2011, November 2016). Fertilizer Recommendations for Agronomic Crops in Minnesota-Soybean. Available: http://www.extension.umn.edu/agriculture/nutrient-management/nutrientlime-guidelines/fertilizer-recommendations-for-agronomic-crops-inminnesota/soybean/

[257] D. E. Kaiser, J. A. Lamb, and R. Eliason. (2011, November 2016). Fertilizer Recommendations for Agronomic Crops in Minnesota-Wheat. Available: http://www.extension.umn.edu/agriculture/nutrient-management/nutrientlime-guidelines/fertilizer-recommendations-for-agronomic-crops-inminnesota/wheat/

[258] C. Hausman and R. Kellogg, "Welfare and Distributional Implications of Shale Gas," Brookings Pap. Econ. Act., vol. 2015, pp. 71-139, 2016.

[259] EPA. (2011, November 2016). ChemView database. Available: https://java.epa.gov/chemview

[260] "Toxicological Profile for $n$-Hexane," The U.S. Department of Health and Human Services, Public Health Service Agency for Toxic Substances and Disease Registry, Atlanta, GA, U.S.A.

[261] Tenaska. (2011, November 2016). 4.0 ERCOT Market Analysis. Available: https:/hub.globalccsinstitute.com/publications/bridging-commercial-gapcarbon-capture-and-storage/40-ercot-market-analysis 
[262] J. Zarnikau and I. Hallett, "Aggregate industrial energy consumer response to wholesale prices in the restructured Texas electricity market," Energ. Econ., vol. 30, pp. 1798-1808, 2008.

[263] R. McCormick and T. Alleman. (2016, November 2016). Renewable Diesel Fuel. Available: https://cleancities.energy.gov/files/u/news events/document/document url/ 182/McCormick Alleman RD Overview 201607 18.pdf

[264] C. A. Dahl, "Measuring global gasoline and diesel price and income elasticities," Energy Policy, vol. 41, pp. 2-13, 2012.

[265] G. Myhre, D. Shindell, F.-M. Bréon, W. Collins, J. Fuglestvedt, J. Huang, et al., "Anthropogenic and natural radiative forcing," Cambridge University Press, Cambridge, U.K. and New York, NY, U.S.A.

[266] R. Slade and A. Bauen, "Micro-algae cultivation for biofuels: Cost, energy balance, environmental impacts and future prospects," Biomass Bioenergy, vol. 53, pp. 29-38, 2013.

[267] J. Gong and F. You, "Optimal processing network design under uncertainty for producing fuels and value-added bioproducts from microalgae: Twostage adaptive robust mixed integer fractional programming model and computationally efficient solution algorithm," AIChE J., vol. 63, pp. 582-600, 2017.

[268] D. Yue and F. You, "Game-theoretic modeling and optimization of multiechelon supply chain design and operation under Stackelberg game and market equilibrium," Comput. Chem. Eng., vol. 71, pp. 347-361, 2014.

[269] A. K. Pegallapati and E. D. Frank, "Energy use and greenhouse gas emissions from an algae fractionation process for producing renewable diesel," Algal Research, vol. 18, pp. 235-240, 2016.

[270] E. D. Frank, A. Elgowainy, J. Han, and Z. C. Wang, "Life cycle comparison of hydrothermal liquefaction and lipid extraction pathways to renewable diesel from algae," Mitig Adapt Strat Gl, vol. 18, pp. 137-158, 2013.

[271] C. Y. C. Lin and L. Prince, "Gasoline price volatility and the elasticity of demand for gasoline," Energ. Econ., vol. 38, pp. 111-117, 2013.

[272] K. M. Sundaram and G. F. Froment, "Modeling of thermal cracking kinetics-I," Chem. Eng. Sci., vol. 32, pp. 601-608, 1977.

[273] K. M. Sundaram and G. F. Froment, "Modeling of thermal cracking kinetics-II," Chem. Eng. Sci., vol. 32, pp. 609-617, 1977.

[274] L. T. Biegler and I. E. Grossmann, "Retrospective on optimization," Comput. Chem. Eng., vol. 28, pp. 1169-1192, 2004.

[275] Y. K. Salkuyeh and T. A. Adams, "A novel polygeneration process to coproduce ethylene and electricity from shale gas with zero $\mathrm{CO} 2$ emissions 
via methane oxidative coupling," Energ. Convers. Manage., vol. 92, pp. 406420, 2015.

[276] Y. K. Salkuyeh and T. A. Adams, "Co-Production of Olefins, Fuels, and Electricity from Conventional Pipeline Gas and Shale Gas with Near-Zero CO2 Emissions. Part I: Process Development and Technical Performance," Energies, vol. 8, p. 3739, 2015.

[277] Y. K. Salkuyeh and T. A. Adams, "Co-Production of Olefins, Fuels, and Electricity from Conventional Pipeline Gas and Shale Gas with Near-Zero CO2 Emissions. Part II: Economic Performance," Energies, vol. 8, p. 3762, 2015.

[278] J. Kidnay, W. R. Parrish, and D. G. McCartney, "Gas Treating," in Fundamentals of Natural Gas Processing, Second Edition, ed: CRC Press, 2011, pp. 211-241.

[279] H. Benson and R. Parrish, "HiPure process removes CO2/H2S," Hydrocarbon Processing, vol. 53, pp. 81-82, 1974.

[280] GPSA, Gas Processors Suppliers Association Engineering Data Book, 13 ed. Tulsa, Oklahoma, USA, 2012.

[281] J. Kidnay, W. R. Parrish, and D. G. McCartney, "Gas Dehydration," in Fundamentals of Natural Gas Processing, Second Edition, ed: CRC Press, 2011, pp. 243-271.

[282] R. J. Lee, Y. Zhang, J. Yao, J. J. Chen, and D. G. Elliot, "Internal refrigeration for enhanced NGL recovery," 7257966 B2, 2007.

[283] H. Zimmermann and R. Walzl, "Ethylene," in Ullmann's Encyclopedia of Industrial Chemistry, ed: Wiley-VCH Verlag GmbH \& Co. KGaA, 2000.

[284] V. P. Haribal, L. M. Neal, and F. Li, "Oxidative dehydrogenation of ethane under a cyclic redox scheme - Process simulations and analysis," Energy, vol. 119, pp. 1024-1035, 2017.

[285] J. Schulze and M. Homann, "Processes of Separation and Transformation in C4-Chemistry," in C4-Hydrocarbons and Derivatives: Resources, Production, Marketing, ed Berlin, Heidelberg: Springer Berlin Heidelberg, 1989, pp. 35-106.

[286] A. M. Robert, "Dehydrogenation," in Handbook of Petroleum Refining Processes, Third Edition, ed: McGraw Hill Professional, Access Engineering, 2004.

[287] O. S. Pavlov, S. A. Karsakov, and S. Y. Pavlov, "Development of processes for C4 hydrocarbons separation and 1,3-butadiene purification," Theor Found Chem Eng, vol. 45, pp. 858-867, 2011.

[288] X. Yang, X. Yin, and P. Ouyang, "Simulation of 1,3-Butadiene Production Process by Dimethylfomamide Extractive Distillation," Chinese J Chem Eng, vol. 17, pp. 27-35, 2009. 
[289] M. Winterberg, D. Roettger, A. Rix, R. Bukohl, W. Luh, H. Wiederhold, et al., "Preparation of isobutene by dissociation of MTBE," 20120142985 A1, 2015.

[290] S. Ogura and M. Soumai, "Process for separating highly pure butene-1 and butene-2," 1985.

[291] D. T. Allen, V. M. Torres, J. Thomas, D. W. Sullivan, M. Harrison, A. Hendler, et al., "Measurements of methane emissions at natural gas production sites in the United States," P. Natl. Acad. Sci. U.S.A., vol. 110, pp. 17768-73, 2013.

[292] A. T. Dale, V. Khanna, R. D. Vidic, and M. M. Bilec, "Process Based LifeCycle Assessment of Natural Gas from the Marcellus Shale," Environ. Sci. Technol., vol. 47, pp. 5459-5466, 2013.

[293] I. J. Laurenzi and G. R. Jersey, "Life Cycle Greenhouse Gas Emissions and Freshwater Consumption of Marcellus Shale Gas," Environ. Sci. Technol., vol. 47, pp. 4896-4903, 2013.

[294] M. Jiang, C. T. Hendrickson, and J. M. VanBriesen, "Life Cycle Water Consumption and Wastewater Generation Impacts of a Marcellus Shale Gas Well," Environ. Sci. Technol., vol. 48, pp. 1911-1920, 2014.

[295] S. Pfister, S. Vionnet, T. Levova, and S. Humbert, "Ecoinvent 3: assessing water use in LCA and facilitating water footprinting," Int. J. Life Cycle Assess., vol. 21, pp. 1349-1360, 2016.

[296] J. Gao and F. You, "Economic and Environmental Life Cycle Optimization of Noncooperative Supply Chains and Product Systems: Modeling Framework, Mixed-Integer Bilevel Fractional Programming Algorithm, and Shale Gas Application," ACS Sustain. Chem. Eng., DOI: 10.1021/acssuschemeng.7b00002, 2017.

[297] J. Kidnay, W. R. Parrish, and D. G. McCartney, "Overview of Gas Plant Processing," in Fundamentals of Natural Gas Processing, Second ed: CRC Press, 2011, pp. 149-160.

[298] S. A. Holditch, "Getting the gas out of the ground," Chem Eng Prog, vol. 108, pp. 41-48, 2012.

[299] AspenTech, Aspen HYSYS Customization Guide, Burlington, MA, USA: Aspen Technology, Inc., 2010.

[300] M. J. Guerra. (2006, June 2017). Aspen HYSYS Property Packages Overview and Best Practices for Optimum Simulations. Available: http://sites.poli.usp.br/d/pqi2408/BestPracticesOptimumSimulationsHYSYS PropertyPackages.pdf

[301] C. Ruiz, J. Coca, A. Vega, and F. V. Díez, "Extractive Distillation of Hydrocarbons with Dimethylformamide: Experimental and Simulation Data," Ind. Eng. Chem. Res., vol. 36, pp. 4934-4939, 1997. 
[302] AspenTech, Aspen Process Economic Analyzer V7.2 User Guide, Burlington, MA, USA: Aspen Technology, Inc., 2010.

[303] "MATLAB User's Guide," The Mathworks Inc., Natick, MA, 1998.

[304] L. F. Shampine and M. W. Reichelt, "The MATLAB ODE Suite," SIAM J. Sci. Comput., vol. 18, pp. 1-22, 1997.

[305] G. Gamrath, T. Fischer, T. Gally, A. M. Gleixner, G. Hendel, T. Koch, et al., "The scip optimization suite 3.2," Zuse Institute Berlin, Berlin, Germany, 2016.

[306] A. Burnham, J. Han, C. E. Clark, M. Wang, J. B. Dunn, and I. Palou-Rivera, "Life-Cycle Greenhouse Gas Emissions of Shale Gas, Natural Gas, Coal, and Petroleum," Environ. Sci. Technol., vol. 46, pp. 619-627, 2012.

[307] B. H. Gebreslassie, M. Slivinsky, B. Wang, and F. You, "Life cycle optimization for sustainable design and operations of hydrocarbon biorefinery via fast pyrolysis, hydrotreating and hydrocracking," Comput. Chem. Eng., vol. 50, pp. 71-91, 2013.

[308] S. M. Sadrameli, "Thermal/catalytic cracking of hydrocarbons for the production of olefins: A state-of-the-art review I: Thermal cracking review," Fuel, vol. 140, pp. 102-115, 2015.

[309] S. Chin, S. Radzi, I. Maharon, and M. Shafawi, "Kinetic model and simulation analysis for propane dehydrogenation in an industrial moving bed reactor," World Academy of Science, Engineering and Technology, vol. 52, pp. 183-189, 2011.

[310] Y. Chen, T. A. Adams, and P. I. Barton, "Optimal Design and Operation of Static Energy Polygeneration Systems," Ind. Eng. Chem. Res., vol. 50, pp. 5099-5113, 2011.

[311] L. Chen and E. Miller-Hooks, "Resilience: An Indicator of Recovery Capability in Intermodal Freight Transport," Transport. Sci., vol. 46, pp. 109-123, 2012.

[312] K. H. Orwin and D. A. Wardle, "New indices for quantifying the resistance and resilience of soil biota to exogenous disturbances," Soil Biol. Biochem., vol. 36, pp. 1907-1912, 2004.

[313] B. M. Ayyub, "Systems Resilience for Multihazard Environments: Definition, Metrics, and Valuation for Decision Making," Risk Anal., vol. 34, pp. 340-355, 2014.

[314] D. Henry and J. E. Ramirez-Marquez, "Generic metrics and quantitative approaches for system resilience as a function of time," Reliab. Eng. Syst. Safe., vol. 99, pp. 114-122, 2012.

[315] S. E. Chang and N. Nojima, "Measuring post-disaster transportation system performance: the 1995 Kobe earthquake in comparative perspective," Transport. Res. Part A Policy Pract., vol. 35, pp. 475-494, 2001. 
[316] T. Hashimoto, J. R. Stedinger, and D. P. Loucks, "Reliability, resiliency, and vulnerability criteria for water resource system performance evaluation," Water Resour. Res., vol. 18, pp. 14-20, 1982.

[317] S. Enjalbert, F. Vanderhaegen, M. Pichon, K. A. Ouedraogo, and P. Millot, "Assessment of Transportation System Resilience," in Human Modelling in Assisted Transportation: Models, Tools and Risk Methods, P. C. Cacciabue, M. Hjälmdahl, A. Luedtke, and C. Riccioli, Eds., ed Milano: Springer Milan, 2011, pp. 335-341.

[318] E. D. Vugrin, D. E. Warren, and M. A. Ehlen, "A resilience assessment framework for infrastructure and economic systems: Quantitative and qualitative resilience analysis of petrochemical supply chains to a hurricane," Process Saf. Prog., vol. 30, pp. 280-290, 2011.

[319] M. Ouyang and L. Duenas-Osorio, "Time-dependent resilience assessment and improvement of urban infrastructure systems," Chaos, vol. 22, 2012.

[320] S. Ma, B. Chen, and Z. Wang, "Resilience Enhancement Strategy for Distribution Systems under Extreme Weather Events," IEEE Trans. Smart Grid, vol. PP, pp. 1-10, 2016.

[321] "Department of Defense Risk, Issue, and Opportunity Management Guide for Defense Acquisition Programs," Office of the Deputy Assistant Secretary of Defense for Systems Engineering, Washington, D.C., U.S., 2017.

[322] C. Ning and F. You, "A data-driven multistage adaptive robust optimization framework for planning and scheduling under uncertainty," AIChE J., vol. 63, pp. 4343-4369, 2017.

[323] C. Ning and F. You, "Data-driven adaptive nested robust optimization: General modeling framework and efficient computational algorithm for decision making under uncertainty," AIChE J., vol. 63, pp. 3790-3817, 2017. 